\title{
Statistical Evaluation of Randomly Acquired Characteristics on Outsoles with Implications Regarding Chance Co-Occurrence and Spatial Randomness
}

\author{
Nicole Richetelli \\ West Virginia University, nrichete@mix.wvu.edu
}

Follow this and additional works at: https://researchrepository.wvu.edu/etd

Part of the Forensic Science and Technology Commons

\author{
Recommended Citation \\ Richetelli, Nicole, "Statistical Evaluation of Randomly Acquired Characteristics on Outsoles with \\ Implications Regarding Chance Co-Occurrence and Spatial Randomness" (2020). Graduate Theses, \\ Dissertations, and Problem Reports. 7742. \\ https://researchrepository.wvu.edu/etd/7742
}

This Dissertation is protected by copyright and/or related rights. It has been brought to you by the The Research Repository @ WVU with permission from the rights-holder(s). You are free to use this Dissertation in any way that is permitted by the copyright and related rights legislation that applies to your use. For other uses you must obtain permission from the rights-holder(s) directly, unless additional rights are indicated by a Creative Commons license in the record and/ or on the work itself. This Dissertation has been accepted for inclusion in WVU Graduate Theses, Dissertations, and Problem Reports collection by an authorized administrator of The Research Repository @ WVU. For more information, please contact researchrepository@mail.wvu.edu. 
Statistical Evaluation of Randomly Acquired Characteristics on Outsoles with Implications Regarding Chance Co-Occurrence and Spatial Randomness

Nicole Richetelli

Follow this and additional works at: https://researchrepository.wvu.edu/etd

Part of the Forensic Science and Technology Commons 


\title{
Statistical Evaluation of Randomly Acquired Characteristics on Outsoles with Implications Regarding Chance Co-Occurrence and Spatial Randomness
}

\author{
Nicole Richetelli, M.S. \\ Dissertation submitted \\ to the Eberly College of Arts and Sciences \\ at West Virginia University \\ in partial fulfillment of the requirements for the degree of \\ Doctor of Philosophy in \\ Forensic Science \\ Jacqueline Speir, Ph.D., Chair \\ Tatiana Trejos, Ph.D. \\ Glen Jackson, Ph.D. \\ Casey Jelsema, Ph.D. \\ Department of Forensic \& Investigative Science \\ Morgantown, West Virginia \\ 2020
}

Keywords: Forensic, footwear, randomly acquired characteristics, probability, statistical, chance co-occurrence, spatial randomness

Copyright 2020 Nicole Richetelli, M.S. 


\title{
ABSTRACT \\ Statistical Evaluation of Randomly Acquired Characteristics on Outsoles with Implications Regarding Chance Co-Occurrence and Spatial Randomness
}

\author{
Nicole Richetelli, M.S.
}

Footwear evidence holds tremendous forensic value, owing to its ability to formulate linkages between victims, suspects and scenes. Naturally, the strength of these linkages is a function of the perceived clarity, quality and rarity of class, subclass and randomly acquired characteristics (RACs), which are the fundamental outsole features used to formulate source associations. In order to reach a conclusion when performing a footwear comparison, forensic examiners must assign value to the observed similarities and differences that exist between questioned crime scene and test impressions. Embedded within this process is an evaluation of the random association between unrelated shoes as a function of both class and acquired wear characteristics. To date, weight of evidence within this space has been largely informed by the training and subjective casework experience accumulated by an examiner over the life of his or her career. In pursuit of supporting the foundational validity of this comparison process, this research sought to quantify the chance association of RACs on unrelated shoes and the spatial distribution of these features on outsoles, with the long-term goal of aiding weight of evidence assessments in forensic footwear examinations.

Using a large-scale database of 1,300 unrelated outsoles, the position and shape of 72,306 RACs was investigated. Features with consistent position and shape-classification were pairwise compared and sorted using a numerical estimate of similarity. Based on this assessment, more than 91,000 of the most quantitatively similar features were visually evaluated in order to model the relationship between numerical similarity and visual indistinguishability. Using this model, more than 1 million additional feature comparisons were evaluated in order to predict the potential for visual confusion. Subsequently, empirical and modeled probabilities of indistinguishability were combined with the chance for positional overlap to yield location- 
and shape-specific estimates of chance association. The results indicated that RACs exhibit high discriminating potential, with median chance associations ranging from 1 in 541,276 to 1 in 18,031,824, depending upon shape. However, additional inspection revealed that chance association was not constant across an outsole. Given this secondary observation, the spatial distribution of RACs on outsoles was further investigated. In order to conduct this analysis, a set of over 1.7 million null and 1.9 million alternative contact-modified synthetic distributions were simulated for comparison against the collected empirical data. Results indicated that Poisson null distributions (both synthetic and modeled) well-describe the frequency of RACs across approximately $64 \%$ of an outsole. Moreover, the regions not well represented by a random distribution were highly localized to three general areas (ball of the toe, arch, and edge of the heel). Based upon this observation, it was purported that an important theoretical or practical factor was additionally required to improve prediction in these locations.

Therefore, spatial regression modeling was utilized in order to assess the impact of spatial effects on RAC distributions. Under optimal conditions, $87 \%$ of location-specific RAC counts were well predicted using contact area and incorporating neighboring cells data for contact and accidentals (a $67 \%$ performance increase over non-spatial predictions). Based upon a visual inspection of the remaining $13 \%$ of cells with persisting residual correlation, it was hypothesized that wear (the intersection of contact and use) may further improve model predictions and a proof of concept study was conducted to evaluate this theory. After incorporation of contact-localized wear as a predictor in the spatial models, nearly $96 \%$ of the outsole was well described. Considered collectively, the results from this work indicate that RACs are sufficiently rare, owing to variability in position, shape, and geometry, to differentiate shoes, as evidenced by the low probabilities of stochastic chance association between unrelated features. Furthermore, the majority of feature frequencies across the outsole can be adequately described by tread contact alone, irrespective of position. However, positional considerations for evidentiary value must be incorporated for features occurring in three specific areas including the ball of the toe, the arch, and the edge of the heel. Ulti- 
mately, the results from this study provide fundamental knowledge about the practical and theoretical/statistical factors that underpin the spatial distribution and subsequent weight of evidence of RACs for footwear evidence. 


\section{Acknowledgments}

Portions of this project were supported by Award No. 2013-DN-BX-K043 and Award No. 2018-MU-MU-0033, awarded by the National Institute of Justice, Office of Justice Programs, U.S. Department of Justice. The opinions, findings, conclusions and recommendations expressed in this presentation are those of the authors and do not necessarily reflect those of the Department of Justice.

Portions of this project were completed using supercomputing systems (Spruce Knob and Thorny Flat), which are funded in part by the National Science Foundation EPSCoR Research Infrastructure Improvement Cooperative Agreement \#1003907, National Science Foun-

dation Major Research Instrumentation Program (MRI) Award \#1726534, the state of West Virginia (WVEPSCoR via the Higher Education Policy Commission) and West Virginia University (WVU). 
This work is dedicated to my grandpa, Roger Moore, who inspired me to pursue my PhD and offered unending encouragement and support. 


\section{Contents}

$\begin{array}{llr}1 & \text { Introduction } & 1\end{array}$

\begin{tabular}{|ll|}
\hline 2 & Quantification of RAC Co-Occurrence and Confusion
\end{tabular}

\begin{tabular}{|lll}
\hline 3 & Evaluation of Spatial Randomness & 48
\end{tabular}

4 Spatial Modeling of RACs as a Function of Outsole Factors: Part I 99

5 Spatial Modeling of RACs as a Function of Outsole Factors: Part II $\quad 127$

$\begin{array}{llr}6 & \text { Final Remarks } & 170\end{array}$

6.1 Observations and Future Directions $\ldots \ldots \ldots$. . . . . . . . . . . 170

6.1 .1 Chance Association of RACs . . . . . . . . . . . . . . . 170

$6.1 .2 \quad$ Spatial Distribution of RACs $\ldots \ldots \ldots \ldots \ldots \ldots$

6.2 Addressing Remaining Research Needs $\ldots \ldots \ldots$. . . . . . . . . 174 


\section{Introduction}

Within the past decade, forensic science has come under immense scrutiny with several major reports highlighting issues in the practice, reporting, and utilization of established methods for various subject areas [1,2]. Possibly the most criticized of all fields are those based in pattern evidence, including footwear, likely because these approaches typically utilize the expert as the instrument of analysis, thereby making analyses inherently more subjective [1,2]. While forensic pattern experts are innately able to identify minute details and form meaningful linkages - owing to extensive education, training, and casework — it is important that they are using appropriate features for their comparisons. More specifically, these characteristics must hold high discriminating power and be useful for differentiating between similar items of the population, thus aiding in reaching source attribution. Furthermore, the proper evaluation and assignment of probative value by these experts is of utmost importance in order to ensure that evidence is used properly in the justice system and undue weight is not ascribed that could ultimately cause a criminal to walk free or an innocent person to be found guilty.

Although it is clear that proper assignment of evidential value is essential to the practice and utilization of forensic science, additional research must be conducted to understand the factors underpinning it. When assessing weight of evidence with regard to footwear impressions, several factors must be considered and explored, including (i.) the quality and totality of the submitted questioned impressions as a function of media, substrate, and deposition factors, (ii.) the features available for comparison (class, subclass, randomly acquired), (iii.) the observed agreement or discrepancies between the features in the crime scene and known impressions, (iv.) the discriminating potential of the available features, (v.) the spatial relationship, or lack thereof, between features, and (vi.) the perceived rarity of 
the associated features as a function of examiner accumulated experience. Of all the factors that impact the evidental value, two are the most related to foundational validity of footwear evidence because they involve the features that are actually used to reach source attributions. First, it is necessary to understand the chance that two features on unrelated shoes (known non-matches) could be confused as originating from the same outsole, akin to a random match probability in DNA evidence analysis. Second, after establishing the discriminating potential of an observed association between accidentals, the focus then becomes the spatial factors that impact this magnitude. This question requires research to explore the spatial distribution of randomly acquired characteristics (RACs) and determine whether they are truly random, or if there are underlying spatial patterns that must be accounted for in the evaluation of feature weights. Therefore, the proposed research seeks to investigate these two factors that heavily impact the accurate assignment of weight of evidence in an initial effort to provide empirical support to the analysis of forensic footwear evidence.

When conducting an analysis between crime scene and known impressions, conclusions regarding identity are reached by considering the class, subclass, and randomly acquired characteristics available for comparison [3]. Accordingly, the perceived value of the features detected and utilized in a footwear comparison is a function of the combined types, clarities, and rarities of the features themselves. As such, forensic footwear evidence examination is conducted using a highly methodological and sequential process to compare a crime scene impression to a submitted known or suspect shoe and identify features of interest for either associating or differentiating these two items [4,5]. Initially, an expert assesses the class characteristics present on both items, including outsole design, overall dimensions of the outsole, and size or spacing of individual tread elements, followed by an evaluation of subclass characteristics and wear features. Several empirical studies have been conducted to assess the utility of class and wear features, both alone and in combination [6 11]. The collective results from these works indicate that while class and wear features hold tremendous value for narrowing a suspect pool down to a minute fraction of the original population, they cannot be used alone to reach a source attribution because the discriminating power is not sufficiently strong (though disagreement of class/wear can certainly be used as a means for 
exclusion).

It follows, then, that acquired features hold the key to reaching source attribution. Given the highly variable and dynamic nature in which accidentals are created as a function of wear (which is accordingly dependent on the outsole material, the wearer's height, weight, and gait, the surface and terrain in contact with the outsole, and the activity being performed, just to name a few), it stands to reason that these features would be highly discriminating as they are expected to vary between shoes. In fact, several theoretical and empirical studies have supported this assertion $[12 \sqrt{18}]$ showing that even when RACs appeared in the same location (irrespective of the class characteristics of the outsole) and of relatively the same shape, their sizes, orientations, and/or perimeter morphologies were useful for distinguishing between features. Although the results from each of the previous studies reinforce the longheld belief that acquired features are suitable for informing source attributions, wherein outsoles can be differentiated and associated based on these characteristics due to their highly variable nature, this current body of work suffers from several limitations. First, the theoretical studies utilize several untested assumptions in their computations for chance of RAC co-occurrence, such as independence in feature location and/or attribute (e.g., shape, orientation), and inherently lack the ability to visually compare features for their potential of confusion [12, 14]. In addition, while the empirical studies do conduct visual comparisons of RACs in addition to considerations for location, general shape, and/or orientation, they each use relatively small databases sizes, likely owing to the immense manual task of comparing large numbers of accidentals [13, 15, 17]. In an effort to mitigate these limitations and further characterize the discriminating potential of accidentals, the proposed research will conduct a large-scale assessment of the chance association of RACs on unrelated outsoles by combining the chance of co-occurrence in position and shape with the chance of confusion as a function of visual assessments, which is detailed further in a stand-alone paper constituting Chapter 2 of this document.

Once the chance of random association for a single RAC pair is quantified, it then becomes imperative to understand how weight of evidence varies across an outsole. In order to conclude an identification of source between a crime scene impression and known footwear, 
agreement of one or more RACs are required. Despite the large body of work regarding the usefulness of RACs as features for reaching source attributions, considerably less work has been conducted to inform the next step in an examiner's comparison and evaluation process. Once an examiner observes accidentals in common between a known and a questioned impression, he or she must assign a weight to the evidence in totality in order to reach a final conclusion, which is a function of the perceived rarity of the features themselves and/or in combination [3]. Therefore, in order to properly assess the value of these features, an examiner must first understand the weight of each feature as a function of its location. For example, if RACs are more likely to accrue in the ball of the toe, then an observed similarity in this region is less informative than one in an area where chance associations are lower, such as the arch. Given the potential impact on assessment of associations for reaching conclusions, an examination of spatial randomness of accidentals is necessary to foster this understanding. To date, only three studies have commented on the distribution of features on outsoles $[18$ 20]. The conclusions from these previous attempts at describing the arrangement of features on outsoles are in disagreement, albeit all three utilized vastly different approaches. However, given the limited body of work in this area, and the conflicting results for the few publications that do evaluate RAC distribution, there remains ambiguity in how to assign weight of evidence to concordances between features. Thus, the proposed research seeks to statistically evaluate the spatial distribution of accidentals as a function of relevant outsole factors to inform the assessment of evidential value for features utilized in footwear comparisons, which is discussed in more detail in Chapters 3, 4, and 5 of this document. 


\section{Quantification of RAC \\ Co-Occurrence and Confusion}




\section{Empirically Observed and Predicted Estimates of Chance Association: Estimating the Chance Association of Randomly Acquired Characteristics in Footwear Comparisons}

Keywords: Chance Co-occurrence, Indistinguishability, Randomly Acquired Characteristics, Bernoulli distribution, Logistic Regression

\section{Introduction}

The power associated with demonstrating a linkage between a shoe and the impression it is alleged to have produced at the scene of a crime is directly related to the quality and quantity of manufactured class (design and dimension), acquired wear, and randomly acquired characteristics (RACs). When individualizing characteristics are present, their relative position, orientation, size and shape are examined and compared with known exemplars [1] in an effort to establish the strength of the suspected linkage. However, the degree to which a feature, or a collection of features, might repeat by chance alone is less well-documented. At times, the question posed is the chance of randomly selecting two unrelated outsoles from a population and finding that they both possess forensically indistinguishable features. Alternatively, the casework question is typically concerned with the random match probability, or the probability that an unrelated randomly selected outsole would be considered indistinguishable from the questioned evidence. Regardless of the question asked, the likelihood of encountering a close non-match within the pattern sciences is most often formalized by an examiner's accumulated expertise, wherein an analyst with years of experience develops internalized knowledge as to the likelihood that a feature (or set of features) might reproduce by chance alone between two known non-matches (KNMs). Despite reasonable validity associated with using accumulated expertise to inform evidence interpretation, there is still a need for external theoretical models and empirical investigations to support these inferences. Unfortunately, providing objective data in support of these assertions is not easily achieved, and all attempts are invariably bounded by one or more constraints, including theoretical 
model assumptions and/or empirical database size limitations (wherein the latter obstacle should not be underestimated since it is extremely difficult and time-consuming to obtain a sufficiently large sample size for reliable estimation).

Although several former studies have attempted to shed light on variants of the chance estimate question [2-19], it has been difficult to relate theoretical models with empirical observations. Sometimes the discrepancies may be the result of asking different questions, but equally likely, differences can arise when models are bounded by untested or partially tested assumptions (such as independence in RAC location, RAC attribute, or both), and empirical datasets that are too small to allow for statistical analyses of sufficient power. To illustrate some of the similarities and differences in various approaches, the following (although not necessarily an exhaustive review) attempts to highlight and succinctly summarize the current state of research on this topic as it relates to forensic footwear analysis.

\section{Theoretical Models of Chance Association}

In perhaps one of the earliest published approaches to this problem, Fawcett [13] modeled the chance agreement of accidental characteristics between test and crime scene impressions using the binomial coefficient, as illustrated in Eq. (1) where ${ }_{n} C_{k}$ describes the number of ways of selecting $k$ unordered outcomes from $n$ possibilities, which inherently assumes that each observation is independent and of equal probability.

$$
{ }_{n} C_{k}=\left(\begin{array}{l}
n \\
k
\end{array}\right)=\frac{n !}{(n-k) !(k) !}
$$

Using this expression as the basis for the model, Fawcett [13] estimated the average chance agreement of $p$ details between impressions, accounting for variation in resolution, as well as differences (real or aberrant) between the scene and exemplar prints, resulting in a report of how often one can expect agreement of $p$ details 'by chance' for a known non-match comparison involving $s$ possible discrete locations, when $x$ features are detected on the questioned impression, and $z$ are detected on the test impression, according to Eq. (2) (summing from $p=p$ to $p=x$ (or $z$ ), which can often be simplified to $p=p$ exclusively).

$$
\text { Chance agreement of } 1 \text { in }=\frac{{ }_{s} C_{x}}{\sum_{p}\left({ }_{z} C_{p}\right)\left({ }_{s-z} C_{x-p}\right)}
$$


To illustrate, Fawcett [13] presents the following example, that includes an exemplar impression with $z=2$ nicks, a crime scene impression with $p=2$ RACs in agreement between the questioned and test impression, but $x=10$ possible voids. If the crime scene impression is $2.5 \mathrm{~cm}^{2}$ and using the size of the mated-features as a basis for location/resolution, then the questioned impression can be divided into a total of $s=86$ similarly sized regions, resulting in an average chance agreement of approximately 1 in 80 as per the following computation:

$$
\text { Chance agreement of } 1 \text { in }=\frac{{ }_{86} C_{10}}{\sum_{p=2}\left({ }_{2} C_{p}\right)\left({ }_{84} C_{10-p}\right)}=81.2
$$

In a second theoretical approach, Stone [9] purported to compute the theoretical probability of the random duplication of accidental features with increasing degrees of complexity (points, lines, curves, etc.), while assuming a questioned impression of full resolution, free of both pseudo-accidentals and mismatched features, and the complete independence between RAC location and attribute. In this model, Stone [9] assumed a hypothetical flat-soled men's size 8.5 shoe with a working area of $16,000 \mathrm{~mm}^{2}$ and a $1 \mathrm{~mm}^{2}$ resolvable limit. Using these model parameters and the aforementioned assumptions, the computed probability of random duplication of a single point feature (defined as a defect with 'no discernible length or width') was reported to be 1 in 16,000 .

Extending this computation to account for more complexity, including the geometry of a feature of interest, larger defects (such as lines and curves) that possess a greater number of feature attributes were estimated through multiplication of RAC location and attributes (such as a line's length and orientation), as illustrated in Eq. (3), again assuming independence in RAC position and attribute.

$$
\text { Chance occurrence of } 1 \text { in }=\frac{1}{p(\text { shape }) \times p(\text { orientation }) \times p(\text { position })}
$$

To illustrate, if the length and orientation of a line are binned and assumed to be equally probable anywhere on the outsole, then for lines divided into one of three possible lengths (short, medium and long), and orientations divided into one of eight different possibilities between $0^{\circ}$ and $360^{\circ}$, then the random duplication of a single line is computed as 1 in 384,000 as shown by Stone [9]: 


$$
\text { Chance occurrence of } 1 \text { in }=\frac{1}{\frac{1}{3} \times \frac{1}{8} \times \frac{1}{16,000}}=\frac{1}{2.60417 \mathrm{e}-6} \approx 384,000
$$

\section{Using Empirical Data to Inform a Model}

Although not necessarily claimed by Yekutieli et al. [17] as the inspiration for the work conducted in a more recent study, this group's technical report describing research funded by the National Institute of Justice (NIJ) in the area of footwear and statistical modeling is an interesting use of empirical data to inform some of the unknown and assumed parameters in Stone's [9] theoretical model. Yekutieli et al.'s [17] group created two large empirical databases (believed to constitute the first of their kind in terms of magnitude and characterization). The first consisted of 8,900 accidentals collected from 300 test impressions with known information regarding both location and feature contour, while the second consisted of 20,000 accidentals from 600 test impressions, but limited in description to location only [17]. Assuming independence, Yekutieli et al. [17] modeled the probability of a RAC as a function of location, orientation, and shape, analogous to Eq. (3) as offered by Stone [9], but modified (i.e., informed) by empirical observations. First, the group assumed a shoe with an area of $30,000 \mathrm{~mm}^{2}$ and a resolvable limit of $25 \mathrm{~mm}^{2}$ (which differs from Stone's [9] assumption of $16,000 \mathrm{~mm}^{2}$ and $1 \mathrm{~mm}^{2}$, respectively). This was used to define $p$ (selection) according to $1 /[30,000 / 25]=1 / 1,200$ or the probability of selecting a location at random. The probability of finding a RAC at this location was then found to be $30 \times p$ (selection) $=1 / 40$ based on the fact that an average of 30 RACs were found per shoe (assuming that RACs are uniformly distributed over outsoles, which the authors indicate cursory testing confirmed). Next, the group reports that orientations are uniformly distributed (except for very long shapes), and that the shape of the feature dictates its orientation error (e.g., a circularly shaped feature has very little real orientation). Given this, $p$ (orientation) was assumed equal to [orientation error $\left./ 180^{\circ}\right]$. Finally, $p$ (shape) was empirically estimated by 
first creating known match (KM) and known non-match (KNM) distributions, and then determining a threshold for describing shapes as 'similar enough' (to be indistinguishable) based upon a Modified Hausdorff distance. The similarity metric threshold to define 'similar enough' was determined based on 82,000 known match and 100,000 known non-match comparisons, making $p$ (shape) equal to [\# RACs similar enough/total] [17]. As an example calculation, the group reports the following for a shape with orientation error of $5^{\circ}$ (e.g., $p($ orientation $)=5 / 180)$, that is similar to 50 out of 10,000 shapes in the database (e.g., $p($ shape $)=50 / 10,000)$, and $p($ position $)=1 / 40$ (as formerly described $),$ leading to a random chance of 1 in 288,000 as illustrated by Yekutieli et al. [17]:

$$
\begin{aligned}
\text { Chance occurrence of } 1 \text { in } & =\frac{1}{p(\text { shape }) \times p(\text { orientation }) \times p(\text { position })} \\
& =\frac{1}{\frac{50}{10,000} \times \frac{5}{180} \times \frac{1}{40}}=\frac{1}{3.47222 \mathrm{e}-6} \approx 288,000
\end{aligned}
$$

Note the similarity with Stone's [9] report (1 in 384,000), but arrived at using similarity metrics, orientation and orientation error, and accounting for the fact that of the number of ways a location can be selected, only a fraction are expected to be occupied by RACs.

\section{Empirical Observations of Chance Association}

In contrast with the theoretical models presented by Fawcett [13] and Stone [9], and a theoretical model informed using empirical data as presented by Yekutieli et al. [17], Cassidy [2] attempted to answer the question of chance reproduction of individual characteristics based on empirical studies. Using groups of police recruits engaged in activities presumed to promote the chance reproduction of accidental characteristics, Cassidy [2] concluded that there was a 1 in 6 chance of finding 10 'minute' characteristics and a 1 in 20 chance of finding 3 'moderate-sized' characteristics possessing coincidental similarity in position for the heel 
of compared shoes. However, Cassidy [2] acknowledged that similarities based on mold may very well be included in these figures, and an extensive discussion of the similarity in feature quality was not pursued. When only a single feature is considered, Cassidy [2] found that a 'moderate-sized' characteristic has a 1 in 38 (for 38 compared shoes) to a 1 in 60 (for 60 compared shoes) chance of possessing coincidental similarity in position with known nonmatch heels [2] (note that it is unclear if it is 'coincidental' that the chance is equal to the total number of compared shoes in each study, or if this was a product of the study's design).

Of course, the heel of a shoe is only a fraction of the size of the shoe modeled by Stone [9], but even assuming that the heel is a fourth of the total area of the outsole, the empirical results presented by Cassidy [2] suggest a higher probability of chance similarity in position (when shape is ignored, or 1 in 16,000), and closer to reports of $p$ (position) $=1 / 40$ by Yekutieli et al. [17]. Although one could argue that this difference is a function of the shared activities performed by the police recruits (e.g., activities believed to favor the duplication of shared features), this is only speculative since an empirical baseline does not exist that can describe the chance duplication of random characteristics for individuals and activities that are unrelated or random. Perhaps equally likely, it could be that Cassidy [2] was reporting random match probability, while Stone [9] was interested in the chance of selecting two shoes at random and finding a positional match; naturally, the way the question is posed has a marked impact on the reported probability.

In addition to Cassidy's [2] work, Wilson [5] also executed an empirical study to determine if RACs present on outsoles could be used to differentiate shoes when the chance for co-occurrence was presumed to be enhanced based on the nature of the study (which consisted of 39 pairs of size 12 men's shoes, all of the same make and model, with a reported average wear of 341 miles — with a minimum of 55 and a maximum of 449 — all worn by the same individual, and with some consistency in track-location given that the 
wearer/runner had fairly prescribed running-routes) [5]. After dividing the outsole into subregions (coincidentally, 39 subregions were defined, as a function of the outsole pattern for the Adidas ${ }^{\circledR}$ Supernova Classic shoe used in this study), the number, location and geometry of the features were cross-compared, and it was determined that all shoes could be pairwise differentiated [5].

In an analogous study, Marvin [16] examined 34 pairs of New Balance ${ }^{\circledR}$ model 878 outsoles (again, all worn by the same person, running approximately the same distance, on approximately the same surfaces, thereby reasonably maximizing the possibility of chance association). Using this dataset, several questions were asked, of which two directly relate to chance. First, all lefts and rights could be differentiated based on a visual assessment of 'wear' (which required 2,244 paired comparisons or $n(n-1) / 2=34(33) / 2=561$ lefts and 561 rights, performed in duplicate by two certified footwear examiners) [16]. Second, following the extraction and localization (to $5 \mathrm{~mm} \times 5 \mathrm{~mm}$ cells) of more than 13,000 RACs, a visual assessment of chance association was conducted based on RACs in the most populated cell (requiring 2,850 paired-comparisons). Again, results indicated that co-occurring RACs were differentiable based on shape/geometry [16] (although additional work is needed to determine if this conclusion persists when comparing all other mated-cells).

Similar results are replicated in two additional studies, but based on smaller datasets. Adair et al. [14] obtained 12 pairs of Altitude II hiking boots, and a single pair was worn by one of six participants during ascent of Mount Bierstadt in Colorado. A second pair was worn during descent, and when all pairs were examined, no correspondences were found. Similarly, Hamburg and Banks [15] evaluated four pairs of shoes (worn by two participants), at 35 specific usage intervals (impressions were collected every $\sim 4,000$ steps to a maximum of 138,000$)$, and again, it was found that no acquired marks repeated.

In a comparable effort conducted by the author's research group, Khalil [18] examined 38 

not explicitly investigated as part of this work).

\section{Probability Density Function of Known Match \& Known Non-Match Correlation Coefficients}

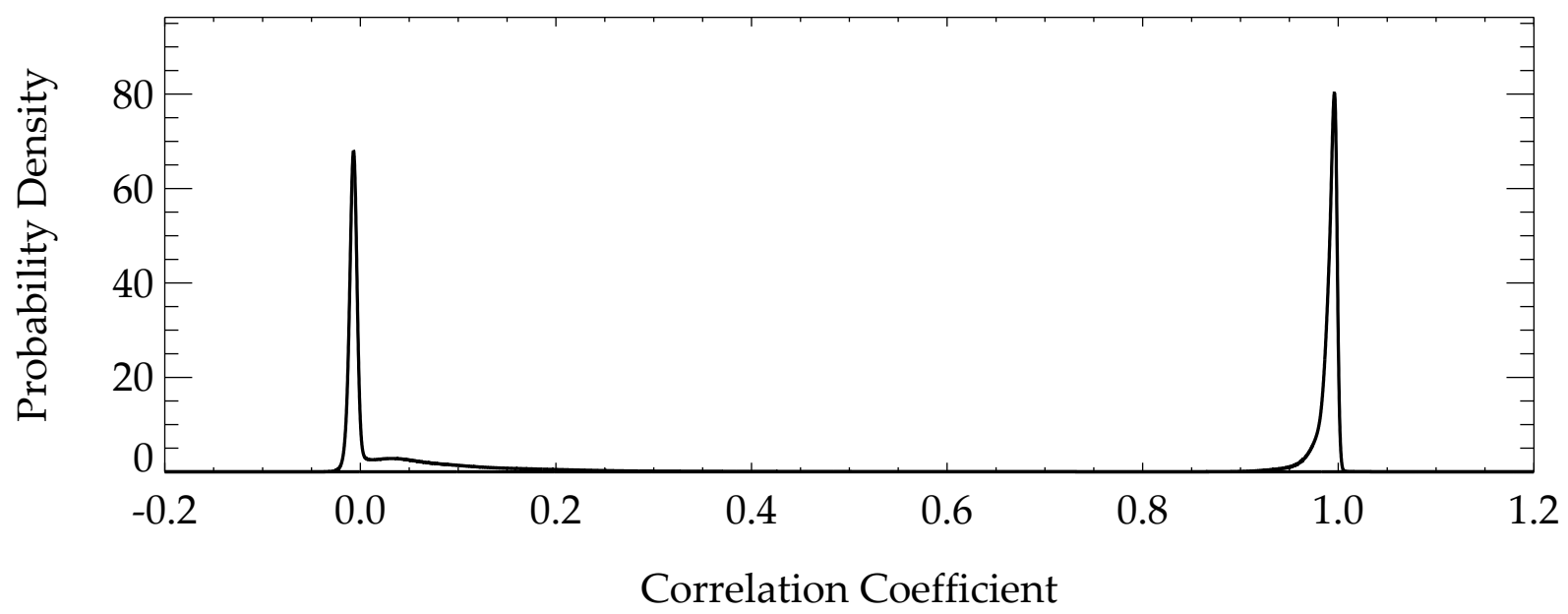

Figure 1: Probability density plot of known match correlation scores $(\sim 1)$ and known non-match correlation scores $(\sim 0)$ based on 34,218 pairwise comparisons, reproduced using results from [18] .

170

outsoles of the same make and model worn by 19 participants, collected every 20,000 steps (up to 220,000 in total) over the course of seven-months of use. All pairwise comparisons $(34,218)$ were evaluated using the numerical metric of correlation and the results indicated high discrimination potential, effectively separating known match and known non-match scores as illustrated in Fig. 1 (however, the potential for individual features to co-occur was

Correlation Coefficient

\section{Lessons Learned}

At this point it is useful to summarize the published work on the topic of chance association of RACs from unrelated sources. Although each research effort differed substantially, Table 1 attempts to generalize the review into a high-level message that can be used to formulate two main points. First, all theoretical models assume independence in RAC location and attribute, which has not yet been fully vetted as a valid assumption. In fact, 
empirical results regarding this matter are either anecdotally or statistically suggestive of dependence. For example, Davis and DeHaan [12] evaluated 650 pairs of men's shoes and reported that defects were rarely encountered in the rear and central portions of the heel. This observation was drawn after analyzing the frequency of features located in 16 different regions on the heel of outsoles (but it is unclear if the frequency information was based on 317 examinations, or all 650 shoes). The authors go on to attribute this observation to high wear and continual erosion in these regions of the heel, and as a corollary, Davis and DeHaan [12] suggest that a higher significance should be assigned to any accidental that is found on the mid- to rear-section of the heel. Unfortunately, the published results do not take this observation to completion, which would require a statistical analysis to determine if the observed frequency of detected features in these regions are, indeed, statistically lower than that observed in other regions.

More recently, Damary et al. [19] subjected 3,500 RACs from 380 laboratory impressions to several chi-square tests of independence, reporting that RAC shape and orientation, RAC shape and location, and RAC orientation and location are all dependent (for a normalized outsole divided into 14 pre-selected regions for comparison). Given such preliminary statistical and anecdotal evidence for dependence, if this observation is found to persist in additional studies, then chance estimates using theoretical models based on independence may require modification moving forward.

Second, all empirical/visual comparisons report essentially no chance associations when RAC geometry is included in the comparison (i.e., the results reported by Cassidy [2] are restricted to estimates for co-occurrence in position, without regard for RAC geometry and similarity). However, the sample size for each study reporting zero associations is limited to, at most, 39 pairs of shoes. Thus, the results must be re-evaluated using a larger dataset since inference suggests that with a large enough sample size, chance association must be greater 
Table 1: Summary of estimates of chance association of acquired characteristics from both modeled and observed datasets. Note that the work of Wilson [5] and Khalil [18] are not included in the table since these studies did not specifically discuss the association of RACs in known non-matches, and instead, report zero associations between known non-matches when evaluating full RAC patterns or RAC maps (visually by Wilson [5] and mathematically by Khalil [18]).

\begin{tabular}{|c|c|c|c|c|}
\hline Author & Approach & Example Result & $\begin{array}{c}\text { Method/ } \\
\text { Assumption }\end{array}$ & Description \\
\hline Fawcett [13] & $\begin{array}{c}\text { Binomial } \\
\text { Coefficient }\end{array}$ & $\begin{array}{c}\text { Two Features } \\
1: 80\end{array}$ & $\begin{array}{l}\text { Theoretical/ } \\
\text { Independence }\end{array}$ & $\begin{array}{c}z=2, p=2 \\
x=10, s=86\end{array}$ \\
\hline Stone $[9]$ & $\begin{array}{c}\text { Multiplication } \\
\text { Rule }\end{array}$ & $\begin{array}{l}\text { Line } \\
1: 384,000\end{array}$ & $\begin{array}{l}\text { Theoretical/ } \\
\text { Independence }\end{array}$ & $\begin{array}{c}3 \text { line lengths, } \\
8 \text { possible orientations }\end{array}$ \\
\hline Yekutieli et al. [17] & $\begin{array}{c}\text { Multiplication } \\
\text { Rule }\end{array}$ & $\begin{array}{l}\text { Line } \\
1: 288,000\end{array}$ & $\begin{array}{l}\text { Theoretical+Empirical/ } \\
\text { Independence }\end{array}$ & $\begin{array}{c}\text { location, } \\
\text { orientation, similarity }\end{array}$ \\
\hline Cassidy [2] & $\begin{array}{c}\text { Visual } \\
\text { Comparison }\end{array}$ & $\begin{array}{l}\text { Position } \\
1: 38-1: 60\end{array}$ & $\begin{array}{l}\text { Empirical/ } \\
\text { None? }\end{array}$ & $\begin{array}{c}\text { same make, model }(1: 38) \\
\text { four models }(1: 60) ; \\
\text { shared activities }\end{array}$ \\
\hline Marvin [16] & $\begin{array}{c}\text { Visual } \\
\text { Comparison }\end{array}$ & $\begin{array}{c}\text { Most Populated } \\
\text { Cell 0:5,551 }\end{array}$ & $\begin{array}{l}\text { Empirical/ } \\
\text { None? }\end{array}$ & $\begin{array}{l}\text { same make, model } \\
\text { and wearer }\end{array}$ \\
\hline Adair et al. [14] & $\begin{array}{c}\text { Visual } \\
\text { Comparison }\end{array}$ & $0: \mathrm{X} \dagger$ & $\begin{array}{l}\text { Empirical/ } \\
\text { None? }\end{array}$ & $\begin{array}{l}\text { same make, model; } \\
\text { six participants }\end{array}$ \\
\hline Hamburg and Banks [15] & $\begin{array}{c}\text { Visual } \\
\text { Comparison }\end{array}$ & $0: \mathrm{Y}^{\prime} \dagger$ & $\begin{array}{l}\text { Empirical/ } \\
\text { None? }\end{array}$ & $\begin{array}{l}\text { same make, model; } \\
\text { two participants }\end{array}$ \\
\hline
\end{tabular}

$\dagger \mathrm{X}$ not available, but based on 24 outsoles; $\dagger \dagger \mathrm{Y}$ not available, but based on 8 outsoles.

than zero. In other words, RACs do co-occur in position (Cassidy [2] has already observed this, and it is further validated by inference since the physical area of an outsole is limited, and the tread-elements in contact with the ground are further limiting, which consequently limits the surface area on which RACs can develop). In addition, if the geometry of the feature is simple (such as a small, isotropic, circularly-shaped pin-prick) then with a large enough sample size, an observer should find two nondescript RACs that coincide by chance. The remainder of this work considers this question using an empirical database of 72,306 RACs collected from 1,300 outsoles, while a subsequent study aims to estimate random match probability for the same dataset. 


\section{Materials and Methods}

\section{Dataset}

A total of 72,306 RACs were detected and characterized after sampling 1,300 outsoles with extensive variation in class characteristics, such as size, manufacturer, model and degree of wear (for additional details regarding the source and methodology by which the dataset was collected, please refer to the technical report by Speir et al. [20]). Following detection and localization, each RAC was remapped to a standard outsole, corresponding to a men's size 10 Reebok ${ }^{\circledR}$ walking shoe with a surface area of $21,235 \mathrm{~mm}^{2}$. Beginning from the top medial portion of the shoe, the outsole was divided into $5 \mathrm{~mm} \times 5 \mathrm{~mm}$ cells through a rastering process, creating 987 populated cells, of which 835 were complete, and 152 were deemed partial (i.e., straddling the perimeter/edge of the outsole, and with a surface area $\leq 95 \%$ of a complete cell). Note that rastering across a rectangular plane that encompassed the standard outsole created 990 cells of which 860 were complete (with regard to area) and 130 straddled the perimeter, as reported in [20]. However, after increasing the database size to 1,300 (from 1,000 [20]), updating the RAC-localization rule so that any $R A C$ that borders two cells is always placed into the left-hand cell, and updating the definition of a partial cell, which was formerly defined as any cell with an area less than $25 \mathrm{~mm}^{2}$, but is now reported as cells with areas $\leq 95 \%$ of the area of a complete cell, the finalized heatmap contains a total of 987 cells, of which 835 are complete, and 152 have areas less $23.75 \mathrm{~mm}^{2}$.

Post localization to a standard outsole, a frequency heatmap was created, where color references density or the frequency of RACs per cell, as illustrated in Fig. 2. This is also available as an interactive webpage (www.4n6chemometrics.com/database/) wherein the user can view additional information related to the dataset.

With regard to the heatmap and summary statistics illustrated in Fig. 2 (and accessible from the aforementioned URL), the top shaded row in the summary table is static, and 


\begin{tabular}{|c|c|c|c|c|}
\hline & Variable & Linear & Compact & Any Shape \\
\hline All RACs in Database & 28,236 & 21,363 & 22,707 & 72,306 \\
\hline All RACs in Cell & 25 & 17 & 27 & 69 \\
\hline Chance of Finding RAC in Cell & 1 in 2,892 & 1 in 4,253 & 1 in 2,678 & 1 in 1,047 \\
\hline Unique Shoes Contributing to RAC Count & 23 & 17 & 24 & 61 \\
\hline Shoes with Tread Contact in Cell & 925 & 923 & 925 & 927 \\
\hline Chance of RAC Co-Occurrence & 1 in 1,689 & 1 in 3,128 & 1 in 1,548 & 1 in 234 \\
\hline Chance of RAC Indistinguishability & 1 in ? & 1 in ? & 1 in? & NA \\
\hline Visually Evaluated / Total RAC Pairs & $? / ?$ & $? / ?$ & $? / ?$ & NA \\
\hline Similarities \& Likelihood Ratios & Variable & Linear & Compact & Any Shape \\
\hline
\end{tabular}

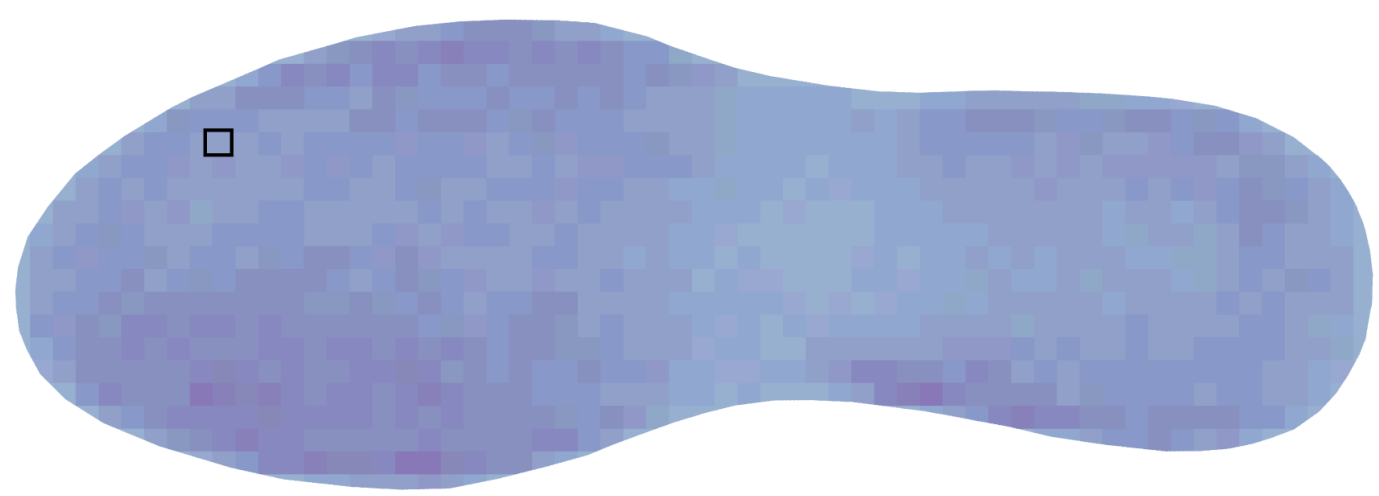

Low Density

High Density

Figure 2: Heatmap and frequency of RACs within $5 \mathrm{~mm} \times 5 \mathrm{~mm}$ cells. Note that the summary table reports the results for the highlighted cell with $\mathrm{x}$ - and $\mathrm{y}$-coordinates $(16,50)$, and rows labeled "Chance of $R A C$ Indistinguishability" and "Visually Evaluated/Total RAC Pairs" are the focus of this paper.

reports the total number of RACs in the database, irrespective of location. Conversely, the remaining rows dynamically update to provide location-specific information upon receiving a cell-selection from the user. For example, the summary table in Fig. 2 reflects a userselection to inspect cell $(16,50)$, and the row labeled "All RACs in Cell" reports the total number of RACs in this specific cell. Similarly, the row labeled "Chance of Finding RAC in Cell" reports the empirical frequency of finding a RAC at the selected location. This is computed from the ratio of $72,306 /$ (\# of RACs in Cell), and truncating the result using 
the floor function in order to be as conservative as possible (e.g., 72,306/69 $=\lfloor 1,047.9\rfloor=$ 1 in 1,047 for all RACs in this cell, regardless of shape categorization). For the remaining columns in the table, the result is repeated, but for RACs categorized as variable, linear or compact in appearance (please see [20] for details regarding former shape categorization labels and methods while details regarding updates to these shape categorization labels are discussed in a supplementary materials section). The row labeled "Unique Shoes Contributing to $R A C$ Count" reports the number of shoes contributing to the "All RACs in Cell" count. In other words, 25 variable-features were found in cell $(16,50)$, contributed by 23 different shoes, meaning one shoe or more contributed at least two variable features to this count. The row labeled "Shoes with Tread Contact in Cell" reports the number of shoes (out of a possible 1,300 ) that had an outsole pattern in contact with the ground at a specific spatial location. This was an important variable to ascertain since few shoes in this dataset had flat and uniform outsoles, and if an outsole does not have tread in contact with the ground at a specific position, then it is unlikely to exhibit a RAC at this location (at least when examining 2-dimensional impressions and barring the acquisition of foreign material into recessed pattern areas).

To determine which shoes had tread in contact with the ground at specific cell locations, each test impression (top row, Fig. 3) was converted to a tread element binary mask (bottom row, Fig. 3) where black illustrates tread elements in contact with the ground, while white corresponds to raised areas. This tread element binary mask was generated through an image processing sequence that included several transformations, including downsampling, median filtering using a $3 \times 3$ window, mode subtraction, thresholding using $-0.5 \times \sigma_{i}$ where $\sigma_{i}$ is the image standard deviation, adaptive histogram equalization, and edge detection using a difference of Gaussian (DOG) with $\sigma_{D O G}$ between 3.0 and 5.0.

Using these tread element binary masks, each of the 1,300 outsoles was queried to deter- 


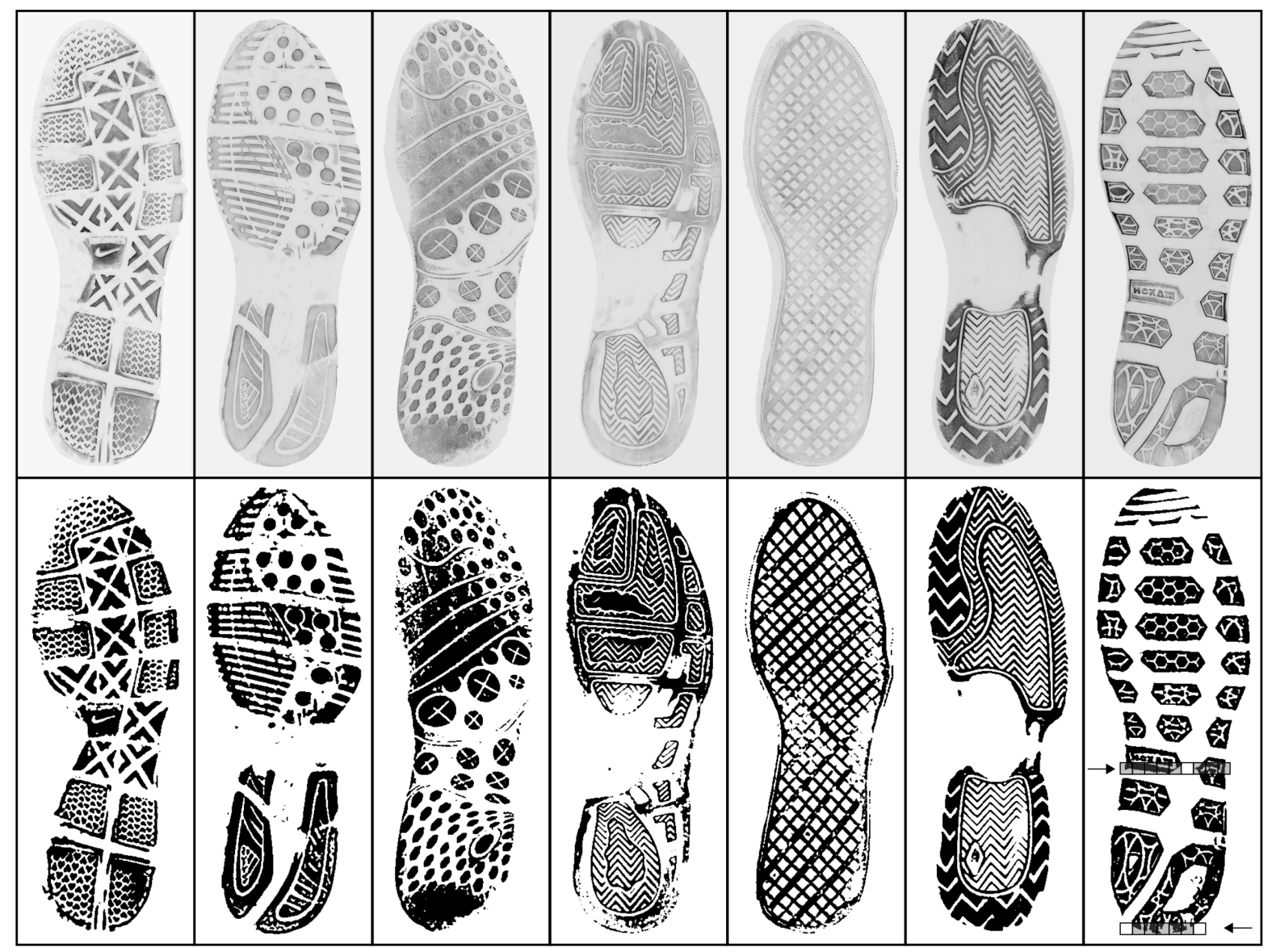

Figure 3: Handiprints (top) and tread element binary masks (bottom). From left to right: Nike Free TR, Men's size 9.5 (moderate wear); Adidas ${ }^{\circledR}$ adiPRENE, Men's size 10 (light wear); US Polo Assn. ${ }^{\circledR}$ (style unavailable), Men's size 9 (high wear); Nike Air Jordan XV1.5, Men's size 10.5, (moderate wear); Puma ${ }^{\circledR}$ (style unavailable), Men's size 9, (light wear), Nike Air Max Dragon, Men's Size 10 (light wear); Hoka One One ${ }^{\circledR}$ Conquest, Men's Size 11 (light wear). The arrows highlight two rows of cells in the heatmap, and the degree of shading within each cell indicates whether or not the example shoe increments $n$ (gray: increment cell, white: not a candidate).

mine the number of pixels on the outsole with tread in contact with the ground within each of the 987 spatial cells of the heatmap. For each cell, if the ratio of the number of pixels in the cell with tread on ground (for a given shoe) was greater than or equal to $25 \%$ of the cell's actual area $\left(25 \mathrm{~mm}^{2}\right.$ for full cells), then the shoe was considered a candidate. Moreover, if a 
RAC of a specific geometric shape was actually present on a shoe in this cell, then the cell's count was automatically incremented for the shoe in question, whether or not the criteria of $25 \%$ was met. For cell $(16,50), 927$ shoes had at least $6 \mathrm{~mm}^{2}$ of tread in contact with the ground at this location, or if less than $6 \mathrm{~mm}^{2}$ in contact, they actually exhibited a RAC at this location.

Using these estimates of contact, the row labeled "Chance of RAC Co-Occurrence" was populated. This row reports the empirical frequency of selecting two shoes at random and finding at least one positional match at a specific location, or the number of ways you can select two unrelated shoes from the dataset and find that they both possess RAC ' $A$ ' at location ' $B$.' To obtain this estimate, each spatial cell in the dataset was queried to determine (empirically) the number of times two unrelated shoes exhibited a pair of RACs with positional similarity, resulting in 987 cell-specific frequencies $(\times$ three shape categories + one category that ignores shape). Letting $n$ equal the number of "Shoes with Tread Contact in Cell," the number of ways two shoes $(k=2)$ can be selected (using a simple combination when order does not matter, but repetition is not allowed), was computed according to Eq. 4 (which equals 844,350 for $n=1,300$ ).

$$
\text { All Possible Pairs }=\frac{n !}{(n-k) ! k !}
$$

For cell $(16,50), 927$ of the 1,300 outsoles in this dataset either had $25 \%$ or more of their tread in contact with the ground, or a RAC (of any shape) detected at this location. If the number of times two unrelated shoes exhibited a pair of RACs (irrespective of shape categorization) with positional similarity for this cell is equal to 1,830 (empirically assessed), then there is a 1 in 234 chance of RAC co-occurrence in this $(927 ! /(927-2) ! 2 !)=429,201$ and $429,201 / 1,830=\lfloor 234.5\rfloor)$. Similarly, there were 925 shoes out of 1,300 with tread coverage equal to or greater than $25 \%$, or with a compact RAC present in this cell, and if the number of times two unrelated shoes exhibited a pair of compact RACs with positional 
similarity is equal to 276 (found empirically), there is a 1 in 1,548 chance of compact RAC co-occurrence in this cell $(925 ! /(925-2) ! 2 !=427,350$ and $427,350 / 276=\lfloor 1,548.4\rfloor)$.

Despite these positional and categorical random associations, it must be recognized that RACs with positional co-occurrence and identical shape categorizations are not necessarily geometrically similar. For example, two linear elements could vary in orientation, length, thickness, curvature, etc. Thus, the actual chance association must be modified to account for the true geometry of the features that co-occur in location and category, which is the primary thesis of this research effort. In other words: Do the two RACs under comparison have sufficient visual similarity to be considered indistinguishable and therefore reach a forensically significant chance association? In order to evaluate this, a visual comparison of co-occurring RACs was undertaken. However, based on the database size, if every pairwise comparison was visualized, the end result would be 3,227,855 comparisons (for all co-occurring RACs, across 1,300 outsoles, occupying 987 cells)! Given such an unrealistic task, a data-reduction technique was sought; instead of looking at every pair in a cell, the goal was to implement a three-pronged approach, beginning with (i.) a visual comparison of the most similar pairs (i.e., the 'close non-matches'), (ii.) computation of an estimate of the probability of visual indistinguishability given a specific mathematical score, and finally, (iii.) to obtain a numerical estimate of the probability of indistinguishability for the remaining non-matches in the cell. Given this approach, the first goal was to determine and sort close non-matches using a mathematical metric of similarity for RACs with coincidental positional similarity. In order to determine this mathematical similarity, the Fourier descriptor (FD) for each feature was computed. This was obtained by treating each RAC as a closed planar figure [21-23], and tracing the contour of the shape $(x(t), y(t))$ (where $t=0, \ldots N-1$ with $N=350$ ) before re-expression in a complex plane $z(t)=x(t)+i y(t)$ (where $i=\sqrt{-1}$ ). The resulting one-dimensional complex sequence of numbers was mapped to the frequency domain via the 
discrete Fourier transform [22] shown in Eq. 5, where $A_{m}$ and $\sigma_{m}$ are the magnitude and phase of the $m^{\text {th }}$ coefficient, respectively [22]:

$$
\begin{aligned}
Z(m) & =\sum_{t=0}^{N-1} z(t) e^{(-i 2 \pi m t / N)}=A_{m} e^{i \sigma_{m}} \\
m & =-N / 2, \ldots,-1,0,1, \ldots, N / 2-1
\end{aligned}
$$

The resulting coefficients were transformed to ensure invariance to translation and contour/sequence start point according to the following modifications [22]:

$$
\begin{array}{ll}
\hat{Z}(0)=0 & \Rightarrow \text { translation invariance } \\
\hat{\sigma}_{m}=\sigma_{m}-\frac{\sigma_{(-1)}+\sigma_{(1)}}{2} \hat{\sigma}_{m}=\sigma_{m}+m \frac{\sigma_{(-1)}-\sigma_{(1)}}{2} & \Rightarrow \text { start point invariance }
\end{array}
$$

An example of two RACs from cell $(16,50)$ and their associated Fourier descriptors can be seen in Fig. 4 (note that the axes for the FD images are arbitrarily labeled since the FD process creates normalized figures).
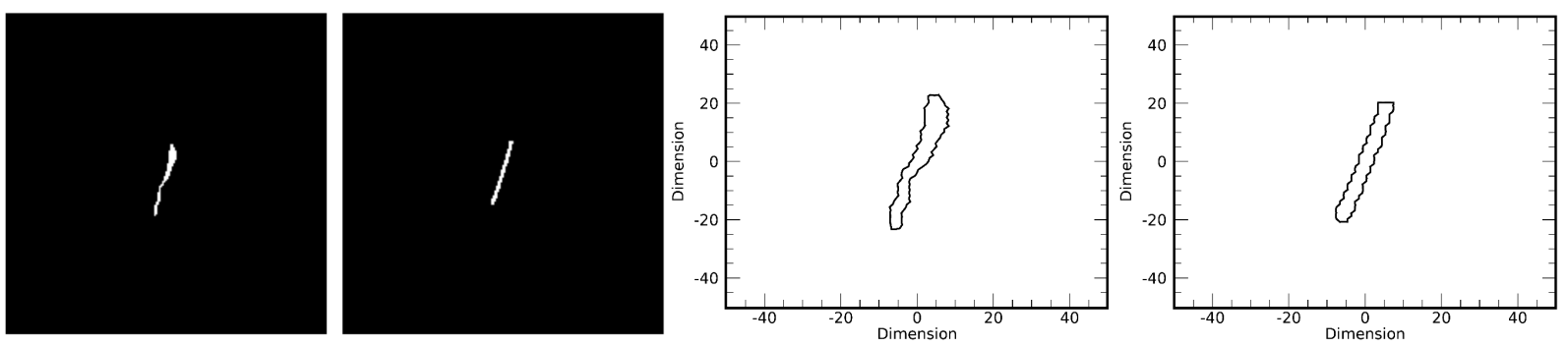

Figure 4: Two linear/elongated RACs (left) from different shoes that co-occur in cell $(16,50)$, and their associated Fourier descriptors (right). Note that the axes for the FD images are arbitrary dimensions.

Using the resulting FD features, RAC similarity was computed using four different metrics: matched filter (MF), modified cosine similarity (MCS), Hausdorff distance (HD), and average Euclidean distance (ED) [24]. Using the actual RAC images, modified phase only correlation (MPOC) was likewise computed (please see [24] for a previously published description of each metric). Using crime scene-like RACs (also previously prepared and described 
[24]), each accidental was compared to both high quality known matches (KMs) and high quality known non-matches (KMNs) with positional association within a $5 \mathrm{~mm} \times 5 \mathrm{~mm}$ cell around the crime scene-like RAC's centroid. This resulted in 44,230 chance associations in position, and based on feature density, each crime scene-like RAC was compared to an average of $72 \pm 21$ (one standard deviation) other RACs, with a maximum of 126 comparisons and a minimum of 15 comparisons [25]. In total, 8,830 KM and 755,380 KNM pairwise comparisons were computed $(\mathrm{KMs}=1,766 \times 5$ metrics $=8,830$ and $\mathrm{KNMs}=151,076 \times 5$ metrics $=755,380)$. The resulting similarity scores were evaluated using cumulative match characteristic (CMC) curves, receiver operating characteristic (ROC) curves, and area under the curve (AUC) integrals [25]. Based on all available results (please see [25] for figures), HD was selected as the metric to use moving forward (i.e., it exhibited the fastest rising CMC, and based on AUCs, it was statistically significantly better than all other metrics (at $p<0.05$ ) [26] except average Euclidean distance $(\mathrm{HD} \mathrm{AUC}=0.8152$ and $\mathrm{ED}$ AUC $=$ $0.8151))[25]$

Since HD was found to be superior (or equal to ED), its computation is briefly summarized here; this metric was obtained by first computing the Euclidean distance $(d(a, b))$ between a point $\left(e . g ., a_{1}\right)$ on the perimeter of $\mathrm{RAC}(A)$ and all points on the perimeter of $\mathrm{RAC}(B)$ (as illustrated in Fig. 5 (left)), retaining the smallest distance from $a_{1}$ to $B$. Next, this process was repeated for all points on $A$ (i.e., $\left.a_{2} \ldots a_{n}\right)$, wherein $h(A, B)$, or the maximum of these minimum distances, was retained [27]. This same process was repeated to compare all points on RAC perimeter $B$ to those on RAC perimeter $A$, thus obtaining $h(B, A)$. Finally, the actual Hausdorff distance was defined as the maximum of these two values $(h(A, B)$ and $h(B, A))$ as illustrated in Eq. 7.

$$
H D(A, B)=\max \{h(A, B), h(B, A)\}
$$

$$
\text { where } h(A, B)=\max _{a \in A}\left\{\min _{b \in B}\{d(a, b)\}\right\}
$$


Next, the HD similarity/dissimilarity measure was used to rank RAC pairs. This was accomplished by first computing the HD for 6,993 KM comparisons (of which 5,227 were based on high quality versus high quality RAC comparisons (either marked by different analysts, or the same analyst repeatedly while assessing inter- and intra-analyst variability)), and the remaining 1,766 were based on high quality versus crime scene-like RAC comparisons as previously mentioned. Finally, the HD dissimilarity of 3,227,855 KNM comparisons was computed based on all RACs in the database with positional association available at the time of analysis. Using the maximum and minimum HD dissimilarity value computed from all datasets, the scores were normalized such that a HD score of 1.0 signified indistinguishable RACs (within this database and the resolution possible using Hausdorff distance), and a score of 0.0 was associated with the most dissimilar pairwise comparison between two known non-match RACs in this dataset.

Using this metric as a mathematical measure of similarity to sort the pairs from most similar to least similar, the 15 most-similar RAC KNM pairs with positional similarity (per spatial cell) were visually examined to ascertain the likelihood of genuine chance associations $(15 \times 987$ cells $\times 4$ shape classes [modified by cells with less than 15 pairs] $=55,445$ visual comparisons of which 46,336 were unique).

Following analysis of the paired RACs based on HD scores, a second investigation was undertaken to determine if total Euclidean (TE) distance (rather than average Euclidean distance as previously measured and found to be comparable to HD), and/or the percent area $(\% \mathrm{~A})$ of overlap between RACs might impact the chance of a false association. As used here, the total Euclidean distance (TE) was defined as a measure of the displacement needed to transform one shape into another, as illustrated in Fig. 5 (middle), which depicts three distance measurements at corresponding feature vector locations (triangles and squares) on two different RACs. This distance computation was computed for all $350(\mathrm{x}, \mathrm{y})$ coordinates 
on each RAC FD, starting at an angle of $0^{\circ}$ and moving counterclockwise. The final measure to transform between each shape was defined as the total Euclidean distance from mated points, as illustrated in Eq. 8.

$$
\mathrm{TE}(A, B)=\sum_{i=1}^{350} \sqrt{\left(x_{a_{i}}-x_{b_{i}}\right)^{2}+\left(y_{a_{i}}-y_{b_{i}}\right)^{2}}
$$

The last measure of similarity was a simple computation of the percent area correspondence between the RACs being compared (Eq. 9), computed on binary RAC images, but illustrated using FDs in Fig. 5 (right), where the darkest shade of gray emphasizes the physical overlap.

$$
\% \mathrm{~A}(A, B)=\frac{[\text { area of overlap }] \times 2}{[\text { area of } \mathrm{A}+\text { area of } \mathrm{B}]} \times 100 \%
$$

Hausdorff Distance

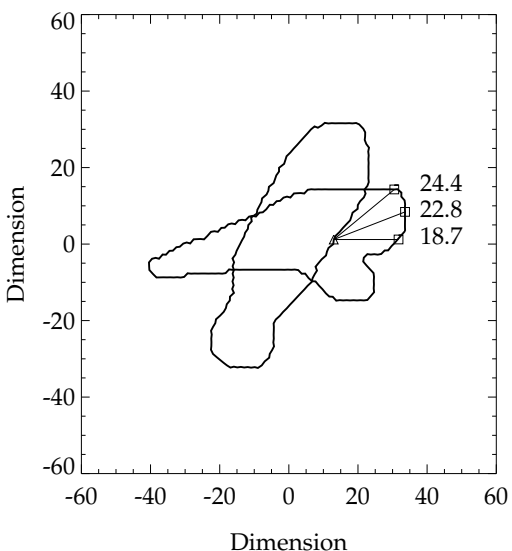

Total Euclidean Distance

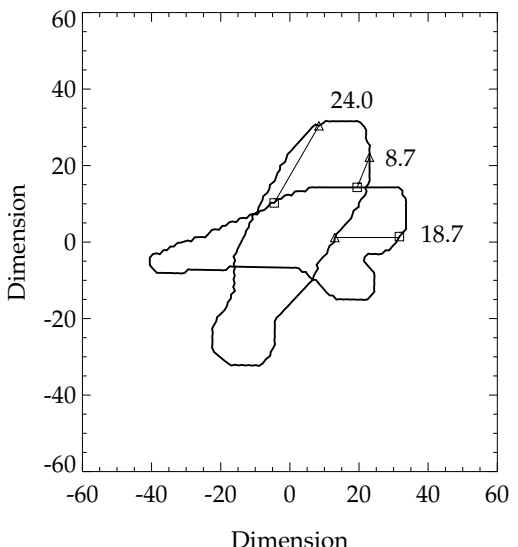

Area Overlap

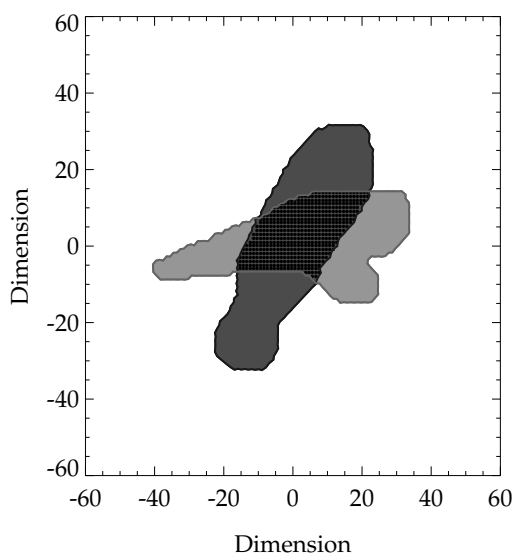

Figure 5: Illustration of computation of Hausdorff distance (left; for $a_{1}$ versus $b_{1}, b_{10}$ and $b_{25}$ ), total Euclidean distance (middle; for three corresponding features at $a_{1}$ vs. $b_{1}, a_{50}$ vs. $b_{50}$ and $a_{100}$ vs. $b_{100}$ ), and percent area overlap (right; darkest shaded region).

The remaining three similarity metrics of interest, HD (assessed as slightly superior to MF, MCS, ED and MPOC based upon past work [25]), along with the newer addition of 
TE and \%A, were used to compare 4,865 high quality versus high quality replicate known matches (KM), and all associated known non-matches (KNM) with positional co-occurrence, totaling 440,523 KNM comparisons. This data was used to create additional cumulative match characteristic curves as illustrated in Fig. 6 (left). Receiver operating characteristic curves were also computed for the $4,865 \mathrm{KMs}$, but this time expanding the KNMs to include the entire dataset, totaling $3,668,378$ KNMs $(3,227,855$ heatmap-based KNMs available at the time of analysis, as a function of 1,300 shoes, divided between 987 cells, but restricted to RACs originating from different shoes, plus 440,523 additional high quality KNMs from replicate RAC markings), as illustrated in Fig. 6 (right). In addition, the integral for each ROC curve was computed and found to be statistically significantly different $(p<0.05)$ [26] at 0.842 for HD, 0.811 for TE and 0.864 for \%A; the results indicate that \%A slightly outperformed HD and TE with regard to high quality imagery as a function of the higher AUC (although both HD and \%A had comparable CMCs).

However, when these three metrics are used to compare crime scene-like imagery versus high quality marked RACs (1,625 KMs and 140,861 KNMs for the CMC curves and 1,625 KMs and 3,368,716 KNMs for the ROC curves - again, derived from 3,227,855 heatmapbased KNMs available at the time of analysis, as a function of 1,300 shoes, divided between 987 cells, but restricted to RACs originating from different shoes, plus 140,861 crime scenelike KNMs), the results indicated a mixed response. In other words, HD was superior based on $\mathrm{CMC}$ and $\mathrm{ROC}$ data; the CMC curve for HD rises more quickly than for the other two compared metrics, and the AUCs were statistically significantly different $(p<0.05)[26]$ at 0.815 for $\mathrm{HD}, 0.707$ for TE and 0.790 for $\% \mathrm{~A}$ (note that $p=2.902 \mathrm{e}-05$ for HD versus $\% \mathrm{~A}$ ).

The cumulative interpretation is that HD is superior when comparing crime scene-like imagery to high quality imagery, but that $\% \mathrm{~A}$ is somewhat superior to HD when comparing high quality RACs to other high quality RACs (significantly greater AUC, but comparable 


\section{Cumulative Match Characteristic Curve}

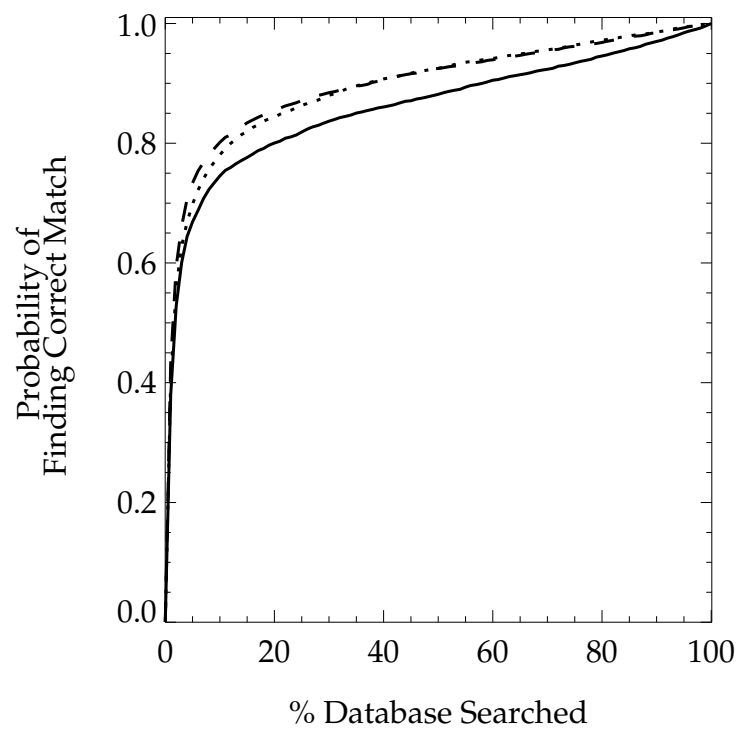

Receiver Operating Characteristic Curve

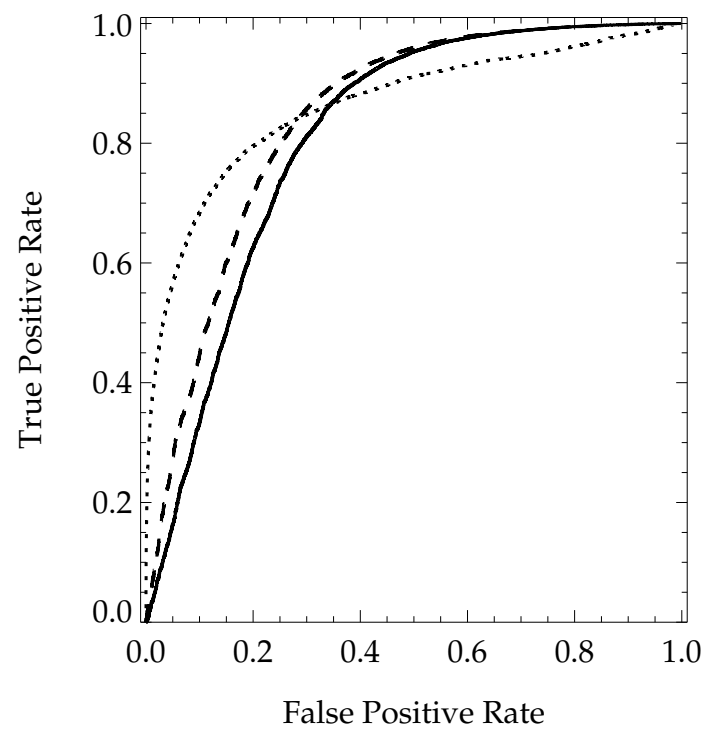

Figure 6: CMC curves (left) for high quality duplicate marked RACs for HD (dashed line), TE (solid line) and \% A (dotted line). Note that the $\mathrm{x}$-axis represents the percent database searched in order to encounter a RAC's KM. The plot at right depicts the ROC curves for the same similarity metrics; the resulting AUCs are all statistically significantly different $(p<0.05)[26]$ at 0.842 for $\mathrm{HD}, 0.811$ for TE and 0.864 for \% A.

CMCs). Given this mixed response, the process of visually comparing RACs was repeated using \% A as the mathematical measure of similarity to sort the pairs from most similar to least similar, and this time, expanding the list to include the top 25 most-similar RAC KNM pairs with positional similarity (per spatial cell) $(25 \times 987$ cells $\times(3$ shape classes +1 class ignoring shape) [modified by cells with less than 25 pairs] $=91,607$ visual comparisons performed in duplicate by two analysts). Note that part of the motivation to consider similar pairs using \% A (in addition to HD) is because the known non-match database is all high quality imagery, and it is likely that several different pairs will be visually evaluated if a new metric is used for sorting, thus expanding the total number of features visually reviewed.

In addition, it should be noted that the research group also updated the rules to define 


\section{Cumulative Match Characteristic Curve}

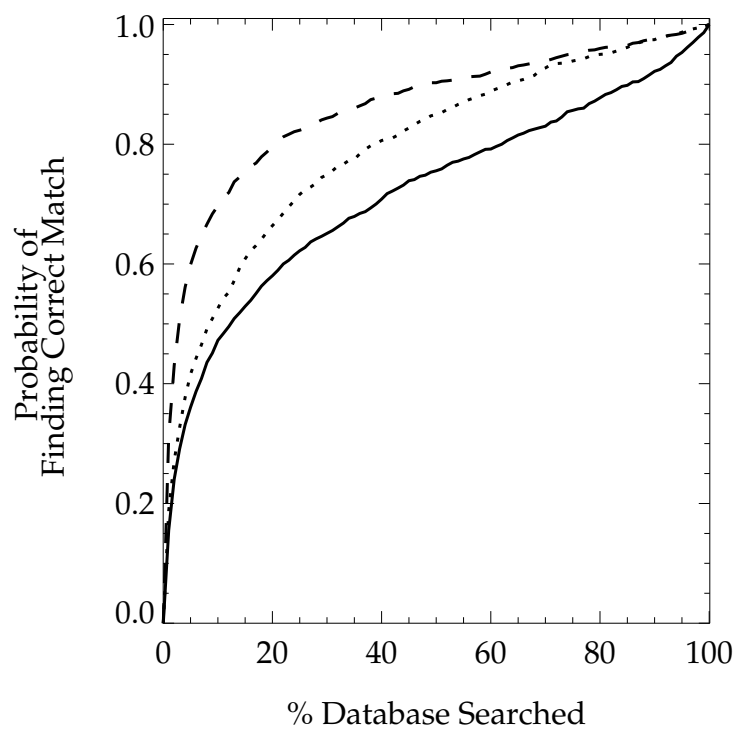

Receiver Operating Characteristic Curve

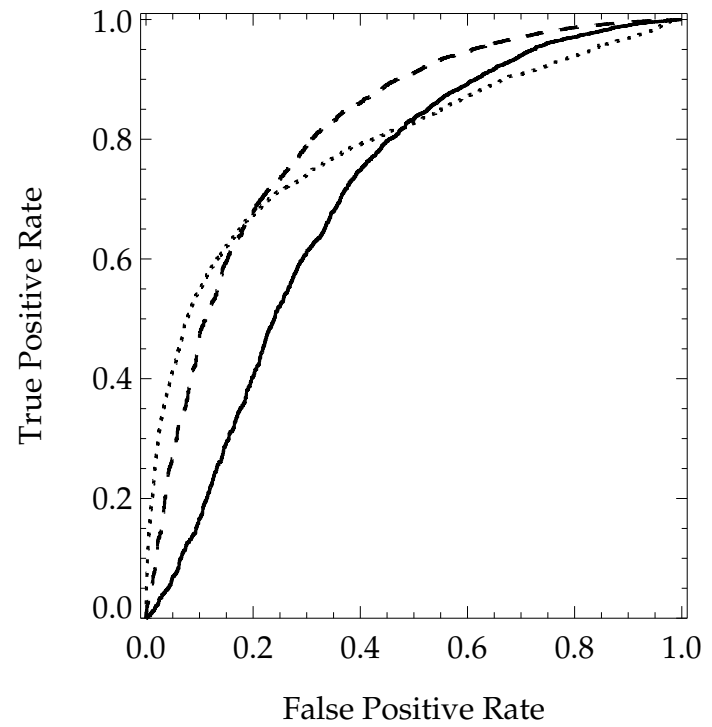

Figure 7: CMC curves (left) for crime scene-like versus high quality marked RACs for HD (dashed line), TE (solid line) and \% A (dotted line). Note that the $\mathrm{x}$-axis represents the percent database searched in order to encounter a RAC's KM. The plot at right depicts the ROC curves for the same similarity metrics; the resulting AUCs are all statistically significantly different $(p<0.05)[26] 0.815$ for HD, 0.707 for TE and 0.790 for $\% \mathrm{~A}$ (note that $p=2.902 \mathrm{e}-05$ for $\mathrm{HD}$ versus $\% \mathrm{~A}$ ).

shape-classification. The motivation for this was two-fold. First, a few classification anomalies were noted (i.e., an elongated feature that ended up being defined as variable since its aspect-ratio was not large enough to meet the elongated/linear categorization). Second, and more problematic, was the fact that small features were variably classified as irregular or isometric, depending on their complexity. This most likely resulted from earlier classification definitions that allowed the irregular category to serve as a catch-all for anything that was not elongated, triangular, rectangular or circularly-shaped (wherein the latter three were grouped together and formerly termed isometric). However, given that "real" RACs rarely conform to any stylized shape (which means shape classification varies between human- 
observers, and between algorithmic-rules and human-perception — as previously discussed in [20]), and the observation that small features could end up being classified as irregular if they were not sufficiently circular, sufficiently triangular, nor sufficiently rectangular to make it past the isometric classification system as summarized in [20], an update to the categorization system was deemed relevant. Thus, a decision was made to recast the shapes into three categories, defined as variable, linear and compact. The end result required 12 classification rules, such that minute RACs (10 pixels $<0.5 \mathrm{~mm})$, excluding thin lines, and those with low complexity are deemed compact, curvy elongated RACs with protrusions as variable (rather than linear), and restricting the linear label to RACs that are long and very thin (additional details regarding the classification rules can be found in the supplementary materials section that accompanies this paper).

Using both \% A and the new shape categorizations, 91,607 additional visual comparisons were completed to determine chance association. After assessing the 55,445 visual comparisons based on HD and the previous shape classification system (of which 46,336 were unique), and the additional 91,607 visual comparisons based on $\% \mathrm{~A}$ and the new shape classifications system, a total of 114,238 unique visual comparisons were evaluated $(22,631$ unique based on HD, 67,902 unique based on \% A and 23,705 RAC pair repeats across both HD and $\% \mathrm{~A})$.

\section{Results and Discussion}

When comparing RACs deemed mathematically similar by HD (55,445 visual comparisons performed in duplicate by two different analysts), the criteria for indistinguishability was extremely stringent, such that RAC pairs were defined as "indistinguishable" if they exhibited minimal pixel-level differences (Fig. 8, top - note that visual comparisons were conducted using both raw RAC and FD images, but only FD images are illustrated here). 
This resulted in a total of 227 indistinguishable pairs for the first analyst, a total of 178 indistinguishable pairs for the second analyst, and an overlap of 58 pairs (e.g., 58 of the 227 pairs deemed indistinguishable by the first analyst were also deemed indistinguishable by the second analyst). For these classifications, inter-rater reliability was evaluated using Gwet's $A C_{1}$ and Aickin's $\hat{\alpha}_{A}$ (with convergence after 110 iterations), producing coefficients of 0.995 and 0.912 , respectively [28]. However, the indistinguishability results presented using HD do not necessarily encompass the range of reasonable variation in RAC reproduction that can be expected when reproducing and marking RACs in replicate test impressions [29]. Thus, the second analysis ( of 91,607 visual comparisons of RACs mated using \%A, also preformed in duplicate by two analysts) expanded the definition of indistinguishabilty to include RACs with increasing variation (e.g., minimal pixel-level variation, plus minor variations in length, width, orientation, etc. that can be expected when comparing knownmatch replicate test impressions, and therefore reasonably explainable variations that can be expected when comparing known-match test and questioned impressions) (Fig. 8, bottom).

Using this more permissive criteria for an association, a total of 1,589 indistinguishable pairs were detected by the first analyst, a total of 1,524 indistinguishable pairs by the second analyst, and an overlap of 931 pairs (e.g., 931 of the 1,589 pairs deemed indistinguishable by the first analyst were also deemed indistinguishable by the second analyst). For these classifications, inter-rater reliability was evaluated using Gwet's $A C_{1}$ and Aickin's $\hat{\alpha}_{A}$ (with convergence after 65 iterations), producing coefficients of 0.986 and 0.917 , respectively [28].

The total combined 2,182 indistinguishable features based on sorted \% A similarity were comprised of 1,213 linear, 850 compact and 39 variable RAC pairs (the remaining 80 were mixed classes, for example, a linear feature compared to a variable feature). For each shape category, the empirical observations of indistinguishability were treated as binary outcomes $y_{i, j}$ (equal to 1 when RAC pair $i$ and $j$ were indistinguishable, and 0 when RAC pair $i$ 

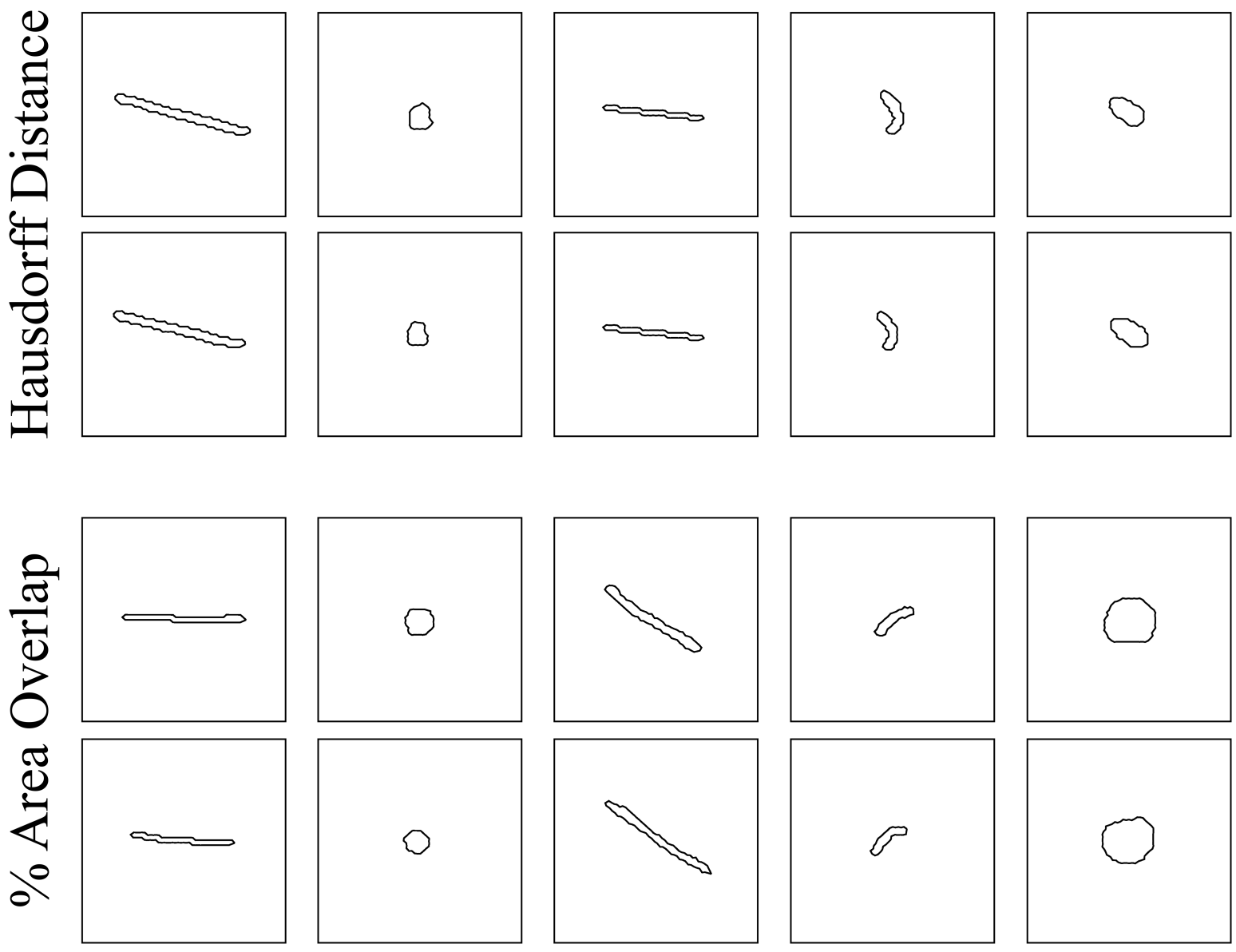

Figure 8: Examples of KNM RACs mated as similar using HD (top) and \%A (bottom) and deemed visually confusing by analysts. Note that indistinguishability for $\% \mathrm{~A}$ are more permissive, allowing for greater variations in size, angle, thickness, boundary, etc. that might result when comparing replicate test impressions and/or test versus questioned impressions.

and $j$ were distinguishable), and modeled as a Bernoulli distribution with the probability of indistinguishability equal to $p_{i, j}$. Using hold-one-out-cross-validation, a binary logistic regression was defined to model $p_{i, j}$ including polynomials up to the $K^{\text {th }}$ degree as illustrated in Eq. 10 (with $\mathrm{K}=4$ for linear features, and 3 for compact and variable features) [30]:

$$
\begin{gathered}
y_{i, j} \sim \operatorname{Bernoulli}\left(p_{i, j}\right) \\
31
\end{gathered}
$$




$$
\operatorname{logit}\left(p_{i, j}\right)=\alpha_{0}+\sum_{k=1}^{K} \alpha_{k} \times\left(\% A_{i, j}\right)^{k}
$$

Models with increasing polynomials were compared using three methods; the Akaike information criterion (AIC), the Hosmer-Lemeshow (H-L) statistic, and receiver operating characteristic (ROC) curves. Selecting the model with the lowest AIC value maximizes the probability that the candidate model describes the empirical data, but with a penalty for lack of parsimony [31]. The AIC for each polynomial can be transformed into an estimate of the relative likelihood of the model, and normalized by the sum of the likelihoods for all other proposed models to obtain weights $w_{k}(\mathrm{AIC})[31]$. The largest $w_{k}(\mathrm{AIC})$ weight is the best model candidate of all proposed (or $\left.w_{k=b e s t}(\mathrm{AIC})=\max \left(w_{k}(\mathrm{AIC})\right)\right)$ and the ratio of $w_{k=\text { best }}(\mathrm{AIC}) / w_{k}(\mathrm{AIC})$ for each model with more or fewer terms is the number of times the best-fitting model is more likely than another model $w_{k}(\mathrm{AIC})$, denoted as $\mathrm{L}(\mathrm{AIC})$ in Table 2.

Additionally, the inclusion of increasing polynomials was evaluated using the H-L goodness of fit test, wherein observed and predicted probabilities were compared using a $\chi^{2}$ statistic such that $p$-values greater than 0.05 indicate a "good fit" (i.e., one fails to reject the null hypothesis that the observed and predicted probabilities are the same) [32].

Finally, using the number of terms associated with the best-fit model and hold-one-outcross-validation in order to estimate the coefficients for Eq. 10, the probability of indistinguishability for each held-out observation was sequentially predicted in order to model the probability density associated with indistinguishable and distinguishable RAC pairs as a function of match score $(\% \mathrm{~A})$. The resulting predicted probability densities and empirically reported densities were used to create receiver operating characteristic (ROC) curves and the area under the curve (AUC) for each geometric category (linear, compact and variable) was computed. As illustrated in Table 3, all p-values [26] were greater than 0.05, further 
Table 2: Table of AIC and $p$-values describing the fit for models with increasing polynomials. The shaded cells reflect the best fit model for linear, compact and variable shaped RACs, and L(AIC) describes the number of times the best-fit model is more likely to be a better fit than a lower- or higher-order polynomial. Equivalently, the $p$-value describes the probability that the observed and modeled/predicted values are a good fit according to the H-L $\chi^{2}$ statistic; $p$-values greater than 0.05 indicate a good fit. Based on both the AIC and H-L goodness of fit, a fourth-order polynomial was selected for linear features, and a third-order for compact and variable RACs.

\begin{tabular}{|c|c|c|c|c|c|c|c|c|c|c|c|c|c|c|c|}
\hline Polynomial & \multicolumn{3}{|c|}{ First-Order } & \multicolumn{3}{c|}{ Second-Order } & \multicolumn{3}{c|}{ Third-Order } & \multicolumn{3}{c|}{ Fourth-Order } & \multicolumn{3}{c|}{ Fifth-Order } \\
\hline Metric & AIC & L(AIC) & $\boldsymbol{p}$-value & AIC & L(AIC) & $\boldsymbol{p}$-value & AIC & L(AIC) & $\boldsymbol{p}$-value & AIC & L(AIC) & $\boldsymbol{p}$-value & AIC & L(AIC) & $\boldsymbol{p}$-value \\
\hline Linear & 6,887 & $3.63 \mathrm{e} 04$ & $3.57 \mathrm{e}-06$ & 6,876 & 148 & 0.0011 & 6,872 & 20.1 & 0.0145 & 6,866 & 1.00 & 0.1374 & 6,866 & 1.00 & 0.1118 \\
\hline Compact & 5,965 & $2.17 \mathrm{e} 09$ & $7.23 \mathrm{e}-07$ & 5,935 & 665 & 0.0177 & 5,922 & 1.00 & 0.5719 & 5,923 & 1.65 & 0.7063 & NA & NA & NA \\
\hline Variable & 500 & $5.91 \mathrm{e} 09$ & $4.48 \mathrm{e}-09$ & 482 & $7.29 \mathrm{e} 05$ & $2.80 \mathrm{e}-05$ & 455 & 1.00 & 0.2184 & 457 & 2.72 & 0.6884 & NA & NA & NA \\
\hline
\end{tabular}

confirming a failure to detect statistical differences between the predictions and the empirical observations.

Table 3: Area under the curve for receiver operating characteristic curves for empirical and predicted indistinguishability outcomes as a function of $\% \mathrm{~A}$. Note that the empirical report of indistinguishability was used to define ground truth, and all $p$-values [26] are greater than 0.05 , indicating agreement (or a failure to detect disagreement) between the modeled and empirical observations.

\begin{tabular}{|c|c|c|c|}
\hline Feature & Empirical AUC & Predicted AUC & $p$-value \\
\hline Linear & 0.8640 & 0.8648 & 0.9095 \\
\hline Compact & 0.8712 & 0.8708 & 0.9671 \\
\hline Variable & 0.8141 & 0.8821 & 0.1677 \\
\hline
\end{tabular}

Using all available data, Table 4 reports the best-fit coefficients for Eq. 10 and Fig. 9 plots the decision outcome $y_{i, j}$ regarding visual similarity for each RAC comparison. For linear features, there were 1,213 pairs considered visually confusing $\left(y_{i, j}=1.0\right.$, but jittered \pm 0.05 in the $y$-direction for visual clarity), and 21,037 deemed visually differentiable $\left(y_{i, j}=0\right.$, also jittered \pm 0.05 for visual clarity). Similarly, 850 compact pairs (and 39 variable) were deemed 


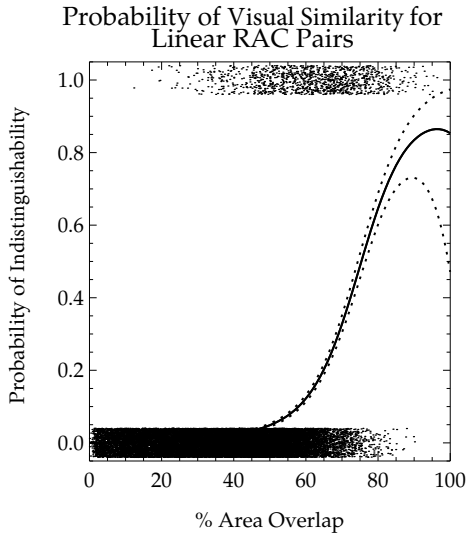

visually confusing, while 35,265 compact pairs (and 23,864 variable pairs) were classified as differentiable. Using these estimates, the conditional probability of indistinguishability (I) given a $\% \mathrm{~A}$ score $(p$ (indistinguishability $\mid \% \mathrm{~A})$ or $p(\mathrm{I} \mid \% \mathrm{~A}))$ was modeled (solid line), along with the $95 \%$ credible interval (dashed lines) [30].

Table 4: Estimated coefficients for Eq. 10, used to predict the probability of indistinguishability based on \%A for linear, compact and variable RAC pairs.

\begin{tabular}{|c|c|c|c|c|c|}
\hline Coefficient & $\alpha_{0}$ & $\alpha_{1}$ & $\alpha_{2}$ & $\alpha_{3}$ & $\alpha_{4}$ \\
\hline Linear & -11.89 & 0.5240 & -0.0141 & $1.844 \mathrm{e}-4$ & $-8.228 \mathrm{e}-7$ \\
\hline Compact & -120.6 & 4.374 & -0.0577 & $2.657 \mathrm{e}-4$ & NA \\
\hline Variable & -65.42 & 2.909 & -0.0479 & $2.598 \mathrm{e}-4$ & NA \\
\hline
\end{tabular}

Figure 9: Plot of probability of indistinguishability (solid line) and 95\% credible intervals (dotted lines) versus match score $(\% \mathrm{~A})$ for linear, compact and variable RAC pairs. The solid dots (jittered vertically \pm 0.05 for ease in visualization) are the empirically assigned $y_{i, j}$ responses for indistinguishable (1.0) and distinguishable (0.0) pairs.
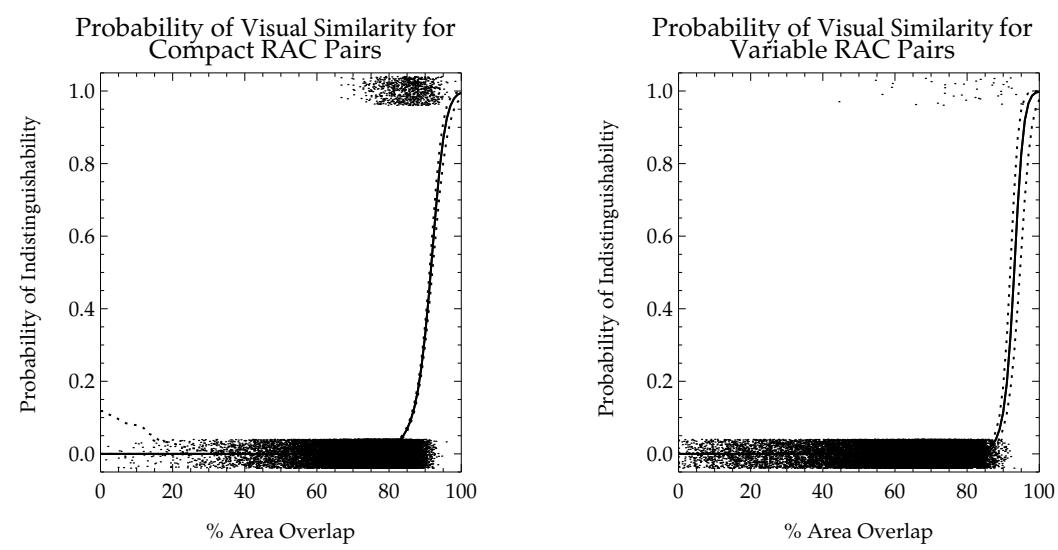

Using the resulting $p(\mathrm{I} \mid \% \mathrm{~A})$ model, the chance indistinguishability of the remaining pairwise RAC comparisons in each spatial cell were estimated. For example, spatial cell $(16,50)$ 
contains 17 linear features, resulting in $n(n-1) / 2=136$ possible linear-RAC pairs. If the visual assessment of the 25 most similar non-matching RACs resulted in 1 RAC pair classified as visually indistinguishable based on empirical observation, then there are an additional 111 pairs that are mathematically less similar based on sorting, but not visually/empirically assessed. However, the probability of visual indistinguishability given the computed mathematical similarity $p(\mathrm{I} \mid \% \mathrm{~A})$ as modeled using the predictions from Fig. 9 allows for an estimate of the remaining count of possible indistinguishable pairs in the cell according to Eq. 11. In this example, if the summation shown in Eq. 11 predicts another 0.1238 RAC "pairs" with visual indistinguishability, then there is a 1.1238 in 136 (or 1 in 121) chance of visual indistinguishability for all pairwise comparisons at this spatial location.

$$
\begin{aligned}
p(\mathrm{I} \mid \text { co-occurrence }) & =\frac{\text { Indistinguishable }+\sum_{i, j=26}^{N=136} p\left(\mathrm{I} \mid \% \mathrm{~A}_{i, j}\right)}{N=136} \\
& =\frac{1+0.1238}{136}
\end{aligned}
$$

Given an empirical frequency of chance co-occurrence in position of 136 out of 425,503 $(923 ! /(923-2) ! 2 !=425,503)$, then $p($ co-occurrence $)=3.20 \mathrm{e}-04$. Finally, the probability of both co-occurrence in position and indistinguishability, equal to the intersection $p($ co-occurrence $\cap \mathrm{I})$, can be computed according to Eq. 12, and for this example equals a chance of 1 in 378,621 pairs (assuming that $p(\mathrm{I} \mid \% \mathrm{~A}$ ) does not vary with spatial location).

$$
\begin{aligned}
p(\text { co-occurrence } \cap \mathrm{I}) & =p(\text { co-occurrence }) \times p(\mathrm{I} \mid \text { co-occurrence }) \\
& =\frac{136}{425,503} \times \frac{1.1238}{136} \\
& =\frac{1.123823}{425,503}=2.64 \mathrm{e}-06 \\
& =1 \text { in } 378,621
\end{aligned}
$$


Using the 91,607 empirical conclusions drawn for the "most-similar" RAC pairs, and estimating the probability of indistinguishability for all other co-occurring RACs in the database with the same geometric/shape categorization (linear, compact or variable) $(1,105,943$ $91,607=1,014,336)$, the intersection of co-occurrence and indistinguishability $p$ (co-occurrencen I) was computed for all spatial cells, and this information was added to the online interactive heatmap (www.4n6chemometrics.com/database/). This is illustrated in Fig. 10, which is the summary data for cell $(16,50)$ from Fig. 2, updated to include the chance indistinguishability, including a statement that reports the number of empirical and modeled (total less empirically evaluated) probabilities for indistinguishability used to generate the estimate.

\begin{tabular}{|c|c|c|c|c|}
\hline & Variable & Linear & Compact & Any Shape \\
\hline All RACs in Database & 28,236 & 21,363 & 22,707 & 72,306 \\
\hline All RACs in Cell & 25 & 17 & 27 & 69 \\
\hline Chance of Finding RAC in Cell & 1 in 2,892 & 1 in 4,253 & 1 in 2,678 & 1 in 1,047 \\
\hline Unique Shoes Contributing to RAC Count & 23 & 17 & 24 & 61 \\
\hline Shoes with Tread Contact in Cell & 925 & 923 & 925 & 927 \\
\hline Chance of RAC Co-Occurrence & 1 in 1,689 & 1 in 3,128 & 1 in 1,548 & 1 in 234 \\
\hline Chance of RAC Indistinguishability & 1 in $1,146,971$ & 1 in 378,621 & 1 in 151,038 & NA \\
\hline Visually Evaluated / Total RAC Pairs & $25 / 298$ & $25 / 136$ & $25 / 348$ & NA \\
\hline Similarities \& Likelihood Ratios & Variable & Linear & Compact & Any Shape \\
\hline
\end{tabular}

Figure 10: Table from Fig. 2 updated to include chance association estimate, and a statement to describe the number of empirical and modeled probabilities of indistinguishability to generate the estimate (e.g., 25 visual estimates out of a total of 136 possible linear pairs, leaving 111 predictions).

Summary statistics for chance co-occurrence and indistinguishability are provided in Table 5, (as per Eqs. 11 and 12), which describes the predicted or expected chance (solid line in Fig. 9), as well as the most conservative (worst-case scenario) estimate based on the upper 95\% credible interval for prediction (upper dotted line in Fig. 9).

The median estimate of chance indistinguishability for all cells based on expected val- 
Table 5: Summary statistics for chance of co-occurrence and indistinguishability, based on 91,607 visual-empirical estimates of the most similar mated RACs with spatial co-occurrence, and predicted for the remaining 1,014,336 cooccurring pairs (as per Eqs. 11 and 12). The column describing the predicted chance corresponds to the expected value (solid line in Fig. 9), which is accompanied by a conservative (worst-case scenario) estimate based on the upper 95\% credible interval for prediction (upper dotted line in Fig. 9).

\begin{tabular}{|c|c|c|c|c|c|c|}
\hline Geometry & \multicolumn{2}{|c|}{ Linear } & \multicolumn{2}{c|}{ Compact } & \multicolumn{2}{c|}{ Variable } \\
\hline Indistinguishability & $\begin{array}{c}\text { Expected } \\
(\mathbf{1} \text { in X) }\end{array}$ & $\begin{array}{c}\text { Conservative } \\
(\mathbf{1} \text { in X) }\end{array}$ & $\begin{array}{c}\text { Expected } \\
(\mathbf{1} \text { in X) }\end{array}$ & $\begin{array}{c}\text { Conservative } \\
(\mathbf{1} \text { in } \mathbf{X})\end{array}$ & $\begin{array}{c}\text { Expected } \\
(\mathbf{1} \text { in X) }\end{array}$ & $\begin{array}{c}\text { Conservative } \\
(\mathbf{1} \text { in X) }\end{array}$ \\
\hline Mean & $2.73 \mathrm{e} 08$ & $1.43 \mathrm{e} 07$ & $7.45 \mathrm{e} 39$ & $8.65 \mathrm{e} 05$ & $8.61 \mathrm{e} 29$ & $4.43 \mathrm{e} 08$ \\
\hline Median & $5.41 \mathrm{e} 05$ & $4.44 \mathrm{e} 05$ & $2.98 \mathrm{e} 06$ & $2.91 \mathrm{e} 05$ & $1.80 \mathrm{e} 07$ & $8.81 \mathrm{e} 05$ \\
\hline Min & $6.74 \mathrm{e} 04$ & $5.66 \mathrm{e} 04$ & $5.90 \mathrm{e} 04$ & $3.97 \mathrm{e} 04$ & $3.33 \mathrm{e} 05$ & $1.47 \mathrm{e} 05$ \\
\hline Max & $4.94 \mathrm{e} 10$ & $1.82 \mathrm{e} 09$ & $4.32 \mathrm{e} 42$ & $5.60 \mathrm{e} 07$ & $7.74 \mathrm{e} 32$ & $1.05 \mathrm{e} 11$ \\
\hline
\end{tabular}

ues is 1 in 541,276 for linear, 1 in 2,976,584 for compact and 1 in 18,031,824 for variable features. Similarly, the median estimate of chance indistinguishability for all cells based on the upper $95 \%$ credible interval for estimation is 1 in 444,126 for linear, 1 in 291,111 for compact, and 1 in 880,774 for variable features. The greatest chance based on the upper 95\% credible interval for estimation is 1 in 39,664 for two compact RACs. Figs. 11-13 are frequency histograms describing the chance association (intersection of spatial co-occurrence and indistinguishability), including the number of cells with zero associations, and the number with chance associations more rare than 1 in a million for the worst-case scenario (upper 95\% credible interval for estimation). Naturally, compact features have the fewest number of cells with chance association more rare than 1 in a $1,000,000$, owning to the fact that many of these features are best described as small and isometric, while variable pairs have the greatest number of cells with chance associations more rare than 1 in $1,000,000$, since these features tend to be large, and geometrically-complex. 


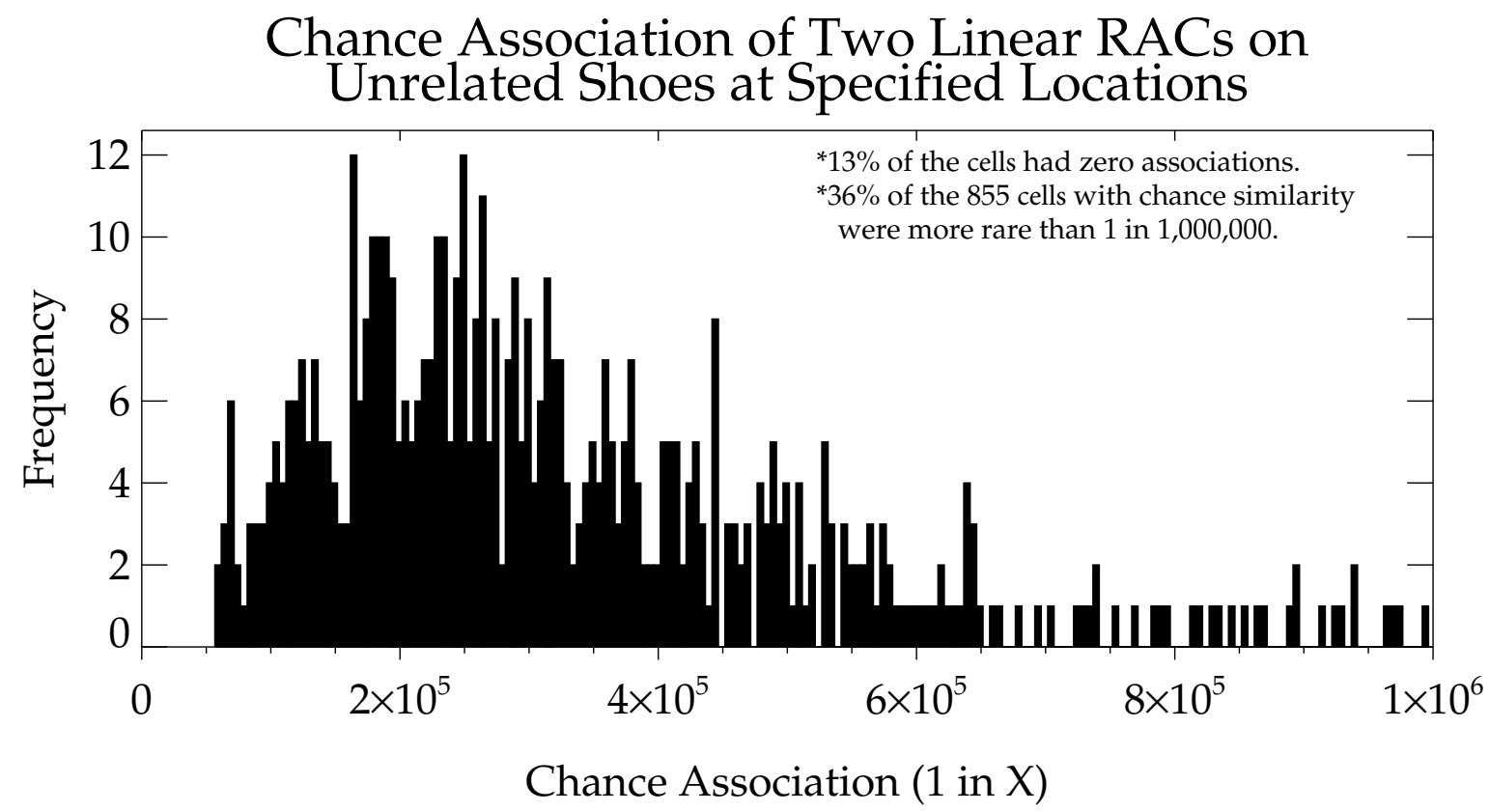

Figure 11: Frequency histogram for chance association of RACs with equivalent shape classifications based on the upper $95 \%$ credible interval. The plot also reports the number of cells with zero associations, and those with associations more rare than 1 in 1,000,000.

\section{Conclusions}

The purpose of this study was to estimate the probability of randomly selecting two unrelated shoes from a research database of 1,300 outsoles, and finding indistinguishable RACs at a specific location. Table 5 and Figs. 11 - 13 report summary statistics for these estimates, which provide insight into the range of possible chance associations that can result as a function of RACs belonging to different geometric categories and exhibiting different degrees of complexity. The results are a blend of empirical and modeled probabilities, based on a combination of subjective visual assessments, and objective mathematical scores, which the authors put forth as a reasonable compromise upon which to base forensic interpretation given the current state of the art within the pattern sciences. However, it is important to note five major factors associated with these estimates. First, the data is based on a random 


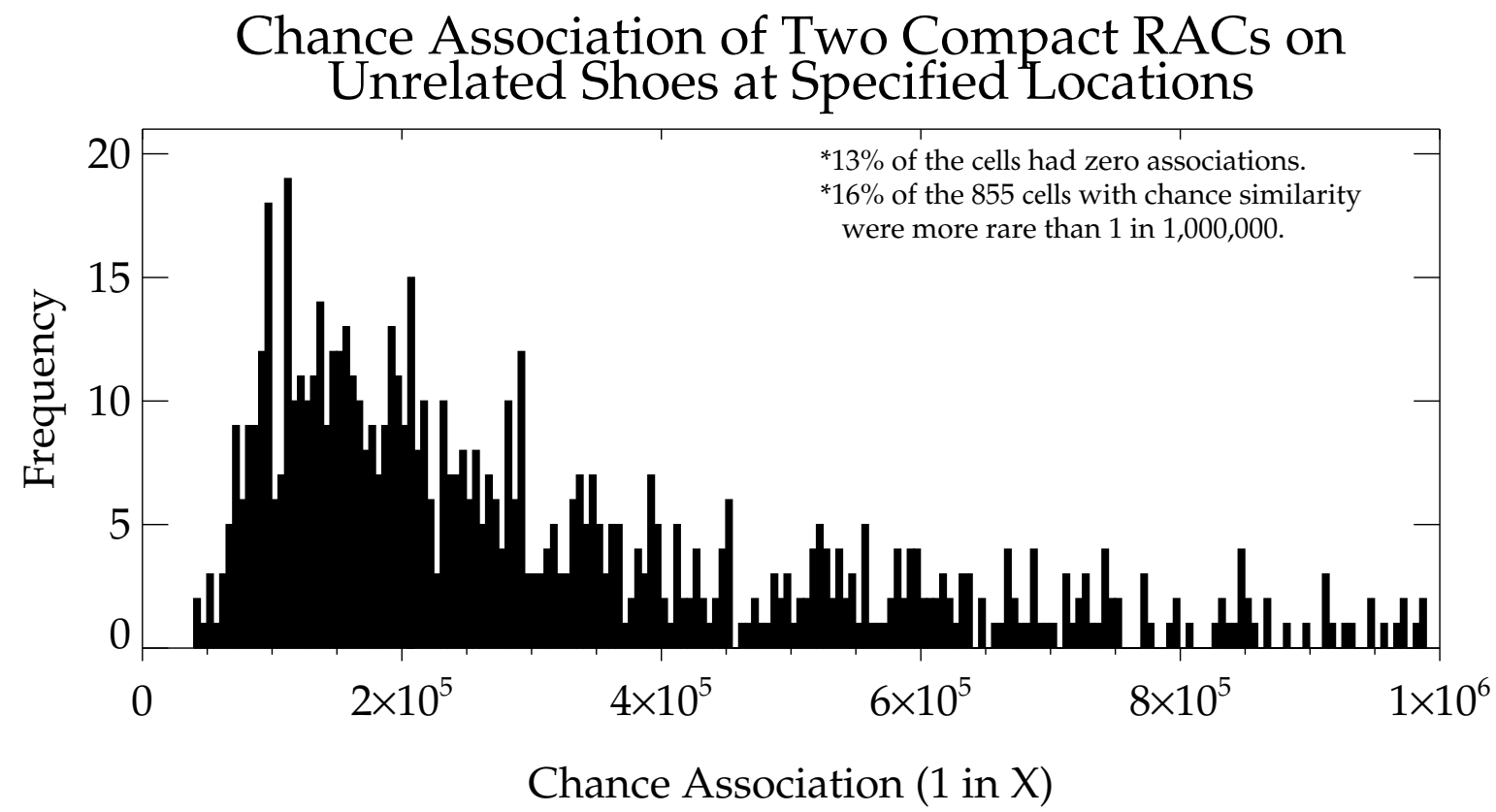

Figure 12: Frequency histogram for chance association of RACs with equivalent shape classifications based on the upper $95 \%$ credible interval. The plot also reports the number of cells with zero associations, and those with associations more rare than 1 in 1,000,000.

sampling of outsoles that vary in make, model and size, but are projected onto a normalized outsole [20]. Thus, it is inevitable that the process of position projection utilized in the normalization step, and the fact that class features are ignored, constrain the applicability of the estimates. Moreover, although the work accounts for tread in contact with the ground, the production of the tread element binary map is not a perfect reproduction of the actual outsole, and additional effort can be expended to increase the fidelity between the outsole and its representation as a tread element binary map.

Second, the estimates of indistinguishability are based on 91,607 subjective visual assessments of the most similar RACs per spatial cell. Although these estimates were repeated in duplicate by two analysts, and exhibit high inter-rater reliability (equal to or greater than 0.912), it should be noted that the analysts are researchers trained by practitioners, 


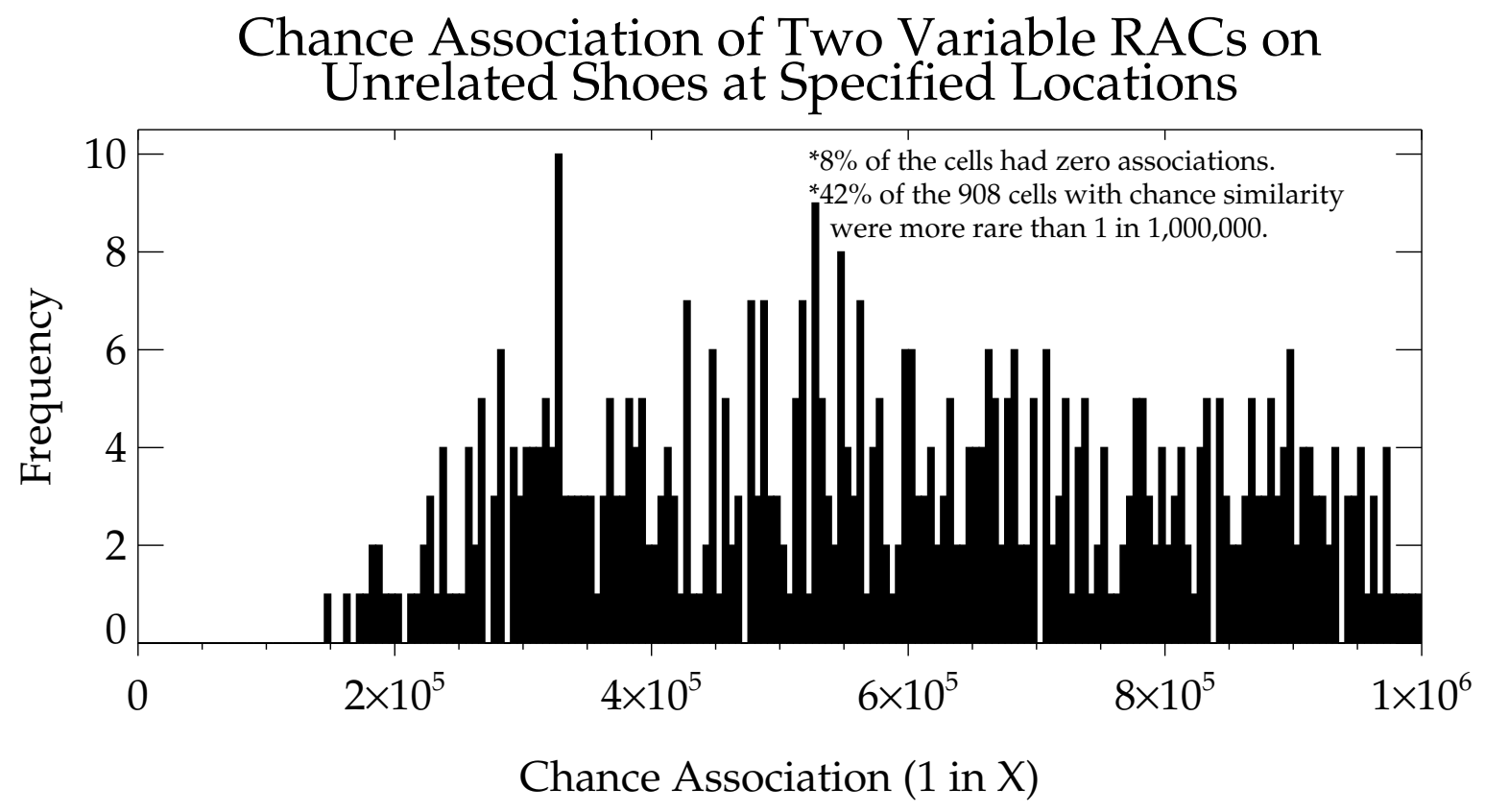

Figure 13: Frequency histogram for chance association of RACs with equivalent shape classifications based on the upper $95 \%$ credible interval. The plot also reports the number of cells with zero associations, and those with associations more rare than 1 in 1,000,000.

are not themselves practitioners, and it naturally follows that if different researchers and/or practitioners repeated this process, the results may exhibit variation. Third, the visual conclusions were used to generate 1,014,336 modeled predictions (the remaining RAC pairs in the database with positional association and equivalent shape classification). However, positional association to a $5 \mathrm{~mm} \times 5 \mathrm{~mm}$ spatial cell does not imply precise or exact overlaps in position, and the predictions are only as good as the model. Although the work uses statistical estimates to assert that the predicted and empirical estimates are statistically similar (or at least that the employed metrics failed to find a statistical difference), the model is crude, consisting of a simple polynomial regression based on \%A overlap. Thus, the model itself, and the process of reducing the similarity of complex geometric RACs into a single scalar value $(\% \mathrm{~A})$ is clearly very elementary in approach, and thus appropriate for additional 
inquiry. Fourth, the database used to generate the probabilities is of limited size, and no attempt has been made to estimate the power associated with this dataset. It is therefore unclear how the predictions might change if the dataset were to vary in size, and/or quality. In other words, it is important to keep in mind that the results are based on high quality test impressions, and therefore not indicative of crime-scene impressions that may be of more limited quality. Finally, the reported data should not be used to estimate the total chance association for increasing pairs of RACs (i.e., two pairs, three pairs, etc.) until the concept of spatial dependence is further vetted.

Despite these limitations, this work estimates the chance of randomly selecting two outsoles and finding forensically significant similarities in known non-matching RAC pairs, based on nearly a hundred-thousand visual comparisons performed in duplicate (which is several orders of magnitude larger than all past reports of empirical/visual comparison). Moreover, the results provide empirical evidence that can be used to validate and self-calibrate internalized and inferred knowledge of this chance. Moving forward, the same approach and dataset can be used to estimate random match probabilities for high quality RAC imagery, thereby providing examiners with research-based estimates of RAC rarity that can be used for validation and self-calibration of experience-based estimates of RAC rarity in casework. 


\section{Supplementary Materials}

The following describes updated shape categorization rules. Linearity and triangularity were previously defined in [20], orientation is the angle of either the major or minor axis $\left(\lambda_{1}\right.$ or $\left.\lambda_{2}\right)$ following eigendecomposition [20], RAC area $\left(R A C_{A}\right)$ is defined as the total number of pixels comprising the RAC in question, and RAC complexity is measured as the ratio of the RAC's actual area in pixels $\left(R A C_{A}\right)$, versus the number of pixels contained in the convex hull associated with the RAC in question $\left(R A C_{H}\right)$, according to equation Eq. 13 [33]:

$$
\text { Complexity }=\frac{R A C_{H}-R A C_{A}}{R A C_{H}}
$$

Based on the above definition, the following IF THEN ELSE intuitive classification rules were developed:

1. IF $($ area $\leq 10)$ THEN compact

2. $\mathrm{IF}($ area $\leq 25)$ AND $(\mid$ linearity $\mid \leq 25)$ THEN compact

3. IF (|linearity $\mid \leq 100)$ AND (complexity $\geq 0.6)$ THEN variable

4. IF (|linearity $\mid \geq 25$ ) AND (minor axis $\leq 1$ ) AND (complexity $\leq 0.6$ ) THEN linear

5. IF (|linearity $\mid \geq 100)$ AND (triangularity $\geq 0.8)$ THEN variable

6. IF $[(\mid$ linearity $\mid \geq 100)$ AND (minor axis $\leq 1.5)$ AND (triangularity $\leq 0.7)]$ AND $\left[(\right.$ complexity $\leq 0.6)$ OR $\left\{(\right.$ complexity $\leq 0.75)$ AND $\left(30^{\circ} \leq\right.$ orientation $\left.\left.\left.\leq 60^{\circ}\right)\right\}\right]$ THEN linear

7. IF $[(\mid$ linearity $\mid \geq 400)$ AND (minor axis $\leq 2)$ AND (triangularity $\leq 0.7)]$ AND $[($ complexity $\leq 0.6)$ OR $\left\{(\right.$ complexity $\leq 0.75)$ AND $\left(30^{\circ} \leq\right.$ orientation $\left.\left.\left.\leq 60^{\circ}\right)\right\}\right]$ THEN linear

8. IF $[(\mid$ linearity $\mid \geq 700)$ AND (minor axis $\leq 3)$ AND (triangularity $\leq 0.7)]$ AND $[($ complexity $\leq 0.6)$ OR $\left\{(\right.$ complexity $\leq 0.75)$ AND $\left(30^{\circ} \leq\right.$ orientation $\left.\left.\left.\leq 60^{\circ}\right)\right\}\right]$ THEN linear 
9. IF $[(\mid$ linearity $\mid \geq 1000)$ AND (minor axis $\leq 4)$ AND (triangularity $\leq 0.7)]$ AND $[($ complexity $\leq 0.6)$ OR $\left\{(\right.$ complexity $\leq 0.75)$ AND $\left(30^{\circ} \leq\right.$ orientation $\left.\left.\left.\leq 60^{\circ}\right)\right\}\right]$ THEN linear

10. IF (|linearity $\mid \geq 2000)$ AND (minor axis $\leq 4)$ THEN linear

11. IF (area $\leq 200)$ AND (|linearity $\mid \leq 25)$ AND (complexity $\leq 0.4)$ THEN compact 12. ELSE variable

\section{Reasons for Rules}

- Rule 1: Classify minute RACs (10 pixels $<0.5 \mathrm{~mm})$ as compact.

- Rule 2: Classify RACs with very small areas, excluding thin lines, as compact.

- Rule 3: Classify curvy elongated RACs as variable, rather than linear.

- Rule 4: Classify generally long and very thin RACs as linear.

- Rule 5: Classify elongated RACs with protrusions as variable, rather than linear.

- Rules 6-10: Classify generally long and thin RACs (with few deviations) as linear. This set of rules allows for RACs to become thicker as they get longer. In addition, the angle clauses allow for slightly higher complexity thresholds for diagonal lines because these are inherently more pixelated, and thus more complex, based on the way they were traced.

- Rule 11: Classify small RACs, with generally low elongation and complexity, as compact.

- Rule 12: Classify anything remaining as variable. 


\section{References}

[1] SWGTREAD, Guide for the examination of footwear and tire impression evidence; http://www.swgtread.org/images/documents/standards/published/08examination-final-03-2006.pdf (accessed February 2013) (2006).

[2] M. Cassidy, Footwear Identification (Reprint), Lightning Powder Company, Inc., 1995.

[3] Y. Shor, S. Wiesner, Methodological shift in scientifically evaluating shoeprint: A computerized system to statistically aid the expert reach the degree of certainty, Presented at International Association for Identification (IAI) Centennial Conference Sacramento, CA (2015).

[4] H. D. Sheets, S. Gross, G. Langenburg, P. J. Bush, M. A. Bush, Shape measurement tools in footwear analysis: A statistical investigation of accidental characteristics over time, Forensic Science International 232 (2013) 84-91.

[5] H. Wilson, Comparison of the individual characteristics in the outsoles of thirty-nine pairs of Adidas Supernova Classic shoes, Journal of Forensic Identification 62(3) (2012) $194-203$.

[6] J. Skerrett, C. Neumann, I. Mateos-Garcia, A Bayesian approach for interpreting shoemark evidence in forensic casework: Accounting for wear features, Forensic Science International 210 (2011) 26-30.

[7] N. Petraco, C. Gambino, T. Kubic, D. Olivio, N. Petraco, Statistical discrimination of footwear: A method for the comparison of accidentals on shoe outsoles inspired by facial recognition techniques, Journal of Forensic Sciences 55 (2010) 34-41.

[8] T. Hannigan, L. Fleury, R. Reilly, B. O’Mullane, P. de Chazal, Survey of 1276 shoeprint 
impressions and development of an automatic shoeprint pattern matching facility, Science \& Justice 46 (2006) 79-89.

[9] R. Stone, Footwear examination: Mathematical probabilities of theoretical individual characteristics, Journal of Forensic Identification 56 (2006) 577-599.

[10] C. Champod, R. Voisard, A. Girod, A statistical study of air bubbles on athletic shoe soles, Forensic Science International 109 (2000) 105-123.

[11] R. Davis, A. Keeley, Feathering of footwear, Science \& Justice 40 (2000) 273-276.

[12] R. Davis, J. DeHaan, A survey of men's footwear, Journal of the Forensic Science Society 17 (1977) 271-285.

[13] A. Fawcett, The role of the footmark examiner, Journal of the Forensic Science Society 10 (1970) 227-244.

[14] T. W. Adair, J. LeMay, A. McDonald, R. Shaw, R. Tewes, The Mount Bierstadt study: An experiment in unique damage formation in footwear, Journal of Forensic Identification 57(2) (2007) 199-205.

[15] C. Hamburg, R. Banks, Evaluation of the random nature of acquired marks on footwear outsoles, in: 2010 Impression and Pattern Evidence Symposium, Clearwater, Florida, 2010.

[16] M. Marvin, Oral presentation: A look at close non-match footwear examinations, International Association for Identification (IAI) Centennial Conference, August 2015.

[17] Y. Yekutieli, Y. Shor, S. Wiesner, T. Tsach, Expert Assisting Computerized System for Evaluating the Degree of Certainty in 2D Shoeprints, Technical Report, National 
Institute of Justice, U.S. Department of Justice, Office of Justice Programs Technical Report, 2016.

[18] M. Khalil, Assessing the degree of similarity between accidental patterns on outsoles associated with shared and independent activities, Masters Thesis, Cedar Crest College (2013) 1-56.

[19] N. Damary, M. Mandel, S. Wiesner, Y. Yekutieli, Y. Shor, C. Spiegelman, Dependence among randomly acquired characteristics on shoeprints and their features, Forensic Science International 283 (2018) 173-179.

[20] J. Speir, N. Richetelli, M. Fagert, M. Hite, W. J. Bodziak, Technical note: Quantifying randomly acquired characteristics on outsoles in terms of shape and position, Forensic Science International 266 (2016) 399-411.

[21] T. Wallace, O. Mitchell, Analysis of three-dimensional movement using Fourier descriptors, IEEE Transactions on Pattern Analysis and Machine Intelligence 2(6) (1980) $583-588$.

[22] I. Bartolini, P. Ciaccia, M. Patella, WARP: Accurate retrieval of shape using phase of Fourier descriptors and time warping distance, IEEE Transactions on Pattern Analysis and Machine Intelligence 27(1) (2005) 142-147.

[23] C. Dalitz, C. Brandt, S. Goebbels, D. Kolanus, Fourier descriptors for broken shapes, EURASIP Journal on Advances in Signal Processing 161 (2013) 1-11.

[24] N. Richetelli, M. Nobel, W. Bodziak, J. Speir, Quantitative assessment of similarity between randomly acquired characteristics on high quality exemplars and crime scene impressions via analysis of feature size and shape, Forensic Science International 270 (2017) 211-222. 
[25] J. Speir, A Quantitative Assessment of Shoeprint Accidental Patterns with Implications Regarding Similarity, Frequency and Chance Association of Features, Technical Report NCJ 251522, National Criminal Justice Reference System, https://www.ncjrs.gov/App/Publications/abstract.aspx?ID=273702, 2018.

[26] E. DeLong, D. DeLong, D. Clarke-Pearson, Comparing the area under two or more correlated receiver operating characteristic curves: a nonparametric approach., Biometrics $44(3)(1988) 837-845$.

[27] D. Huttenlocher, G. Klanderman, W. Rucklidge, Comparing images using the Hausdorff distance, IEEE Transactions on Pattern Analysis and Machine Intelligence 15(9) (1993) $850-863$.

[28] K. Gwet, Handbook of Inter-Rater Reliability, Four Edition, The Definitive Guide to Measuring the Extent of Agreement Among Raters, Advanced Analytics, LLC, 2014.

[29] Y. Shor, S. Wiesner, T. Tsach, R. Gurel, Y. Yekutieli, Inherent variation in multiple shoe-sole test impressions, Forensic Science International 285 (2018) 189-203.

[30] F. Korner-Nievergelt, T. Roth, S. von Felten, J. Guélat, B. Almasi, P. Korner-Nievergelt, Bayesian Data Analysis in Ecology Using Linear Models with R, BUGS, and Stan, Elsevier Academic Press, 2015.

[31] E.-J. Wagenmakers, F. S., Notes and comments: AIC model selection using Akaike weights, Psychonomic Bulletin \& Review 11 (1) (2004) 192-196.

[32] D. Hosmer, S. Lemeshow, R. Sturdivant, Applied Logistic Regression, Third Edition, John Wiley \& Sons, 2013.

[33] T. Brinkhoff, H. Kriegel, R. Schneider, A. Braun, Measuring the complexity of polygonal objects, ACM-GIS (1995). 


\section{Evaluation of Spatial Randomness}




\section{Evaluation of a Large-Scale Database of Randomly Acquired Characteristics from Shoe Outsoles: Analysis of Spatial Randomness}

Keywords: Forensic Footwear, Randomly Acquired Characteristics, Spatial Distribution, Spatial Randomness, Spatial Autocorrelation

\section{Introduction}

Analysis and comparison of questioned footwear impressions with known shoes can reveal links between a suspect and a crime scene and aid in reconstruction of a series of events surrounding a crime. The strength of this linkage is directly affected by the class features, wear patterns, and randomly acquired characteristics (RACs) that are both present on an outsole and reliably transferred to an impression [1]. Although class characteristics and wear have been shown to be immensely useful for narrowing down the pool of suspect shoes that could have made a given crime scene impression, these features alone preclude a more certain conclusion (i.e., high degree of association or identification) because they can be shared by a number of other shoes [2-7]. As such, the final step of forensic footwear analysis, and arguably the most difficult, lies in the detection and comparison of randomly acquired characteristics [8]. These features are developed through shoe use and can result in either removal (in the form of scratches, tears, holes, etc.) or addition (in the form of gum, nails, etc.) of material to the surface of an outsole [9]. Furthermore, the immense variation in feature location, size, general shape, orientation, and perimeter morphology are what form the basis of the ever-increasing discriminating power that can be achieved when comparing RACs on known and questioned impressions. Consequently, an agreement of class characteristics, wear, and a number of RACs, in addition to an assessment of rarity and sufficiency of these features, is required in order for an examiner to reach an opinion of source association for a crime scene impression $[8,9]$. 
"Randomly" Acquired Characteristics

Forensic footwear evidence comparisons use RACs as the basis of source identification due to their strong discriminating potential, which is underpinned by a multitude of factors relating to their development and resulting appearance on an outsole and within an evidence impression. First, the acquisition of an accidental characteristic is caused by the addition of a substance (gums, nails, etc.) or removal of material (nicks, scratches, etc.); the unpredictable nature of this acquisition results from a number of variables impacting outsole wear, including the outsole material composition, tread pattern, surface of contact, pressure of contact, and gait of the wearer [9]. Once a RAC develops and can be readily observed on the shoe, its appearance can offer significant differentiating power owing to the multitude of possible shapes, sizes, and orientations that the feature can assume [9]. Ultimately, the weight of RACs for informing source identification (or exclusion) for footwear evidence is dependent upon the fidelity of the transfer from the outsole to a crime scene impression, which is related to a variety of deposition factors, such as the medium, substrate, pressure, and torque [9]. Therefore, given the presumed stochastic nature of development, a major assumption in the field of forensic footwear is that accidental features exhibit spatial "randomness." In fact, this assumption is so entrenched that the moniker of "random" has even become part of the label attributed to these features (i.e., "randomly acquired characteristics"). As a result, several studies have been conducted to ascertain the chance of correspondence between features on unrelated sources [10-15]. The collective result from these previous research efforts validate the aforementioned assertion, that RACs are highly discriminating. More specifically, even when RACs appear in the same location and of relatively the same shape, their sizes, orientations, and/or perimeter morphologies can be used to distinguish between features [10-15].

A natural extension of this research is to seek out a quantitative probability estimate of chance association, and within the forensic community, an estimate of random match probability. In fact, several studies have been conducted that sought to characterize this probability, utilizing both theoretical and empirical data [16-18]. While the approaches (binomial probability, combination of probabilities) and the characteristics of interest (RAC location, size, shape, and/or orientation) for each of these studies varied, all three made 
the same assumption: that accidentals are random and therefore their acquisition can be considered independent [16-18]. In accepting this assumption, the probability of observing coincidental features was ascribed to be the multiplication of the probability of the presence of each individual RAC. However, should the distribution of features be dependent (the presence of one feature either promotes or prevents the presence of another feature), then the probability of each feature cannot be multiplied together and must be reconsidered to include this interaction. In order to conduct valid statistical analyses, therefore, it is important to first characterize the spatial distribution of accidentals.

Despite the fact that there is a large body of previous research seeking to support the discriminating potential of RACs due to their randomly acquired nature, and subsequent works that utilize this assumption to quantitatively describe the power of accidentals, there has yet to be an actual statistical test of this randomness. More specifically, if RACs are acquired randomly then it is expected that their spatial distribution on an outsole will be random as well. Furthermore, an examination of the degree of randomness of RACs would help to direct future statistical analysis of the chance co-occurrence of features which would in turn offer additional quantitative support to the use of acquired characteristics for footwear source attributions. In order to achieve this goal, the locations of RACs on a given outsole will be regarded as spatial events (much like trees within a given forest) comprising a spatial point pattern, which is "a set of locations, irregularly distributed within a designated region and presumed to have been generated by some form of stochastic mechanism" [19]. The resulting spatial point pattern will be used to evaluate the adherence to randomness of RAC distributions through application of common spatial statistics methods. In doing so, this work provides foundational research that will serve as the basis upon which further statistical exploration/description of these features can occur (e.g., whether multiplication of independent probabilities can be conducted or if more sophisticated methods must be utilized to account for dependence).

\section{Overview of Complete Spatial Randomness (CSR)}

Spatial statistics are used extensively in numerous fields, including biology, ecology, and epidemiology, to evaluate the spatial arrangement of features, samples, and/or attributes (be 
they species of plants, animals, or viruses). More specifically, these techniques evaluate the presumed stochastic distribution of a set of points across a Euclidean plane [19, 20]. The cornerstone of spatial statistics is the evaluation of complete spatial randomness (CSR), the condition under which no discernible pattern exists in the spatial arrangement of features or events [19-21]. Assessment of spatial point patterns typically begins with an analysis of the extent to which a given dataset conforms to CSR in order to determine the direction of analysis thereafter. For example, if statistical analysis fails to detect a pattern, and thus fails to disprove that the pattern is not random, no further statistical analysis is generally warranted [19]. On the other hand, if indications of clustering are revealed by the CSR tests, then further examination via cluster analysis may provide information about the factors that are driving the spatial arrangement of features.

As applied to forensic footwear, randomly acquired characteristics (RACs) can be considered a spatial point pattern of features acquired through the stochastic mechanism of wear. Individually, each accidental serves as an "event" and its spatial location on the outsole can be recorded. This information can then be utilized to determine the degree to which the distribution of the markings can be considered random, an important assumption made by footwear experts when reaching conclusions about the potential source of a crime scene impression. Nonetheless, a statistical evaluation of a single shoe does little to offer evidence as to the true "randomness" of RACs. Rather, a database of features is necessary in order to determine the degree to which accidentals, in general, can be considered remnants of random events, which is the aim of the proposed research: to determine the degree to which accidentals conform to CSR using a variety of complementary techniques.

\section{Testing for Complete Spatial Randomness}

In order for a spatial point pattern to conform to complete spatial randomness, it must satisfy two main criteria [21]. First, the points must exhibit homogeneity in which points do not have a preference for any given location. In terms of footwear evidence, this means that any location on the outsole is equally likely to acquire accidentals. In practicality, this concept must be updated to account for the fact that footwear can be specifically designed such that not all areas of the outsole are in contact with the ground (e.g., raised 
insteps, outsoles with tread/lug patterns, etc.). Additionally, the events must be considered independent and as such, the information about the outcome at a given location does not affect the outcome in another region [21]. More specifically, this means that the development of a RAC at a specific location does not promote or deter the development of a separate feature in another area. In summary, events within a spatially random distribution are equally likely to occur anywhere within the study area and do not impact the spatial location of neighbors [19-21].

In an effort to apply spatial statistics to forensic footwear evidence, the proposed research will employ a variety of different methods to evaluate the degree of spatial randomness of RACs across an outsole database. This multiphasic testing approach is considered beneficial since the proposed tests are complimentary in nature. More specifically, three distinct categories of techniques for assessing CSR will be utilized for this study: quadrat, distance, and autocorrelation approaches. [19, 20, 22].

\section{Materials and Methods}

\section{Database and Pre-Processing}

The Speir Research Group at West Virginia University (WVU) houses a collection of 1,300 shoes for analysis, varying in both class and subclass characteristics (manufacturer, style, size, degree of wear, etc.), but is largely comprised of athletic styles (approximately $86 \%$, or 1122 shoes) [14]. Each of the aforementioned outsoles were examined for the presence of accidentals, and in total 72,306 RACs were identified, characterized, and localized via a semi-automated image processing chain [14]. Subsequently, RACs were classified based on shape and remapped onto a standard outsole using normalized polar coordinates $\left(r_{n o r m}\right.$, $\theta$ ), and finally segmented into 987 bins to create a frequency heatmap (as detailed in [14, 15]). This heatmap is publicly available at http://www.4n6chemometrics.com/database/ and provides information about RAC frequencies, number of shoes contributing RACs, and probabilities of encountering RACs at each location in the database $[14,15]$.

As previously noted, the database contains a variety of shoes with different class and subclass characteristics. A consequence of this is considerable variation in outsole geometric design. Since this research is focused on RACs that result from the removal of material 
from the outsole (nicks, cuts, scratches, etc.) and their appearance in two-dimensional impressions, binary contact maps describing tread contact with the ground were required. To obtain these maps, each impression was poly-warped to fit the approximate shape and size of the standardized heatmap outsole, thereby allowing for comparison with the previously normalized RAC locations across all shoes. Next, a tread element binary mask was automatically extracted using a series of morphological operators [15], where black pixels represent tread elements in contact with the ground and white pixels indicate recessed areas. Lastly, the number of "on" pixels (black) were likewise counted within each of the heatmap cells and summed across shoes to obtain a total number of contact pixels as well as the total number of shoes with contact in a given region (a binary decision based on criteria outlined in [15]).

\section{Point Process Simulation}

The primary goal of this research was to understand and describe the spatial distribution of empirically observed RACs in the WVU outsole database. In pursuit of this objective, a set of simulations were created for each shoe with known underlying patterns (or lack thereof), using the observed number of RACs identified on each shoe as the number of points to simulate and the contact-area modified binary maps as a "window" to describe the positions on which these points could fall [21].

Given that one of the most fundamental analyses in spatial statistics is an evaluation of CSR, the first group of simulations required were Poisson random simulations intended to serve as null distributions for this purpose. A minimum of 1,000 Poisson random realizations per shoe were created using the following methodology [21]:

1. Determine number of RACs $(n)$ on shoe and compute shoe-specific intensity $\left(\lambda=n / A_{c}\right.$, where $A_{c}$ represents the number of "on" pixels in the shoe's binary contact map).

2. Simulate a Poisson random point process with the given intensity onto the shoe-specific binary contact map.

3. Retain Poisson simulation if the number of simulated points $\left(n_{s}\right)$ equals the number of observed RACs $(n)$, otherwise repeat Step 2. 
Following simulation of the null distributions, two sets of alternative distributions (clustered and dispersed) were created in order to characterize any departures from randomness and evaluate the power of traditional tests of spatial randomness as applied to footwear features. In an effort to determine the existence of patterns in the empirical data at various ranges, each alternative was simulated on two different scales: i.) heatmap bin size, and ii.) mean lug size. The heatmap bins were previously defined as $5 \mathrm{~mm} \times 5 \mathrm{~mm}$ in size $(\sim 120 \mathrm{px}$ $\times 120 \mathrm{px}$ at a resolution of 600ppi), yielding an approximate diagonal diameter of 170px (and thus a radius of $85 \mathrm{px}$ ) which was used to inform size parameters for the alternative simulations on the bin scale. Subsequently, to determine mean lug size, 130 lugs were measured in duplicate on randomly selected shoes (excluding those outsoles without clear lugs) to determine the range of tread element sizes in the database. The resulting mean diameter was $399 \mathrm{px} \pm 195 \mathrm{px}$ (or approximately $17 \mathrm{~mm} \pm 8 \mathrm{~mm}$ ), which likewise was used to inform simulations moving forward. Furthermore, for clustered simulations, cluster sizes must be specified [21]; as such, the maximum number of RACs per bin per shoe was investigated and this revealed a mean of 4 RACs \pm 2 RACs.

First, the clustered alternative distributions were generated. When points are located closer together in space than would be expected under randomness (i.e., points have an attractive relationship), a clustered pattern likely exists in the data [20, 21]. A minimum of 500 clustered realizations per shoe, at each of two different scales (clustered within heatmap bins and within outsole lugs) were created using the following methodology [21]:

1. Determine number of RACs $(n)$ on shoe and compute shoe-specific intensity $\left(\lambda=n / A_{c}\right.$, where $A_{c}$ represents the number of "on" pixels in the shoe's binary contact map).

2. Randomly assign cluster size $\left(n_{c}\right.$ between $\left.2-6\right)$ and computer cluster-modified intensity accordingly $\left(\lambda_{c}=\lambda / n_{c}\right)$.

3. Simulate random parent points to represent cluster locations according to clustermodified intensity $\left(\lambda_{c}\right)$.

4. Center a uniform disc of radius $r_{c}$ on each parent point (where $r_{c}=85 \mathrm{px}(3.5 \mathrm{~mm})$ for "in bin" clustering or a randomly sampled value from $r_{c}=100,105,110, \ldots, 300 \mathrm{px}(4.2$ - 12.5mm) for "on lug"). 
5. Remove each parent point and simulate $n_{c}$ points (a randomly sampled value from $\left.n_{c}=2,3,4,5,6\right)$ within each disc and on contact pixels.

6. Retain clustered simulation if $n_{s}$ (number of simulated RAC locations) $=n$ (number of empirical RAC locations), otherwise repeat Steps 2-4.

Lastly, the dispersed pattern realizations were created, wherein this type of structure results in points being spread further apart than would be expected under randomness (i.e., points have a repulsive relationship) [20,21]. Similar to the clustered simulations, the goal was to create two sets of realizations for the dispersed points on the heatmap bin and shoe lug scales. A minimum of 500 dispersed realizations per shoe, at each of two different scales (dispersed outside of heatmap bins and outside of outsole lugs, when allowable) were created using the following methodology [21]:

1. Determine number of RACs $(n)$ on shoe and set this equal to the number of points to be simulated $\left(n_{s}\right)$.

2. Specify inhibition distance $r_{i}$ (where $r_{i}=85 \mathrm{px}(3.5 \mathrm{~mm})$ for "out of bin" or maximum allowable between 400-75px (16.7 - 3.1mm) for "outside lug" dispersion (see below for additional detail)).

3. Simulate uniform points within the binary contact map one-by-one.

4. For each point simulated, retain if it is further than $r_{i}$ distance of a pre-existing point.

5. Repeat Steps 3-4 until no additional points can be added.

6. Retain dispersed simulation if $n_{s}=n$, otherwise repeat Steps 3-5.

Depending on the degree of contact and the number of RACs identified on a given outsole, points can only be dispersed so much before they run out of space. Hence, in order to conduct the "outside lug" simulations for dispersion it was necessary to determine the maximum allowable dispersion factor for each shoe, which was then used as the parameter in Step 2 above. For each shoe, $r_{s}$ was initially set to $400 \mathrm{px}(16.7 \mathrm{~mm})$ (or the mean radius of lugs) and the above process was completed. Given that each simulation can create a different number 
of points, depending on the starting point and the order of subsequently thrown points, this process was completed 25 times. If $n_{s} \neq n$ for all of the 25 trials, then $r_{s}=r_{s}-25$ (or $375 \mathrm{px}$ $(15.6 \mathrm{~mm})$ for the first step down) and the steps were repeated until a successful $\left(n_{s}=n\right)$ simulation was created, at which time this value of $r_{s}$ was retained as the maximum allowable dispersion factor for the given shoe and used moving forward.

After creation of all shoe-specific simulations, 10,000 random heatmap realizations were compiled for each of the contact-modified distributions by randomly selecting a point process from each shoe and combining all data to represent 72,306 simulated RACs under the given distribution. These "RACs" were then localized and binned in the same manner as conducted for the empirical dataset, so for each theoretical heatmap the simulated RAC locations were available as point coordinates (both in Cartesian and polar space) as well as bin memberships.

\section{Poisson Simulation Proof of Concept: Flat Outsole Testing}

As previously discussed, in order for a point process to fall under complete spatial randomness two criteria must be satisfied: i.) points can fall anywhere within the sample space (i.e., homogeneous), and ii.) points cannot be influenced by other points (i.e., independent) [19-21]. In terms of shoe outsoles, the first assumption is challenged because the vast majority of shoes (especially in the database under study) do not have uniform, flat outsoles, and instead have recessed areas between tread elements that are not in contact with the ground to develop a RAC. As such, traditional methods for testing adherence to CSR using theoretical underlying distributions would not be appropriate.

However, it is prudent to provide evidence that the simulation procedure used in this study to create Poisson random distributions (albeit on contact-modified outsole imagery) actually abides by CSR. To achieve this, 2,000 flat outsole simulations were created, wherein 72,306 "RACs" were placed within the standard outsole frame (which was used as a uniform window for this phase) and then localized using the same procedure as the heatmap detailed earlier. Then, these random heatmap realizations were tested for adherence to CSR using quadrat (chi-square goodness of fit and paired quadrat analysis) and distance (average nearest neighbor and Pollard's distance) methods. 
Quadrat-Based Methods

Quadrats are a collection of small sub-areas within a larger study region that are defined by their coverage area, the total number of events each encapsulates, or both [20]. Using this definition, quadrat-based methods attempt to evaluate changes in point density. Overall, quadrat-based analysis methods are easy to implement, allowing for quick evaluation of global spatial randomness and are historically one of the most common preliminary methods for CSR analysis [19-21].

\section{Chi-Square Goodness of Fit Approach}

One of the most simple of the quadrat-based methods is the chi-square goodness of fit approach (GOF), which entails comparing an empirical dataset of spatial events (binned into quadrats) to the expected number of events over the same area, within the same number of quadrats $[21,23,24]$. The number of expected RACs $\left(e_{q}\right)$ per bin $q(q=1,2, \ldots, Q=987)$ was computed according to Eq. 1 [21, 24], where $N$ represents the total number of points $\left(72,306\right.$ in this case) and $a_{q}$ represents the number of pixels comprising the bin (maximum of 14,161 for full bins).

$$
e_{q}=N \times \frac{a_{q}}{\sum_{q=1}^{Q} a_{q}}
$$

The Poisson simulated RAC counts per bin from the flat outsole realizations $\left(n_{q}\right)$ were then compared to the calculated expected counts per bin using a chi-square test, with 986 degrees of freedom $(d f=Q-1)$, and computing the test statistic according to Eq. 2 .

$$
\chi^{2}=\sum_{q=1}^{Q} \frac{\left(n_{q}-e_{q}\right)^{2}}{e_{q}}
$$

The computed test statistics were compared against the chi-square critical value at a significance level of $\alpha=0.05$ to determine if there was evidence for deviation from randomness (reject the null hypothesis) for each of the 2,000 flat outsole simulations and the associated $p$-values were retained. 


\section{Paired Quadrat Approach}

Rather than simply considering the global frequency of quadrat counts as in the chisquare GOF test, the paired quadrat approach assesses differences in RAC presence at a local scale by evaluating differences in RAC counts between randomly mated quadrats. More specifically, this method determines the variation in stochastically paired quadrats separated by fixed (but multiple) distances, and therefore requires quadrats to be randomly paired before computation. The following steps were utilized to pre-define the mates [25]:

1. Set quadrat dimensions, $w \times l(e . g ., w=l=5 m m)$.

2. Define centroid distances $\left(h_{j}=h_{1}, h_{2}, \ldots, h_{J}\right)$ based on quadrat half-size for $j=$ $1,2, \ldots, J$. For this study, quadrats $2,3,4$, and 5 neighbors away (equivalent to distances between centroids ranging from approximately 250px to 600px (10.4-25.0mm)) in order to encompass roughly the mean lug diameter $+/$ - one standard deviation.

3. Randomly sample $N=n_{h} \times J$ quadrat pairs separated by distance $h_{j}$. For this study, $n_{h}=50$ for a total of 200 unique pairings (note that there is a limit in the number of pairs that can be selected at higher distance orders).

i. Randomly select a quadrat $(q)$;

ii. Find all candidate pairs $\left(s_{1}, \ldots, s_{x}\right)$ separated by $h_{j}$ from $q$;

iii. Randomly select a candidate from $s_{1}, \ldots, s_{x}$;

iv. Repeat steps (i.) - (iii.) for all $h_{j}=h_{1}, \ldots, h_{J}$, sampling without replacement;

v. Repeat steps (i.) - (iv.), continuing to sample without replacement until each paired distance $\left(h_{j}=h_{1}, \ldots, h_{J}\right)$ has $n_{h}$ paired quadrats.

For $h_{j}=h_{1}, \ldots, h_{J}$, the mean squared difference $\left(V_{h j}\right)$ was computed according to Eq. 3 $[20,26]$, where $q_{k}$ and $s_{k}$ represent the number of events in a given pair of quadrats $(k)$ for all randomly selected quadrat pairs, and $k=1,2, \ldots n_{h}$, separated by $h_{j}$ units.

$$
V_{h j}=\frac{1}{2 n_{h}} \sum_{\substack{k=1 \\ 59}}^{n_{h}}\left(t_{k}-s_{k}\right)^{2}
$$


It is expected that for a random point pattern, the variance $\left(V_{h j}\right)$ will be approximately constant across all distance classes [20]. Therefore, Bartlett's test for equal variances was used to evalute adherence to CSR according to Eq. 4 [20, 25, 27]. The variances were considered unequal if $T>\chi_{1-\alpha, J-1}^{2}$, where $\chi_{1-\alpha, J-1}^{2}$ is the critical value of the chi-square distribution with $J-1$ degrees of freedom.

$$
\begin{aligned}
T & =\frac{(N-J) \ln V_{p}-\sum_{j=1}^{J}\left(n_{h}-1\right) \ln V_{h j}}{1+\frac{1}{3(J-1)}\left(\sum_{j=1}^{J} \frac{1}{n_{h}-1}-\frac{1}{N-J}\right)} \\
V_{p} & =\sum_{j=1}^{J} \frac{\left(n_{h}-1\right) V_{h j}}{(N-J)}
\end{aligned}
$$

\section{Distance-Based Methods}

Tests for complete spatial randomness based on distances use precise measurements of locations, rather than generalized cells, and therefore offer the advantage of detecting smaller deviations from CSR as compared to quadrat-based techniques [20]. Numerous test statistics for evaluating CSR as a function of distance exist (see Cressie, 2015 [20] for a detailed description of these methods), however, these generally fall into two major categories: those that measure distance between neighboring events (RACs in this case), and those that measure distance between an event and a random sample point [20]. The distance evaluations are then transformed into a test statistic and compared to that derived from a corresponding theoretical distribution to determine adherence to the null hypothesis (or complete spatial randomness) [20, 21, 24, 28, 29].

\section{RAC to RAC Method: Average Nearest Neighbor}

One of the most commonly used and simple to implement tests for detecting departures from CSR using distances between points is the average nearest neighbor (ANN) approach [24, 29-31]. Similar to the chi-square GOF test, this method compares observed mean distances between neighboring events (RACs in this case) to a computed expected value. When determining the expected value for ANN, it is important to adjust for edge effects, which arise because the test statistic assumes an unbounded spatial region, which in practicality ac- 
tually has a boundary (e.g., the frame of the standard heatmap outsole). The edge-corrected expected value $\left(\bar{D}_{e}\right)$ is thus calculated according to Eq. 5 [31], where $N$ is the number of observations $(72,306 \mathrm{RACs})$ and $P$ and $A$ are the perimeter $(8,598 \mathrm{px}$ or $358 \mathrm{~mm})$ and area $\left(12,748,081 \mathrm{px}\right.$ or $\left.\sim 21,235 \mathrm{~mm}^{2}\right)$ of the standard heatmap outsole window, respectively. Note that the subsequent additions to the initial term represent the edge adjustment previously determined through simulation by Donnelly (1978) [32].

$$
\bar{D}_{e}=0.5 \sqrt{\frac{A}{N}}+0.0514 \sqrt{\frac{P}{N}}+\left(\frac{0.041}{\sqrt{N}} \times \frac{P}{N}\right)
$$

After obtaining the theoretical expected value, the average nearest neighbor distance for each of the 2,000 flat outsole Poisson simulations was determined $\left(\bar{D}_{o}\right)$ and subsequently transformed to a Z-score according to Eq. 6, where $i$ represents a simulated RAC of interest $(i=1,2, \ldots, N)$ and $d_{i}$ represents the Euclidean distance between $i$ and its nearest neighboring RAC.

$$
\begin{aligned}
\bar{D}_{o} & =\frac{\sum_{j=1}^{N} d_{i}}{N} \\
S E_{A N N} & =\frac{0.26136}{\sqrt{\frac{N^{2}}{A}}} \\
Z & =\frac{\bar{D}_{o}-\bar{D}_{e}}{S E}
\end{aligned}
$$

The average nearest neighbor statistic follows an approximately Normal distribution $\left(N\left(\bar{D}_{o}, S E_{A N N}\right)\right)$ under the null hypothesis of randomness; therefore, the computed test statistic $(Z)$ was compared to the critical value for the given distribution at $\alpha=0.05$ in order to determine whether evidence exists to suggest departures from CSR for each of the 2,000 Poisson simulations.

\section{Point to RAC Method: Pollard's Distance Statistic}

Unlike the average nearest neighbor approach, Pollard's statistic $(P o)$ utilizes the distance between synthetic random points $(i)$ and the $j$ th-nearest $\mathrm{RAC}(\mathrm{s})$ to evaluate the spatial 
randomness of features [20, 28, 33]. Furthermore, Pollard's statistic is sensitive for detecting departures from CSR at higher distance orders (i.e., for RACs further apart) and as such can be used to test for randomness at various scales [28]. In order to compute Pollard's statistic for the 2,000 flat outsole simulations, the following procedure was employed, where $j$ represents the distance order (e.g., the nearest neighbor is $j=1$ ) and $x_{i j}$ represents the distance from the $i$ th synthetic point to its $j$ th RAC neighbor [28]:

1. Randomly project $n=100$ synthetic points onto the standard heatmap outsole.

2. In order to adjust for edge effects, an edge correction procedure was incorporated as follows. For each synthetic point $(i)$, find its 5th nearest neighbor (simulated RAC $\left.\left(x_{i 5}\right)\right)$ as well as the distance from the point to the perimeter of the study region $\left(x_{i p}\right)$. If $x_{i p} \leq x_{i 5}$, then delete this point and re-project a new point, again checking $x_{i 5}$ versus $x_{i p}$.

3. Compute Pollard's test statistic $(P o)$ for $j=1$, according to Eq. 7 .

$$
P o=12 j^{2} n \frac{\left[n \ln \left(\sum_{i=1}^{n} \frac{x_{i j}^{2}}{n}\right)-\sum_{i=1}^{n} \ln \left(x_{i j}^{2}\right)\right]}{[(6 j n+n+1)(n-1)]}
$$

4. Repeat steps 1-3 a total of 1,000 times.

5. Repeat steps 1-4 for each distance order $(j=2,3,4,5)$.

Under CSR, $(n-1) P_{o}$ follows an approximately chi-squared distribution with degrees of freedom $(d f=n-1)$; using this information, the upper and lower limits of the $95 \%$ confidence interval on the $\chi^{2}$ distribution were obtained. Subsequently, $(n-1) P_{o}$ was compared to this interval to evaluate the null hypothesis, that the data conforms to complete spatial randomness $[20,28]$.

\section{Power Testing of Traditional CSR Tests on Contact-Area Modified Simulations}

Although flat outsole simulations were used as a baseline to obtain evidence that the employed methodology for simulating Poisson point processes do in fact adhere to spatial randomness, all subsequent data analysis was conducted on contact-modified data in order 
to accurately represent the true state of the spatial area. More specifically, given that many outsoles have tread elements with intervening recessed areas, and considering that the WVU database is constructed of two-dimensional impressions only, there are likely areas on each outsole where RACs cannot exist. Thus, it was imperative to account for these non-contact areas in all further examinations.

As an initial step in characterizing the distribution of RACs on outsoles, traditional tests of complete spatial randomness were explored, using the Poisson simulations as empirical null distributions against which alternative distributions were compared. In other words, because the inherent non-uniform nature of outsoles violate one of the principle criteria of CSR (homogeneity), Poisson simulations were created in order to represent the expected distribution of computed CSR test statistics under randomness, rather than the published theoretical underlying distributions (e.g., chi-square, normal, etc.). Ultimately, the goal was to determine whether using traditional approaches to detect departures from randomness were well-powered and suitable for investigating RAC spatial distributions. Power is defined as the probability that a true alternative hypothesis will result in the rejection of the null hypothesis by a given test. Under ideal circumstances, adequate power is generally described as being at least an $80 \%$ true reject rate $($ or $1-4 \alpha)$ ) [34].

Similar to the flat outsole testing, quadrat and distance methods were again implemented in order to determine the power of common techniques for evaluating adherence to complete spatial randomness on both shoe-specific (500 randomly selected simulations from one shoe, possessing the maximum number of RACs (465)) and heatmap-wide data (1,000 randomly selected heatmap simulations, aggregating data from all 1,300 shoes). In other words, for the shoe-specific tests, 500 Poisson, clustered (in bin), and dispersed (out of bin) simulations were randomly selected for the given shoe (thought to be the best-case scenario given the maximum number of features) and each alternative distribution was compared against the Poisson distributions. This same approach was taken for the heatmap-wide evaluations, with 1,000 realizations of each distribution being selected. Given the lower sensitivity of quadrat methods owing to their inherent data binning, a single, widely-used technique referred to as quadrat counting was employed. In addition, three distance methods were conducted at 5 distance orders: Pollard's distance, Pielou's distance, and Clark and Evan's distance. 


\section{Quadrat Counting Approach}

Quadrat counting entails comparing a dataset of empirical spatial events (binned into quadrats) to a random dataset of events that are plotted over the same area, with the same number of quadrats $[21,35]$. In order to conduct this test, a frequency (and cumulative frequency) table was constructed detailing the number of cells with a given count of events for both the empirical and random datasets; likewise, the absolute differences in cumulative proportions $(|\Delta|)$ between the empirical and random data were also computed. The data in the constructed table was then subjected to a Kolmogorov-Smirnov (KS) test to evaluate the presence of statistically significant differences between the observed frequencies and those expected from a random dataset. Deviation was assessed by comparing a test statistic (Eq. 8, or the maximum absolute difference between the observed (empirical) and expected (random) cumulative frequencies), versus a critical value, computed according to Eq. 9, where $m$ is the number of quadrats in each dataset (987 for this study) [35].

$$
D=\max |\Delta|
$$

$$
D(\alpha=0.05)=1.36 \sqrt{\frac{2 m}{m^{2}}}
$$

Comparison of the test statistic to the critical value allowed for a conclusion regarding the null hypothesis (no statistical difference between the empirical and random datasets). More specifically, if the test statistic was greater than the critical value, the null hypothesis was rejected, and the observed deviation cannot be explained by sampling alone.

\section{Pollard's Distance Statistic}

Pollard's distance statistic was computed in the same manner as previously described (Eq. 7), with two exceptions. First, for the shoe-specific assessments, 50 random points were projected (instead of 100) given the smaller sample size of RACs in the dataset. Second, only one set of random points was generated per simulation since this was a preliminary evaluation, thereby excluding Step 4 previously described. This procedure was completed for each Poisson realization and each of the alternative distributions. Subsequently, the 
resulting set of test statistics from the null distributions was used to determine the upper and lower bounds of the expected values (95\% confidence interval) of Pollard's statistic under randomness. Each value of $P_{o}$ (Eq. 7) obtained from the alternative distributions was compared to this confidence interval and any values falling outside of this interval were deemed significant (indicating evidence to reject the null hypothesis of CSR) [20, 28, 36].

\section{Pielou's Distance Statistic}

Similar to Pollard's distance, Pielou's distance statistic $(P)$ likewise evaluates spatial patterns using distances between synthetically generated random points $(i)$ and the $j$ thnearest observed events (RACs in this case) [20, 28, 37]. This test statistic is powerful for detecting departures from CSR at low distance orders (i.e., for RACs that are close together). For this study, Pielou's test statistic was computed using the following procedure, where all variables are defined the same as in Eq. 7 and $\lambda$ represents the population intensity (points per unit area) [20, 28, 37]:

1. Randomly project $n$ synthetic points onto the standard heatmap outsole, where $n=50$ for shoe-specific analysis and $n=100$ for heatmap-wide assessments.

2. In order to adjust for edge effects, an edge correction procedure was incorporated as follows. For each synthetic point $(i)$, find its 5 th nearest neighbor (simulated RAC $\left.\left(x_{i 5}\right)\right)$ as well as the distance from the point to the perimeter of the study region $\left(x_{i p}\right)$. If $x_{i p} \leq x_{i 5}$, then delete this point and re-project a new point, again checking $x_{i 5}$ versus $x_{i p}$

3. Compute Pielous's test statistic $(P)$ for $j=1$, according to Eq. 10 .

$$
P=\pi \lambda \sum_{i=1}^{n} \frac{x_{i j}^{2}}{j n}
$$

4. Repeat steps 1-3 for each distance order $(j=2,3,4,5)$.

Again, each resulting $P$ statistic obtained from the Poisson random simulations were aggregated and a 95\% confidence interval for this test statistic was determined for the null 
distribution. Lastly, each Pielou's distance statistic obtained for both alternative distributions was assessed for deviation from complete spatial randomness by comparing it to the simulated $95 \%$ significance range obtained using the Poisson distributions.

\section{Clark and Evan's Distance Statistic}

Unlike the two previous methods, the Clark and Evans' statistic $(C)$ evaluates the distance between a randomly selected $R A C(i)$ and its $j$ th-nearest RAC neighbors. The general framework for using the Clark and Evans' statistic to test for departure from CSR is as follows, with all variables defined as in the previous detailed distance approaches [20, 28, 30]:

1. Randomly select $n$ RACs from the simulated point process, where $n=50$ for shoespecific analysis and $n=100$ for heatmap-wide assessments.

2. For each randomly selected RAC $(i)$, find its 5th nearest neighbor (simulated RAC $\left.\left(x_{i 5}\right)\right)$ as well as the distance from the point to the perimeter of the study region $\left(x_{i p}\right)$. If $x_{i p} \leq x_{i 5}$, then delete this point and re-project a new point, again checking $x_{i 5}$ versus $x_{i p}$. This step serves as the edge correction for this approach.

3. Compute Clark and Evans' test statistic $(C)$ for $j=1$, according to Eq. 11.

$$
C=2^{2 j} \sqrt{\lambda} \sum_{i=1}^{n} \frac{x_{i j}}{j n\left(\begin{array}{c}
2 j \\
j
\end{array}\right)}
$$

4. Repeat steps 1-3 for each distance order $(j=2,3,4,5)$.

Similarly, a 95\% confidence interval was obtained using the set of calculated Clark and Evan's distance statistics from the Poisson random simulations and each resulting $C$ value from the alternative distributions was compared to this range in order to evaluate adherence to CSR.

\section{Testing for CSR Adjusted for Spatial Inhomogeneity}

Although the previously described techniques are extensively used to determine whether spatial data adheres to CSR, complete spatial randomness is rarely ever an appropriate description in practicality [23, 38-41]. For example, when studying the distribution of tree species, they are much less likely to fall in a body of water or on a road than in soil. The 
same is true of the RACs under investigation here; they cannot be observed on the recessed areas of outsoles in two-dimensional impressions. Very often this stationarity requirement of CSR, wherein points are equally likely to fall anywhere within the study region causing a uniform intensity across the entire region, is violated. Therefore, there is a need to adjust for this underlying inhomogeneity within the sample space in order to properly ascertain the relationships between points at various distances as a function of the variation in expected point intensity [38]. In fact, the development of robust approaches to evaluate adherence to randomness in data collected over non-uniform study regions is currently receiving great research interest in spatial statistics, with Ripley's K being the most commonly cited method that accounts for non-stationarity in data [38-41].

The inhomogeneous Ripley's $\mathrm{K}\left(\hat{K}_{i n h}\right)$ is a second order spatial statistic that not only evaluates the mean of a parameter across space (e.g., intensity, distance, etc. as in the previously outlined methods) but also the variance of the estimate in the sampling area $[20,23,39-41]$. In order to evaluate the performance of this approach for footwear features, a small sample of 4 database outsoles was selected. These shoes contained a relatively large number of RACs $(83,90,101,114)$ and various class characteristics (circles and small bars, large lugs, small lugs, and stars and bars), thus allowing for an assessment of variations in contact area that are exhibited by different types of outsoles within the WVU database. For each shoe, its empirically observed RACs, as well as 500 randomly selected Poisson, clustered (in bin) and dispersed (out of bin) simulations, were utilized for analysis.

Inhomogeneous Ripley's K was computed for each shoe, using each of the three simulated distributions, according to Eq. $12[40,41]$, where $A$ represents the area of the region of interest (number of "on" contact pixels), $w_{i j}$ represents an edge correction factor, $t$ is the radius of interest, $\lambda_{i}$ and $\lambda_{j}$ are the estimated point intensities at each RAC of interest $\left(x_{i}\right.$ and $x_{j}$, respectively), and $x_{i j}$ represents the absolute Euclidean distance between them. For the purposes of this study, point intensities were estimated using an edge-corrected Gaussian kernel density estimator (KDE) with bandwidth equal to 200px (8.3mm) (the average radius of lugs), where the KDE edge correction factor was defined as the inverse of the area of the kernel located on top of contact pixels. In addition, note that the edge correction factor for computation of Ripley's K (Eq. 12) was set to 1 because edge correction was previously 
applied in the estimation of intensity. Furthermore, given that this test statistic is typically evaluated across a variety of radii, $\hat{K}_{i n h}$ was computed at $t=0,25,50, \ldots, 1,000$.

$$
\begin{aligned}
\hat{K}_{i n h} & =\frac{1}{A} \sum_{i=1}^{N} \sum_{j \neq i}^{N} \frac{w_{i j} I\left(x_{i j} \leq t\right)}{\lambda\left(x_{i}\right) \lambda\left(x_{j}\right)} \\
I\left(x_{i} j \leq t\right) & = \begin{cases}1 & \text { if } x_{i j} \leq t \\
0 & \text { otherwise }\end{cases}
\end{aligned}
$$

Traditionally, interpretation of this metric is conducted by generating a confidence envelope of $\mathrm{K}$ values ( $95 \%$ confidence interval at each value of $t$ ) and plotting this along with the similarly obtained resulting empirical data (in this case, tests based on the distribution of observed RACs on each shoe). If the empirical data falls within the confidence envelope at any given radius, it exhibits CSR at that scale. Conversely, if the observations lie above the upper limit of the confidence band, the data exhibit clustering at the given radius (and vice versa for patterns of dispersion falling below the lower limit) [23, 24, 39-41]. While this approach was conducted for this research, the confidence bands for the clustered and dispersed simulation results were likewise plotted along with the null distribution and empirical data.

\section{Global Evaluation of Spatial Autocorrelation}

Rather than directly assessing the presence/absence of randomness and instead moving towards characterization of a spatial distribution, spatial autocorrelation evaluates the level of independence of spatial samples by assessing not only the location of the events (by binning into regions/quadrats) but also by a measured attribute at that location (number of RACs overall, presence of RACs of a given shape, errors/residuals, etc.) [35, 42]. More specifically, this technique considers the degree to which points are similar to other points in a given area. A positive spatial autocorrelation indicates that similar features cluster together in space, while a negative correlation indicates that dissimilar features cluster together in space $[35,43]$. For this study, evaluation of global autocorrelation was conducted by applying a widely-used statistic referred to as Moran's I [23, 24, 35, 43-45]. In order to compute this metric, the following procedure was used, where $x$ represents the feature of interest at a specific location and $Q$ represents the number of quadrats ( $Q=987$ heatmap bins): 
1. Compute the Euclidean distance between the centroids of each quadrat in the heatmap.

2. Construct a row-normalized weight matrix $(W)$ using the inverse of the Euclidean distances for all pairs of quadrats, wherein the sum across each row is one. If desired, a distance cut-off can be specified beyond which all weights are assigned a value of zero. For the analysis of global autocorrelation for this study, no cut-off was selected thus allowing all bins to serve as neighbors.

3. Compute Moran's I, according to Eq. 13 [35, 43].

$$
\begin{aligned}
S_{0} & =\sum_{i} \sum_{j} w_{i j} \\
I & =\frac{Q}{S_{0}} * \frac{\sum_{i} \sum_{j} w_{i j}\left(x_{i}-\bar{x}\right)\left(x_{j}-\bar{x}\right)}{\sum_{i}\left(x_{i}-\bar{x}\right)^{2}}
\end{aligned}
$$

4. Compute the expected value $(E(I))$ and variance $(\operatorname{Var}(I))$, according to Eq. 14 [35, 43$]$.

$$
\begin{aligned}
S_{1} & =\frac{1}{2} \sum_{i} \sum_{j}\left(w_{i j}+w_{j i}\right)^{2} \\
S_{2} & =\sum_{i}\left(w_{i 0}+w_{0 i}\right)^{2} \\
w_{i 0} & =\sum_{j} W_{i j} \\
w_{0 i} & =\sum_{j} W_{j i} \\
k & =\frac{\sum_{i=1}^{n}\left(x_{i}-\bar{x}\right)^{4}}{\left(\sum_{i=1}^{n}\left(x_{i}-\bar{x}\right)^{2}\right)^{2}} \\
\operatorname{Var}(I) & =\frac{Q\left[\left(Q^{2}-3 Q+3\right) S_{1}-Q S_{2}+3 S_{0}^{2}\right]-k\left[Q(Q-1) S_{1}-2 Q S_{2}+6 S_{0}^{2}\right]}{(Q-1)(Q-2)(Q-3) S_{0}^{2}} \frac{1}{(Q-1)^{2}} \\
E(I) & =\frac{-1}{Q-1}
\end{aligned}
$$

5. Compute the standardized Z score, according to Eq. 15, and compare to the critical value.

$$
Z=\frac{I-E(I)}{69}
$$


Although Moran's statistic follows an approximately normal distribution, this approach was selected because it has been shown to be very robust against departures from normality $[23,24]$. Additionally, values significantly lower than expected indicate negative correlation (dissimilar RAC counts in close proximity) and conversely values significantly higher than expected suggest positive correlation (similar RAC counts in close proximity).

More specifically, spatial autocorrelation was assessed on a set of "observed residuals" ( $x$ 's in Eq. 13), wherein the Poisson simulation heatmaps served as the "predicted" outcomes (or modeled/expected) against which empirical data were compared. However, since this method considers all locations (bins) equal, it was necessary to first normalize RAC counts $\left(n_{m}\right)$ in order to account for both bin partiality $\left(B_{p}\right.$, or the percentage of the bin that falls within the heatmap frame) and contact partiality $\left(C_{p}\right.$, or the mean percentage of the bin that was covered by contact area across all 1,300 shoes). These modified frequencies were computed according to Eq. 16 and served to normalize counts in incomplete cells in order to reflect what they would be given full contact (covering all 14,161 pixels for a $5 \mathrm{~mm} \times 5 \mathrm{~mm}$ quadrat contained entirely within the heatmap frame).

$$
\begin{aligned}
B_{p} & =\frac{\# \text { px on frame }}{14,161} \\
C_{p} & =\frac{\sum_{1}^{1,300} \# \text { px in contact }}{14,161 \times 1,300} \\
n_{m} & =\frac{\# \text { RACs in bin }}{\frac{C_{p}}{B_{p}}}
\end{aligned}
$$

Furthermore, this assessment was conducted in three phases: (i.) Poisson versus Poisson simulations (a null distribution check for Type I error), (ii.) alternatives versus Poisson simulations (an alternative distribution check for power), and lastly (iii.) empirical data versus Poisson simulations (to characterize the WVU database RACs), according to Table 1. The residuals were obtained by subtracting the predicted values $\left(n_{m e}\right)$ from the observed values $\left(n_{m o}\right)$, such that $x=n_{m o}-n_{m e}$ for each bin, and these values were used in the calculation of global Moran's I. 
Table 1: Total number of comparisons and distributions of interest for each phase of the global autocorrelation analysis using Moran's I. Note that for the assessment of Type I error, 10 Poisson distributions were randomly selected and all were pairwise compared to yield 45 total comparisons $((10 \times 9) / 2)$. For the power analysis and evaluation of RAC residuals, each randomly selected observed distribution was compared against all randomly selected predicted distributions to yield 100 total comparisons $(10 \times 10)$.

\begin{tabular}{|c|c|c|c|}
\hline Assessment & $\begin{array}{c}\text { Observed } \\
\text { Distribution }\end{array}$ & $\begin{array}{c}\text { Predicted } \\
\text { Distribution/ }\end{array}$ & $\begin{array}{c}\text { Total Number } \\
\text { of Comparisons }\end{array}$ \\
\hline Type I Error & Poisson: 10 & Poisson: same 10 & 45 \\
\hline \multirow{2}{*}{ Power } & Clustered: 10 & Poisson: 10 & 100 \\
& Dispersed: 10 & Poisson: 10 & 100 \\
\hline RAC Count Residuals & Empirical: 1 & Poisson: 100 & 100 \\
\hline
\end{tabular}

\section{Local Indicators of Spatial Autocorrelation}

Although global assessment of spatial correlation is useful for evaluating the overall pattern, local indicators of spatial association (LISAs) decompose the global metric in pursuit of a more descriptive characterization of spatial data, thereby allowing for identification of localized regions of significant correlation [23, 46]. Rather than obtaining a single statistic, this analysis yields one estimate per cell of interest (987 in this study) to describe localized spatial relationships. Local Moran's I was therefore utilized in an attempt to characterize the distribution of RACs on shoe outsoles. This technique was again employed to evaluate the "observed residuals" between the empirical data and all 10,000 Poisson random simulations in order to determine if specific regions of the outsole are not well predicted by the random realizations. To do so, the same approach was employed as described for global Moran's I, but substituting Eq. 17 for the computation of the local test statistic $\left(I_{i}\right)$ and its associated expected value and variance. In addition, this assessment was conducted using six different neighborhood distance cut-off values (400px or $16.7 \mathrm{~mm}, 800 \mathrm{px}$ or $33.3 \mathrm{~mm}, 1,000 \mathrm{px}$ or $41.7 \mathrm{~mm}, 1,500 \mathrm{px}$ or $62.5 \mathrm{~mm}, 1,750 \mathrm{px}$ or $72.9 \mathrm{~mm}, 2,500 \mathrm{px}$ or $104 \mathrm{~mm}$ ) in the row-normalized weight matrix in an attempt to glean information about correlation structures at various radii and whether observations are stable at different scales. 


$$
\begin{aligned}
S_{i}^{2} & =\frac{\sum_{j=1}^{Q}\left(x_{j}-\bar{x}\right)^{2}}{Q-1} \\
b & =\frac{Q \sum_{j=1}^{Q}\left(x_{i}-\bar{x}\right)^{4}}{\left(\sum_{i=1}^{Q}\left(x_{i}-\bar{x}\right)^{2}\right)^{2}} \\
I_{i} & =\frac{x_{i}-\bar{x}}{S_{i}^{2}} \sum_{j=1}^{Q} w_{i j}\left(x_{j}-\bar{x}\right) \\
E\left(I_{i}\right) & =-\frac{\sum_{j=1}^{Q} w_{i j}}{Q-1} \\
\operatorname{Var}\left(I_{i}\right) & =\frac{(Q-b) \sum_{j=1}^{Q} w_{i j}^{2}}{Q-1}-\frac{(2 b-Q) \sum_{k=1}^{Q} \sum_{h=1}^{Q} w_{i k} w_{i h}}{(Q-1)(Q-2)}-\left[E\left(I_{i}\right)\right]^{2}
\end{aligned}
$$

\section{Results and Discussion}

\section{Database and Pre-Processing}

Figure 1 details the frequency distribution of the number of RACs identified on all outsoles in the collection with the mean number of features per shoe equal to $56 \pm 64$ with an empirical minimum of 1 and maximum of 465. Given the large skew and spread in the data, the median of 32 (with associated IQR of 14 to 70) is clearly a better estimator of central tendency for this dataset.

After identification of randomly acquired characteristics on each outsole, data for all 1,300 shoes was aggregated into a single frequency heatmap possessing 987 potential bin locations into which these features were discretized. Outlined in Table 2 are the summary statistics for the number of features per heatmap cell overall and broken out by shape class. Disregarding shape, there is an average of $73 \pm 36$ RACs per bin.

Furthermore, RAC and contact area frequencies per heatmap location were combined and are illustrated in Figure 2 where the color of each dot represents the number of features and the size of each dot represents the number of shoes with contact. Upon more detailed examination, it becomes apparent that cell frequencies are likely dependent on contact area which fits with intuition. For example, in the arch area of the standard outsole, there are fewer shoes that possess tread elements in this region $(\sim 25-45 \%)$ and likewise there are very few RACs; conversely, about 80-90\% of outsoles had contact pixels in the ball of the toe and 


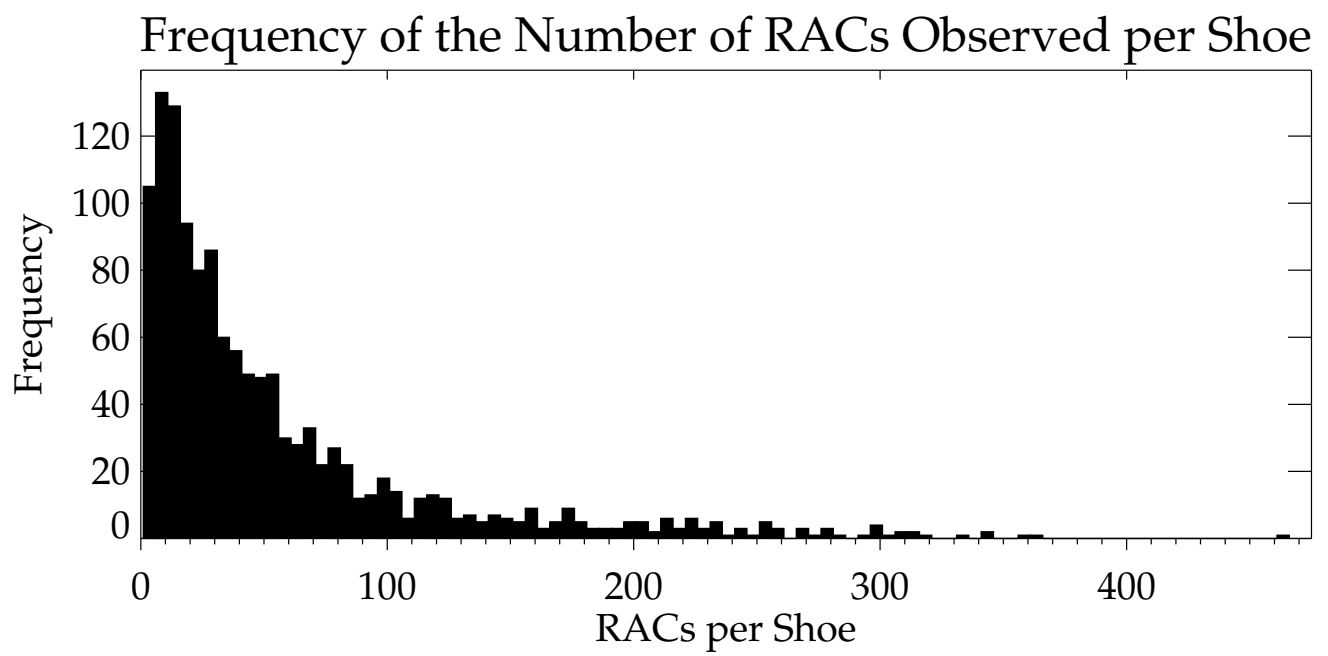

Figure 1: Frequency of the number of RACs observed on all 1,300 shoes.

Table 2: Summary statistics for RAC frequency as a function of heatmap bins. In total, there were 72,306 RACs identified on 1,300 shoes and ultimately categorized as either variable (28,236 RACs), linear (21.363 RACs), or compact (22,707 RACs).

\begin{tabular}{|c|c|c|c|c|}
\hline Metric & Variable & Linear & Compact & Any \\
\hline Mean & 29 & 22 & 23 & 73 \\
\hline SD & 15 & 12 & 12 & 36 \\
\hline Median & 29 & 21 & 24 & 76 \\
\hline IQR & $(19,38)$ & $(13,30)$ & $(14,32)$ & $(48,97)$ \\
\hline Max & 73 & 71 & 57 & 189 \\
\hline
\end{tabular}

this region also has a higher density of accidentals. Interestingly, the accumulated contact areas displayed in Figure 2 exhibit a similar structure to those observed by Yekutieli, et. al., (2013) [18], wherein there is little contact in the interior heel and arch area. Given these reproducible results on two different databases, it seems that this may be a stable model for general contact area of athletic outsoles. Moreover, the results of this preliminary analysis reveal that it is imperative to account for contact versus recessed areas when evaluating the distribution of characteristics of use for forensic footwear impressions. 


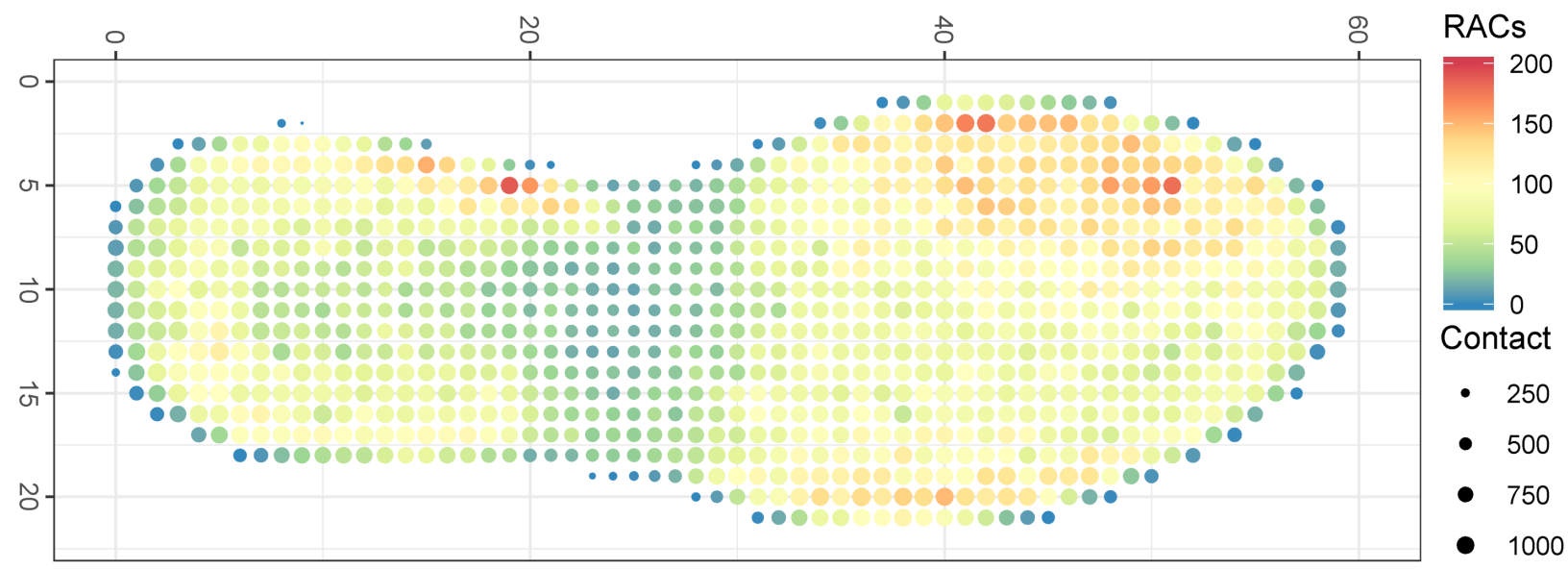

Figure 2: Number of RACs (color) and number of outsoles with contact (point size) for each cell in the WVU database heatmap. Note that for this illustration contact in a given bin was considered a binary decision wherein a shoe's outsole was considered "on" if its binary contact map covered at least $25 \%$ of a bin's area (3540px or $\sim 150 \mathrm{~mm}^{2}$ for a full bin) and/or a RAC was observed in that location.

\section{Point Process Simulation}

Using the binary contact maps as a window in which simulated points can fall, a series of Poisson, clustered, and dispersed point process realizations were created for each shoe. In total, $3,748,251$ contact-modified accidental feature simulations have been generated, as detailed in Table 3. These simulations (or random sub-samples of them) were used for all data analysis moving forward, and serve as the basis against which to compare the empirical data.

\section{Poisson Simulation Proof of Concept: Flat Outsole Testing}

Apart from the contact-modified simulations, 2,000 flat outsole Poisson realizations were created using the completely filled frame of the standard outsole as the window within which 72,306 points were generated; Figure 3 displays one such simulation. Not surprisingly, when comparing this figure to Figure 2 there are apparent differences between the two, including the lack of underlying structure in the flat outsole simulations. Additionally, RAC counts seem to be relatively uniformly distributed, with the exception of partial bins along the edge of the frame which possess fewer features, as expected.

Each of the 2,000 Poisson realizations was subjected to four traditional tests of complete 
Table 3: Number of contact-area modified, shoe-specific Poisson and alternative simulations created for this study.

\begin{tabular}{|c|c|c|c|c|}
\hline Simulation & $\begin{array}{c}\text { Desired \# } \\
\text { per Shoe } \\
\text { (minimum) }\end{array}$ & Pattern Factor(s) & \# Created & $\begin{array}{c}\text { Mean \# } \\
\text { per Shoe }\end{array}$ \\
\hline Poisson & 1,000 & Intensity $(\lambda)$ & $1,764,792$ & 1357 \\
\hline $\begin{array}{c}\text { Clustered } \\
\text { in Bin }\end{array}$ & 500 & Intensity $(\lambda)$ & 650,000 & 500 \\
\hline $\begin{array}{c}\text { Clustered } \\
\text { on Lug }\end{array}$ & 500 & $\begin{array}{c}\text { Number points/cluster }\left(n_{c}\right): 2-6 \\
\text { Clustering radius }\left(r_{c}\right): 85 \mathrm{px}(3.5 \mathrm{~mm})\end{array}$ & 501 \\
\hline $\begin{array}{c}\text { Dispersed } \\
\text { out of bin }\end{array}$ & 500 & $\begin{array}{c}\text { Intensity }(\lambda) \\
\text { (Incremented by } 5 \mathrm{px}(\sim 0.2 \mathrm{~mm}))\end{array}$ & 651,712 & 524 \\
\hline $\begin{array}{c}\text { Dispersed } \\
\text { off Lug }\end{array}$ & 500 & $\begin{array}{c}\text { Inhibition radius }\left(r_{i}\right): 85 \mathrm{px}(3.5 \mathrm{~mm}) \\
\text { allowable dispersion } 400-75 \mathrm{px}(16.7-3.1 \mathrm{~mm})\end{array}$ & In progress & In progress \\
\hline
\end{tabular}

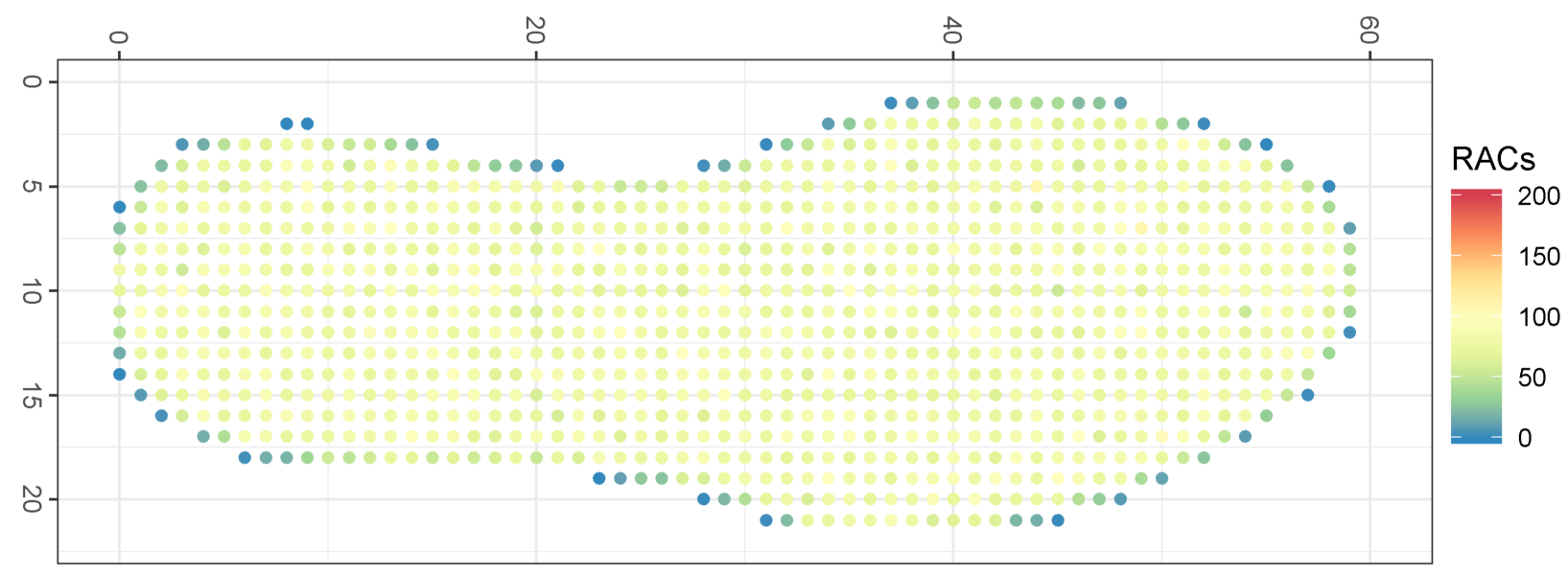

Figure 3: Number of RACs (color) for each cell location for a randomly selected flat outsole Poisson simulation.

spatial randomness (stemming from two classes of techniques) in order to ascertain whether the procedure for creating the null distribution simulations actually produced point processes that conformed to CSR. Under the null hypothesis, $p$-values are uniformly distributed [47]; therefore, it was expected that approximately $5 \%$ of tests conducted on the flat outsole data would reject randomness at $\alpha=0.05$. Table 4 reports the results based on four techniques 
(chi-square GOF, paired quadrat, ANN, and Pollard's distance), yielding rejection rates ranging from 4.0-6.2\%. Most notably, the mean rejection rate for all tests conducted was $4.9 \%$, which aligns with the expected proportion under the null hypothesis. Therefore, these results offer evidence that the methodology for generating the Poisson simulations are, in fact, spatially random and can reliably be used to serve as null distributions moving forward.

Table 4: Results of flat outsole simulation testing based on 2,000 heatmap realizations with four commonly used tests of spatial randomness (representing both quadrat and distance methods). Note for the paired quadrat approach, cells that were between 2-5 bins apart were randomly paired for analysis. In addition, for the Pollard statistic, five distance orders were evaluated (i.e., the distances between each selected point and its five nearest neighbors).

\begin{tabular}{|c|c|c|c|c|c|}
\hline \multicolumn{2}{|r|}{ Method } & $\begin{array}{l}\text { Approximate } \\
\text { Distribution }\end{array}$ & $\begin{array}{c}\text { \# Samples } \\
\text { per Simulation }\end{array}$ & \# Tests & $\begin{array}{c}\text { Reject } \\
\text { CSR }\end{array}$ \\
\hline \multirow{2}{*}{ 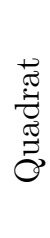 } & $\begin{array}{c}\text { Chi-square } \\
\text { GOF }\end{array}$ & $\begin{array}{c}\text { Chi-square } \\
(\mathrm{df}=\# \text { bins-1) }\end{array}$ & $\mathrm{N} / \mathrm{A}$ & 2,000 & $4.7 \%$ \\
\hline & $\begin{array}{c}\text { Paired } \\
\text { Quadrat }\end{array}$ & $\begin{array}{c}\text { Chi-square } \\
(\mathrm{df}=\# \text { dists-1) }\end{array}$ & $\begin{array}{c}1,000 \\
(4 \text { dists, } 50 \text { points })\end{array}$ & $2,000,000$ & $4.7 \%$ \\
\hline \multirow[b]{2}{*}{ 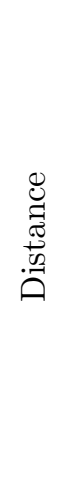 } & $\begin{array}{c}\text { Average } \\
\text { NN }\end{array}$ & $\begin{array}{c}\text { Normal } \\
(\mu=1 /(2 \sqrt{\lambda})) \\
(S E=0.26136 / \sqrt{N \lambda})\end{array}$ & $\mathrm{N} / \mathrm{A}$ & 2,000 & $6.2 \%$ \\
\hline & Pollard & $\begin{array}{c}\text { Chi-square } \\
(\mathrm{df}=\# \text { points- } 1)\end{array}$ & $\begin{array}{c}1,000 \\
\text { (100 random points) }\end{array}$ & $2,000,000$ & $\begin{array}{l}\text { Dist 1: } 4.0 \% \\
\text { Dist 2: } 4.8 \% \\
\text { Dist 3: } 5.0 \% \\
\text { Dist 4: } 5.0 \% \\
\text { Dist 5: } 5.0 \%\end{array}$ \\
\hline
\end{tabular}

\section{Power Testing of Traditional CSR Tests on Contact-Area Modified Simulations}

As the first step in characterizing the distribution of RACs on non-uniform outsoles, traditional and widely-used spatial statistical approaches to testing for complete spatial randomness were employed. Prior to testing empirical data obtained from the collection of 1,300 shoes, the power of these tests for contact-modified simulations (which appropriately represent the regions of interest for this study of outsoles) was evaluated on shoe-specific and heatmap-wide scales. Namely, the shoe-specific level was comprised of 500 simulations 
(for each pattern type) generated for a single shoe containing 465 RACs (the most features contained on any shoe in the database). Alternatively, the heatmap-wide level was comprised of 1,000 simulated heatmaps (for each pattern type) representing all 72,306 synthetic RACs, modified by the contact areas of 1,300 outsoles.

First, within-distribution testing was completed on the 500 shoe-specific simulations, for all three distributions (Poisson, clustered, and dispersed) using a hold-one-out analysis whereby the remaining realizations were used to inform an empirical $95 \%$ confidence interval against which to compare the singular result (Table 5). Theoretically, each held-out realization should fall within the significance range, thereby indicating that the specific data originates from the distribution as the "null" data (the 499 remaining point processes), with an error rate of approximately 5\%. With the exception of the quadrat counting technique, which is known to have low sensitivity for small departures owing to data binning, all other tests exhibited $5.2-5.6 \%$ rejection which closely adheres to the expected error rate suggesting that these approaches have suitable specificity, at least when comparing like-simulations.

Following within-distribution assessment, between-distribution analysis was undertaken for both the shoe-specific and heatmap-wide scales using the same four tests of spatial randomness. For each of these assessments, the Poisson random simulations served as the null distribution from which an empirical confidence interval was derived using the computed test statistics; each alternative distribution result was then compared to this range to determine adherence to CSR. Theoretically, each alternative distribution should fall outside the significance range determined using the Poisson simulations as the null, with an error rate of no more than $20 \%$ thus yielding a power of $80 \%$ or higher. Table 6 details the computed power (true rejection rate) for shoe-specific and heatmap-wide tests of spatial randomness for clustered and dispersed alternative distributions. Overall, the results of power testing for traditional tests of CSR exhibit low performance for assessment of RAC distributions on contact-modified imagery, specifically for dispersed simulations and heatmap-wide scales.

When considering the dispersed simulations, a maximum true rejection rate of approximately $15 \%$ was observed, with a mean power of $4.4 \% \pm 4.0 \%$ (Table 6 ). Although all three distance-based test statistics are known to be slightly more sensitive to clustered deviations from randomness [28], the results are lower than expected for the dispersed alternative. 
Table 5: Frequency (and associated percentage) of falsely rejected distributions for hold-one-out assessment of within-distribution testing on shoe-specific contact-modified simulations using traditional tests for CSR. The distance-based tests were conducted using five distance orders $(j=1-5)$, where the distances between a randomly selected point and its $j$ th nearest neighbor were evaluated. When appropriate, the distance order is also noted in the table.

\begin{tabular}{|c|c|c|c|}
\hline Distribution & Poisson & $\begin{array}{l}\text { Clustered } \\
\text { (in bin) }\end{array}$ & $\begin{array}{l}\text { Dispersed } \\
\text { (out of bin) }\end{array}$ \\
\hline Scale & Shoe & Shoe & Shoe \\
\hline $\begin{array}{l}\text { Quadrat } \\
\text { Counting }\end{array}$ & $0(0.0)$ & $0(0.0)$ & $0(0.0)$ \\
\hline Pollard & $\begin{array}{lll}j=1: & 27 & (5.4) \\
j=2: & 27 & (5.4) \\
j=3: & 28 & (5.6) \\
j=4: & 26 & (5.2) \\
j=5: & 28 & (5.6)\end{array}$ & 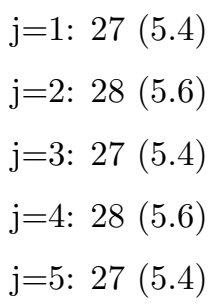 & $\begin{array}{lll}\mathrm{j}=1: & 27 & (5.4) \\
\mathrm{j}=2: & 26 & (5.2) \\
\mathrm{j}=3: & 28 & (5.6) \\
\mathrm{j}=4: & 27 & (5.4) \\
\mathrm{j}=5: & 28 & (5.6)\end{array}$ \\
\hline Pielou & $\begin{array}{lll}j=1: & 27 & (5.4) \\
j=2: & 27 & (5.4) \\
j=3: & 26 & (5.2) \\
j=4: & 27 & (5.4) \\
j=5: & 27 & (5.4)\end{array}$ & $\begin{array}{lll}j=1: & 26 & (5.2) \\
j=2: & 27 & (5.4) \\
j=3: & 26 & (5.2) \\
j=4: & 26 & (5.2) \\
j=5: & 27 & (5.4)\end{array}$ & $\begin{array}{lll}\mathrm{j}=1: & 26 & (5.2) \\
\mathrm{j}=2: & 28 & (5.6) \\
\mathrm{j}=3: & 26 & (5.2) \\
\mathrm{j}=4: & 26 & (5.2) \\
\mathrm{j}=5: & 27 & (5.4)\end{array}$ \\
\hline Clark \& Evans & 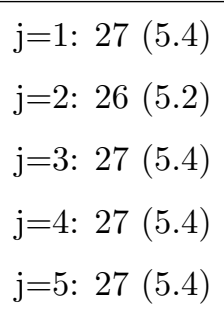 & 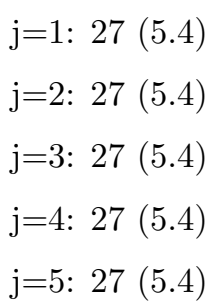 & 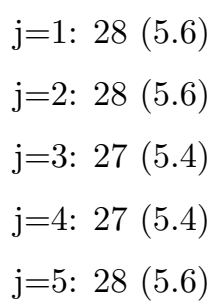 \\
\hline
\end{tabular}

Therefore, this observation suggests that the dispersion factor for out of bin (85px or 3.5mm) is likely not sufficiently large for many shoes in the database, resulting in the intended alternative distribution adhering to an approximately random pattern. As such, it was at this point that additional dispersed simulations were created in an attempt to better represent dispersion outside of lugs using the maximum allowable radius, as previously summarized in Table 3 .

Furthermore, a large decrease in power is observed between the shoe-specific and heatmaplevel scales, specifically in the case of the clustered distributions. On average, the shoe- 
Table 6: Frequency (and associated percentage) of true rejected distributions for between-distribution testing on shoe-specific (500 each) and heatmap-wide (1,000 each) contact-modified simulations using traditional tests for CSR, with the Poisson random simulations serving as the empirical null distribution. The distancebased tests were conducted using five distance orders $(j=1-5)$, where the distances between a randomly selected point and its $j$ th nearest neighbor were evaluated. When appropriate, the distance order is also noted in the table.

\begin{tabular}{|c|c|c|c|c|}
\hline Distribution & $\begin{array}{c}\text { Clustered } \\
\text { (in bin) }\end{array}$ & $\begin{array}{c}\text { Dispersed } \\
\text { (out of bin) }\end{array}$ & $\begin{array}{c}\text { Clustered } \\
\text { (in bin) }\end{array}$ & $\begin{array}{c}\text { Dispersed } \\
\text { (in bin) }\end{array}$ \\
\hline Scale & Shoe & Shoe & Heatmap & Heatmap \\
\hline $\begin{array}{c}\text { Quadrat } \\
\text { Counting }\end{array}$ & $282(56)$ & $1(0.2)$ & $10(2.0)$ & $0(0.0)$ \\
\hline \multirow{5}{*}{ Pollard } & $\mathrm{j}=1: 78(16)$ & $\mathrm{j}=1: 21(4.2)$ & $\mathrm{j}=1: 38(3.8)$ & $\mathrm{j}=1: 32(3.2)$ \\
& $\mathrm{j}=2: 219(44)$ & $\mathrm{j}=2: 57(11)$ & $\mathrm{j}=2: 77(7.7)$ & $\mathrm{j}=2: 17(1.7)$ \\
& $\mathrm{j}=3: 263(53)$ & $\mathrm{j}=3: 72(14)$ & $\mathrm{j}=3: 85(8.5)$ & $\mathrm{j}=3: 21(2.1)$ \\
& $\mathrm{j}=4: 258(52)$ & $\mathrm{j}=4: 69(14)$ & $\mathrm{j}=4: 83(8.3)$ & $\mathrm{j}=4: 29(2.9)$ \\
& $\mathrm{j}=5: 266(53)$ & $\mathrm{j}=5: 76(15)$ & $\mathrm{j}=5: 104(10)$ & $\mathrm{j}=5: 21(2.1)$ \\
\hline \multirow{5}{*}{ Pielou } & $\mathrm{j}=1: 327(65)$ & $\mathrm{j}=1: 37(7.4)$ & $\mathrm{j}=1: 36(3.6)$ & $\mathrm{j}=1: 14(1.4)$ \\
& $\mathrm{j}=2: 239(48)$ & $\mathrm{j}=2: 35(7.0)$ & $\mathrm{j}=2: 56(5.6)$ & $\mathrm{j}=2: 18(1.8)$ \\
& $\mathrm{j}=3: 164(33)$ & $\mathrm{j}=3: 18(3.6)$ & $\mathrm{j}=3: 66(6.6)$ & $\mathrm{j}=3: 36(3.6)$ \\
& $\mathrm{j}=4: 113(23)$ & $\mathrm{j}=4: 28(5.6)$ & $\mathrm{j}=4: 67(6.7)$ & $\mathrm{j}=4: 14(1.4)$ \\
& $\mathrm{j}=5: 115(23)$ & $\mathrm{j}=5: 20(4.0)$ & $\mathrm{j}=5: 66(6.6)$ & $\mathrm{j}=5: 21(2.1)$ \\
\hline \multirow{5}{*}{ Clark \& Evans } & $\mathrm{j}=1: 312(63)$ & $\mathrm{j}=1: 39(7.8)$ & $\mathrm{j}=1: 42(4.2)$ & $\mathrm{j}=1: 27(2.7)$ \\
& $\mathrm{j}=2: 193(39)$ & $\mathrm{j}=2: 21(4.2)$ & $\mathrm{j}=2: 49(4.9)$ & $\mathrm{j}=2: 26(2.6)$ \\
& $\mathrm{j}=4: 118(24)$ & $\mathrm{j}=3: 6(1.2)$ & $\mathrm{j}=3: 54(5.4)$ & $\mathrm{j}=3: 38(3.8)$ \\
& $\mathrm{j}=5: 55(11)$ & $\mathrm{j}=5: 12(2.4)$ & $\mathrm{j}=5: 44(4.4)$ & $\mathrm{j}=5: 24(2.4)$ \\
\hline
\end{tabular}

specific clustered simulations showed evidence of departures from randomness in $38.6 \% \pm$ $18.1 \%$ of all comparisons, whereas the heatmap-wide level rejected just $5.8 \% \pm 2.1 \%$ (Table $6)$. One possible explanation for this result is that aggregation of data from shoe-specific simulations into random heatmap realizations causes suppression of the underlying patterns, thus making deviations from randomness increasingly difficult to detect. This phenomena is illustrated in Figure 4 wherein the RACs observed in a randomly selected realization for each simulation type (Poisson, clustered, and dispersed) are plotted as heatmaps. Although 

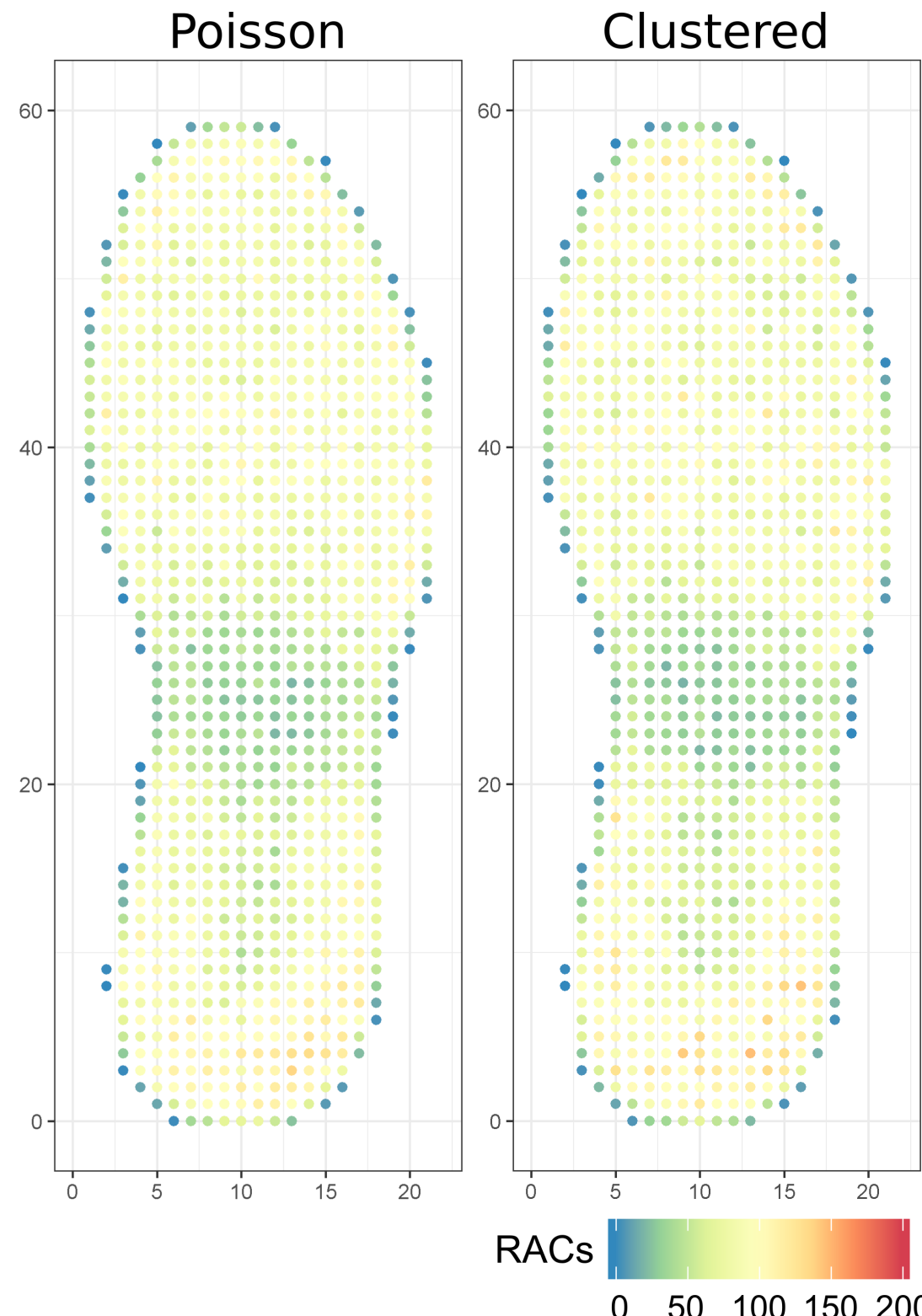

Clustered

50100150200 dispersed, respectively), visual differences are not readily apparent.
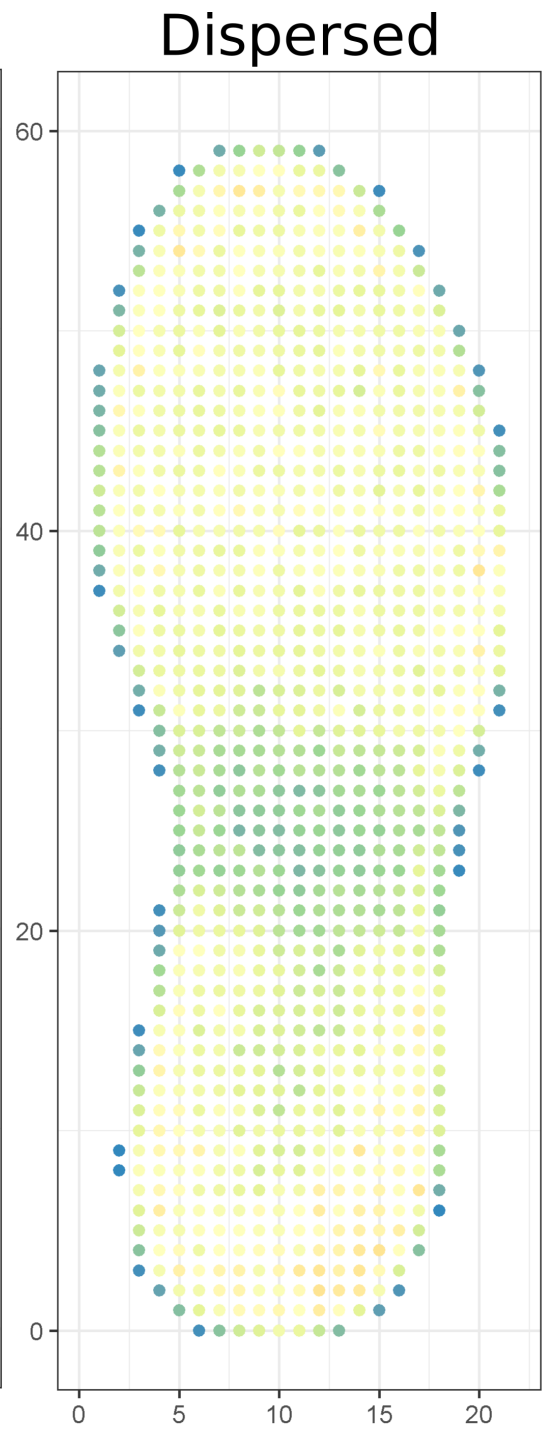

Figure 4: Number of RACs (color) per bin in one (of 10,000) randomly-selected heatmap realization for Poisson, clustered (in bin), and dispersed (out of bin) simulations.

differences do exist between all three distributions (e.g., the maximum number of RACs per bin are highest (144), as expected, for clustered, followed by 137 and 129 for Poisson and 
exhibit the highest power for nearest-neighbor analysis (63-65\% for clustered and 7.4-7.8\% for dispersed alternatives), whereas Pollard's test performs best at higher distance orders (increasing from $16 \%$ to $53 \%$ for clustered and $4.2 \%$ to $15 \%$ for dispersed). Unfortunately, given the vastly different study region size $(12,748,081 \mathrm{px}$ versus $62,500 \mathrm{px})$, the resulting population intensity (0.006 point/px versus 1.000 point/px), and the underlying non-stationarity of the contact-area windows comprising the database, direct comparison of power values between this study and Liu (2001) [28] is not possible.

As previously discussed, traditional tests of CSR exhibit relatively low power for evaluations of RACs contained within contact-modified outsole simulations on multiple scales, with none of the scenarios reaching the $80 \%$ ideal threshold [34]. There are two explanations that likely contribute to this under-performance. First, although point processes were generated for each specific pattern (or lack thereof) onto binary contact windows, thereby describing the sample space within which RACs could fall for each shoe, the study-wide area of interest was the standard heatmap outsole. Within this study area, there still remains inhomogeneity (as exhibited in Figure 2) and therefore one of the principle criteria for complete spatial randomness is inherently violated. Second, each test statistic was developed to possess adequate power following a theoretical distribution for uniform study regions under the null hypothesis. By creating an empirically derived null distribution accounting for tread elements, the power may be compromised because it changes the underlying assumptions for which each technique was developed. Considering these two factors, these traditional tests of CSR are not suitable in this scenario because the same distance between two RACs in the database (or two points in a simulation) could mean very different things depending on the local intensity [38-41]. In other words, two features in close proximity in an area with higher local intensity (more points per unit area) may not suggest a pattern, whereas the same observation in a region of low local intensity may provide evidence of clustering.

\section{Testing for CSR Adjusted for Spatial Inhomogeneity}

Based upon the results of power testing using traditional CSR techniques applied to shoe-specific contact-area modified simulations, it was prudent to evaluate the performance of spatial randomness approaches that are adjusted for inhomogeneity. As previously dis- 
cussed, the second-order statistic Ripley's K was selected for this study given its wide application in various disciplines utilizing spatial statistics. Given that this method requires local intensity estimation with window-specific edge correction, its likelihood of being fit-forpurpose was initially assessed using a subset of four shoes with a relatively large number of RACs $(83,90,101,114)$. Note that the average number of RACs per shoe is approximately $56(72,306 / 1,300)$, so the outcome from this small proof-of-concept study may not reflect the results likely for shoes with fewer RACs.

Figure 5 displays the Ripley's K results for four shoe-specific simulations and their associated empirically observed RACs; this analysis was conducted across 40 different radii ranging between 0-1,000px (0.0 - 41.6mm) and incremented by 25px (or approximately $1 \mathrm{~mm})$. As previously discussed, traditional interpretation of this test involves comparing K-distributions obtained from empirical data to $95 \%$ confidence envelopes derived from random simulations of the same number of observations in the same study region. Using this frequentist approach, if the observed accidentals on each shoe fall within the limits of the random realizations then adherence to CSR would be concluded at all distances. However, when likewise considering the confidence ranges of alternatives (clustering and dispersion) there are large areas of overlap between all three pattern types. This observation is alarming given that this second step is rarely taken in practice (in fact, the authors could not find a paper in which this approach was utilized). When considering all available simulations (null and alternatives), it becomes impossible to classify the structure of the empirical data. As an aside, these results likewise provide additional evidence that the dispersed simulations are not sufficiently dispersed for all shoes as near complete overlap exists for the Poisson and dispersed means and confidence bands.

In addition to the traditional frequentist interpretation of Ripley's $\mathrm{K}$ distributions, a Bayesian approach was undertaken in hopes of improving separation between distributions and offering more information for characterization of empirical RAC patterns with respect to randomness. Each simulated distribution was compared against the others in order to compute three score-based likelihood ratio (SLR) distributions informed by the empiricallycomputed Ripley's K value at each radius. Figure 6 exhibits the computed Ripley's K SLRs for each of the three inter-distribution comparisons across all radii evaluated: (i.) Poisson vs. 

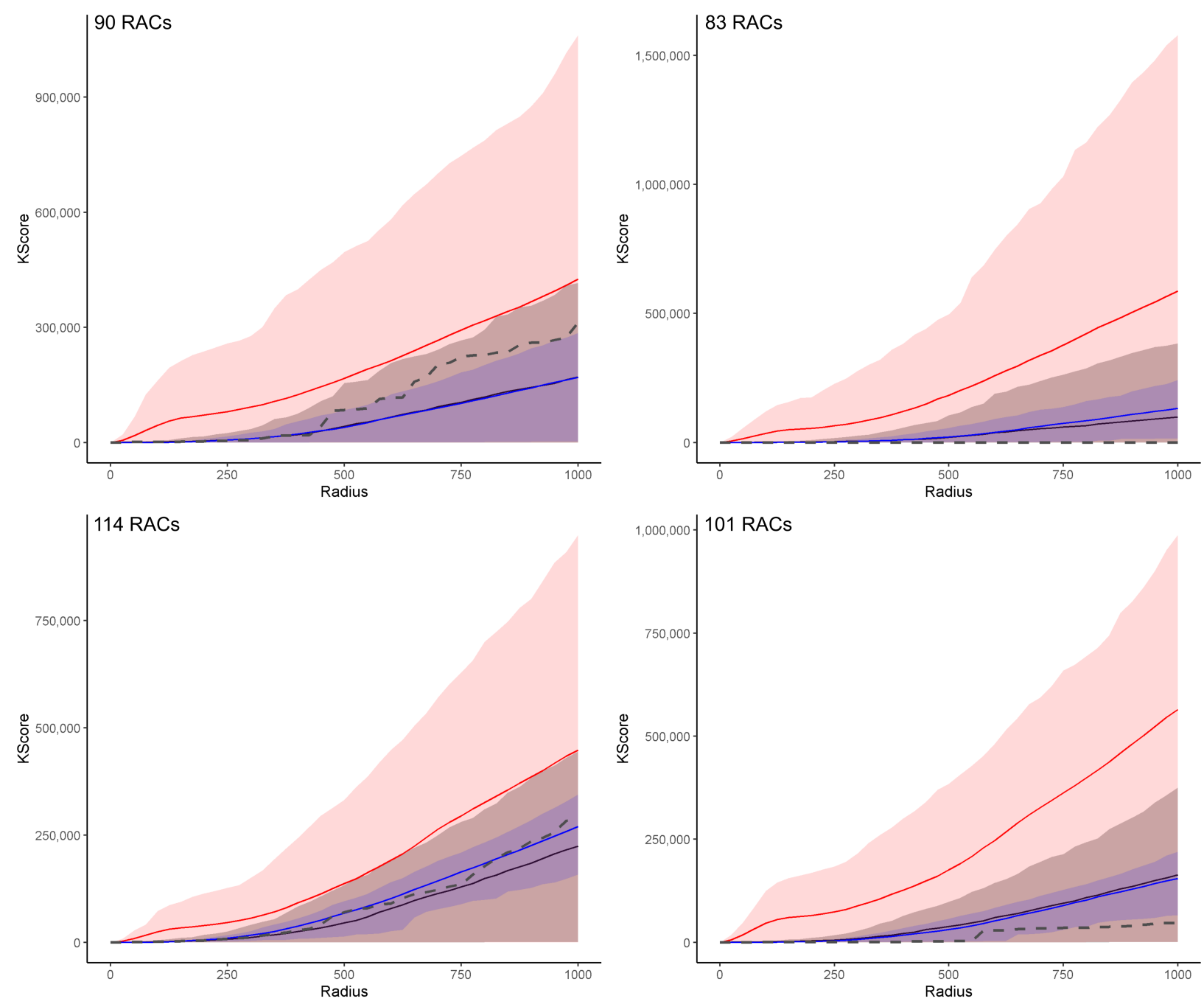

Figure 5: Ripley's K results for four shoes across various radii $(t=0-41.6 \mathrm{~mm})$. The dashed lines on each plot represent the distribution of $\mathrm{K}$ values for the empirically observed RACs on the given shoe. In addition, the mean lines (solid lines) and confidence bands (shaded regions) are provided for 500 simulations of Poisson (gray), clustered (red), and dispersed (blue) point processes generated on shoe-specific contact-area modified imagery.

clustered $(\mathrm{P} / \mathrm{C})$ represented by the red line, (ii.) Poisson vs. dispersed $(\mathrm{P} / \mathrm{D})$ represented by the blue line, and (iii.) clustered vs. dispersed (C/D) represented by the purple line. Based upon the pattern of each SLR plot, it is then possible to make inferences about the most probable pattern of RACs across various distances. For example, for the shoes with 83 and 101 RACs (the two right plots), it appears that there is evidence to support that the data are randomly distributed across all radii because the SLRs provided by the red and blue 

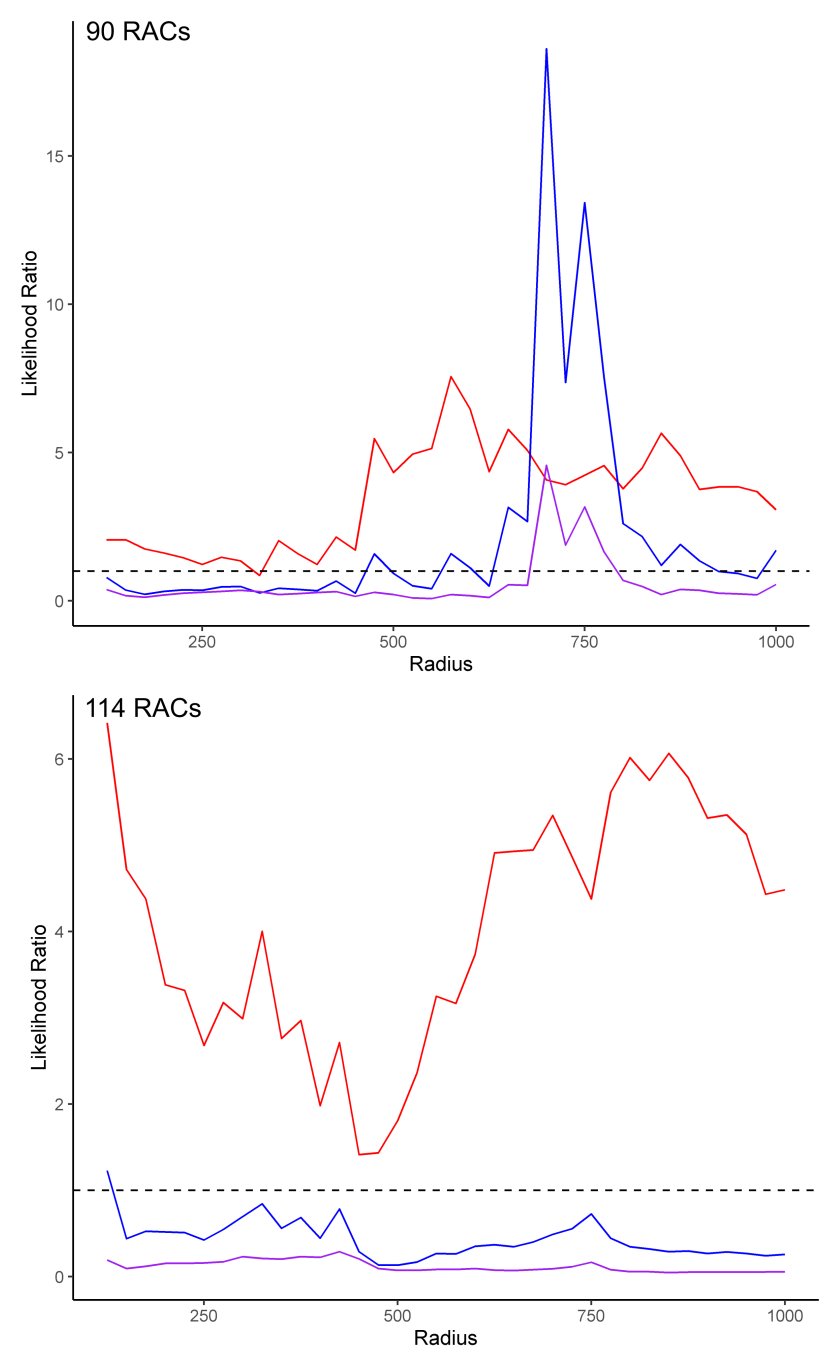

lines are larger in magnitude than that provided by the purple line (mean SLRs of 16-32 and 3.0-9.5 for red and blue versus 0.44-0.62 for purple). Conversely, based upon the bottom left plot of Figure 6, empirical RACs appear to be dispersed in space because there is more support for dispersion as compared to random (blue line) and clustered (purple line).
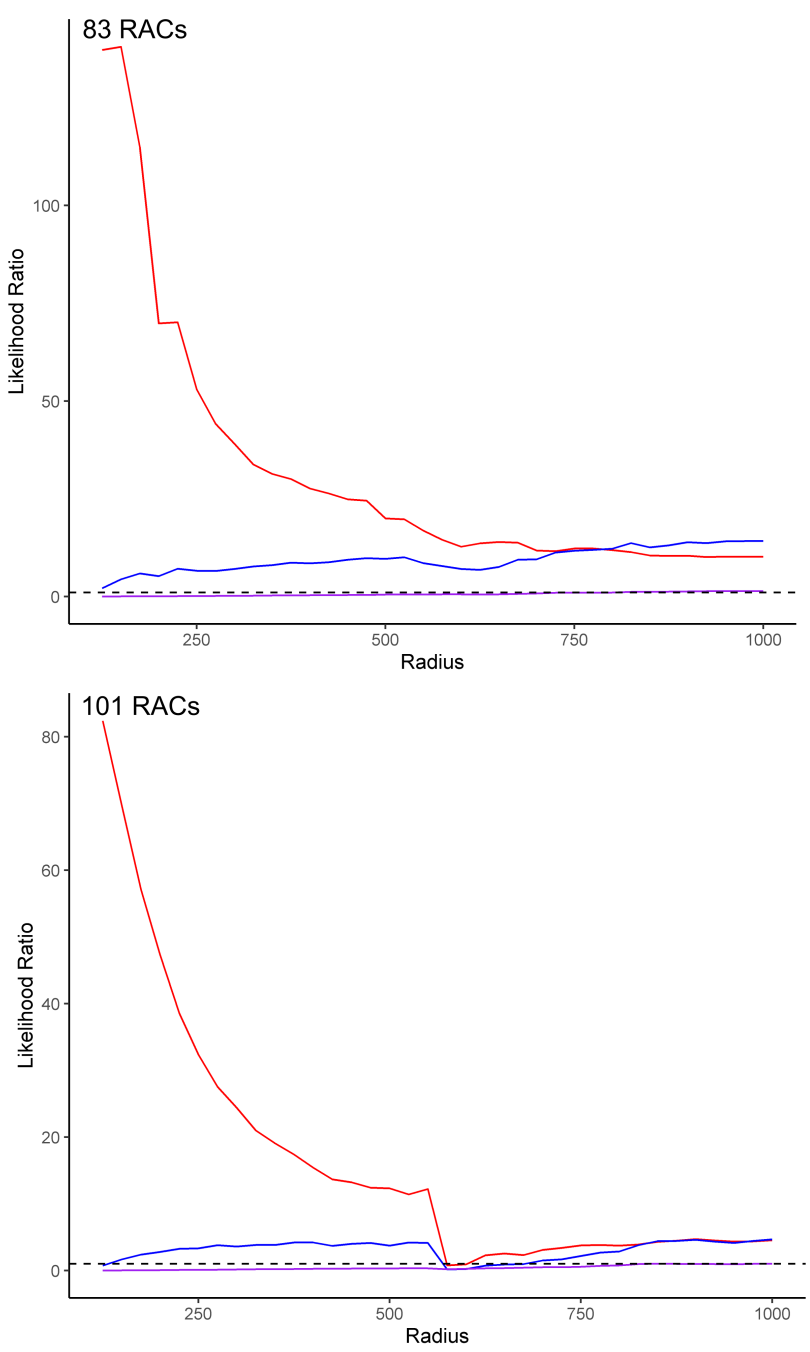

Figure 6: Score-based likelihood ratios (SLR) of Ripley's K results for four shoes across various radii $(t=$ $0,25,50, \ldots 1,000 \mathrm{px})$. The dashed lines on each plot indicates $S L R=1$, or the point at which there is a lack of evidence supporting one distribution over another. Results are plotted for comparison between the Ripley's $\mathrm{K}$ distributions of 500 simulations of Poisson, clustered, and dispersed simulations. More specifically, the red line represents the SLR for Poisson versus clustered, the blue line represents the SLR for Poisson versus dispersed, and the purple line represents the SLR for clustered versus dispersed. 
comes apparent that the relatively low population density for shoe-specfic point simulations will likely be a limiting factor for the utility of this technique for characterizing the spatial distribution of RACs, at least on the shoe-specific level. For the four outsoles assessed, each containing an above average number of RACs, the sample intensity ranges from $1.4 \times 10^{-5}$ to $1.8 \times 10^{-5}$ points per pixel (this equates to approximately $5.8 \times 10^{-7}$ to $7.5 \times 10^{-7}$ features per $\mathrm{mm})$. Given that Ripley's K is essentially a multi-distance measure of the expected number of points within a specified radius, this small spatial density can yield great variability in results, even for points with the same underlying distribution. As an example, consider the three clustered spatial distributions in Figure 7. All three squares are the same size, with the same number of points (10) and the same number of clusters (3), thus yielding the same intensity $(\lambda)$. Furthermore, each cluster is replicated exactly in all squares, with the only difference being the inter-cluster spacing. However, given the large spatial region in comparison to the number of points, the clusters can fall in a variety of positions with respect to each other.

Accordingly, the resulting values of $\mathrm{K}$ will be highly variable, with square A being large and square $\mathrm{C}$ exhibiting small values (with $\mathrm{B}$ be somewhere in between), despite the fact that each distribution was simulated using the same clustering criteria. This same trend is true on a large scale when considering the RACs in this study, exhibiting very low intensity values on a shoe-specific scale. Even when keeping simulation criteria consistent, there is great variability in the position of simulated RACs on outsoles. Accordingly, there is great variation in the values of Ripley's $\mathrm{K}$ within each distribution type, as exhibited by the near complete overlap in confidence bands for Poisson, clustered, and dispersed data in Figure 5. Moreover, since the four shoes selected were considered "best case scenarios" and contained well-over the mean number of features observed per shoe, it is highly likely that discrimination between pattern classes (Poisson, clustered, dispersed) will become increasingly more difficult as the number of accidentals decreases; as such, it was determined that given the sample and space of interest, Ripley's K was not "fit for purpose" in order to describe the underlying structure of RAC distributions on a shoe-specific level. 

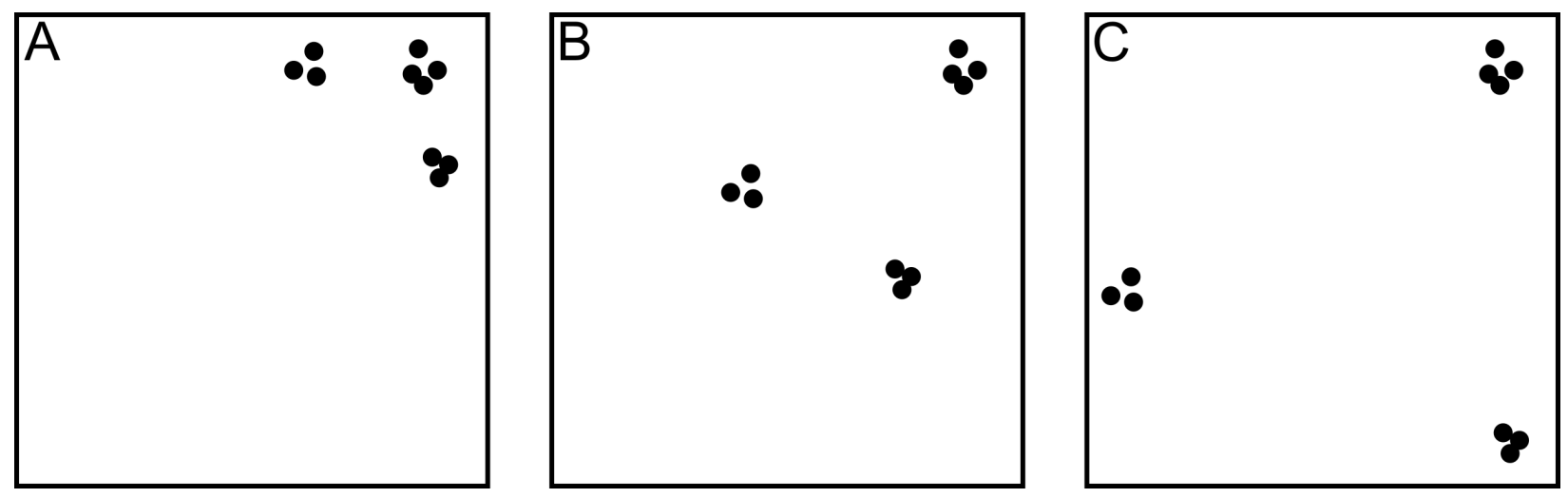

Figure 7: Illustration of the effect of low population intensity on Ripley's K variation. Each square contains a "simulated" distribution of 10 points in three distinct clusters, with the same sample intensity. However, given the relationships between each cluster, the Ripley's K associated with each square would be very different: high $\mathrm{K}$ for square $\mathrm{A}$, moderate $\mathrm{K}$ for square $\mathrm{B}$, and low $\mathrm{K}$ for square $\mathrm{C}$.

\section{Global Evaluation of Spatial Autocorrelation}

After sufficiently exploring tests of randomness (traditionally the first step in any spatial data analysis), additional assessments were conducted in an effort to characterize and describe the spatial distribution of RACs on outsoles contained within the WVU database. Namely, an evaluation of global autocorrelation of "observed residuals" on RAC counts was conducted using Moran's I, with the Poisson simulations serving as the predicted values against which other datasets were compared. This analysis was conducted in three phases in order to inform Type I error rate (Poisson versus Poisson), power (Poisson versus alternatives), and RAC patterns (Poisson versus empirical) and the results are presented in Table 7. Two of the sets of comparisons resulted in $0 \%$ departure from the expected value of Moran's I. More specifically, there was no significant global correlation detected for Poisson versus Poisson comparisons, thereby yielding a 0\% type I error rate. Given that this analysis was conducted on a small subset of heatmaps (10 pairwise compared resulting in 45 tests), these results suggest an acceptable, but in practicality likely non-zero, false rejection rate. In addition, an evaluation of the observed residuals for dispersed simulations versus the null revealed that none of the 100 comparisons exhibited significant spatial association. When considering previously obtained results for the dispersed alternatives, this observation of lack of spatial correlation provides further evidence that the dispersion factor of $85 \mathrm{px}(3.5 \mathrm{~mm})$ 
does not yield a sufficiently large effect size to be detected.

Table 7: Results of global assessment of spatial autocorrelation on observed residuals. For this study, the expected value of Moran's I is $-0.0011(-1 /(987-1))$, with values significantly larger suggesting positive correlation and values significantly smaller indicating negative correlation.

\begin{tabular}{|c|c|c|c|c|}
\hline Assessment & $\begin{array}{c}\text { Observed/Predicted } \\
\text { Distributions }\end{array}$ & $\begin{array}{c}\text { Total Number } \\
\text { of Comparisons }\end{array}$ & $\begin{array}{c}\text { Mean ( } \pm \text { sd) } \\
\text { Moran's I }\end{array}$ & $\begin{array}{c}\text { Percentage (Type) } \\
\text { of Global Correlation }\end{array}$ \\
\hline Type I Error & Poisson/Poisson & 45 & $-0.0012 \pm 0.0012$ & 0 (N/A) \\
\hline Power & Clustered/Poisson & 100 & $0.0032 \pm 0.0013$ & 87 (Positive) \\
& Dispersed/Poisson & 100 & $-0.0004 \pm 0.0019$ & $0(\mathrm{~N} / \mathrm{A})$ \\
\hline $\begin{array}{c}\text { RAC Count } \\
\text { Residuals }\end{array}$ & Empirical/Poisson & 100 & $0.1782 \pm 0.0030$ & 100 (Positive) \\
\hline
\end{tabular}

On the other hand, a global positive correlation was detected with $87 \%$ power, fortuitously exceeding the ideal threshold of $80 \%$, for the clustered in bin simulations, which exhibited a mean Moran's I value of $0.0032 \pm 0.0013$ (Table 7). Moreover, the evaluation of empirical versus Poisson distributions resulted in $100 \%$ identification of global spatial autocorrelation with a mean Moran's I more than 50 times higher than those yielded for the clustered alternatives at $0.1782 \pm 0.0030$. This outcome provides strong evidence for the true presence of a positive relationship between neighbors (wherein values near each other are similar), as illustrated in Figure 8 by the presence of distinct groups of off-zero residuals. Notably, in the ball of the toe the empirical data possess more RACs than predicted and conversely in the arch area and the edges of the toe and heel the observed RACs were fewer than expected based upon the Poisson simulations (both adjusted for bin partiality).

Based upon the global Moran's I and the visual examination of the observed residuals as a function of location, it is clear that the random simulations do not sufficiently account for the factors that influence RAC acquisition, despite the contact area modifications. One of the factors that is likely to affect feature development is degree of wear. Accordingly, in order to determine whether wear impacted the presence of RACs on an outsole a chisquare test of independence [48] was conducted and yielded a global result of significant dependence $\left(p=2.34 \times 10^{-13}\right)$ between overall degree of wear (light, moderate, high) and the number of accidentals visualized (1-25, 26-50, 51-76, 76-100, 101-150, 151+). Using an adjusted Pearson residual post-hoc test with a Bonferroni correction [49, 50], three pairwise 


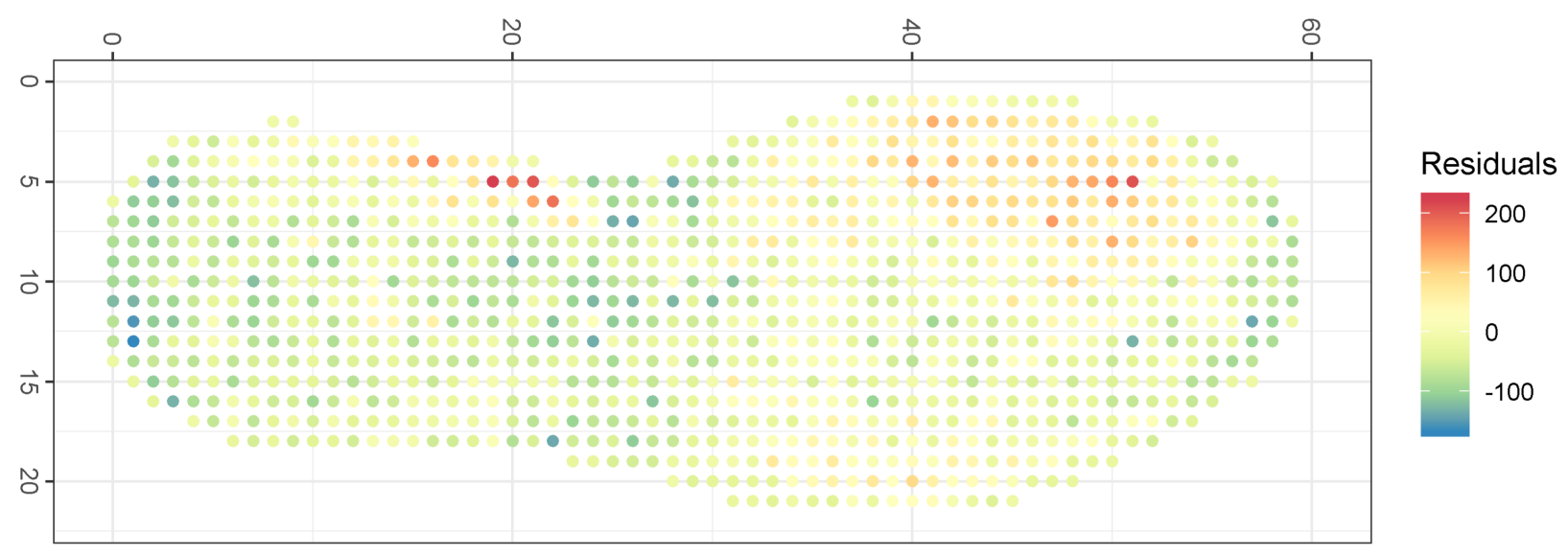

Figure 8: Observed residuals (color) for the empirical RAC modified counts versus the modified counts from a randomly selected Poisson realization (each computed according to Eq. 16). Global Moran's I indicated significant positive autocorrelation indicating that observed residuals were clustered in space. Note that feature counts for both the empirical data and Poisson realizations were modified to account for tread contact and bin partiality, according to Eq. 16.

comparisons exhibited evidence of dependence. More specifically, outsoles with light wear overall contained fewer RACs $(1-25)\left(p=1.75 \times 10^{-07}\right)$ and the opposite was observed for shoes with high wear of the sole material $\left(p=4.12 \times 10^{-12}\right)$. In addition, more shoes contained many RACs (151+) for heavily worn outsoles than would be observed if the two factors were independent of one another $\left(p=8.07 \times 10^{-08}\right)$. Therefore, based upon a global assessment of wear for each outsole, more wear intuitively results in more RACs acquired and thus observed and vice versa for less wear. Furthermore, this outcome may help to describe the observations in Figure 8 wherein little to no pressure is expected in the arch area (and thus fewer features are observed than expected in this region) and conversely more wear occurs on the ball of the toe and more RACs than predicted are present empirically.

\section{Local Indicators of Spatial Autocorrelation}

Following global assessment of spatial autocorrelation, a localized evaluation of Moran's I was conducted in order to determine whether associations existed within smaller neighborhoods. Local analysis of spatial association on "observed residuals," at 6 distances ranging from approximately 17 - 104mm, revealed significant positive correlation (similar values clustered together) in various outsole regions, as illustrated in Figure 9 based on comparisons 
between the empirical dataset and 10,000 Poisson realizations. Furthermore, the regions that exhibited residuals that deviated from zero for the global analysis (Figure 8) are the same regions exhibiting significant positive autocorrelation in a large fraction of all comparisons. Again, the ball of the toe contained more features than expected under randomness, while the arch and the edges of the heel and toe contain fewer RACs than predicted. Another important observation is that the locations of significant results, and the overall fraction of significant local correlation, is relatively stable across the neighborhood sizes specified in the weight matrix during computation of Moran's I, which range from $400 \mathrm{px}$ or $16.7 \mathrm{~mm}$ (the average radius of a lug) to $2500 \mathrm{px}$ or $104 \mathrm{~mm}$ (the distance across the widest part of the toe on the standard outsole). As illustrated in Figure 9, the distribution of color is relatively consistent in the aforementioned clusters of similar values (red cells), with increasing neighborhood sizes revealing some additional regions of local correlation (green-yellow color) in a fraction of comparisons.

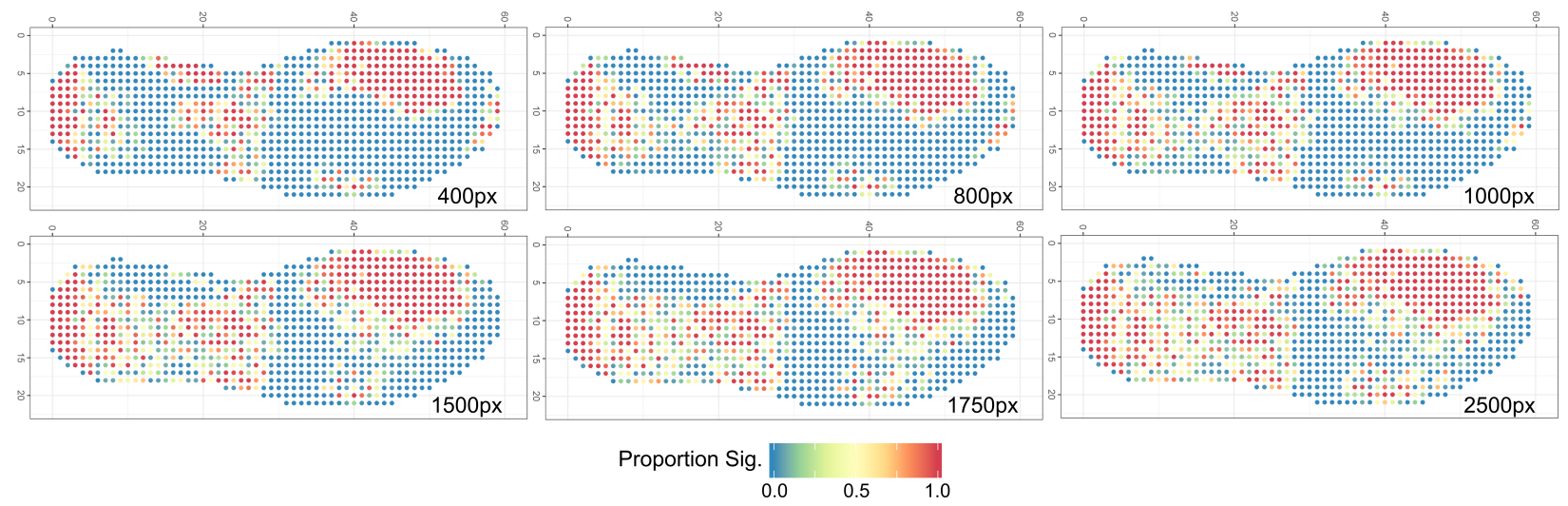

Figure 9: Fraction of empirical versus 10,000 Poisson observed residuals exhibiting significant local Moran's I results (color).

Collectively, the results from the global and local analysis of spatial autocorrelation of "observed residuals" computed through comparison of modified RAC counts between empirically observed and random predicted data indicate that overall the Poisson simulations do not adequately describe the factors which impact the distribution of accidentals on outsoles. Although the simulations accounted for shoe-specific tread areas, through utilization of a binary contact map, significant global and local positive correlation remains in the residuals. 
Based upon anecdotal observations, localized patterns of wear and pressure may account for these differences. In fact the areas exhibiting high proportions of significant autocorrelation in Figure 9 are in alignment with the maps of walking pressure distributions on outsoles collected by Liu et al. (2019) [51]. For example, Liu et al. (2019) [51] found that high pressure was placed on the ball of the toe and throughout the lower and lateral heel; this pattern aligns with results of the Moran's I analysis in this study. As such, it would be beneficial to evaluate whether characterization of wear across an outsole and subsequent modification of the results to consider this factor (just as contact area was included) would resolve the difference between the observed RACs and the random simulations.

\section{Conclusions}

The primary objective of this study was to evaluate the degree to which randomly acquired characteristics adhered to spatial randomness, after accounting for tread contact, by applying widely-used spatial statistics on a database of 72,306 features originating from 1,300 shoes. After a detailed examination of accidentals using a variety of techniques, three major conclusions can be gleaned. First, traditional approaches to testing for CSR are generally not suitable for the evaluation of RACs, owing to the inherent sample space heterogeneity. As previously discussed, the large majority of outsole surfaces are not uniform. Instead, shoes contain tread areas that contact the ground during use as well as raised areas between elements that would only reveal RACs in 3-dimensional impressions, which were not evaluated as part of this research. Accordingly, when specifically assessing 2-dimensional prints, the major CSR assumption of homogeneity is violated. Although the current study attempted to account for this violation by creating contact-modified imagery for each shoe, and associated Poisson simulations to represent a random distribution of RACs on tread areas, the power of traditional tests of randomness was much lower than ideal (maximum power of $65 \%$, with the majority of tests exhibiting a true rejection rate below 15\%). Therefore, traditional metrics for evaluating CSR are not fit-for-purpose for the analysis of RACs on outsoles because they are not sufficiently robust to violations of the homogeneity assumption, even after adjusting theoretical critical values using empirically derived null distributions.

Second, tests of spatial randomness adjusted for inhomogeneity, thereby relaxing the 
assumptions for CSR, contain limited utility for shoe-specific evaluations of randomness. Given the relative "rarity" of features on tread areas, as supported by the minute intensity estimates (on the magnitude of $10^{-7}$ points per $\mathrm{mm}$ ), there is likely insufficient information to obtain a reliable decision regarding adherence to randomness versus clustered or dispersed alternatives. This conclusion is revealed by the large variability in scores for each of the simulated RAC realizations (Figure 5), and thus discrimination between Poisson and alternative distributions is problematic. However, this study did not evaluate this approach on the heatmap-level (agglomerating all RACs), and can therefore not comment on utility in this context. Evaluation of database-wide RAC distributions using these approaches would require complex mappings of local intensity across the entire database, while accounting for shoe-specific outsole factors such as contact area.

Lastly, and potentially most importantly, the results from this study indicated that tread design alone did not sufficiently describe the spatial distribution of acquired features. Moreover, all areas of contact were not created equal and therefore equal degrees of contact did not necessarily yield similar frequencies of observed features. More specifically, as compared to the Poisson null realizations, observed residuals of empirical data resulted in significant global and local spatial correlation. Therefore, the Poisson random simulations did not adequately represent the distribution of observed features, suggesting that a significant latent explanatory factor remained that must be identified and incorporated. Based upon the locations of significant local association as well as an understanding of the physical process causing the development of RACs on outsoles, wear is one such variable that should be explored in more detail. Analysis of localized regions of outsole wear may provide information not only about tread contact, but also the dynamic process of gait and pressure that occurs when using a shoe, thus representing an important intersection of the dynamic process of RAC formation. As such, further research is required to determine the degree to which other readily observable outsole factors, that could be transferred to crime scene impressions and thus characterized as part of the analysis process, affect the distribution of RACs.

When considering the above conclusions, it is also important to address several studyrelated factors associated with observed results. First, the contact maps utilized to describe the positions on which RACs could occur were created automatically, using a series of mor- 
phological operators to segment outsole tread elements on exemplar impressions. While every effort was taken to ensure a reliable representation of the tread pattern, the contact maps are not perfect reproductions of the tread pattern. Thus, a more supervised process may yield more precise results. For example, using a high-quality outsole scanner and then manually adjusting the resulting binary images, may slightly increase the fidelity of the contact maps; however, it is unclear whether this expensive and timely approach would yield significant, worth-while improvements. In addition, this study utilized a warp process to normalize all shoes, and their associated RACs and contact maps, to a standardized heatmap outsole. While this process was necessary to increase the sample size of the database, this step may constrain the practical applicability of the results given the change in appearance of tread elements with respect to the original outsole. The only way to overcome this limitation would require an exorbitantly large database containing replicates of a wide variety of shoes with the same class characteristics (e.g., make, model, and size).

Furthermore, one of the limitations specifically related to the simulation of alternative distributions that must be acknowledged. As previously discussed, the dispersed simulations used in this study were not sufficiently dispersed to detect departures from randomness in most cases. In an effort to maintain consistency between outsoles, a single dispersion factor (85px or $3.5 \mathrm{~mm}$ ) was selected for all shoes based upon those outsoles in the database that contained the most features and/or the least amount of contact. In other words, given that these simulations were constrained by the number of RACs that could be projected onto a given area while still maintaining the distance between all points, this minimum dispersion factor was selected and applied to all simulations. Unfortunately, this led to many simulations being under-dispersed for a number of shoes and thus indistinguishable from CSR. While more appropriate realizations are currently being produced, the authors do not believe that the under-dispersion significantly impacted the conclusions from this study regarding the applicability of CSR approaches, given that the power was likewise inadequate for the clustered alternatives.

Despite these limitations, this work constitutes an in-depth examination of the distribution of RACs with regard to spatial randomness using a large-scale database of features identified on a variety of outsoles and appropriately modified for tread design. Moreover, 
this investigation was specifically targeted to determine the degree to which accidentals adhered to CSR, given the embedded and practically logical assumption of randomness as a function of the stochastic nature with which these features develop. Specific outcomes from this study suggest that classical testing approaches for evaluation of CSR are underpowered for this type of data and that more advanced and holistic techniques (e.g., modeling) are likely required and should be investigated moving forward. 


\section{References}

[1] W. J. Bodziak, Footwear Impression Evidence: Detection, Recovery, and Examination, CRC Press, 2000.

[2] M. J. Cassidy, Footwear Identification, Canadian Government Printing Centre, 1980.

[3] T. Fruchtenicht, W. Herzig, R. Blackledge, The discrimination of two-dimensional military boot impression based wear patterns, Science and Justice 42 (2002) 97-104.

[4] W. J. Bodziak, L. Hammer, G. Johnson, R. Schenck, Determining the significance of outsole wear characteristics during the forensic examination of footwear impression evidence, Journal of Forensic Identification 62(3) (2012) 254-278.

[5] T. Hannigan, L. Fleury, R. Reilly, B. O’Mullane, P. de Chazal, Survey of 1276 shoeprint impressions and development of an automatic shoeprint pattern matching facility, Science and Justice 46 (2006) 79-89.

[6] S. Parent, The significance of class associations in footwear comparisons, Impression and Pattern Evidence Symposium (2010).

[7] S. Gross, D. Jeppesen, C. Neumann, The variability and significance of class characterisitcs in footwear impressions, Journal of Forensic Identification 63(3) (2013) 332-351.

[8] SWGTREAD, Range of conclusions for footwear and tire impression examinations, (https://www.nist.gov/sites/default/files/documents/2016/10/26/swgtread_ 10_range_of_conclusions_standard_for_footwear_and_tire_impression_ examinations_201303.pdf), 2013.

[9] W. J. Bodziak, Forensic Footwear Evidence, Taylor \& Francis Inc, 2016.

[10] T. W. Adair, J. Lemay, A. McDonald, R. Shaw, R. Tewes, The Mount Bierstadt study: An experiment in unique damage formation in footwear, Journal of Forensic Identification 57(2) (2007) 199-205.

[11] C. Hamburg, R. Banks, Evaluation of the random nature of acquired marks on footwear outsoles, Impression and Pattern Evidence Symposium, 2010. 
[12] N. Petraco, C. Gambino, T. Kubic, D. Olivio, N. Petraco, Statistical discrimination of footwear: A method for the comparison of accidentals on shoe outsoles inspired by facial recognition techniques, Journal of Forensic Sciences 55 (2010) 34-41.

[13] H. Wilson, Comparison of the individual characteristics in the outsoles of thirty-nine pairs of Adidas Supernova Classic shoes, Journal of Forensic Identification 62(3) (2012) $194-203$.

[14] J. Speir, N. Richetelli, M. Fagert, M. Hite, W. J. Bodziak, Technical note: Quantifying randomly acquired characteristics on outsoles in terms of shape and position, Forensic Science International 266 (2016) 399-411.

[15] N. Richetelli, W. Bodizak, J. Speir, Empirically observed and predicted estimates of chance association: Estimating the chance association of randomly acquired characteristics in footwear comparisons, Forensic Science International 302 (2019) 109833.

[16] R. Stone, Footwear examination: Mathematical probabilities of theoretical individual characteristics, Journal of Forensic Identification 56 (2006) 577-599.

[17] A. S. Fawcett, The role of the footmark examiner, Journal of the Forensic Science Society 10 (1970) 227-244.

[18] Y. Yekutieli, Y. Shor, S. Wiesner, T. Tsach, Expert Assisting Computerized System for Evaluating the Degree of Certainty in 2D Shoeprints, Technical Report, National Institute of Justice, 2012.

[19] P. J. Diggle, Statistical Analysis of Spatial and Spatio-Temporal Point Patterns, Taylor \& Francis Inc, 2013.

[20] N. A. C. Cressie, Statistics for Spatial Data, John Wiley \& Sons, Inc., 2015.

[21] A. Baddeley, E. Rubak, R. Turner, Spatial Point Patterns: Methodology and Applications with R, Apple Academic Press Inc., 2015.

[22] M. Mugglestone, E. Renshaw, Spectral tests of randomness for spatial point patterns, Environmental and Ecological Statistics 8 (2001) 237-251. 
[23] O. Schabenberger, C. Gotway, Statistical Methods for Spatial Data Analysis, Chapman \& Hall/CRC, 2005.

[24] T. Smith, Notebook on spatial data analysis, Online, 2020. https://www . seas . upenn. edu/ ese502/\#notebook.

[25] D. W. Goodall, A new method for the analysis of spatial point pattern by random pairing of quadrats, Vegetatio 29(2) (1974) 135-146.

[26] J. A. Ludwig, D. W. Goodall, A comparison of paired- with blocked- quadrat variance methods for the analysis of spatial pattern, Vegetatio 38 (1978) 49-59.

[27] G. W. Snedecor, W. G. Cochran, Statistical Methods, Iowa State University Press, 1989.

[28] C. Liu, A comparison of five distance-based methods for spatial pattern analysis, Journal of Vegetation Science 12(3) (2001) 411-416.

[29] D. Edbon, Statistics in Geography, Blackwell, 1985.

[30] P. Clark, F. C. Evans, Distance to nearest neighbour as a measure of spatial patterns in biological populations, Ecology 35 (1954) 445-453.

[31] P. Dixon, Nearest neighbor methods, Encyclopedia of Environmetrics 3 (2006).

[32] K. Donnelly, Simulation Studies in Archaeology, Cambridge University Press, pp. 91-95.

[33] J. H. Pollard, On distance estimators of density in randomly distributed forests, Biometrics 27 (1971) 991-1002.

[34] P. Ellis, The Essential Guide to Effect Sizes: Statistical Power, Meta-Analysis, and the Interpretaion of Research Results, Cambridge University Press, 2010.

[35] J. Lee, D. W. Wong, Statistical Analysis with ArcView GIS, John Wiley, 2001.

[36] A. C. Davison, D. V. Hinkley, Bootstrap methods and their Applications, Cambridge University Press, 1997. 
[37] E. C. Pielou, The use of point to plant distances in the study of the pattern of plant populations, Journal of Ecology 49 (1959) 255-269.

[38] M. Kulldorff, Tests of spatial randomness adjusted for an inhomogeneity, Journal of the American Statistical Association 101(475) (2006) 1289-1305.

[39] A. Baddeley, J. Moller, R. Waagepetersen, Non- and semi-parametric estimation of interaction in inhomogeneous point patterns, Statistica Neerlandica 54(3) (2000) 329350.

[40] C. Comas, M. Palahí, T. Pukkala, J. Mateu, Characterising forest spatial structure through inhomogeneous second order characteristics, Stochastic Environmental Research and Risk Assessment 23(3) (2008) 387-397.

[41] P. Henrys, P. Brown, Inference for clustered inhomogeneous spatial point processes, Biometrics 65(2) (2009) 423-430.

[42] M. F. Goodchild, Spatial Autocorrelation, Geo Books, 1986.

[43] A. Getis, Handbook of applied spatial statistics: Spatial Autocorrelation, 2010.

[44] P. A. P. Moran, Notes on continuous stochastic phenomena, Biometrika 37 (1950) $17-23$.

[45] G. Resende, Spatial dimensions of economic growth in brazil, ISRN Economics 2013 (2013) 1-19.

[46] L. Anselin, Local indicators of spatial association - LISA, Geographical Analysis 27(2) (1995) 93-115.

[47] D. Murdoch, Y. Tsai, J. Adcock, P-values are random variables, The American Statistician 62(3) (2008) 242-245.

[48] M. McHugh, The Chi-square test of independence, Biochemica Medica 23(2) (2013) 143-149. 
[49] D. Sharpe, Your chi-square test is statistically significant: Now what?, Practical Assessment, Research \& Evaluation 20(8) (2015).

[50] Cornell Statistical Consulting Unit, Adjusted standardized residuals for interpreting contingency tables, StatNews 95 (2018).

[51] L. Liu, W. Wang, Y. Luo, Foreign object held in recessed areas of shoe outsole as an acquired characteristic in footwear examination: A preliminary study, Forensic Science International 304 (2019) 109949. 
4. Spatial Modeling of RACs as a Function of Outsole Factors: Part I 


\title{
Spatial Regression Modeling of Randomly Acquired Characteristics on Outsoles with Implications Regarding Weight of Evidence Determinations: Part I
}

\begin{abstract}
Keywords: Forensic Footwear, Randomly Acquired Characteristics, Weight of Evidence, Spatial Regression Modeling, Spatial Autocorrelation
\end{abstract}

\section{Introduction}

Serving as the cornerstone of forensic footwear analysis is the determination of origin for an evidence impression with respect to a suspect shoe as function of the observed agreement, or lack thereof, in patent class, subclass, and randomly acquired characteristics (RACs). Further informing this comparison between known and questioned sources is the perceived strength assigned to the identified similarities and/or dissimilarities between features, which is a function of their associated clarity, rarity, and discriminating potential. Given sufficient clarity and quality, the presence of RACs, which develop on outsoles as they are worn and are considered to be highly variable between individual shoes [1-11], forms the basis of source attribution.

When specifically considering the evidential weight of observed randomly acquired characteristics, two major factors are required to inform this value. First, the features of interest (RACs) must be sufficiently variable in position, shape, size, orientation, and geometric complexity, in order to minimize the probability of encountering two unrelated features that are confused as originating from the same source. Considerable research effort has been focused on characterizing this chance association using both theoretical and empirical means $[1-5,7,9,10]$, and these have been summarized in Chapter 2 of this document. Ultimately, multiple of these studies found similar magnitudes of chance agreement, despite differences in study design [3, 7, 10]. For example, Stone [3] computed probabilities for various theoretical accidentals as a function of both general shape and orientation (where applicable), and assuming a uniform chance of positional overlap across all possible locations 
on the outsole. Namely, Stone's work reported chance agreements of 1 in 384,000 and 1 in 3,070,000-24,600,000 for lines and irregular enclosures, respectively. As an extension, the work detailed in Chapter 2 of this document [10] characterized the stochastic overlap of empirically observed features as a function of general shape, visual similarity (which encapsulates orientation), and position. Figure 1 details the spatial distribution of the resulting chance associations [10]. The resulting median probability values for feature overlap ranged from 1 in 541,276 to 1 in 18,031,824 for linear and variable features, respectively [10], and are thus in alignment with those detailed by Stone [3].

However, visual inspection of the shape-specific distributions of chance associations illustrated in Figure 1 reveals that the probabilities seem to vary across the outsole. In other words, the chance that two unrelated RACs are confused as originating from the same shoe depends upon their spatial position. Based upon the factors considered in computation of these values, this observation could be a function of the accidentals' shapes, locations, or both. Given that shape classifications are expected to become less reliable as impression quality degrades (i.e., in a crime scene print) [10, 12], subsequent work focused on RAC spatial distributions as a function of feature location only. Moreover, since the probability of positional co-occurrence within each cell was dependent upon the number of shoes containing tread within that region, it was purported that localized degree of contact was an influential variable informing feature distributions.

In an effort to shed additional light on this hypothesis, a formal evaluation of randomness was conducted using traditional spatial statistics techniques in order to determine whether RACs adhered to randomness after accounting for tread contact. This work was formally detailed in Chapter 3 of this document and will only be reviewed briefly here. With the primary goal of performing a detailed evaluation of RAC distributions as a function of tread design, this research effort utilized a combination of contact-area modified imagery, synthetic Poisson (and alternative) point processes, and spatial statistical approaches aimed at informing the community regarding assumptions related to randomness. Based upon the results of this study, there were three important lessons learned that must be considered to properly evaluate spatial distributions of acquired features moving forward.

First, traditional methods for evaluating adherence to complete spatial randomness (CSR) 

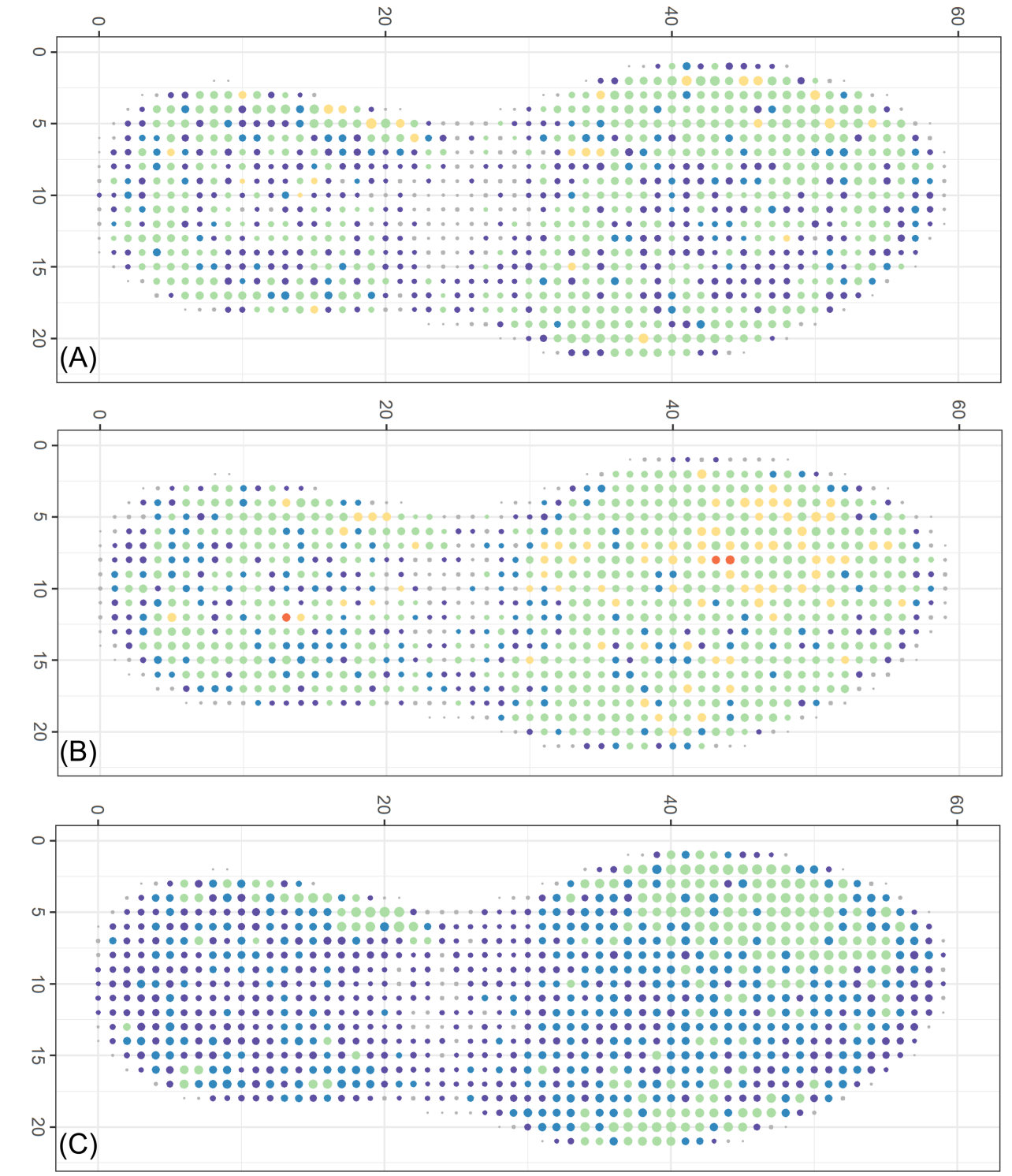

Chance

- N/A

- 1 in $10,000-1$ in 50,000

1 in $50,000-1$ in 100,000

- 1 in 100,000 - 1 in 500,000

- 1 in 500,000 to 1 in $1,000,000$

- 1 in $>1,000,000$

RACs

. 0

- 25

- 50

- 75

Figure 1: Chance association (color) as a function of RAC counts (point size) for linear (A), compact (B), and variable $(\mathrm{C})$ shaped features [10].

are unsuitable for the assessment of the spatial distribution of RACs. Given the inherent heterogeneity of the study region, likely due to the non-uniformity of outsole tread areas. As such, a more holistic technique, that comprehensively describes the data as opposed to reducing relationships to a test statistic, is necessary. Second, the results indicated that the spatial distribution of accidentals is not solely based upon tread contact, as initially hypothesized. This conclusion is supported through the evaluation of observed residuals, or 
the comparison of the empirically collected RACs versus the contact-area modified Poisson simulations, as detailed in Chapter 3 (Table 7). Third, equal contact between two locations does not suggest that similar numbers of RACs will be observed at each location, and rather there is local association in counts depending on the region of the outsole. As exhibited in Figures 8 and 9 of Chapter 3, there are regions of significant local positive associations, with the strongest effects observed in the ball of the toe, the arch, and the edge of the heel. Thus there remains a significant, unmodeled factor, which could be theoretical (spatial heterogeneity or interaction between neighboring cells) and/or practical (another characteristic of the outsole or acquisition process) and must therefore be evaluated.

When taken collectively, these conclusions indicate that a correlation exists between neighboring RAC counts in some regions of the empirical heatmap. For example, the arch area and edge of the heel exhibit numerous cells with significant local correlation because RAC counts are consistently over-predicted in this area, leading to clusters of negative residuals. Conversely, frequencies are under-estimated in the ball of the toe, yielding significant positive association between positive residual values. In addition, traditional tests and approaches of characterizing these underlying patterns are not suitable for examination of outsoles given the inherent violation of homogeneity in the study region. However, the above observation that RACs exhibit spatial correlation is illogical when considered practically based upon the process by which these features are acquired. In other words, it is unlikely that the presence of an accidental promotes or deters the development of another in that area. Instead, it is more feasible that specific conditions of the outsole or acquisition process introduce spatial association in various regions, thus imparting what appears to be a pattern in features. Therefore, in order to inform weight of evidence of RACs it is imperative to characterize which factors impact accidental distributions and to what extent spatial proximity and dependence of these factors influence these observations downstream.

Accordingly, the primary aim of this research is to simultaneously understand how one (or more) outsole-related variables, as well as the nature of the spatial relationship between these factors, impacts the observed spatial distribution of a collection of RACs from a large scale database of shoes. Spatial regression methods are particularly suited for this analysis because their principle function is to detect and model relationships between patterns in 
order to understand the processes responsible for any observed patterns that are unlikely to arise from random variation alone [13-20].

\section{Overview of Spatial Regression Modeling}

Spatial modeling techniques have historically been used to study economic systems. However, in the last several decades, these methods have garnered widespread implementation in a number of other scientific fields, owing to the fact that they are easily adapatable and robust for a multitude of data types [14, 20-22]. Furthermore, it is commonly observed that data collected from regions or points in space are rarely independent, and instead exhibit some spatial heterogeneity (inherent non-uniformity of tread contact) and/or correlation (significant positive correlation of observed residuals, as detailed in Chapter 3) [13-20, 22], and RACs appear to be no exception. Under these conditions, traditional approaches to data analysis are unsuitable and produce biased predictions or estimates of effects given the violation of critical assumptions $[13,14,19,20,23]$. As an alternative, spatial regressions are able to capture dependencies across space and simultaneously incorporate and evaluate other variables that are deemed important to characterizing a specific phenomenon (the spatial distribution of RACs for this study).

Therefore, one of the primary goals of spatial modeling is to understand the "data generating process," or the factors and relationships that yield an observed spatial distribution $[14,15,19]$. However, there are a variety of different models that incorporate various types of spatial structures/interactions between variables that could be used to characterize this process. Absent knowledge about this process, which is often the case in practical applications of these techniques, there are generally two approaches applied to determine which type of model is most suited to the data: bottom-up and top-down implementations [15, 19]. The bottom-up approach starts with the most conservative, parsimonious model (a traditional non-spatial regression) and subsequently moves up to more robust models that include additional potential sources of spatial correlation (spatial models that incorporate lags in the reponse and/or predictors) $[15,19,24]$. Alternatively, the top-down approach begins with the most robust model, which includes spatial contributions from both the response and the predictors, and then incrementally removes the interaction parameters $[15,19]$. When 
conducting initial data analysis, the bottom-up technique has been widely recommended and utilized by numerous authors in the field because the interative expansion of the standard regression with additional spatial lag variables more reliably reveals the true data-generating process and more clearly allows for the interpretation of main effects [15, 19, 24, 25].

Irrespective of the technique applied, spatial regression modeling produces four main deliverables that are instrumental in understanding the relationships and factors influencing a phenomenon of interest, and these are all useful for characterization of RAC distributions and subsequent consideration of weight of evidence of observations [13, 14, 17, 20]. First, the implementation of multiple model types (via bottom-up or top-down approaches) allows for the simultaneous consideration of multiple factors, spatial interactions, and scales to describe the underlying data generating process [13-17, 19, 20, 24]. Next, an analysis of the estimated coefficients allows for an interpretation of the magnitude and direction that each predictor has on the response $[14,15,17,19]$. Third, through the incorporation of weighted averages (or spatial lags) of predictors and/or responses, spatial regression methods are able to capture dependencies across spatial units, thus allowing for an assessment of the strength and direction of spatial correlation, both globally and locally $[14,15,17,19,24,26]$. Lastly, results from assessment of multiple neighborhood sizes yield an understanding of the connectivity structure underlying the phenomenon, wherein a determination of the distance or scale of correlation can be evaluated. Consequently, spatial regression approaches allow for a comprehensive understanding of the data-generating process via a detailed implementation and evaluation of various factors and spatial correlation structures, and neighborhood sizes $[14,17,24,26]$. For the purposes of this investigation (Part I), RAC counts will be evaluated as a function of contact area and including various types of spatial structures, but only for a fixed scale using a single neighborhood cut-off value.

Therefore, given the ready adaptability and flexibility of spatial regression techniques, coupled with the immense utility and information provided by the results, these methods were utilized to further understand the distributions of randomly acquired characteristics on outsoles. More specifically, in pursuit of determining the factors that impact the spatial distributions of RACs and characterizing their resulting effects, this work used a database of 72,306 RACs identified on 1,300 shoes to model spatial distributions of RACs as a function of 
tread contact area. Evaluation and ultimate selection of the optimal model will reveal relationships between accidentals and outsole contact as well as the associated spatial structure that influences the distribution of identified features on shoes downstream. Ultimately, the acquired knowledge about which factors introduce associations and the magnitude and nature of these interactions will allow for a more accurate and informed assessment of weight of evidence of observed accidentals depending on spatial location and local outsole conditions.

\section{Materials and Methods}

\section{Spatial Regression Modeling Preliminaries}

Prior to discussion of study-specific methods, this section serves to briefly describe the four Poisson regression techniques that were used to characterize RAC distributions as a function of outsole-related factors on both global and local scales. More specifically, these techniques allow for the incorporation of spatial effects in order to more accurately characterize a phenomenon of interest. In general, there are two types of spatial effects, autocorrelation and heterogeneity, and the presence of either can preclude utility of traditional analyses owing to violations of embedded assumptions, such as independence between observations $[14,15,27]$. Spatial autocorrelation arises when there is an association or interaction between variables (responses and/or predictors) within a local region [14, 15, 27]. On the other hand, spatial heteorogeneity occurs when parameters vary across space and thus relationships between variables are different depending on the region $[14,15,27]$. In conducting exploratory spatial analysis, it is therefore necessary to evaluate and account for both of these spatial factors in order to appropriately describe the data generating process.

As previously described, the bottom-up approach was selected for this study of RAC distributions and four regression approaches were applied; each is described in greater detail below. Investigation was initiated with a traditional non-spatial model. Since the response of interest was feature counts, a Poisson generalized linear model was employed. Subsequently, a spatial Auto-Poisson regression was used in an attempt to characterize endogenous interactions which occur when the outcome in a specific area is dependent on that of its neighbors $[14,15,18,19]$. Next, in order to characterize whether both endogenous and exogenous interactions exist, in which the observation at a given location is dependent on 
both the response and the predictor values of its neighbors, a spatial Poisson Durbin model was selected $[14,15,18,19]$. Lastly, to evaluate for the presence of spatial heterogeneity, a globally-weighted Poisson regression was used in order to assess local effects of predictors $[15,24,26]$.

\section{Non-Spatial Generalized Linear Model (GLM)}

Serving as a baseline model, the frequency of RACs in heatmap bins was modeled using a Poisson generalized linear regression. This global model does not consider the effect of spatial relationships between observations, and therefore contains an underlying assumption of independence. Given that the response of interest for this study was feature counts $(Y)$, a Poisson regression was selected to model the data according to Eq. 1 [15, 19, 24], where $X$ represents a matrix of predictor variables for each observation and $\beta$ represents a vector of modeled coefficients for each predictor.

$$
\begin{aligned}
Y & \sim \operatorname{Poisson}(\lambda) \\
\log (\lambda) & =\beta_{0}+X \beta+\epsilon
\end{aligned}
$$

Spatial Auto-Poisson Model (AP)

The spatial Auto-Poisson regression, a simple extension of the non-spatial Poisson GLM, accounts for spatial autocorrelation by incorporating a neighborhood weighted average of the observations as a predictor in the model $[14,15,19,20,24]$. In other words, this global approach characterizes the degree to which the response at one location is influenced by neighboring values of the same variable and thus evaluates endogenous interactions. In order to compute this weighted average, a weight matrix is required which defines the neighborhood structure of the data [13-15, 20]. Furthermore, a distance cut-off can be specified beyond which all weights are assigned a value of zero, thereby not imparting an effect on the cell of interest. For the purposes of this study, a row-normalized distance-based weight matrix $(W)$ was used wherein the weight $\left(w_{i j}\right)$ between two neighboring cells ( $i$ and $j$ ) was the inverse Euclidean distance between their centroids divided by the sum of the row.

Since the spatial AP model allows for spatial association between neighboring response values (RAC counts), a response spatial lag vector is included as a predictor $\left(W_{y}\right)$ and its 
coefficient $(\rho)$ is likewise estimated and evaluated for significance. The value of $W_{y}$ at each location $i$ is computed according to Eq. 2, where $y_{i}$ is the response value at location $i$ and $n$ is the total number of locations (987 for this study). Thus, after constructing the weight matrix and calculating the associated lag parameter on the response, a spatial Auto-Poisson model can be estimated according to Eq. 3 [14, 15, 19, 20, 24].

$$
\begin{aligned}
W_{y i} & =\frac{\sum_{j \neq i}^{n} w_{i j} y_{i}}{\sum_{j \neq i}^{n} w_{i j}} \\
\log (\lambda) & =\beta_{0}+X \beta+\rho W_{y}+\epsilon
\end{aligned}
$$

\section{Spatial Poisson Durbin Model}

Following estimation of the non-spatial GLM and the spatial AP models, representing more parsimonious approaches, a spatial Durbin regression can be applied to incorporate spatial lags in both the response (as in the AP regression) as well as the predictors [13$15,19,20]$. This is the most robust global model because it accounts for the potential presence of both endogeneous and exogenous interactions and therefore can produce unbiased coefficient estimates even in the presence of spatially autocorrelated errors [15, 19]. In order to incorporate exogenous interactions, spatial lags must be computed for any predictor(s) of interest (where $k$ represents the number of included factors) thereby allowing for neighboring values of these factors to affect estimates of the response at each location. The spatial lag for each independent variable $(W X)$ is computed by multiplying the weight matrix by the $n$-length vector of the values at each location and is included as an additional predictor in the model and a coefficient is estimated for each (resulting in $\theta$, a $k$-length vector). Using the same weight matrix $(W)$ and response association $\left(W_{y}\right)$ as outlined for the spatial AP regression and including the lag parameter for the predictor(s) of interest, a spatial Durbin model can be estimated according to Eq. 4.

$$
\log (\lambda)=\beta_{0}+X \beta+\rho W_{y}+\theta W X+\epsilon
$$


Spatial Geographically-Weighted Poisson Model (GWPR)

Although all of the above methods incorporate spatial lag parameters that model autocorrelation, they fail to allow for the presence of spatial heterogenity owing to their global nature (i.e., a single model is estimated to represent all of the data). To properly account for any spatial instability, it is thus required that local modeling be conducted, thereby allowing for variables to impart different effects on the response at different locations [14, 15, 27]. Geographically-weighted Poisson regression is a local form of the global Poisson regression and estimates a model for each cell's $(i)$ centroid location $\left(u_{i}, v_{i}\right)$ as a function of the predictors of neighboring areas, as illustrated in Eq. 5 [24, 26, 27]. More specifically, model coefficients at each location are estimated using iteratively reweighted least squares, accounting for neighboring values of both the responses and predictors according to Eq. 6 [27], where $X$ represents the predictor variable matrix, $W_{i}$ represents the diagonal of the weight matrix $(W)$ for cell $i$, and $Y$ represents the vector of responses. Therefore, this approach incorporates heterogeneity in both the response and predictor variables by estimating a model at each location of interest, and accordingly allows for an evaluation of the effect of each factor throughout a spatial region.

$$
\begin{aligned}
\log \left(\lambda_{i}\right) & =\beta_{0}\left(u_{i}, v_{i}\right)+X \beta\left(u_{i}, v_{i}\right)+\epsilon \\
\hat{\beta}_{i} & =\left(X^{T} W_{i} X\right)^{-1} X^{T} W_{i} Y
\end{aligned}
$$

\section{Database and Pre-Processing}

The Speir Research Group at West Virginia University (WVU) houses a collection of 1,300 shoes for analysis, varying in both class and subclass characteristics (manufacturer, style, size, degree of wear, etc.) [28]. Each of the aforementioned outsoles were examined for the presence of accidentals, and in total 72,306 RACs were identified, characterized, and localized via a semi-automated image processing chain [28]. Subsequently, RACs were remapped onto a standard outsole in order to allow for increased power of analysis, and finally segmented into 987 bins to create a frequency heatmap [10, 28]. This heatmap is publicly available at http://www. 4n6chemometrics.com/database/ and provides information about RAC frequencies, number of shoes contributing RACs, and probabilities of encountering 109 
RACs at each location in the database, as previously detailed in [10, 28].

\section{Evaluation of RAC Distribution as a Function of Contact Area}

Given that the database contains a variety of shoes, with different class and subclass characteristics, there is likewise considerable variation in the outsole geometric designs. Moreover, the large majority of outsoles contained in the database are not uniform in nature, but instead possess tread elements. Since this research only considers two-dimensional impressions, the observable RAC locations were constrained to those that fell on tread contact areas, and generally excluded the raised voids between these elements. As such, in order to describe the positions on each shoe that would have the potential to develop characteristics of use that could be visualized in a two-dimensional impression, binary contact maps were created, as previously detailed in Chapter 3. Subsequently, the amount of contact within each bin was determined for each shoe and then summed to obtain the total contact area at each location.

Although previous results indicated that contact alone was not the only factor impacting RAC count, as evidenced by the lack of fit between the empirical and Poisson simulated distributions detailed in Chapter 3, these methods did not account for the presence of spatial effects (i.e., spatial autocorrelation between locations and/or spatial heterogeneity of variables) $[14,15,27]$. Therefore, in order to capture and describe any spatial effects underlying RAC distributions as a function of tread design, spatial regression modeling was implemented. For each model, the number of RACs at each heatmap location was the observed response variable $(R)$, while the average contact area $(C$, or total contact divided by the number of shoes) was used as a predictor. In addition, the bin partiality (percentage of the bin within the heatmap frame) was included as an offset variable $(B)$ to prevent confounding as a function of unequal cell size. Four models were constructed to evaluate the distribution of accidentals as a function of contact area and spatial effects (Eqs. 7-10), following the previously outlined approaches. For this investigation of the distribution of 72,306 RACs versus contact area extracted from 1,300 shoes, neighborhood cut-off values of 800px (approximately $34 \mathrm{~mm}$ ) were used in the weight matrix of all spatial models; this value was selected because it represents the upper limit of lug size (mean $+2 \mathrm{SD})$ within the WVU database as previously outlined in Chapter 2. 


$$
\begin{aligned}
& \log (R)=\beta_{0}+\beta_{1} C+\beta_{2} \log (B) \\
& \log (R)=\beta_{0}+\beta_{1} C+\beta_{2} \log (B)+\rho W_{R} \\
& \log (R)=\beta_{0}+\beta_{1} C+\beta_{2} \log (B)+\rho W_{R}+\theta W C \\
& \log (R)=\beta_{0}\left(u_{i}, v_{i}\right)+\beta_{1} C\left(u_{i}, v_{i}\right)+\beta_{2} \log \left(B\left(u_{i}, v_{i}\right)\right)
\end{aligned}
$$

\section{Results and Discussion}

\section{Database and Pre-Processing}

After discretization of all 72,306 RACs into 987 heatmap cells, there was an average of $73 \pm 36$ accidentals in each location, with a minimum of 0 and a maximum of 189 . Figure 2 illustrates the spatial distribution of the database RACs as a function of the number of features, and the number of standard deviations (SD) from the mean, for each cell. The number of features observed in each location constitutes the response of interest for this study and was used accordingly moving forward.

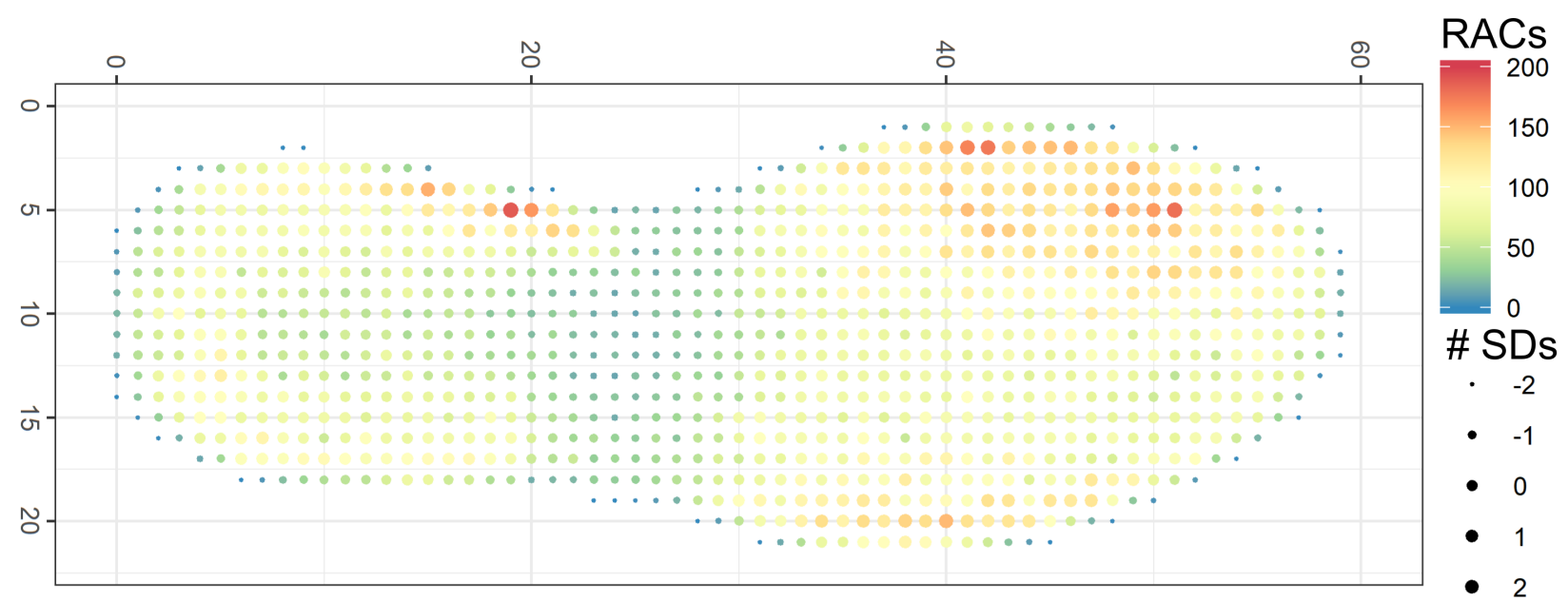

Figure 2: Number of RACs (color) and number of standard deviations from the mean (point size) for each cell location in the WVU database.

\section{Evaluation of RAC Distribution as a Function of Contact Area}

In order to assess the spatial distribution of RACs as a function of contact area within the standardized outsole, it was necessary to characterize the degree of tread area within 
each heatmap cell location. Subsequently, the average contact per bin (total contact/1,300) was computed. Additionally, since cells along the edge of the frame were incomplete, the percentage of each bin that fell within the study region was likewise characterized. Table 1 details the summary statistics for each of the predictors that were included in the models constructed for this phase. Across all locations, the mean contact was $10.3 \mathrm{~mm}^{2} \pm 3.30 \mathrm{~mm}^{2}$. Furthermore, the vast majority of cells on the heatmap were complete (approximately $83 \%$ had all pixels contained within the frame).

Table 1: Summary statistics of model predictors (average contact area and bin partiality).

\begin{tabular}{|c|c|c|}
\hline Metric & $\begin{array}{c}\text { Average Contact } \\
\left(\mathbf{m m}^{\mathbf{2}}\right)\end{array}$ & $\begin{array}{c}\text { Bin Partiality } \\
\mathbf{( \%} \text { on } \text { frame })\end{array}$ \\
\hline Mean & 10.3 & 92.8 \\
\hline SD & 3.30 & 20.9 \\
\hline Median & 11.4 & 100 \\
\hline IQR & $(8.62,12.4)$ & $(100,100)$ \\
\hline Minimum & 0.001 & 0.03 \\
\hline Maximum & 16.2 & 100 \\
\hline
\end{tabular}

\section{Model Fitting}

After checking for multicollinearity between the predictors using the variance-inflation factor (VIF < 10) [20, 24, 29], four models (non-spatial GLM, AP, Durbin, GWPR) were constructed to evaluate the relationship between tread contact and the number of observed RACs. For all spatial models, the neighborhood cut-off for the weight matrix was 800px $(\approx 34 \mathrm{~mm})$, beyond which values were not considered to impact each other. Table 2 details the estimated model coefficients for the three global models. All parameters provided evidence of significant impacts on RAC counts $\left(p<2.2 \times 10^{-16}\right)$. With the exception of the contact area spatial lag for the spatial Durbin model, all coefficients exhibited a positive impact on the number of features, wherein an increase in the predictor yields an inflation of the response. In other words, more contact within a region (by tread areas, bin totality, or both) results in more accidentals observed. This agreement between the various models offers 
strong evidence that this is the true effect underlying part of the data generating process and likewise fits with practical intuition about the development of RACs being dependent upon contact with the ground.

Table 2: Model coefficients for RAC counts as a function of contact area, with a bin percentage offset variable to adjust for partiality for three regression models of interest (non-spatial GLM, spatial auto-Poisson, and spatial Durbin). For all spatial models, a neighborhood cutoff value of 800px (approximately 34mm) was used.

\begin{tabular}{|c|c|c|c|c|c|}
\hline Model & $\begin{array}{c}\text { Intercept } \\
\left(\beta_{0}\right)\end{array}$ & $\begin{array}{c}\text { Contact } \\
\left(\beta_{1}\right)\end{array}$ & $\begin{array}{c}\text { Bin \% } \\
\left(\beta_{3}\right)\end{array}$ & $\begin{array}{c}\text { Spatial Lag } \\
\text { RACs } \\
(\rho)\end{array}$ & $\begin{array}{c}\text { Spatial Lag } \\
\text { Contact } \\
(\theta)\end{array}$ \\
\hline $\begin{array}{c}\text { Non-Spatial } \\
\text { GLM }\end{array}$ & 2.957 & $2.2 \mathrm{E}-04$ & 0.5944 & $\mathrm{NA}$ & $\mathrm{NA}$ \\
\hline $\begin{array}{c}\text { Spatial } \\
\text { AP }\end{array}$ & 2.540 & $1.2 \mathrm{E}-04$ & 0.9269 & 0.0133 & $\mathrm{NA}$ \\
\hline $\begin{array}{c}\text { Spatial } \\
\text { Durbin }\end{array}$ & 2.914 & $2.1 \mathrm{E}-04$ & 0.7314 & 0.0152 & $-1.0 \mathrm{E}-04$ \\
\hline
\end{tabular}

When considering the local modeling of RAC counts as a function of contact area (and adjusted for bin partiality), similar relationships were observed as those in the global models. Detailed in Table 3 are the summary statistics for the coefficient estimates obtained for 987 location-specific models built using GWPR. Again, all parameters exhibited a positive association with RAC frequency, with the exception of 30 cells along the edge of the toe that had a small negative relationship for contact area. It is likely that this relationship was observed because there is a large variability in both contact area (ranging from $0.124 \mathrm{~mm}^{2}$ to $14.5 \mathrm{~mm}^{2}$ ) and bin partiality (ranging from $1.59 \%$ to $100 \%$ ) at these locations. This high variability results in neighboring cells having dissimilar values (with RAC counts between 0 and 118), which appears to have ultimately imparted an inverse association.

\section{Evaluation of Model Residuals}

In an effort to evaluate the appropriateness of each model, as well as to determine whether there remains an unmodeled factor that impacts the spatial distribution of accidentals, analysis of the model residuals was conducted [15, 20, 24, 26]. More specifically, the obtained 
Table 3: Summary statistics for model coefficients for RAC counts as a function of contact area, with a bin percentage offset variable to adjust for partiality for the geographically weighted Poisson regression (GWPR). A neighborhood cutoff value of 800px (approximately 34mm) was used.

\begin{tabular}{|c|c|c|c|}
\hline Metric & $\begin{array}{c}\text { Intercept } \\
\left(\beta_{0}\right)\end{array}$ & $\begin{array}{c}\text { Contact } \\
\left(\beta_{1}\right)\end{array}$ & $\begin{array}{c}\text { Bin \% } \\
\left(\beta_{3}\right)\end{array}$ \\
\hline Mean & 3.064 & $2.1 \mathrm{E}-04$ & 0.5212 \\
\hline SD & 0.6937 & $1.0 \mathrm{E}-04$ & 0.0166 \\
\hline Median & 3.005 & $2.1 \mathrm{E}-04$ & 0.3746 \\
\hline Minimum & 2.145 & $-2.9 \mathrm{E}-05$ & 0.0074 \\
\hline Maximum & 4.777 & $3.7 \mathrm{E}-04$ & 2.250 \\
\hline
\end{tabular}

Pearson residuals were evaluated for the remaining presence of spatial autocorrelation using global and local Moran's I [15, 20, 24, 26, 30, 31]. Moran's I ranges from -1 for complete negative correlation to +1 for positive associations, with a lack of detectable spatial correlation resulting in a score near zero.

When compared to the non-spatial GLM, each of the spatial models shows an improvement in prediction, with more residuals near 0 and fewer at the extremes, as observed in Figure 3. Therefore, there appear to be spatial effects that impact RAC distributions, as expected based upon previous results. Furthermore, all models exhibited significant positive global spatial autocorrelation according to Moran's I analyses $\left(p<2.2 \times 10^{-16}\right)$. More specifically, the non-spatial GLM possessed the highest residual correlation with a global value of 0.5085 . Conversely, the spatial Durbin model yielded the lowest level of positive autocorrelation, with $I=0.1652$. The Auto-Poisson and GWPR regressions resulted in Moran's I scores of 0.2027 and 0.2364 , respectively.

In addition to global evaluation of spatial autocorrelation, local estimates of Moran's I were likewise computed for the residuals of each model, as illustrated in Figure 4. While the vast majority of significant correlations were positive (red dots), there were a limited number of cells that showed an inverse relationship with their neighbors (blue dots). Again, the non-spatial GLM produced the largest areas of localized associations, with approximately $39 \%$ of cells resulting in significant Moran's I values. Not surprisingly, these regions are in 

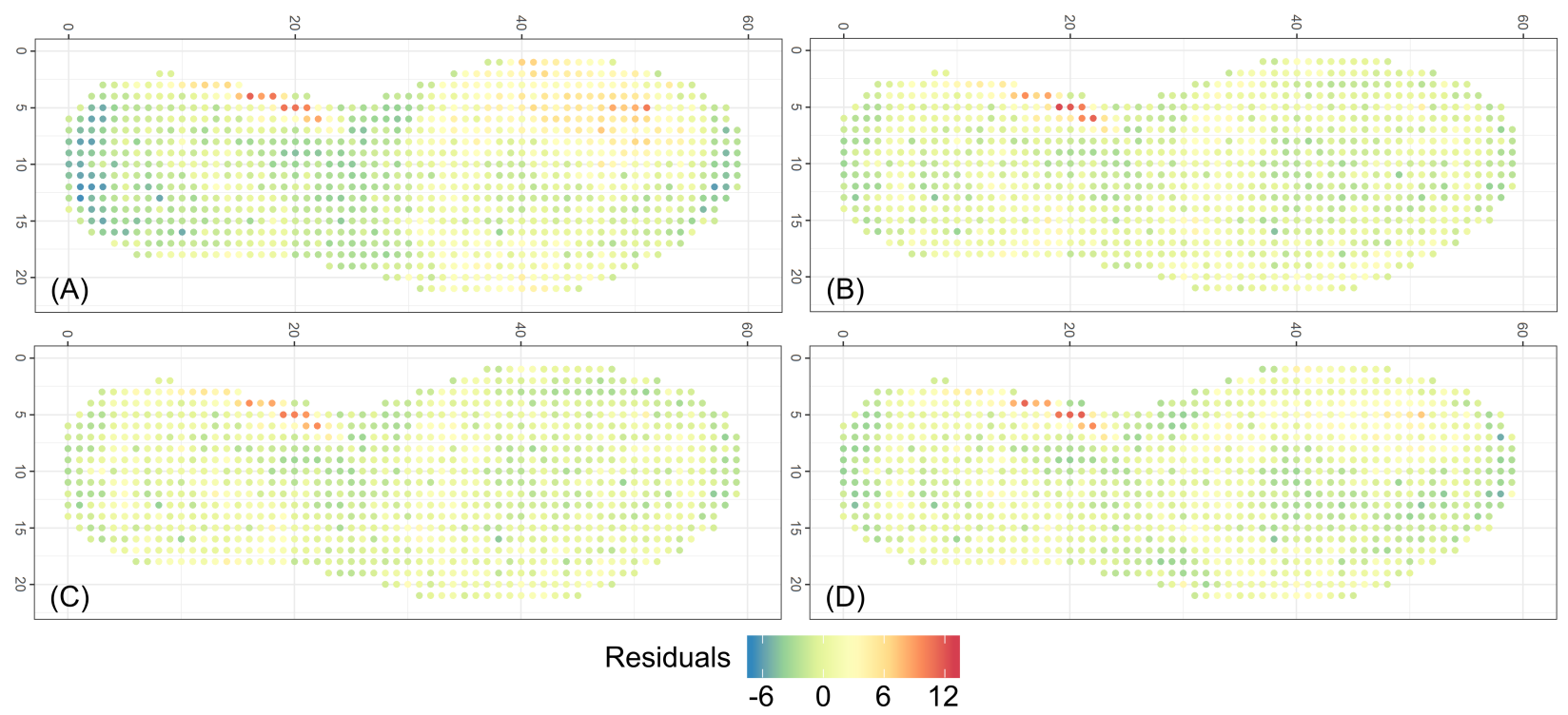

Figure 3: Pearson residuals (color) of RAC counts as a function of contact area (with a bin percentage offset) obtained from four different models and the full database of 1,300 shoes: (A) non-spatial Poisson GLM, (B) spatial auto-Poisson, (C) spatial Durbin, (D) geographically weighted Poisson regression. For all spatial models, a neighborhood cutoff value of 800px (approximately 34mm) was used.

alignment with the regions that were not well predicted by the Poisson simulations (as illustrated by the clustered red locations in Chapter 3, Figure 9), including the ball of the toe, the arch, and the edge of the heel. Furthermore, the observed residual analyses detailed in Chapter 3 indicated that an average of $36 \% \pm 1.6 \%$ cells likewise yielded significant correlations for the empirical versus 10,000 Poisson simulated heatmap realizations, which is again in alignment with these results. This observation is expected because the non-spatial GLM models empirical RAC counts uniformly as a function of contact across the entire study region, irrespective of location/neighbors. Similarly, the Poisson simulations describe the frequency of features assuming uniform point intensity and modified by tread contact. Therefore, because both analyses disregard the chance of spatial association between nearby locations number of accidentals and degree of contact, it was expected that their results would be comparable.

In terms of the spatial regression models, the same trend observed for the global Moran's I analysis was replicated for the local evaluations; the spatial Durbin model best reduced the spatial autocorrelation of the data, followed by the AP and then the GWPR regressions 
(yielding 13\%,16\%, and $22 \%$ significant cells, respectively). In order to compare the resulting proportions of significant residuals association between models, a McNemar's test was conducted. This test is a modification of a traditional chi-square test and allows for an evaluation of differences between dependent proportions via assessment of consistency across two conditions (two regressions built using the same dataset for this study) [29, 32, 33]. Given that the test is non-parametric, the only necessary assumptions are a dichotomous outcome variable where the two conditions are mutually exclusive [29, 32, 33]. For the purposes of this study, the binary outcome of interest was whether each of the 987 cells across the outsole exhibited significant or non-significant residual correlation according to local Moran's I evaluation. According to the McNemar's test results (Table 4), the spatial Durbin model offered significant improvements to RAC count predictions thereby yielding fewer cells with residual spatial associations, wherein the largest $p$-value computed was 0.0060 for the comparison versus the AP model (3\% difference in proportion $\approx 30$ cells).

Table 4: McNemar's test results comparing the proportion of cells exhibiting significant residual correlations for regression models estimated to predict RAC counts as a function of degree of tread contact.

\begin{tabular}{|c|c|c|}
\hline Model 1 & Model 2 & $\begin{array}{c}\text { McNemar's Test } \\
p \text {-value }\end{array}$ \\
\hline $\begin{array}{c}\text { Durbin } \\
(13 \%)\end{array}$ & $\begin{array}{c}\text { AP } \\
(16 \%)\end{array}$ & $5.958 \mathrm{E}-03$ \\
\hline $\begin{array}{c}\text { Durbin } \\
(13 \%)\end{array}$ & $\begin{array}{c}\text { GWPR } \\
(22 \%)\end{array}$ & $1.130 \mathrm{E}-10$ \\
\hline $\begin{array}{c}\text { Durbin } \\
(13 \%)\end{array}$ & $\begin{array}{c}\text { GLM } \\
(39 \%)\end{array}$ & $1.793 \mathrm{E}-40$ \\
\hline AP & GWPR & $5.791 \mathrm{E}-06$ \\
$(16 \%)$ & $(22 \%)$ & $8.915 \mathrm{E}-29$ \\
\hline $\begin{array}{c}\text { AP } \\
(16 \%)\end{array}$ & $\begin{array}{c}\text { GLM } \\
(39 \%)\end{array}$ & \\
\hline GWPR & GLM & \\
$(22 \%)$ & $(39 \%)$ & $2.943 \mathrm{E}-20$ \\
\hline
\end{tabular}

When considered collectively, the results of the evaluation of model residuals predicting RAC counts as a function of contact area support two major conclusions. First, the inclusion 
Residuals $24,26,34]$.
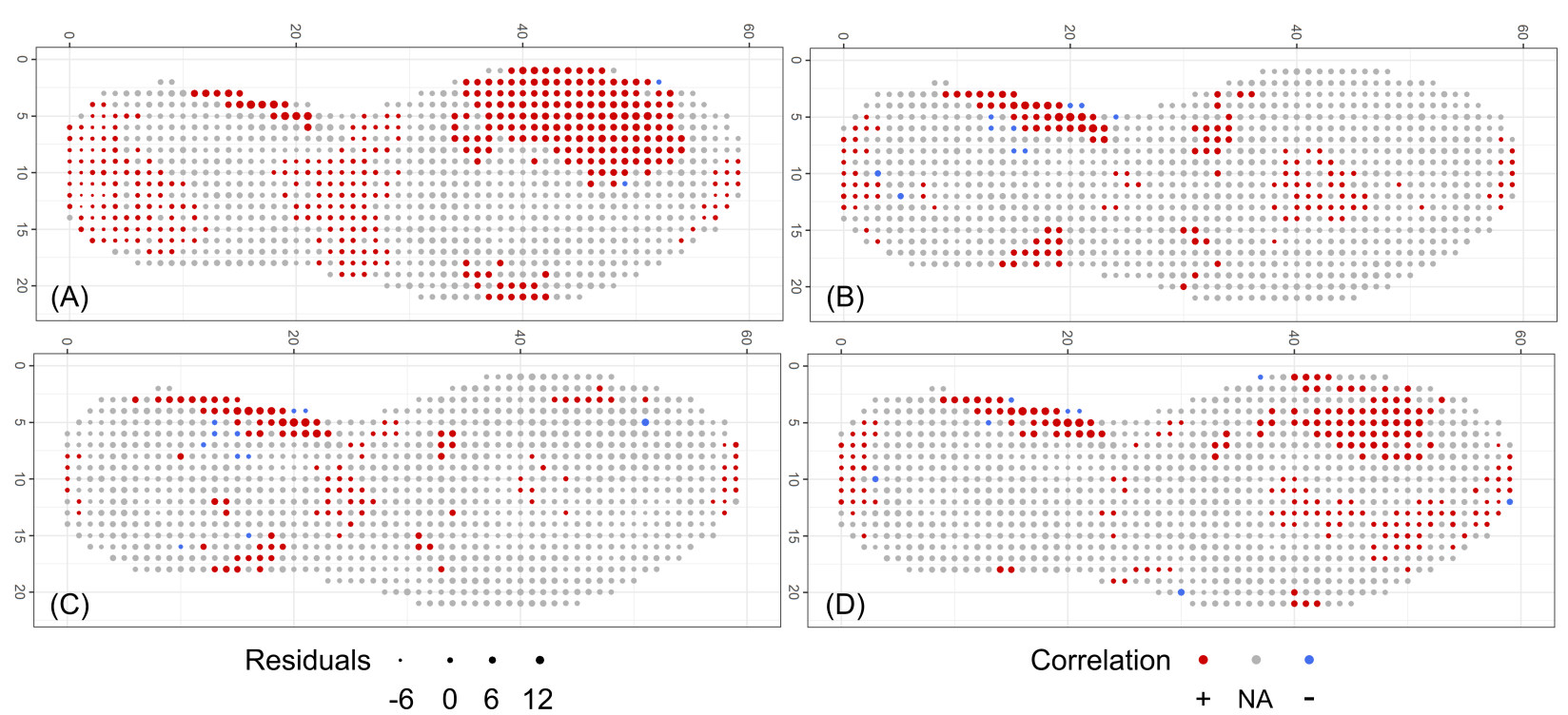

of sptial effects improves RAC count prediction. As supported by the significant decrease in spatial autocorrelation between the non-spatial GLM and all spatial models according to McNemar's test, and global Moran's I scores were 2-3× less, the incorporation of spatial lags improved the prediction of feature counts. Second, there is an unmodeled factor remaining that significantly impacts the distribution of accidentals, but only for some cells. If all relevant explanatory variables, including spatial effects, were accounted for, the resulting model residuals (under ideal circumstances) should not exhibit spatial correlation [15, 16,

Figure 4: Spatial correlation detected (red: positive, blue: negative, gray: none) and associated Pearson residuals (size) of RAC counts predicted as a function of contact area (with a bin percentage offset) using four different models: (A) non-spatial Poisson GLM, (B) spatial auto-Poisson, (C) spatial Durbin, (D) geographically weighted Poisson regression. For all spatial models, a neighborhood cutoff value of 800px (approximately $34 \mathrm{~mm}$ ) was used, representing the upper limit of the average lug size in the database.

\section{Model Assessment and Selection}

The performance of all four available models was compared using a variety of commonlyused model assessment statistics, including mean absolute deviation (MAD), mean squared error (MSE), and Akaike information criteria (AIC). Mean absolute deviation and mean squared error both represent the prediction error of the model and were computed according to Eq. $11[24,26,27,29]$, where $n$ is the number of locations (987 in this study), $Y_{i}$ is the 
number of RACs observed at location $i$, and $\hat{Y}_{i}$ is the predicted number of RACs expected at location $i$. Likewise, AIC is an estimator of model fit, but with a penalty for lack of parsimony $[29,35]$. The AIC for each polynomial was subsequently transformed into an estimate of the relative likelihood of the model (L(AIC)), according to the procedure detailed in Chapter 2 $[10,35]$. The computed L(AIC) represents the number of times the best-fitting model (with the lowest AIC) is more likely than the given model.

$$
\begin{aligned}
M A D & =\frac{\sum_{i=1}^{n}\left|Y_{i}-\hat{Y}_{i}\right|}{n} \\
M S E & =\frac{\sum_{i=1}^{n}\left(Y_{i}-\hat{Y}_{i}\right)^{2}}{n}
\end{aligned}
$$

Table 5 reports the resulting model assessment statistics for each of the four models estimating RAC counts as a function of tread contact area. According to all metrics, the spatial Durbin model most accurately predicts the distribution of feature frequencies. As previously described, the spatial Durbin model is the most robust regression technique owing to the fact that it accounts for spatial association between both the responses and the predictors $[15,19]$. Furthermore, even in the presence of spatially correlated errors, which is the scenario here as evidenced by the analysis of the model residuals, the spatial Durbin model produces unbiased estimates of the modeled coefficients $[15,19]$. However, this result should be taken with caution. More specifically, if there are additional factors that must be and can be accounted for, any predictions produced using the model may not be appropriate if such relevant explanatory variables are excluded. As such, given the significant spatial autocorrelation of residuals, contact area alone is not a sufficient predictor for the distribution of RACs on outsoles. While incorporation of contact area and spatial effects did improve model predictions, some cells still exhibited significant residual associations, and therefore another underlying factor should be considered. Visual observation of the residual clusters (Figures 3 \& 4) suggest that a factor such as wear, which is the interaction between contact as well gait/pressure across the outsole during usage, may be more informative in predicting accidental location and frequency. 
Table 5: Model assessment statistics describing the fit of four regressions of RAC counts as a function of contact area, with a bin percentage offset variable to adjust for partiality. The shaded cells reflect the best fit model according to MAD, MSE, AIC, and L(AIC). For all spatial models, a neighborhood cutoff value of 800px (approximately 34mm) was used, representing the upper limit of the average lug size in the database. Note: an $\mathrm{L}(\mathrm{AIC})$ value of $\operatorname{Inf}$ in the table represents $\mathrm{L}(\mathrm{AIC})>10^{305}$.

\begin{tabular}{|c|c|c|c|c|}
\hline Metric & $\begin{array}{c}\text { Non-Spatial } \\
\text { GLM }\end{array}$ & $\begin{array}{c}\text { Spatial } \\
\text { AP }\end{array}$ & $\begin{array}{c}\text { Spatial } \\
\text { Durbin }\end{array}$ & $\begin{array}{c}\text { Spatial } \\
\text { GWPR }\end{array}$ \\
\hline MAD & 18.59 & 11.24 & 10.92 & 13.12 \\
\hline MSE & 593.6 & 239.8 & 218.7 & 312.5 \\
\hline AIC & 13393 & 9119 & 8861 & 9938 \\
\hline L(AIC) & Inf & $1.05 \mathrm{E} 56$ & 1.00 & $7.37 \mathrm{E} 233$ \\
\hline
\end{tabular}

\section{Conclusions}

The objective of this research was to assess spatial RAC distributions as a function of the degree of tread contact in order to inform weight of evidence assessments for footwear source attribution comparisons and decisions. In pursuit of this objective, this research applied commonly-used spatial regression techniques in order to understand the "data-generating process" of feature locations and frequencies. The major benefits of using these techniques for this study are three-fold. First, implementation of various regression approaches, each considering a different type of spatial structure, allowed for the systematic determination of the optimal model to describe RAC distributions. The results indicated that accidentals are best modeled as a function of contact using a spatial Durbin model, which incorporates spatial associations in both the predictors (exogenous) and responses (endogenous) (Table 5). Second, an analysis of the estimated coefficients allows for an interpretation of the effect that the predictor(s) impart on the response of interest. More specifically for this study, the number of features observed within a given location increases as contact area increases (Tables 2). This observation reasonably fits with intuition that the greater the area of contact with the ground, the greater the chance of developing an accidental. Lastly, spatial regression modeling techniques allow for incorporation of spatial dependencies, wherein the strength and direction of correlation between predictors and/or responses and their neighbors can 
be assessed. Results indicated significant spatial effects for both RAC counts and contact between neighboring locations, as supported by the significant contributions of their spatial lag variables ( $\rho$ and $\theta$, respectively in Tables 2 ).

Specifically regarding the data-generating process of RACs with respect to contact, the results indicate that both endogenous and exogenous effects underpin the distribution of features on outsoles. This conclusion was reinforced by two statistical analyses - the McNemar's test for proportion of significant Moran's I cells and the L(AIC) for the model assessment statistics. However, there are some caveats that should be acknowledged along with this outcome. First, the inclusion of the exogenous associations (between predictors) only decreased the number of cells with persisting residual correlation by $3 \%$, as compared to endogenous effects (between responses) alone. While this change was statistically significant, the practical evaluation is more complex. Considering the estimated coefficients, inclusion of a spatial lag on contact area resulted in a negative coefficient, coupled with a slight inflation of the spatial lag on the responses, as detailed in Table 2. This outcome may indicate the presence of confounding, which fits with intuition regarding the underlying phenomenon. In other words, given that the degree of contact for neighboring cells is a significant predictor for RAC counts, it stands to reason that this association is likewise embedded within the endogenous factor (feature frequencies in nearby locations), which may explain the small change in proportions. Second, while the GWPR model did not optimally predict accidental distributions for this data at this fixed scale, this result does not suggest that heterogeneity does not exist in the data. In fact, consideration of heterogeneity via local modeling does significantly improve predictions over the non-spatial model $\left(p=2.9 \times 10^{-20}\right)$, but it does not adequately describe the stronger spatial correlations and thus under-performs as compared to both the AP and Durbin models (Table 4). Therefore, RAC distributions are better described using the best estimate shoe-wide (global modeling of spatial associations) rather than local estimates (local modeling of heterogeneity), at least for this fixed scale of 800px $(34 \mathrm{~mm})$.

While this study was successfully able to characterize several factors impacting the distribution of acquired features on outsoles, there were several limitations that must be noted. As previously discussed in Chapters 2 and 3, the tread contact maps were created using an 
automated image processing procedure wherein several morphological operators were applied to Handiprint images in order to segment contact pixels. While every effort was made to ensure high quality binary maps, the Handiprint images are inherently speckled given that they are created using black powder, and this fact precludes the production of perfect tread contact representations. A more supervised process, such as using a high resolution outsole scanner and/or manually annotating or modifying tread contact regions, may yield more accurate maps. However, this process would be both time- and cost- intensive and may not provide significant changes or improvements to the results obtained for this study to warrant the investment.

Furthermore, all contact maps were poly-warped to allow for inter-comparison of the entire WVU database of shoes. While this was a necessary step to increase the sample size available for analysis, this process likewise limits the utility of the results owing to the induced modification of tread appearance. To overcome this limitation, a database would need to be constructed that contains a large variety of outsoles each with numerous replicates to allow for examination within the same class characteristics. However, this undertaking would likely be difficult to implement in practicality given the cost, time, and resources associated with compiling such a large collection of shoes. Furthermore, it would be necessary to constantly expand the database as new outsole designs are produced, which could become prohibitive.

Despite the limitations outlined above, the results from this initial study are promising. As previously outlined, the results from Chapter 3 indicated that one or more important predictive factor(s) were missing to adequately describe the empirical RAC distributions observed for the WVU database. Moreover, these factors could have been theoretical (spatial heterogeneity or interaction between neighboring cells) and/or practical (another characteristic of the outsole or acquisition process) in nature. By keeping the outsole-related predictor constant (cell-specific contact area), this study sought to investigate the theoretical factors by implementing a variety of spatial regression techniques, wherein spatial associations between predictors and responses were incorporated. The results indicated that contact-area alone, irrespective of position, was able to adequately describe RAC frequencies for $61 \%$ of outsole locations, as supported by the data for the non-spatial GLM model. Furthermore, the locations exhibiting under-performance were clustered into a few major groups, with 
the ball of the toe, arch, medial heel, and the edge of the toe and heel containing significant residual associations (Figure 4). When allowing for spatial effects, by including spatial correlations between neighboring cells' feature counts and contact areas, $87 \%$ of the shoe is well predicted. Therefore, improved model predictions when utilizing spatial regressions indicate that spatial effects are important for characterizing RAC distributions. However, significant residual correlations persist in 22-13\% of outsole locations (Figure 4), suggesting that an informative practical factor remains unmodeled. Based upon the observed locations of significant residual associations, it was purported that wear could be one such factor. Consequently, a proof of concept study was conducted in order to investigate this hypothesis, which is detailed in Part II of this effort. 


\section{References}

[1] M. J. Cassidy, Footwear Identification, Lightning Powder Company, Inc., 1995.

[2] A. S. Fawcett, The role of the footmark examiner, Journal of the Forensic Science Society 10 (1970) 227-244.

[3] R. Stone, Footwear examination: Mathematical probabilities of theoretical individual characteristics, Journal of Forensic Identification 56 (2006) 577-599.

[4] T. W. Adair, J. Lemay, A. McDonald, R. Shaw, R. Tewes, The Mount Bierstadt study: An experiment in unique damage formation in footwear, Journal of Forensic Identification 57(2) (2007) 199-205.

[5] C. Hamburg, R. Banks, Evaluation of the random nature of acquired marks on footwear outsoles, Impression and Pattern Evidence Symposium, 2010.

[6] H. Wilson, Comparison of the individual characteristics in the outsoles of thirty-nine pairs of Adidas Supernova Classic shoes, Journal of Forensic Identification 62(3) (2012) 194-203.

[7] Y. Yekutieli, Y. Shor, S. Wiesner, T. Tsach, Expert Assisting Computerized System for Evaluating the Degree of Certainty in 2D Shoeprints, Technical Report, National Institute of Justice, 2012.

[8] N. Petraco, C. Gambino, T. Kubic, D. Olivio, N. Petraco, Statistical discrimination of footwear: A method for the comparison of accidentals on shoe outsoles inspired by facial recognition techniques, Journal of Forensic Sciences 55 (2010) 34-41.

[9] M. Marvin, Oral presentation: A look at close non-matching footwear examinations, in: International Association for Identification (IAI) Centennial Conference.

[10] N. Richetelli, W. Bodizak, J. Speir, Empirically observed and predicted estimates of chance association: Estimating the chance association of randomly acquired characteristics in footwear comparisons, Forensic Science International 302 (2019) 109833. 
[11] W. J. Bodziak, Forensic Footwear Evidence, Taylor \& Francis Inc, 2016.

[12] N. Richetelli, M. Nobel, W. J. Bodziak, J. A. Speir, Quantitative assessment of similarity between randomly acquired characteristics on high quality exemplars and crime scene impressions via analysis of feature size and shape, Forensic Science International 270 (2017) 211-222.

[13] J. LeSage, The Theory and Practice of Spatial Econometrics, University of Toledo, 1999.

[14] J. LeSage, An introduction to spatial econometrics, Revue d'économie industrielle 123 (2008) 19-44.

[15] Handbook of Spatial Analysis: Theory and Application with R, The National Institute of Statistics and Economic Studies (INSEE), 2018.

[16] W. Koenig, Spatial autocorrelation and local disappearances in wintering north american birds, Ecology 82(9) (2001) 2636-2644.

[17] J. VerHoef, E. Peterson, M. Hooten, E. Hanks, M. Fortin, Spatial autoregressive models for statistical ecological data, Ecological Monographs 88(1) (2018) 33-59.

[18] J. Lichstein, T. Simons, S. Shriner, K. Franzreb, Spatial autocorrelation and autoregressive models in ecology, Ecological Monographs 72(3) (2002) 445-463.

[19] J. Elhorst, Applied spatial econometrics: Raising the bar, Spatial Economic Analysis 5(1) (2010) 9-28.

[20] P. Simões, I. Natário, Spatial econometric approaches for count data: An overview and new directions, International Journal of Social, Behavioral, Eduational, Economic, Business and Industrial Engineering 10(1) (2016) 348-357.

[21] S. Glaser, A review of spatial econometric models for count data, Hohenheim Discussion Papers in Business, Economic and Social Sciences 19 (2017).

[22] G. Jacquez, Handbook of Regional Science, Springer, pp. 1311-1333.

[23] R. Haining, Handbook of Regional Science, Springer, pp. 1277-1294. 
[24] Z. Ma, B. Zuckerberg, W. Porter, L. Zhang, Spatial Poisson models for examining the influence of climate and land cover pattern on bird species richness, Forest Science 58(1) (2012) 61-74.

[25] R. Florax, H. Folmer, S. Rey, Specification searches in spatial econometrics: the revelance of Hendry's methodology, Regional Science and Urban Economics 33 (2003) $557-579$.

[26] Z. Li, W. Wang, P. Liu, J. Bigham, D. Ragland, Using geographically weighted Poisson regression for county-level crash modeling in california, Safety Science 58 (2013) 89-97.

[27] L. Zhang, Z. Ma, L. Guo, An evaluation of spatial autocorrelation and heterogeneity in the residuals of six regression models, Forest Science 55(6) (2009) 533-548.

[28] J. Speir, N. Richetelli, M. Fagert, M. Hite, W. J. Bodziak, Technical note: Quantifying randomly acquired characteristics on outsoles in terms of shape and position, Forensic Science International 266 (2016) 399-411.

[29] R. J. Freund, W. J. Wilson, D. L. Mohr, Statistical Methods, Academic Press, 3rd edition, 2010.

[30] O. Schabenberger, C. Gotway, Statistical Methods for Spatial Data Analysis, Chapman \& Hall/CRC, 2005.

[31] L. Anselin, Local indicators of spatial association - LISA, Geographical Analysis 27(2) (1995) 93-115.

[32] W. Conover, Practical Nonparametric Statistics, John Wiley \& Sons, Inc., 3rd edition, 1999.

[33] N. Salkind, Encyclopedia of Research Design, SAGE Publications, Inc., 2010.

[34] M. Cardillo, L. Brohman, S. Greenhill, Links between language diversity and species richness can be confounded by spatial autocorrelation, Proceedings of the Royal Society B: Biological Sciences 282 (2015) 20142986. 
[35] E. Wagenmakers, S. Farrell, AIC model selection using Akaike weights, Psychonomic Bulletin \& Review 11 (2004) 192-196. 
5. Spatial Modeling of RACs as a Function of Outsole Factors: Part II 


\title{
Spatial Regression Modeling of Randomly Acquired Characteristics on Outsoles with Implications Regarding Weight of Evidence Determinations: Part II
}

\begin{abstract}
Keywords: Forensic Footwear, Randomly Acquired Characteristics, Weight of Evidence, Spatial Regression Modeling, Spatial Autocorrelation
\end{abstract}


their prediction, additional work was conducted using spatial regression modeling.

Part I of this research effort (Chapter 4 of this document) specifically focused on determining whether spatial effects were contributing to the frequencies of features observed across a standardized heatmap outsole. In other words, using the same database of RACs as well as the tread contact information, four regression approaches were applied to the data in order to determine the degree to which associations between neighboring responses (RAC counts) and predictors (average contact area) impacted the empirical accidental distributions. The results indicated that both exogenous and endogenous spatial effects significantly influenced RAC distributions, as evidenced by significant contributions of the spatial lags between both the feature frequencies $(\rho)$ and underlying contact $(\theta)$ (Table 2, Chapter 4). Moreover, there was a $67 \%$ reduction in significant residual spatial correlation between the non-spatial model and the optimal model (spatial Durbin), indicating better prediction performance overall.

Following analysis of RAC distributions as a function of tread contact area using spatial regression modeling to account for spatial effects in the response as a function of the predictors, attention was then focused on whether consideration of an additional outsole factor could improve predictions. Based upon the regions exhibiting low prediction performance (large residual deviations from 0 and significant residual spatial correlations), it was theorized that contact-localized degree of wear may be one such explanatory factor. Since this variable represents an intersection of both outsole design (tread contact) and shoe use (gait/pressure on specific outsole regions), it was hypothesized that wear could improve model predictions. Therefore, the main objective of this work (Part II) was to conduct a proof of concept study to compare regression performance using contact area and contact-localized wear as explanatory factors for RAC distributions.

\section{Materials and Methods}

\section{Database and Pre-Processing}

In order to assess the impact of wear on spatial modeling, a subset of the WVU footwear database was selected for analysis. In order to maximize the number of RACs, all 1,300 shoes were sorted based upon the number of RACs they exhibited, and the top 10\% (130 
shoes) were selected for this phase, yielding 27,933 accidentals available for assessment (39\% of the RACs).

\section{Modeling of RAC Distribution as a Function of Contact Area}

The contact models (described in detail in Chapter 4) were re-estimated on the subset data, according to Eqs. 1-4. The goals of this reanalysis were two fold. First, the results from this analysis allow for a comparison of the full database to the proof of concept data to ensure that the subset is representative of the observations and conclusions reached when considering all 1,300 shoes. In addition, this allowed for a direct evaluation of contact versus wear as predictors for the distribution of RACs on outsoles, with implications including model performance and residual spatial autocorrelation.

$$
\begin{aligned}
& \log (R)=\beta_{0}+\beta_{1} C+\beta_{2} \log (B) \\
& \log (R)=\beta_{0}+\beta_{1} C+\beta_{2} \log (B)+\rho W_{R} \\
& \log (R)=\beta_{0}+\beta_{1} C+\beta_{2} \log (B)+\rho W_{R}+\theta W C \\
& \log (R)=\beta_{0}\left(u_{i}, v_{i}\right)+\beta_{1} C\left(u_{i}, v_{i}\right)+\beta_{2} \log \left(B\left(u_{i}, v_{i}\right)\right)
\end{aligned}
$$

For this proof of concept investigation of the distribution of 27,933 RACs, three neighborhood cut-off values (400px/17mm, 800px/34mm, and $1200 \mathrm{px} / 51 \mathrm{~mm})$ were used in the weight matrix of all spatial models to additionally evaluate whether there were scale dependent effects. More specifically, $17 \mathrm{~mm}$ was selected because this value represents the average lug diameter in the database. Likewise, $34 \mathrm{~mm}$ was included because this was determined to be the upper limit (mean $+2 \mathrm{SD}$ ) of lug diameter. Lastly, 51mm was used to allow for exploration of RAC distributions that fall on different tread elements.

\section{Localization of Outsole Wear}

As previously described, the WVU database contains a variety of shoes, with different class and subclass characteristics. Likewise, these shoes exhibit considerable variation in outsole condition, specifically regarding the locations and degree of wear. While all shoes were previously assigned a global level of wear (light, moderate, or high) [3], this characteristic 
was never evaluated locally. In order to describe the degree of wear at each position across the outsole, wear maps were created using the criteria described in Table 1. This process required an analyst to examine each shoe's outsole and its associated Handiprint exemplar (both digitized at 600ppi) in Adobe ${ }^{\circledR}$ Photoshop ${ }^{\circledR}$ Elements 10. A semi-transparent mask layer was added over the Handiprint image in order to annotate the location and degree of wear across the entire frame of the shoe. More specifically, the annotation layer originated as white (grayscale value of 255) indicating a moderate degree of wear, since it was expected that this would be the most prevalent category based upon the global classifications [3]. Each shoe's images (outsole and Handiprint simultaneously) were then methodically evaluated for the presence of light and high wear. When identified, the free-form polygon tool was used to outline the region of interest and the paint-bucket tool was used to fill the region with a specific grayscale value associated with the wear category (values of 50 and 175 for high and light, respectively). Finally, the moderate wear areas were reviewed to ensure adherence to necessary criteria, any required adjustments were made, and the wear map layer was saved as its own image. Figure 1 displays outsoles, Handiprints, and resulting wear maps for three shoes in the subset database.

Following creation of wear maps for each of the 130 shoes, each image was polywarped in the same manner as the binary contact maps (described in Chapter 3). Wear areas were then localized to contact pixels and segmented into corresponding heatmap bins based upon coordinates, thus resulting in data representing the intersection between contact and degree of wear on the standardized heatmap frame. Subsequently, the amount of contact-localized wear within each bin and of each degree classification was determined for each shoe and then summed across all shoes to obtain the total wear at each location. Lastly, each summation was divided by the total number of shoes (130) to represent the average contact-localized wear for light, moderate, and high degrees. For the purposes of this work, any discussion of "wear" indicates the average amount of contact area intersected by each wear category in each cell. For example, the light wear predictor represents the average number of contact pixels at that position that also exhibited light wear. 
Table 1: Criteria for assigning localized degrees of wear. Note: examples of regions classified as each level are provided in Figure A.1 of the Appendix.



Figure 1: Wear map examples for three shoes in the database, including the outsole (left), Handiprint (center), and resulting wear map (right) images. Note that the wear maps display the original images prior to poly-warping and contact localization. 


\section{Modeling of RAC Distribution as a Function of Wear}

For each model, the number of RACs at each heatmap location was the observed response variable $(R)$, while the average contact-localized wear for light, moderate, and high degrees ( $L_{C}, M_{C}$, and $H_{C}$, respectively) were used as predictors. In addition, the bin partiality (percentage of the bin within the heatmap frame) was again included as an offset variable $(B)$. As an example, suppose a full cell $(\log (B)=\log (1.0))$ contains 24 total RACs $(R$ $=24$ ) originating from the 130 shoes in the proof of concept database. In addition, there are 719,286 contact pixels across all outsoles within the given cell. Each contact pixel is then assigned a wear category via intersection with the wear maps, resulting in a total of 105,213 light, 549,513 moderate, and 64,560 high contact-localized wear pixels. Finally, the average degree of contact-localized wear for each category was computed by dividing the total number of pixels by 130 shoes, thereby yielding the set of predictors $\left(L_{C}=809.331\right.$, $\left.M_{C}=4,227.02, H_{C}=496.615\right)$ for the models described in Eqs. 5-8. Akin to the approach taken for assessment of RACs as a function of contact area, four models were constructed to evaluate the distribution of accidentals using degree of wear and incorporating spatial effects

(Eqs. 5-8). In addition, contact-localized wear analyses were likewise conducted using three neighborhood sizes to allow for a direct comparison of results.

$$
\begin{aligned}
& \log (R)=\beta_{0}+\beta_{1} L_{C}+\beta_{2} M_{C}+\beta_{3} H_{C}+\beta_{4} \log (B) \\
& \log (R)=\beta_{0}+\beta_{1} L_{C}+\beta_{2} M_{C}+\beta_{3} H_{C}+\beta_{4} \log (B)+\rho W_{R} \\
& \log (R)=\beta_{0}+\beta_{1} L_{C}+\beta_{2} M_{C}+\beta_{3} H_{C}+\beta_{4} \log (B)+\rho W_{R}+\theta_{1} W L_{C}+\theta_{2} W M_{C}+\theta_{3} W H_{C} \\
& \log (R)=\beta_{0}\left(u_{i}, v_{i}\right)+\beta_{1} L_{C}\left(u_{i}, v_{i}\right)+\beta_{2} M_{C}\left(u_{i}, v_{i}\right)+\beta_{3} H_{C}\left(u_{i}, v_{i}\right)+\beta_{4} \log \left(B\left(u_{i}, v_{i}\right)\right)
\end{aligned}
$$

\section{Results and Discussion}

\section{Database and Pre-Processing}

After subsetting the RAC database to include features contained on 130 shoes and discretized to 987 heatmap cells, there was an average of $28 \pm 16$ accidentals in each location, with a maximum of 76 . Interestingly, the observed mean and standard deviation are approximately $40 \%$ of those obtained for the full database which aligns with the proportion of 
all features comprising the subset data (39\%). Figure 2 illustrates the spatial distribution of the 27,933 RACs as a function of the number of features and the number of standard deviations (SD) from the mean. The number of features observed in each location constitutes the response of interest for this proof of concept study.

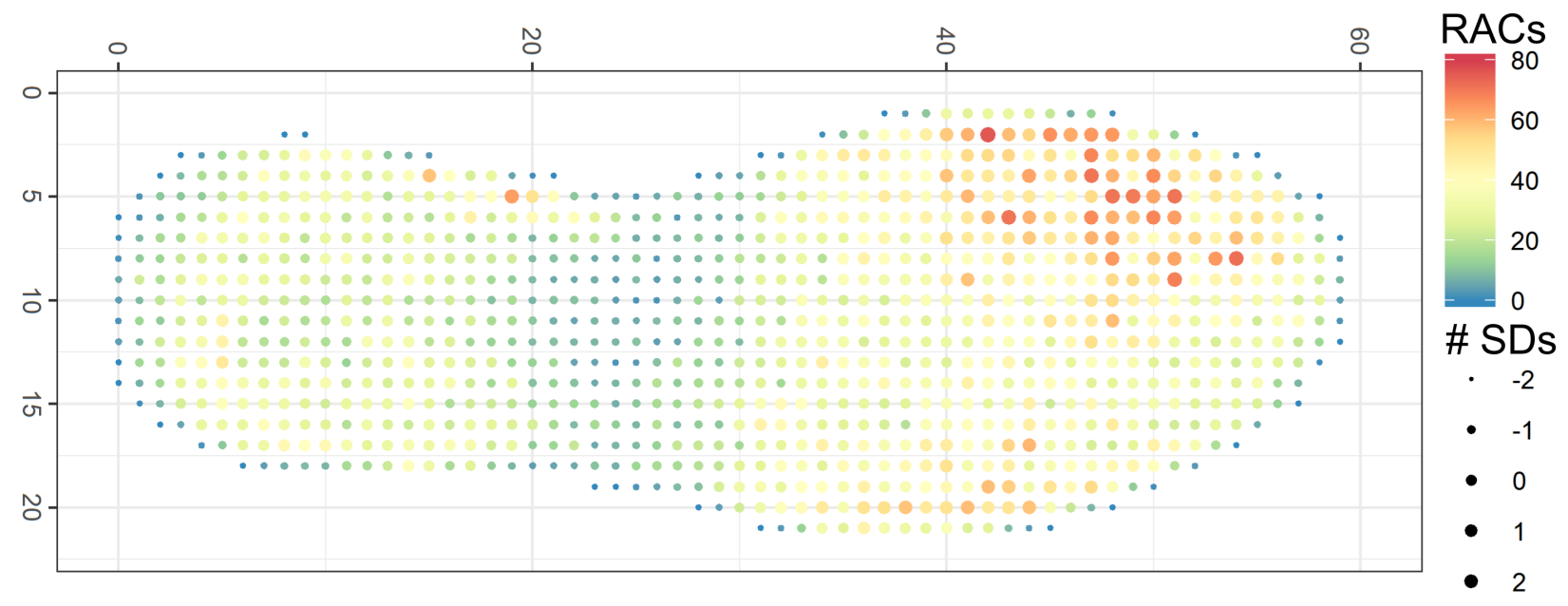

Figure 2: Number of RACs (color) and number of standard deviations from the mean (point size) for each cell location in the subset database. Note that the spatial distribution of RACs in the subset data is visually consistent with that for the full database, illustrated in Figure 2 of Part I.

\section{Modeling of RAC Distribution as a Function of Contact Area}

Following selection of the subset data, it was prudent to ensure that the observations and conclusions obtained for the prediction of RACs as a function of contact area in the full database persisted in the subset. To do so, the subset data was modeled in the same manner as previously described (Eq. 1-4) using a neighborhood cut-off value of 800px (34mm), thus allowing for direct comparison of results. Table 2 details the estimated coefficients for each of the four spatial regression models built using the full versus the subset data. For both datasets, all model parameters significantly impacted RAC counts $\left(p<2.2 \times 10^{-16}\right)$. Furthermore, all parameters exhibited a positive association with the number of accidentals, with the exception of the contact spatial lag in the Durbin model $(\theta)$.

When considering the contribution of the bin partiality offset $\left(\beta_{2}\right)$, the subset data exhibits higher coefficient values for each model than those obtained for the full database (with 
differences ranging from 0.1941 to 0.3489 as detailed in Table 2). It is important to note that this offset variable only impacts the model for edge bins (15\% of all cells), and for any locations contained fully within the heatmap frame this variable simplifies to $0(\log (1.0))$. Therefore, this coefficient describes some of the hardest to predict bins given the variability in number of RACs relative to inherently lower contact. Moreover, since the subset database only represents $39 \%$ of all features in the database there are likewise fewer accidentals in the edges, thereby requiring increased contribution to adequately fit the model. As such, this result is likely introduced based solely on the fact that fewer features are available in the subset database.

Another observable difference in the coefficients between the full and subset data is the magnitude of the auto-response variable $(\rho)$ for the AP and Durbin models (Table 2). More specifically, the spatial lag on RAC counts increased 2.3-2.5 times in the subset over the full model, as displayed in Table 2. This observation is believed to be an artifact based upon the composition of the subset database. Namely, the 130 shoes selected were those that contained the most RACs. Thus, these are the outsoles that exhibit the most consistency in RAC counts versus contact area, as compared to shoes with few accidentals. In other words, including all shoes in the full database model somewhat quenches the association in counts between nearby locations because although a great deal of additional tread area is being added, comparatively few features are being incorporated leading to increased variability. Therefore, given the high number of RACs relative to mean contact in the subset, a stronger spatial relationship is observed between neighboring cells' responses.

Analysis of model residuals was again conducted using global and local Moran's I to determine whether similar residual spatial associations were detected between the full and subset data. Pearson residual plots for both datasets are available in the Appendix (Figures A.2 \& A.3). In both cases, each of the spatial models shows an improvement in prediction over the non-spatial GLM as exhibited by fewer residual values near the extremes of the scale, thus providing evidence that spatial effects impact RAC distributions. In addition, all models exhibited significant positive global spatial autocorrelation, with Global Moran's I values ranging from 0.1089-0.5552 and from 0.1652-0.5085 for subset and full databases, respectively (Table 3). Investigation of local spatial autocorrelation likewise revealed that 
Table 2: Model coefficients for full and subset data predicting RAC counts as a function of contact area, with a bin percentage offset variable to adjust for bin partiality. For all spatial models, a neighborhood cutoff value of $800 \mathrm{px}$ (approximately 34mm) was used. Note that the results presented for the GWPR model are the mean coefficients for each predictor across all 987 cell-specific models.

\begin{tabular}{|c|c|c|c|c|c|c|c|c|}
\hline & \multicolumn{4}{|c|}{ Full Data } & \multicolumn{3}{c|}{ Subset Data } \\
\cline { 2 - 9 } & $\begin{array}{c}\text { Non-Spatial } \\
\text { GLM }\end{array}$ & $\begin{array}{c}\text { Spatial } \\
\text { AP }\end{array}$ & $\begin{array}{c}\text { Spatial } \\
\text { Durbin }\end{array}$ & $\begin{array}{c}\text { Spatial } \\
\text { GWPR }\end{array}$ & $\begin{array}{c}\text { Non-Spatial } \\
\text { GLM }\end{array}$ & $\begin{array}{c}\text { Spatial } \\
\text { AP }\end{array}$ & $\begin{array}{c}\text { Spatial } \\
\text { Durbin }\end{array}$ & $\begin{array}{c}\text { Spatial } \\
\text { GWPR }\end{array}$ \\
\hline $\begin{array}{c}\text { Intercept } \\
\left(\beta_{0}\right)\end{array}$ & 2.957 & 2.540 & 2.914 & 3.064 & 2.376 & 1.752 & 1.916 & 2.480 \\
\hline $\begin{array}{c}\text { Contact } \\
\left(\beta_{1}\right)\end{array}$ & 0.0002 & 0.0001 & 0.0002 & 0.0002 & 0.0002 & 0.0001 & 0.0001 & 0.0002 \\
\hline $\begin{array}{c}\text { Log(Bin) } \\
\left(\beta_{2}\right)\end{array}$ & 0.5944 & 0.9269 & 0.7314 & 0.6032 & 0.9433 & 1.121 & 0.9870 & 0.9429 \\
\hline $\begin{array}{c}\text { Spatial Lag } \\
\text { RACs } \\
(\rho)\end{array}$ & NA & 0.0133 & 0.0152 & NA & NA & 0.0340 & 0.0356 & NA \\
\hline $\begin{array}{c}\text { Spatial Lag } \\
\text { Contact } \\
(\theta)\end{array}$ & NA & NA & -0.0001 & NA & NA & NA & -0.0001 & NA \\
\hline
\end{tabular}

the subset models generally yielded fewer cells with significant residual association (11$17 \%$ versus 13-22\%), with the exeption of the non-spatial GLM (Table 3). Furthermore, the general regions containing these significant residual correlations remain consistent, as illustrated in Figure 3. For example, all models exhibit over-predicted RAC counts along the edge of the heel resulting in positive spatial autcorrelation in this region. Conversely, the medial edge of the upper heel/lower arch results in positive association between residuals yielded from under-prediction of feature frequencies. Some models also contain localized spatial correlation in the ball of the toe and the arch area between the full and subset data. The examination of model residuals indicate that irrespective of the input data, spatial effects do exist and consequently impact RAC distributions and that there still remains an unmodeled factor that is important for adequately predicting the locations and number of features across outsoles.

Lastly, the performance of all four available models was compared within each dataset using MAD, MSE, AIC, and L(AIC) as previously described. Table 4 details the computed model assessment metrics for the estimated models for the full and subset data. According 
Table 3: Moran's I results for full and subset data describing the global Moran's I value and the number of significant bins for local assessment. For all spatial models, a neighborhood cutoff value of 800px (approximately $34 \mathrm{~mm}$ ) was used.

\begin{tabular}{|c|c|c|c|c|c|c|c|c|}
\hline & \multicolumn{4}{|c|}{ Full Data } & \multicolumn{4}{c|}{ Subset Data } \\
\cline { 2 - 9 } & $\begin{array}{c}\text { Non-Spatial } \\
\text { GLM }\end{array}$ & $\begin{array}{c}\text { Spatial } \\
\text { AP }\end{array}$ & $\begin{array}{c}\text { Spatial } \\
\text { Durbin }\end{array}$ & $\begin{array}{c}\text { Spatial } \\
\text { GWPR }\end{array}$ & $\begin{array}{c}\text { Non-Spatial } \\
\text { GLM }\end{array}$ & $\begin{array}{c}\text { Spatial } \\
\text { AP }\end{array}$ & $\begin{array}{c}\text { Spatial } \\
\text { Durbin }\end{array}$ & $\begin{array}{c}\text { Spatial } \\
\text { GWPR }\end{array}$ \\
\hline $\begin{array}{c}\text { Global } \\
\text { Moran's I }\end{array}$ & 0.5085 & 0.2027 & 0.1652 & 0.2364 & 0.5552 & 0.1201 & 0.1089 & 0.1662 \\
\hline $\begin{array}{c}\text { Local } \\
\text { Moran's I }\end{array}$ & $39 \%$ & $16 \%$ & $13 \%$ & $22 \%$ & $40 \%$ & $12 \%$ & $11 \%$ & $17 \%$ \\
\hline
\end{tabular}

to all statistics, the spatial Durbin model most accurately predicts the distribution of RACs in both cases, followed by AP, GWPR, and finally the non-spatial GLM.

Table 4: Model assessment statistics for full and subset data describing the fit of four regressions of RAC counts as a function of contact area, with a bin percentage offset variable to adjust for partiality. The shaded cells reflect the best fit model according to MAD, MSE, AIC, and L(AIC). For all spatial models, a neighborhood cutoff value of 800px was used (approximately $34 \mathrm{~mm}$ ).

\begin{tabular}{|c|c|c|c|c|c|c|c|c|}
\hline \multirow{2}{*}{ Metric } & \multicolumn{4}{|c|}{ Full Data } & \multicolumn{4}{c|}{ Subset Data } \\
\cline { 2 - 9 } & $\begin{array}{c}\text { Non-Spatial } \\
\text { GLM }\end{array}$ & $\begin{array}{c}\text { Spatial } \\
\text { AP }\end{array}$ & $\begin{array}{c}\text { Spatial } \\
\text { Durbin }\end{array}$ & $\begin{array}{c}\text { Spatial } \\
\text { GWPR }\end{array}$ & $\begin{array}{c}\text { Non-Spatial } \\
\text { GLM }\end{array}$ & $\begin{array}{c}\text { Spatial } \\
\text { AP }\end{array}$ & $\begin{array}{c}\text { Spatial } \\
\text { Durbin }\end{array}$ & $\begin{array}{c}\text { Spatial } \\
\text { GWPR }\end{array}$ \\
\hline MAD & 18.59 & 11.24 & 10.92 & 13.12 & 9.388 & 5.716 & 5.656 & 6.918 \\
\hline MSE & 593.6 & 239.8 & 218.7 & 312.5 & 153.7 & 56.79 & 55.99 & 68.52 \\
\hline AIC & 13393 & 9119 & 8861 & 9938 & 9883 & 6903 & 6866 & 7162 \\
\hline L(AIC) & Inf & $1.1 \mathrm{E} 56$ & 1.0 & $7.4 \mathrm{E} 233$ & Inf & $1.1 \mathrm{E} 08$ & 1.0 & $1.9 \mathrm{E} 64$ \\
\hline
\end{tabular}

In conclusion, the subset data does show some variation in specific results when compared to the full database, as expected owing to underlying variation in contact and associated RACs. However, the results are in alignment when considering global observations and conclusions. Namely, all model coefficients yield significant impacts on the prediction of RAC counts and the directionality of these effects is consistent between datasets. Similarly, significant positive global autocorrelation persists throughout all model residuals in both cases, suggesting that some cells would benefit from a currently unmodeled predictor. Likewise, local residual associations exist in similar regions when comparing the same model between 1,300 and 130 shoes. The spatial Durbin model is the most optimal regression to 


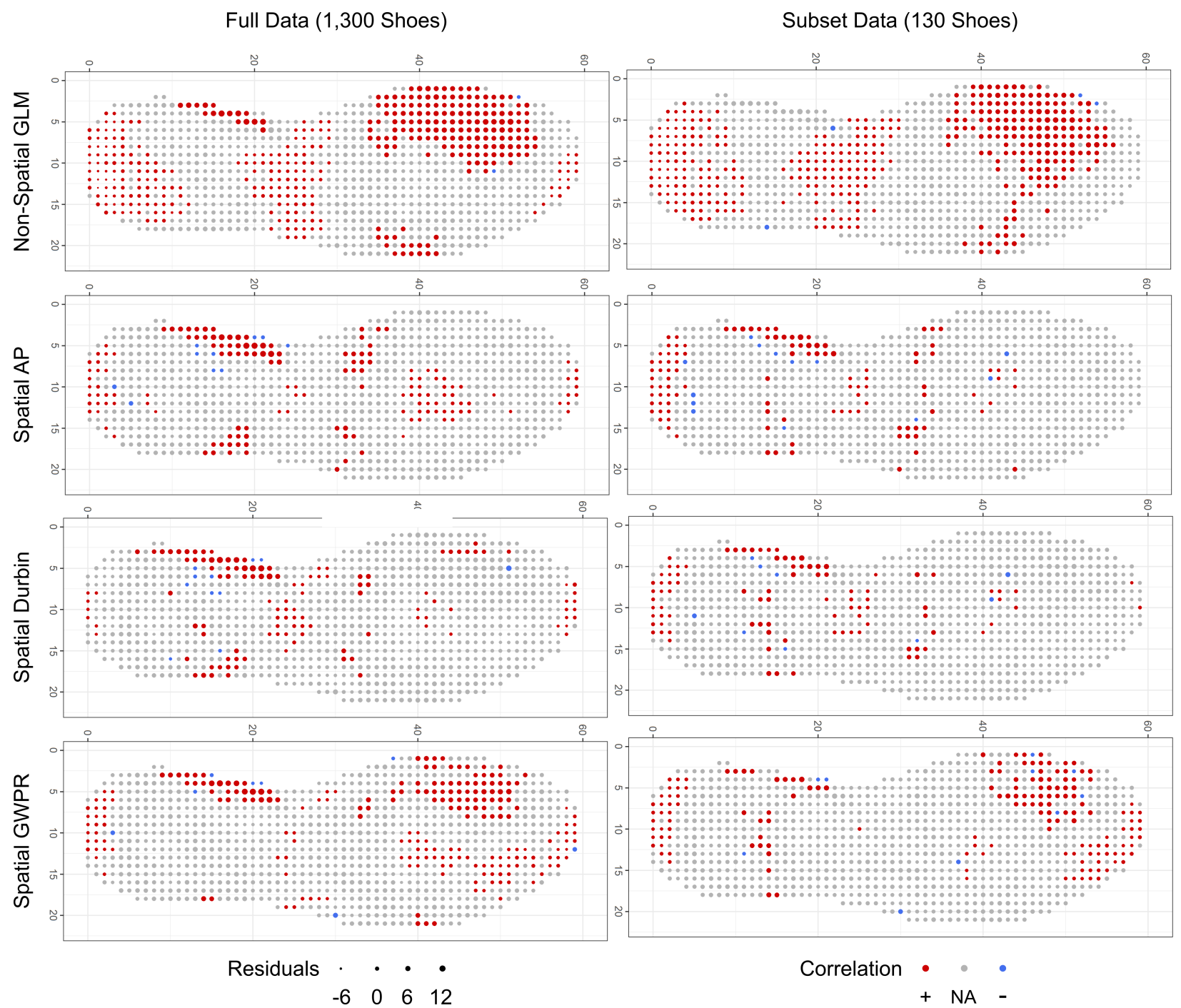

Figure 3: Spatial correlation detected (red: positive, blue: negative, gray: none) and associated Pearson residuals (size) of RAC counts predicted as a function of contact area (with a bin percentage offset) for full and subset data (1,300 and 130 shoes, respectively). For all spatial models, a neighborhood cutoff value of $800 \mathrm{px}$ (approximately $34 \mathrm{~mm}$ ) was used.

describe the data, indicating that association exists in RAC counts, likely as a function of the correlation between neighboring contact conditions. Ultimately, the collective results obtained based upon consideration of estimated model coefficients, residuals, and assessment statistics indicate that the subset data sufficiently represents the overall trends and resulting conclusions. 


\section{Localization of Outsole Wear}

Contact-localized wear areas were characterized for all 130 shoes in the subset database in order to evaluate the spatial distribution of RACs as a function of degree of wear. More specifically, the average amount of wear at each location was computed for low, moderate, and high degrees by dividing the total number of contact pixels containing each level of wear by the number of shoes (130). Figure 4 displays the spatial distribution of each degree of wear as well as a fraction of the total contact area within each cell. Across all locations, there was an average contact-localized wear area of $1.48 \mathrm{~mm}^{2} \pm 0.846 \mathrm{~mm}^{2}$ for light, $7.76 \mathrm{~mm}^{2} \pm 2.95 \mathrm{~mm}^{2}$ for moderate, and $1.22 \mathrm{~mm}^{2} \pm 1.25 \mathrm{~mm}^{2}$ for high. The resulting average contact-localized degrees of wear were utilized moving forward as a set of three predictors for modeling of accidentals.

\section{Modeling Fitting}

After checking for multicollinearity between the predictors (for both subset contact and subset wear) using the variance-inflation factor (VIF <10) [4-6], four modeling techniques (non-spatial GLM, AP, Durbin, GWPR) were applied to evaluate spatial distributions for 27,933 features contained within 987 possible locations on a standardized outsole. In addition, three neighborhood cut-off distances $(17 \mathrm{~mm}, 34 \mathrm{~mm}, 51 \mathrm{~mm})$ were explored in order to assess differences in spatial effects and model performance as a function of scale. Therefore, there were a total of 24 models constructed as part of this proof of concept study (2 predictors of interest $\times 3$ neighborhoods $\times 4$ regression approaches). Tables $5 \& 6$ detail the estimated coefficients for all models, with mean values reported for the spatial GWPR method (summary statistics for each set of conditions for GWPR are provided in Tables A.1-A.2 in the Appendix). When considering the contribution of contact and degree of wear, both predictors yield positive associations with RAC counts, suggesting that increased contact as well as wear at a specific location likewise increases the number of features that are observed. More specifically, moderate wear has the largest impact on accidental frequency as compared to light and high. This observation fits with intuition wherein regions of light wear can develop RACs, but it is expected that fewer would be observed than in areas that contain more pressure during the process of use. Furthermore, in areas of high wear it is 

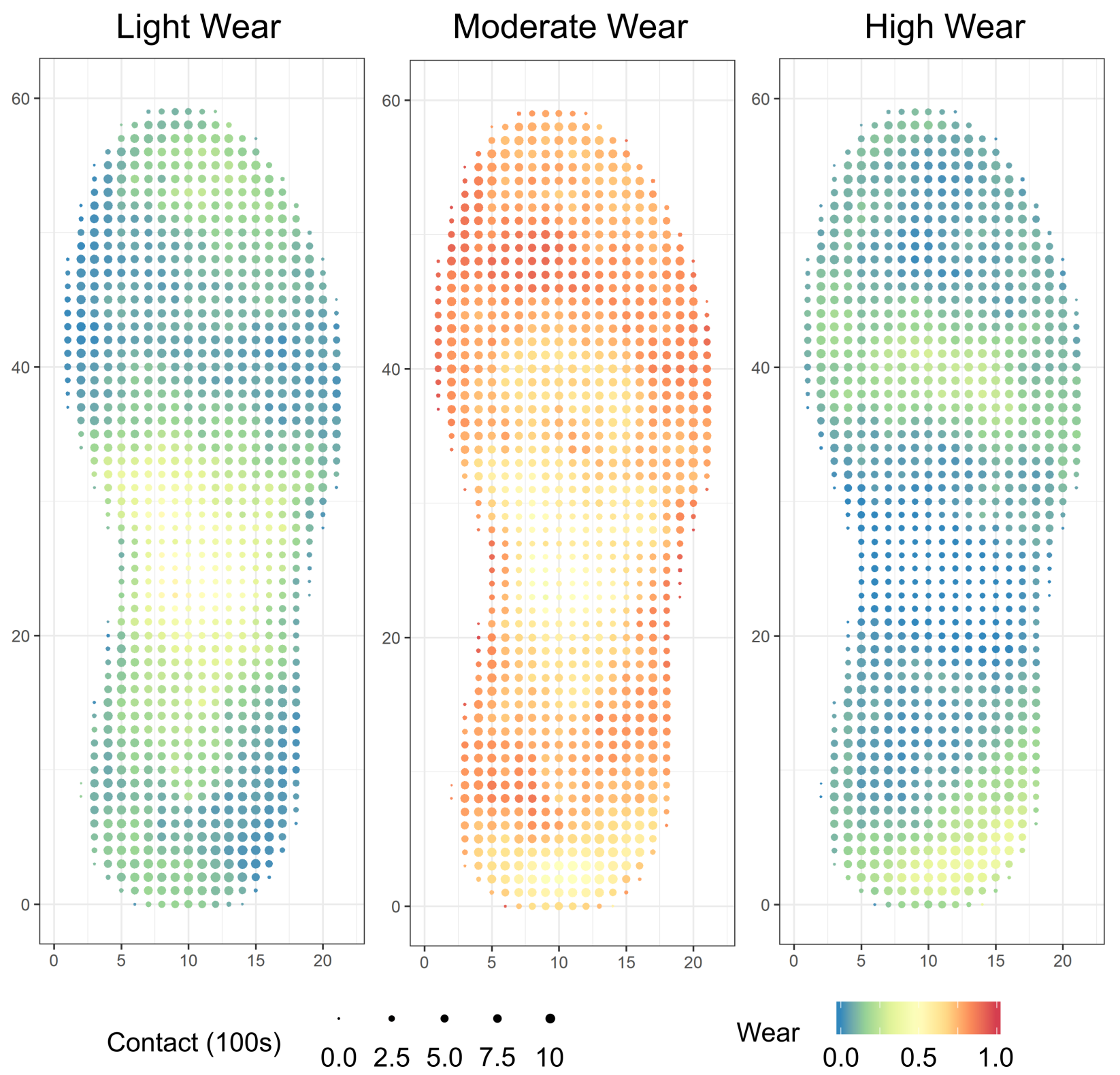

Figure 4: Distribution of average contact area (point size) and fractional wear (color) for the subset database of 130 shoes. Fractional wear represents the percentage of the contact area that was classified as each level of wear; summing across low, moderate, and high wear for a given location will yield 1 (100\% of the contact area in the cell). Also, note that average contact area is the total number of contact pixels in a given location divided by the total number of shoes and ranges from 13-10,373px (approximately $0.2-18 \mathrm{~mm}^{2}$ ).

expected that the appearance and subsequent degradation of features occurs more quickly thereby yielding a less stable association between high wear areas and accidentals.

However, comparison of model coefficients between contact and contact-localized wear 
revealed several dissimilarities, including directionality and significance among predictors. When considering the contact subset, all parameters were significantly informative for predicting the locations and counts of RACs, irrespective of the model and neighborhood size. Furthermore, all coefficients exhibited a positive impact on the number of RACs with the exception of the contact area spatial lag in the spatial Durbin model, as previously observed for the full database analysis (Table 5). Alignment between all contact models provides strong evidence of the significant positive relationship between tread area and RACs, wherein an increase in contact likewise promotes an increase in the number of features.

Table 5: Model coefficients for subset data predicting RAC counts as a function of contact area (with a bin percentage offset variable) using three different neighborhood cut-off radii. Note that the results presented for the GWPR model are the mean coefficients for each predictor across all 987 cell-specific models.

\begin{tabular}{|c|c|c|c|c|c|c|c|c|c|c|}
\hline Model & $\begin{array}{c}\text { Non-Spatial } \\
\text { GLM }\end{array}$ & & $\begin{array}{c}\text { Spatial } \\
\text { AP }\end{array}$ & & & $\begin{array}{l}\text { Spatial } \\
\text { Durbin }\end{array}$ & & & $\begin{array}{l}\text { Spatial } \\
\text { GWPR }\end{array}$ & \\
\hline Neighborhood & $N A$ & 400 & 800 & 1200 & 400 & 800 & 1200 & 400 & 800 & 1200 \\
\hline $\begin{array}{c}\text { Intercept } \\
\left(\beta_{0}\right)\end{array}$ & 2.376 & 1.842 & 1.752 & 1.653 & 1.950 & 1.916 & 1.896 & 2.598 & 2.480 & 2.296 \\
\hline $\begin{array}{c}\text { Contact } \\
\left(\beta_{1}\right)\end{array}$ & $1.6 \mathrm{E}-04$ & $9.0 \mathrm{E}-05$ & $1.3 \mathrm{E}-04$ & $1.4 \mathrm{E}-04$ & $9.5 \mathrm{E}-05$ & $1.3 \mathrm{E}-04$ & $1.5 \mathrm{E}-04$ & $1.0 \mathrm{E}-04$ & $1.4 \mathrm{E}-04$ & $1.8 \mathrm{E}-04$ \\
\hline $\begin{array}{c}\log (\operatorname{Bin}) \\
\left(\beta_{2}\right)\end{array}$ & 0.9433 & 1.044 & 1.121 & 1.124 & 0.9185 & 0.9870 & 0.9763 & 1.085 & 0.9429 & 0.8265 \\
\hline $\begin{array}{c}\text { Spatial Lag } \\
\text { RACs } \\
(\rho)\end{array}$ & NA & 0.0314 & 0.0340 & 0.0365 & 0.0327 & 0.0356 & 0.0386 & NA & NA & NA \\
\hline $\begin{array}{c}\text { Spatial Lag } \\
\text { Contact } \\
(\theta)\end{array}$ & NA & NA & NA & NA & $-6.3 \mathrm{E}-05$ & $-7.2 \mathrm{E}-05$ & $-9.2 \mathrm{E}-05$ & NA & NA & NA \\
\hline
\end{tabular}

Conversely, the same trends observed for RAC distributions as a function of tread contact do not hold for the contact-localized wear predictors. For example, light wear was not significant in the spatial Durbin models $(p=0.0981-0.7499)$ and high wear did not significantly aid in prediction for the spatial AP models $(p=0.2310-0.7590)$ and the spatial Durbin model using a neighborhood size of 1200px/51mm ( $p=0.6938)$. Regarding directionality of coefficients, the contact-localized wear models exhibit some inconsistency; contributions of light wear, high wear, and the spatial lag on high wear vary in whether there is a positive association with RAC counts between models and neighborhoods (Table 
Table 6: Model coefficients for subset data predicting RAC counts as a function of contact-localized wear (with a bin percentage offset variable) using three different neighborhood cut-off radii. Values highlighted in red indicate a non-significant predictor. Note that the results presented for the GWPR model are the mean coefficients for each predictor across all 987 cell-specific models and accordingly no assessments of predictor significance were conducted.

\begin{tabular}{|c|c|c|c|c|c|c|c|c|c|c|}
\hline \multirow{2}{*}{$\begin{array}{c}\text { Model } \\
\text { Neighborhood } \\
\end{array}$} & \multirow{2}{*}{$\begin{array}{c}\text { Non-Spatial } \\
\text { GLM } \\
N A \\
\end{array}$} & \multicolumn{3}{|c|}{$\begin{array}{c}\text { Spatial } \\
\text { AP }\end{array}$} & \multicolumn{3}{|c|}{$\begin{array}{l}\text { Spatial } \\
\text { Durbin }\end{array}$} & \multicolumn{3}{|c|}{$\begin{array}{l}\text { Spatial } \\
\text { GWPR }\end{array}$} \\
\hline & & 400 & 800 & 1200 & 400 & 800 & 1200 & 400 & 800 & 1200 \\
\hline $\begin{array}{l}\text { Light Wear } \\
\qquad\left(\beta_{1}\right)\end{array}$ & $-2.4 \mathrm{E}-04$ & $1.3 \mathrm{E}-04$ & $1.3 \mathrm{E}-04$ & $8.2 \mathrm{E}-05$ & $6.0 \mathrm{E}-05$ & $3.3 \mathrm{E}-05$ & $-9.1 \mathrm{E}-06$ & $-8.1 \mathrm{E}-05$ & $-7.3 \mathrm{E}-05$ & $-8.9 \mathrm{E}-05$ \\
\hline $\begin{array}{c}\text { Moderate Wear } \\
\qquad\left(\beta_{2}\right)\end{array}$ & $2.5 \mathrm{E}-04$ & $1.4 \mathrm{E}-04$ & $1.5 \mathrm{E}-04$ & $1.6 \mathrm{E}-04$ & $1.4 \mathrm{E}-04$ & $1.6 \mathrm{E}-04$ & $1.9 \mathrm{E}-04$ & $1.9 \mathrm{E}-04$ & $2.0 \mathrm{E}-04$ & $2.3 \mathrm{E}-04$ \\
\hline $\begin{array}{c}\text { High Wear } \\
\left(\beta_{3}\right) \\
\end{array}$ & $-1.0 \mathrm{E}-04$ & $1.3 \mathrm{E}-05$ & $3.2 \mathrm{E}-06$ & $-1.0 \mathrm{E}-05$ & $1.3 \mathrm{E}-05$ & $7.9 \mathrm{E}-05$ & $7.7 \mathrm{E}-06$ & $1.3 \mathrm{E}-05$ & $4.8 \mathrm{E}-06$ & $-9.1 \mathrm{E}-06$ \\
\hline $\begin{array}{c}\log (\operatorname{Bin}) \\
\left(\beta_{4}\right) \\
\end{array}$ & 1.162 & 0.9397 & 1.007 & 1.043 & 0.8731 & 0.9581 & 0.9588 & 1.134 & 1.027 & 0.9821 \\
\hline $\begin{array}{c}\text { Spatial Lag } \\
\text { Light } \\
\left(\theta_{1}\right)\end{array}$ & NA & NA & NA & NA & $1.0 \mathrm{E}-04$ & $2.3 \mathrm{E}-04$ & $4.2 \mathrm{E}-04$ & NA & NA & NA \\
\hline $\begin{array}{c}\text { Spatial Lag } \\
\text { Moderate } \\
\left(\theta_{2}\right)\end{array}$ & NA & NA & NA & NA & $-1.5 \mathrm{E}-05$ & $-1.7 \mathrm{E}-05$ & $-8.3 \mathrm{E}-05$ & NA & NA & NA \\
\hline $\begin{array}{c}\text { Spatial Lag } \\
\text { High } \\
\left(\theta_{3}\right)\end{array}$ & NA & NA & NA & NA & $-1.4 \mathrm{E}-04$ & $-9.6 \mathrm{E}-05$ & $5.0 \mathrm{E}-05$ & NA & NA & NA \\
\hline
\end{tabular}

6). This phenomenon, wherein different regressions and model conditions yield shifts in predictor significance and contribution, has been well documented in the literature [6-10]. This observation further reiterates the importance of evaluating multiple models in order to deduce the most appropriate technique that adequately describes the data generating structure, which was incorporated in this study using a bottom-up approach $[6,7,11,12]$.

With specific regard to this study, this observation has several implications. First, irrespective of the model selected, some variables remain important predictive factors for location-specific RAC counts, including moderate wear, bin partiality, and spatial lags for 
feature frequencies and light wear (when applicable). Therefore, there is strong evidence that each of these components are a significant factor in the data generating process for accidental distributions. In addition, the directionality of some coefficients exhibit changes when considering smaller neighborhoods $(17 \mathrm{~mm}, 34 \mathrm{~mm})$ versus longer distances $(51 \mathrm{~mm})$, as detailed in Table 6 . The same is true for predictor significance, wherein significance changes at the $51 \mathrm{~mm}$ radius. Considered together, these results suggest that the influence of these coefficients (e.g., high wear and the spatial lag for high wear) affect RAC counts differently at different spatial scales.

Ultimately, consideration of the regression models estimated using contact-localized wear as a predictor reveals three major results. First, moderate wear is the most informative and consistent predictor for RAC distributions, as compared to light and high degrees of wear. Across all neighborhoods and techniques, this is the only wear factor that remains significant, does not change directionality (positive association with RAC counts), and imparts the largest effect (Table 6). Second, light and high wear exhibit large variability in the way they influence feature counts depending on the model and distance of interest. For example, light wear exhibits a significant positive effect on RAC counts when using the spatial AP model, a significant negative effect using the spatial GWPR model, and no significant effect using the spatial Durbin model. This observation may be tied in with the expected variability in RAC counts for light wear, wherein activity could be an underlying confounder; however, this could also be attributed to purely mathematical fitting. Third, the data in Table 6 provide evidence that scale dependent spatial effects exist in RAC distributions. These observations involve a change in effect between shorter neighborhood distances $(400 \mathrm{px} / 17 \mathrm{~mm}, 800 \mathrm{px} / 34 \mathrm{~mm})$ and the longer radius $(1200 \mathrm{px} / 51 \mathrm{~mm})$. Therefore, when evaluating RAC distributions scale must be considered. In order to evaluate whether each of these factors can be attributed to a practical consideration or a mathematical artifact, additional analyses would be required to increase the sample size and determine whether this result persists. Moreover, this proof of concept study utilized just $10 \%$ of the WVU footwear database, but this sample also contained nearly $40 \%$ of all RACs. Therefore, given the relatively high concentration of accidentals in this subset, it is important to evaluate how addition of more shoes, each containing fewer features, impacts these observations. 


\section{Evaluation of Model Residuals}

In an effort to assess the appropriateness of each model and determine whether there still remains an unmodeled factor that impacts the spatial distribution of RACs after accounting for wear, analysis of model residuals using Moran's I was conducted as previously described. Pearson residual plots for modeling accidentals as a function of contact area (Figures A.3A.5) and contact-localized wear (Figures A.6-A.8) at three different neighborhood cut-off radii are available in the Appendix. With respect to the non-spatial GLM, all of the spatial models show an improvement in prediction, with more residuals near 0 and fewer at the extremes, irrespective of predictor (contact vs wear) and neighborhood. Thus, as previously determined for the contact area data on the full database, there remains evidence of spatial effects that impact RAC distributions across the 130 shoe subset. Likewise, all models yielded significant positive global spatial autocorrelation according to the resulting Moran's I statistics. However, the global Moran's I values for contact-localized wear were lower than those obtained using contact as a predictor, as detailed in Table 7. On average, the global I statistic exhibited a $33.7 \% \pm 10.4 \%$ decrease when comparing the residuals resulting from models built using the same regression method and neighborhood size between contact and contact-localized wear.

Table 7: Global and local Moran's I results for residuals of RACs predicted as a function of contact area or contact-localized wear. Local Moran's I results indicate the percentage of cells exhibiting significant local residual spatial correlation.

\begin{tabular}{|c|c|c|c|c|c|c|c|c|c|c|c|c|c|}
\hline \multicolumn{2}{|c|}{ Model } & \multicolumn{3}{|c|}{$\begin{array}{c}\text { Non-Spatial } \\
\text { GLM }\end{array}$} & \multicolumn{3}{|c|}{$\begin{array}{c}\text { Spatial } \\
\text { AP }\end{array}$} & \multicolumn{3}{|c|}{$\begin{array}{l}\text { Spatial } \\
\text { Durbin }\end{array}$} & \multicolumn{3}{|c|}{$\begin{array}{l}\text { Spatial } \\
\text { GWPR }\end{array}$} \\
\hline \multicolumn{2}{|c|}{ Neighborhood } & 400 & 800 & 1200 & 400 & 800 & 1200 & 400 & 800 & 1200 & 400 & 800 & 1200 \\
\hline \multirow{2}{*}{ Contact } & Global & 0.637 & 0.555 & 0.483 & 0.133 & 0.120 & 0.104 & 0.121 & 0.109 & 0.097 & 0.111 & 0.166 & 0.165 \\
\hline & Local & $32 \%$ & $40 \%$ & $48 \%$ & $10 \%$ & $12 \%$ & $14 \%$ & $9.5 \%$ & $11 \%$ & $11 \%$ & $8.6 \%$ & $17 \%$ & $20 \%$ \\
\hline \multirow{2}{*}{ Wear } & Global & 0.445 & 0.356 & 0.308 & 0.094 & 0.088 & 0.086 & 0.085 & 0.080 & 0.072 & 0.054 & 0.086 & 0.087 \\
\hline & Local & $32 \%$ & $42 \%$ & $46 \%$ & $6.9 \%$ & $9.4 \%$ & $13 \%$ & $6.6 \%$ & $8.4 \%$ & $10 \%$ & $4.5 \%$ & $8.8 \%$ & $14 \%$ \\
\hline
\end{tabular}

Following global evaluation of spatial autocorrelation, local estimates of Moran's I were likewise computed for the residuals of each model, as detailed in Table 7. For each of the 24 models, the percentage of locations exhibiting significant residual spatial associations was recorded. Figure 5 illustrates the distribution of local Moran's I results for the most optimal 
models at each neighborhood cut-off, defined as the model resulting in the fewest cells containing significant spatial association. In addition, all local spatial autocorrelation plots are provided in the Appendix (Figures A.9-A.14). In alignment with the observations made for the full database, the vast majority of significant correlations were positive (red dots), with few cells for each model exhibiting an inverse association with their neighbors (blue dots). Again, the non-spatial GLM produced the largest areas of localized spatial correlation as well as minimal difference between contact and contact-localized wear predictors, with $32 \%$ $48 \%$ of locations yielding significant Moran's I values (Table 7). Moreover, the same trend observed for the global Moran's I analysis was obtained for local evaluations. As detailed in Table 7, using contact-localized wear as a predictor decreased the degree of local autocorrelation as compared to contact area alone, with an average of just $9.1 \% \pm 3.0 \%$ (versus $13 \% \pm$ $3.8 \%$ ) significant locations across all spatial models and neighborhood cut-off radii. In order to assess whether wear statistically improved model predictions based upon local Moran's I results, McNemar's test $[4,13,14]$ was utilized to compare the proportion of cells exhibiting significant residual correlations. More specifically, for each neighborhood size, contact and wear results were compared for the regression exhibiting the best performance (GWPR for 400px/17mm and Durbin for $800 \mathrm{px} / 34 \mathrm{~mm}$ and $1,200 \mathrm{px} / 51 \mathrm{~mm}$ as detailed in Table 7 ). The statistical analysis revealed that wear exhibited fewer cells with persisting spatial correlation $\left(p=2.1 \times 10^{-6}, 4.6 \times 10^{-2}\right.$, and $4.9 \times 10^{-2}$ for $17 \mathrm{~mm}, 34 \mathrm{~mm}$, and $51 \mathrm{~mm}$, respectively) . However, it should be acknowledged that the $p$-values for the two larger neighborhood sizes are very close to $\alpha=0.05$, and should therefore be interpreted with caution. In other words, while these $p$-values are indicate that the difference in the proportion of significant cells is more than expected by chance, there is only weak support of a statistically significant difference and thus contact-localized wear only offered an incremental improvement over contact alone. This observation makes sense because contact-area was able to well describe RAC distributions across the majority of the outsole, with just three localized areas in the ball of the toe, arch, and edge of the heel that required wear to improve prediction.

Overall, the results of the residual assessments indicate that using contact-localized wear as a predictor (the intersection of tread contact and gait) better accounts for spatial associations underlying RAC distributions as compared to tread contact alone, at least statistically. 
GWPR (400px)

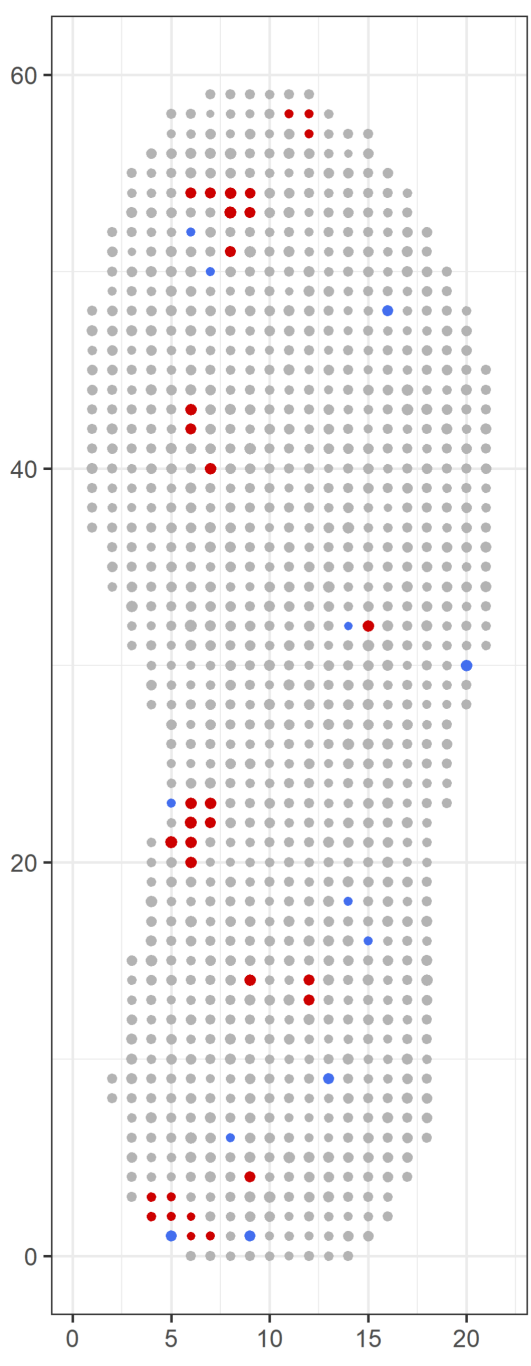

Residuals
Durbin (800px)

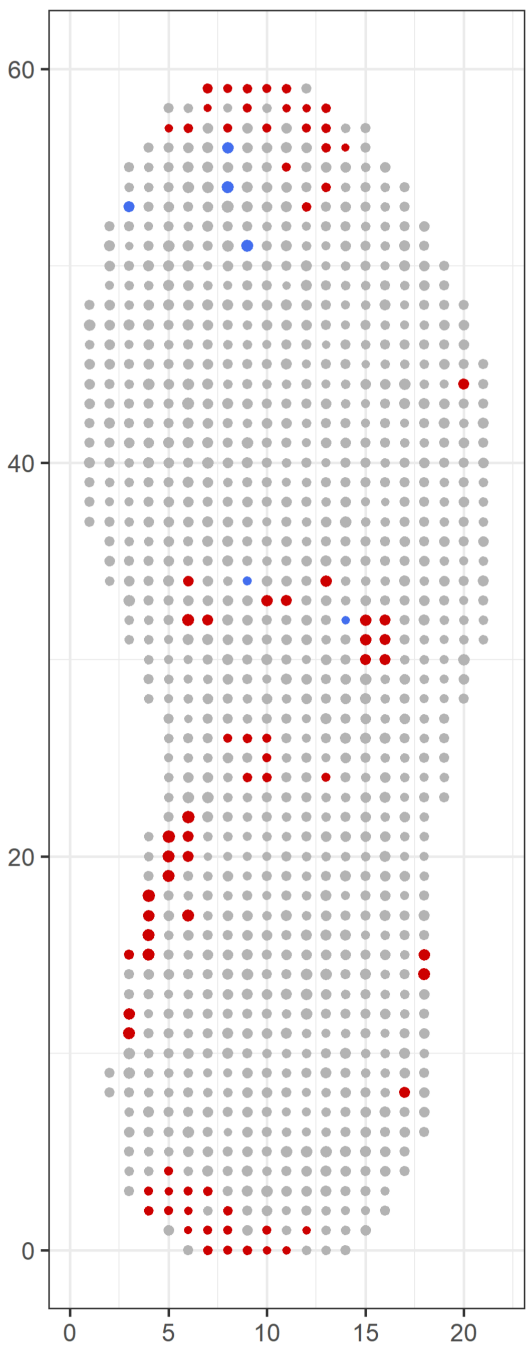

Durbin (1200px)

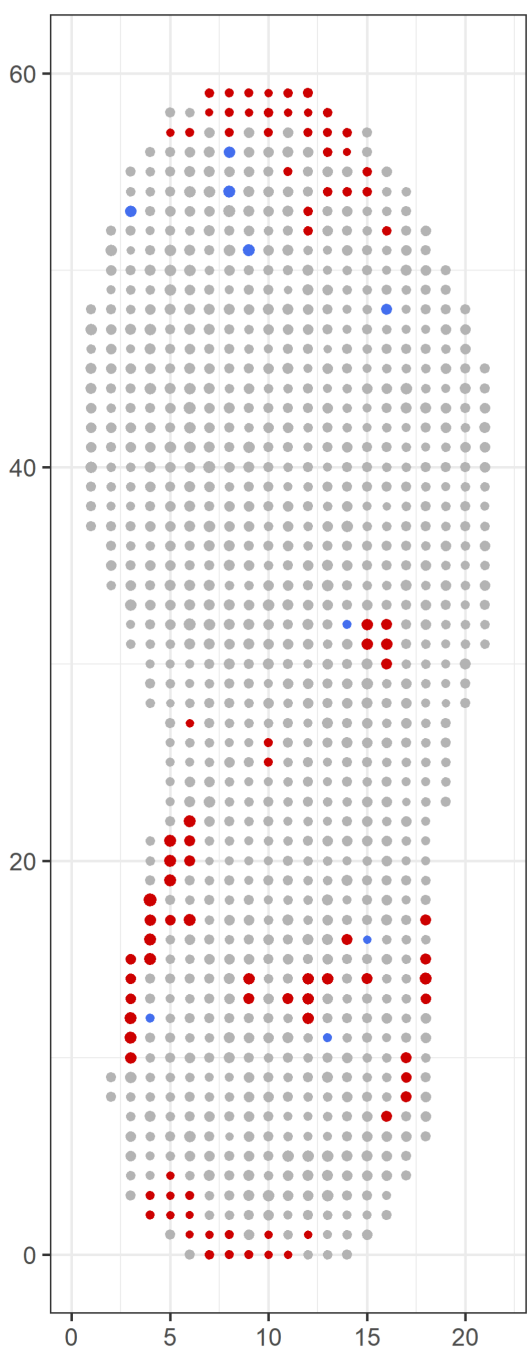

Correlation
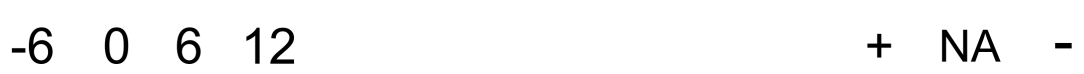

Figure 5: Spatial correlation detected (red: positive, blue: negative, gray: none) and associated Pearson residuals (size) of RAC counts predicted as a function of contact-localized wear (with a bin percentage offset) for the subset database of 130 shoes. Results are displayed for the model resulting in the fewest cells exhibiting significant local autocorrelation at each neighborhood cut-off radius (400px/17mm, 800px/34mm, $1200 \mathrm{px} / 51 \mathrm{~mm})$. Note that for all neighborhood sizes, wear models outperformed contact-only models with respect to the percentage of locations exhibiting significant local residual correlation.

The previous analysis of accidentals as a function of contact, using the full database, revealed two major conclusions. First, spatial effects are a significant factor that must be incorporated 
in characterization of randomly acquired characteristics. This outcome is further supported by this proof of concept study of both contact and wear at various scales. Namely, the global Moran's I scores for the non-spatial GLM model are again 3-5 times larger than those obtained for the spatial models, regardless of the predictor and neighborhood size. Second, the results obtained from the full database study using contact area as an explanatory factor for locations/frequencies of RACs indicated that an important factor remained unmodeled, with $22-13 \%$ of cells exhibiting residual correlations. Based upon previous observations of the regions of significant local autocorrelation, it was hypothesized that wear could be one such variable. The results of this proof of concept study indicate that wear does, in fact, improve predictions as evidenced by the lower proportion of locations exhibiting significant residual correlation, specifically at the lug level of $400 \mathrm{px} / 17 \mathrm{~mm}$ (just $4.5 \%$ of locations were insufficiently predicted). Considered practically, the observed performance gains, although mathematically informative, are relatively minor for this proof of concept database. Moreover, greater than $90 \%$ of the all positions on the outsole are well modeled, irrespective of the predictor and assuming that GWPR is used for the 400px/17mm neighborhood and Durbin is used for the $800 \mathrm{px} / 34 \mathrm{~mm}$ and $1,200 \mathrm{px} / 51 \mathrm{~mm}$ radius. To determine if this trend holds, the database should be expanded to include more shoes, with additional variations in RAC counts and potentially the locations of wear.

\section{Model Assessment and Selection}

The performance of all 24 models was again compared using mean absolute deviation (MAD), mean squared error (MSE), and Akaike information criteria (AIC), as previously described. Table 8 reports the resulting model assessment statistics for each of the regressions estimating RAC count as a function of either contact or contact-localized wear for the proof of concept study. Models were compared across regression techniques and both predictors within the same cut-off radius, thus yielding a description of the optimal model based upon the neighborhood size of interest. Overall, all wear-informed models outperformed the associated contact models, further enforcing the results and observations made using the residual analysis. Furthermore, all models produced the best predictions at the 400px $(17 \mathrm{~mm})$ neighborhood size, as evidenced by the lowest values of all statistics within 
each condition (Table 8). Given that this distance is the mean lug size within the database, these results indicate that RACs distributions are best characterized when evaluated with respect to the same tread element. This observation fits with intuition because it is expected that highly similar contact areas and degree of wear would exist within a single lug, whereas these factors are expected to vary more between elements. Not surprisingly then, the overall best model to describe accidentals was the GWPR model that utilized wear as a predictor and applied a 400px (17mm) neighborhood cut-off, as indicated by the green cells in Table 8. This result suggests that the global models are unable to capture spatial associations and heterogeneity in small neighborhoods and hence, the local regression (which estimates a model for each location) better describes the data.

In contrast, the spatial Durbin model, estimated using contact-localized degree of wear, was optimal for both of the larger neighborhood sizes (yellow and blue cells in Table 8). The Durbin model is the most robust model in the presence of spatial correlation because it allows for incorporation of both endogenous and exogenous interactions. By utilizing spatial lag variables of the responses and predictors, wherein the values of neighboring cells' contactlocalized wear and feature counts are used to aid in predicting RAC counts, the Durbin model produces the most accurate predictions, even with increased variability between variables at larger distances. In contrast, the GWPR approach is the weakest of all spatial models at larger cut-off radii, indicating that estimates produced are too localized and performance suffers given that there is no global spatial lag term(s).

Overall, the trends observed for model performance in this study are in alignment with those previously published in the literature. More specifically, GWPR performs best within smaller neighborhoods and alternatively, global spatial models exhibit better prediction for larger radii $[6,15,16]$. Given that GWPR estimates location-specific models, it is innately well suited to capture local variations in relationships between predictors and responses $[6,15$, 16]. In other words, within small neighborhoods, this approach is able to precisely estimate coefficients, due to the relatively low bias. Thus, the model produces accurate, localized predictions that exhibit large variation across the spatial region $[6,15,16]$. Conversely, at larger distances the local GWPR models are estimated by averaging observed relationships over more area, thereby increasing bias and yielding less variable coefficients within the 
Table 8: Model assessment statistics describing model fits for RAC counts as a function of contact area or contact-localized wear, with a bin percentage offset variable to adjust for partiality. The green cells reflect the best fit model for the $400 \mathrm{px} / 17 \mathrm{~mm}$ neighborhood (and also the overall optimal model). The yellow cells reflect the best fit model for the $800 \mathrm{px} / 34 \mathrm{~mm}$ neighborhood. The blue cells reflect the best fit model for the 1200px/51mm neighborhood. All L(AICs) were computed with respect to the best fit model for the specified neighborhood size. Note: an L(AIC) value of Inf in the table represents $\mathrm{L}(\mathrm{AIC})>10^{305}$.

\begin{tabular}{|c|c|c|c|c|c|c|c|c|c|c|c|}
\hline \multirow{2}{*}{\multicolumn{2}{|c|}{$\begin{array}{c}\text { Model } \\
\text { Neighborhood }\end{array}$}} & \multirow{3}{*}{$\begin{array}{c}\text { Non-Spatial } \\
\text { GLM } \\
N A \\
9.388 \\
\end{array}$} & \multicolumn{3}{|c|}{$\begin{array}{c}\text { Spatial } \\
\mathbf{A P}\end{array}$} & \multicolumn{3}{|c|}{$\begin{array}{l}\text { Spatial } \\
\text { Durbin }\end{array}$} & \multicolumn{3}{|c|}{$\begin{array}{l}\text { Spatial } \\
\text { GWPR }\end{array}$} \\
\hline & & & \multirow{2}{*}{$\frac{400}{5.567}$} & \multirow{2}{*}{$\begin{array}{c}800 \\
5.716\end{array}$} & \multirow{2}{*}{$\begin{array}{l}1200 \\
5.917 \\
\end{array}$} & \multirow{2}{*}{$\begin{array}{c}400 \\
5.486 \\
\end{array}$} & \multirow{2}{*}{$\begin{array}{c}800 \\
5.656\end{array}$} & \multirow{2}{*}{$\begin{array}{l}1200 \\
5.875\end{array}$} & \multirow{2}{*}{$\begin{array}{c}400 \\
5.297 \\
\end{array}$} & \multirow{2}{*}{$\begin{array}{c}800 \\
6.198\end{array}$} & \multirow{2}{*}{$\begin{array}{l}1200 \\
6.659 \\
\end{array}$} \\
\hline \multirow{4}{*}{ Contact } & MAD & & & & & & & & & & \\
\hline & MSE & 153.7 & 53.71 & 56.79 & 61.13 & 52.38 & 55.99 & 60.31 & 49.55 & 68.52 & 80.18 \\
\hline & $\mathrm{AIC}$ & 9883 & 6731 & 6903 & 7088 & 6696 & 6866 & 7040 & 6556 & 7162 & 7550 \\
\hline & $\mathrm{L}(\mathrm{AIC})$ & Inf & $2.2 \mathrm{E} 85$ & $5.6 \mathrm{E} 29$ & 3.3E39 & $5.5 \mathrm{E} 77$ & $5.2 \mathrm{E} 21$ & $1.3 \mathrm{E} 29$ & $2.2 \mathrm{E} 47$ & $9.8 \mathrm{E} 85$ & 7.0E139 \\
\hline \multirow{4}{*}{ Wear } & MAD & 7.903 & 5.436 & 5.556 & 5.713 & 5.407 & 5.555 & 5.691 & 4.993 & 5.707 & 6.130 \\
\hline & MSE & 103.9 & 51.27 & 53.88 & 57.58 & 50.74 & 53.58 & 56.81 & 43.82 & 56.86 & 64.05 \\
\hline & $\mathrm{AIC}$ & 8411 & 6644 & 6788 & 6942 & 6627 & 6766 & 6906 & 6338 & 6830 & 7108 \\
\hline & $\mathrm{L}(\mathrm{AIC})$ & Inf & $2.8 \mathrm{E} 66$ & $6.0 \mathrm{E} 04$ & $6.6 \mathrm{E} 07$ & $5.7 \mathrm{E} 62$ & 1.00 & 1.00 & 1.00 & $7.9 \mathrm{E} 13$ & $7.3 \mathrm{E} 43$ \\
\hline
\end{tabular}

study region which essentially creates a smoothing effect $[6,15,16]$. Tables A.1 \& A.2 offers evidence of this trend wherein the range of each coefficient is largest at $400 \mathrm{px} / 17 \mathrm{~mm}$ and decreases with each increase in neighborhood cut-off radius. Therefore, when evaluating RAC distributions and incorporating spatial effects at larger distances, the global models, which produce single estimates of each coefficient, are better able to model the relationships between features and contact or contact-localized wear.

\section{Conclusions}

The goal of this study was to investigate and characterize RAC distributions in order to inform weight of evidence assessments for footwear source attribution comparisons and decisions. In pursuit of this objective, this research applied commonly-used spatial regression techniques in order to understand the "data-generating process" of feature locations and frequencies. The major benefits of using these techniques are four-fold. First, implementation of various regression approaches, each considering two predictors and three neighborhood sizes, allowed for the systematic determination of the optimal model to describe RAC distributions. Based upon the results of this proof of concept study, acquired features are 
best modeled using contact-localized degree of wear, with spatial associations considered in small neighborhoods $(400 \mathrm{px} / 17 \mathrm{~mm}$, or the average tread element diameter). Moreover, the globally-weighted Poisson regression produced the best estimates of RAC counts within these small neighborhoods, likely because it is a local technique which estimates an individual model for each location thereby accounting for localized heterogeneity of variables (Table 8).

Second, examination of model coefficients allows for an interpretation of the response of interest with respect to the magnitude and direction of relevant explanatory variables. More specifically, the number of features observed within a given location increases as both contact area and contact-localized wear increases (Tables $5 \& 6$ ). In terms of wear, moderate wear has the largest positive impact on RAC counts and persists as a significant predictor in all models. Conversely, light and high wear each impart a smaller positive effect on feature frequencies, and these categories may not offer significant contributions depending on the model and neighborhood of interest.

Third, spatial regression modeling techniques allow for incorporation of spatial dependencies, wherein the strength and direction of correlation between predictors and/or responses and their neighbors can be assessed. Regarding RAC distributions, spatial modeling produced much better estimates of the locations and frequencies of features than traditional non-spatial regression, as evidenced by the large decrease in both global and local Moran's I coefficients (Table 7). This result provides evidence that spatial effects do in fact influence the distribution of features on outsoles. In addition, spatial associations were detected for both RAC counts and contact/wear between neighboring locations, as supported by the significant contributions of their spatial lag variables, with some exceptions for degree of wear ( $\rho$ and $\theta$, respectively in Tables $5 \& 6$ ).

Lastly, investigation of multiple neighborhood sizes allow for an understanding of the underlying connectivity structure and determination of the optimal distance at which variable correlations should be considered. The results from the proof of concept study indicated that RACs are best predicted when allowing for spatial correlations within neighborhood sizes representing the average lug diameter $(400 \mathrm{px} / 17 \mathrm{~mm})$. As detailed in Table 8 , each spatial model performed best using a cut-off radius of 400px, with each successive neighborhood 
increase yielding worse predictions. This result matches intuition that outsole conditions are likely very similar within one tread element, and become increasingly more variable with increased distance.

Considered collectively, the results indicate that the data-generating process of RACs with respect to contact-localized wear is likely scale dependent. This conclusion is strongly supported by the change in optimal model as a function of neighborhood size, as well as the observed variability in significance and direction of coefficient effects between smaller and larger distances. As previously discussed, local modeling via GWPR exhibited the highest performance at the lug level $(400 \mathrm{px} / 17 \mathrm{~mm})$, as well as the highest performance overall for predicting RAC counts. Hence, heterogeneity in feature frequencies as well as degree of contact-localized wear seems to be present within small neighborhoods. However, this outcome does not preclude the presence of spatial correlation as well. Although GWPR does not directly incorporate spatial associations (as spatial lag predictors), it does explicitly consider location; therefore, it has been shown to likewise reduce existing spatial correlation locally [16]. Not surprisingly, at larger distances global modeling using a Durbin regression, which includes both exogenous and endogenous effects and was shown to be optimal for the full database evaluation conducted in Part I, maximizes prediction accuracy. Again, this result does not indicate that heterogeneity is lacking in larger neighborhoods, rather that consideration of spatial correlations are sufficient.

While this study was successfully able to characterize several factors impacting the distribution of acquired features on outsoles, there were several limitations that must be noted. As previously discussed in Chapters 2 and 3, the tread contact maps were created using an automated image processing procedure wherein several morphological operators were applied to Handiprint images in order to segment contact pixels. While every effort was made to ensure high quality binary maps, the Handiprint images are inherently speckled given that they are created using black powder, and this fact precludes the production of perfect tread contact representations. A more supervised process, such as using a high resolution outsole scanner and/or manually annotating or modifying tread contact regions, may yield more accurate maps. However, this process would be both time- and cost- intensive and may not provide significant changes or improvements to the results obtained for this study to warrant 
the investment.

Additionally, the wear maps were created through systematic evaluation of outsole and exemplar images and subsequent marking by a single analyst. Prior to starting this process on the proof of concept database, this researcher completed a two and a half day training program which included relevant background readings, discussion of wear criteria, and numerous classification tasks (on both localized regions and full maps). While every effort was afforded to maximize consistency in applying classification rules and subsequent identification and marking of wear regions, this task is inherently subjective. As such, it is reasonable to expect that intra- and inter-analyst variability exits and that if the same subset data were re-analyzed at a different time or by a different examiner, some variation in regions of wear would exist. However, while these differences would almost certainly yield come variability in specific coefficients and summary statistics, it is not expected that this would impart significant changes into the overall observations and conclusions.

Furthermore, all contact and wear maps were poly-warped to allow for inter-comparison of the entire WVU database of shoes. While this was a necessary step to increase the sample size available for analysis, this process likewise limits the utility of the results owing to the induced modification of tread appearance. To overcome this limitation, a database would need to be constructed that contains a large variety of outsoles each with numerous replicates to allow for examination within the same class characteristics. However, this undertaking would likely be difficult to implement in practicality given the cost, time, and resources associated with compiling such a large collection of shoes. Furthermore, it would be necessary to constantly expand the database as new outsole designs are produced, which could become prohibitive.

When considering the conclusions obtained in this study, it is likewise important to address several data simplifications associated with the observed results. First, this study only investigated the effects of two outsole related factors (tread contact and contact-localized wear). Hence, any other observables across the shoe, such as outsole material and the specific locations of Schallamach patterns, were ignored and essentially considered equal. Therefore, any differences in the rate of RAC development, say between a soft microcellular material and a harder rubber material, were not included in the model. Moreover, Schallamach patterns 
are considered to be "specific wear," providing increased discrimination potential; one study even found that a Schallamach pattern as small as $2 \mathrm{~mm} \times 3 \mathrm{~mm}$ could be sufficient to reach an identification of source [17]. While omission of these factors allowed for a more directed proof of concept study, this may also limit the ability of a model to capture the sources of some variation as a function of additional outsole conditions.

In addition, the categorization of wear was considered using three broad classes- light, moderate, and high. This classification scheme likely over-simplifies the nuances within levels of wear. For example, consider two cases of high wear. In the first case, the region exhibits complete balding, but has not worn so far as to cause a hole or tear. In the second case, a specific area of the outsole has degraded so significantly, that a large hole develops. In the latter scenario, it is expected that more accidentals may be present, owing to the fact that the hole itself would be identified as a discriminating RAC that is expected to persist over time. In contrast, the former scenario may be expected to have fewer accidentals because the bald region suggests that features appear and disappear rapidly. While additional levels and/or subcategories could be incorporated into the wear classification criteria, this expansion would also likely increase variability in identification and marking of wear regions which may actually decrease the utility of the resulting models.

Despite the limitations and simplifications outlined above, the results from the proof of concept study are extremely promising and exhibit high model performance for the prediction of RAC locations and frequencies using contact-localized degree of wear. Namely, just 4.5\% $8.5 \%$ of cells exhibit residual spatial correlation for the optimal model at each neighborhood cut-off (Table 7). This result suggests that the regressions are able to adequately account for the variation and association between responses and predictors across more than $90 \%$ of the outsole surface, with only the medial heel, arch, and the edges of the toe and heel exhibiting residual spatial correlation. Furthermore, this study specifically evaluated two predictors of interest that were reasonably expected to impact RAC distributions, but that were also readily observable on outsoles. It is important to consider, however, that there is some level of variability that cannot realistically be characterized by patent features alone, and relate more to individual properties of use. For example, two outsoles could both be classified globally as exhibiting high wear, wherein there is widespread balding/loss of texture and 
degradation of tread elements. However, suppose the owner of one shoe is a runner who consistently uses his/her shoes on gravel paths and the other is a nurse who wears his/her shoes extensively over tiled floors. The runner's outsoles would be expected to contain many more RACs than the nurse, simply owing to the substrate over which the shoes are worn. Conversely, the same runner could purchase a brand new pair of shoes and wear them running once. While the overall degree of outsole wear would be light, there would likely be numerous observable accidentals, which may not be expected for the wear level. Unarguably, incorporation of some of these more nuanced latent use variables would likely improve model predictions. However, when building a model to describe RAC distributions, a balance must be reached between hyper-specificity (wherein features are described using any and all available/useful predictors) and model usability. In other words, for examiners to realistically implement considerations of RAC distributions and weight of evidence, it is imperative that the variables used to characterize features be patent on outsoles and readily transferrable to crime scene impressions so that they can be incorporated into the comparison process.

The purpose of this study was to investigate RAC spatial distributions on outsoles using common spatial regression techniques on a large-scale database in order to inform weight of evidence. More specifically, one of the major considerations for weight of evidence is whether features are associated in space, which must be understood in order to properly ascribe value to observed similarities between suspect shoes and evidence impressions. Using a non-spatial model, which treats all observations the same irrespective of location, results in significant residual correlations in 32\%-48\% of cells (with a mean of $40 \% \pm 6.8 \%$ ), depending on predictor and neighborhood size. Under the best case scenario, when cell-specific contact and neighboring feature frequencies are used to predict RAC counts, the fraction of locations exhibiting significant residual correlation is reduced by $73 \%$ (32\% versus $8.6 \%$ for non-spatial GLM and GWPR, respectively). Likewise, when cell-specific contact-localized wear and neighboring feature frequencies are used to predict RAC counts, the fraction of locations exhibiting significant residual correlation is reduced by $86 \%$ (32\% versus $4.5 \%$ for non-spatial GLM and GWPR, respectively). Irrespective of predictor, the optimal model was the GWPR regression using a 400px/17mm neighborhood. 
Ultimately, this work sought to characterize accidental distributions in an effort to inform more appropriate weight of evidence assignments for observed similarities between features. In considering the results collectively, there is evidence that acquired characteristics do not necessarily conform to spatial randomness, when considering their frequencies and locations alone. More specifically, one of the major criteria for spatial randomness is that observations are equally likely to occur anywhere across an entire region of interest and should therefore exhibit uniform point intensity within the space. Under this assumption, then, it would be expected that a non-spatial model, wherein location is disregarded, would be able to accurately predict accidental frequencies. For footwear evidence, this concept was updated to allow for non-uniformity due to tread contact and/or contact-localized wear by modeling features as a function of the degree of each predictor. However, the results from this study indicate that neighborhood information regarding feature counts and tread contact/contactlocalized wear are required in order to adequately model the empirical data, as evidenced by large performance increases when including spatial information (73-86\% under optimal conditions). Thus, based upon these findings, spatial considerations must be incorporated into evidentiary value assessments for acquired features. For example, an observed association between two RACs in an area with higher feature frequencies (such as the ball of the toe) are less rare based upon count alone (disregarding geometry considerations), and should be therefore ascribed less weight. Conversely, observed similarity between features located in the arch of the shoe, where there features are less dense, should be valued more heavily.

Additionally, while the spatial models provided evidence for statistically significant correlation between neighboring cells' RAC counts, this does not indicate that there is necessarily dependence between features (and this was not tested as part of this study). In other words, these results do not suggest that the presence of an accidental promotes or deters another feature from forming. Rather, this association is most likely imparted by association in the underlying outsole conditions (contact and degree of wear), with significant spatial correlations detected between nearby predictor values as well $(\rho)$. This result reasonably fits with intuition wherein similar levels of wear (and degree of contact) within localized regions thus yield similar numbers of features in locations within the area of individual tread elements. Furthermore, it should be noted that the existence of this spatial association between ac- 
cidental locations and outsole conditions does not preclude the utility of these features for informing source attribution decisions. In fact, numerous features in pattern comparisons are known to exhibit spatial dependency, yet this does not necessarily complicate or limit their value. For example, Schallamach patterns in footwear are known to contain orientation dependence, forming perpendicular to the direction of motion, yet these characteristics are considered "specific wear" and can be used to reach strong associative conclusions according to the SWGTREAD conclusion scale [18]. Additionally, the value of fingerprint minutia also exhibit spatial dependence that must be considered for weight of evidence. Due to pattern force, numerous minutia are expected to exist near the core and/or delta of a fingerprint, owing to limited available space that consequently forces ridges to terminate [19, 20]. Hence, an association in minutiae in these regions provide less discriminating value than one near the edges of the print.

Although the results indicated some regions of non-uniformity in observed feature frequencies, $68 \%$ of the outsole regions were well explained by contact area or contact-localized wear alone at the lug level, and did not require location information to adequately model RAC counts. Moreover, the positions that were not well predicted were highly localized in the ball of the toe, the arch, and the edge to mid-heel, as displayed in Figures A.9 \& A.12. Incorporation of spatial effects between neighboring RAC counts and degree of contact resulted in approximately $91 \%$ of all outsole locations being sufficiently predicted. Thus, by allowing for spatial association, contact area predictions described an additional $23 \%$ of the spatial region. Finally, prediction performance was maximized at nearly $96 \%$ by intersecting contact area with localized degree of wear. Although this increase over contact area alone is small in magnitude, there is likely some degree of confounding associated with this result. Moreover, accidental frequencies in most locations of the shoe were well predicted by contact area, but areas exhibiting additional density of high (e.g., ball of the toe) or low (e.g., arch) degrees of wear required additional information. Ultimately, the results from this proof of concept study are extremely promising and provide fundamental knowledge about the practical and theoretical/statistical factors that underpin the spatial distributions of RACs. 


\section{References}

[1] NAS, Strengthening Forensic Science in the United States: A Path Forward; Committee on Identifying the Needs of the Forensic Sciences Community, National Research Council, Technical Report, (https://www.ncjrs.gov/pdffiles1/nij/grants/228091.pdf), 2009.

[2] President's Council of Advisors on Science and Technology, Report to the President: Forensic Science in Criminal Courts: Ensuring Scientific Validity of FeatureComparison Methods, Technical Report, (https://obamawhitehouse.archives.gov/ sites/default/files/microsites/ostp/PCAST/pcast_forensic_science_report_ final.pdf), 2016.

[3] J. Speir, N. Richetelli, M. Fagert, M. Hite, W. J. Bodziak, Technical note: Quantifying randomly acquired characteristics on outsoles in terms of shape and position, Forensic Science International 266 (2016) 399-411.

[4] R. J. Freund, W. J. Wilson, D. L. Mohr, Statistical Methods, Academic Press, 3rd edition, 2010.

[5] P. Simões, I. Natário, Spatial econometric approaches for count data: An overview and new directions, International Journal of Social, Behavioral, Eduational, Economic, Business and Industrial Engineering 10(1) (2016) 348-357.

[6] Z. Ma, B. Zuckerberg, W. Porter, L. Zhang, Spatial Poisson models for examining the influence of climate and land cover pattern on bird species richness, Forest Science 58(1) (2012) 61-74.

[7] C. F. Dormann, J. M. McPherson, M. B. Araújo, R. Bivand, J. Bolliger, G. Carl, R. G. Davies, A. Hirzel, W. Jetz, W. D. Kissling, I. Khn, R. Ohlemller, P. R. Peres-Neto, B. Reineking, B. Schrder, F. M. Schurr, R. Wilson, Methods to account for spatial autocorrelation in the analysis of species distributional data: a review, Ecography 30(5) (2007) 609-628.

[8] L. M. Bini, J. A. F. Diniz-Filho, T. F. L. V. B. Rangel, T. S. B. Akre, R. G. Albaladejo, F. S. Albuquerque, A. Aparicio, M. B. Araújo, A. Baselga, J. Beck, M. I. 
Bellocq, K. Bhning-Gaese, P. A. V. Borges, I. Castro-Parga, V. K. Chey, S. L. Chown, J. Paulo de Marco, D. S. Dobkin, D. Ferrer-Castán, R. Field, J. Filloy, E. Fleishman, J. F. Gómez, J. Hortal, J. B. Iverson, J. T. Kerr, W. D. Kissling, I. J. Kitching, J. L. León-Cortés, J. M. Lobo, D. Montoya, I. Morales-Castilla, J. C. Moreno, T. Oberdorff, M. Á. Olalla-Tárraga, J. G. Pausas, H. Qian, C. Rahbek, M. Á. Rodríguez, M. Rueda, A. Ruggiero, P. Sackmann, N. J. Sanders, L. C. Terribile, O. R. Vetaas, B. A. Hawkins, Coefficient shifts in geographical ecology: an empirical evaluation of spatial and nonspatial regression, Ecography 32(2) (2009) 193-204.

[9] J. J. Lennon, Red-shifts and red herrings in geographical ecology, Ecography 23 (2000) $101-113$.

[10] M. F. Tognelli, D. A. Kelt, Analysis of determinants of mammalian species richness in South America using spatial autoregressive models, Ecography 27(4) (2004) 427-436.

[11] J. LeSage, An introduction to spatial econometrics, Revue d'économie industrielle 123 (2008) 19-44.

[12] J. Elhorst, Applied spatial econometrics: Raising the bar, Spatial Economic Analysis 5(1) (2010) 9-28.

[13] W. Conover, Practical Nonparametric Statistics, John Wiley \& Sons, Inc., 3rd edition, 1999.

[14] N. Salkind, Encyclopedia of Research Design, SAGE Publications, Inc., 2010.

[15] L. Guo, Z. Ma, L. Zhang, Comparison of bandwidth selection in application of geographically weighted regression: a case study, Canadian Journal of Forest Research 38 (2008) 2526-2534.

[16] L. Zhang, Z. Ma, L. Guo, An evaluation of spatial autocorrelation and heterogeneity in the residuals of six regression models, Forest Science 55(6) (2009) 533-548.

[17] R. J. Davis, A. Keeley, Feathering of footwear, Science and Justice 40(4) (2000) 273-276. 
[18] SWGTREAD, Range of conclusions for footwear and tire impression examinations, (https://www.nist.gov/sites/default/files/documents/2016/10/26/swgtread_ 10_range_of_conclusions_standard_for_footwear_and_tire_impression_ examinations_201303.pdf), 2013.

[19] European Expert Group on Fingerprint Identification II, Method for Fingerprint Identification Part II: Detailing the Method Using Common Terminology and through the Definition and Application of Shared Principles, Technical Report, INTERPOL, 2004.

[20] Y. Chen, A. Jain, Beyond minutiae: A fingerprint individulity model with pattern, ridge and pore features, in: International Conference on Biometrics: Advances in Biometrics, pp. 523-533. 


\section{Light Wear}

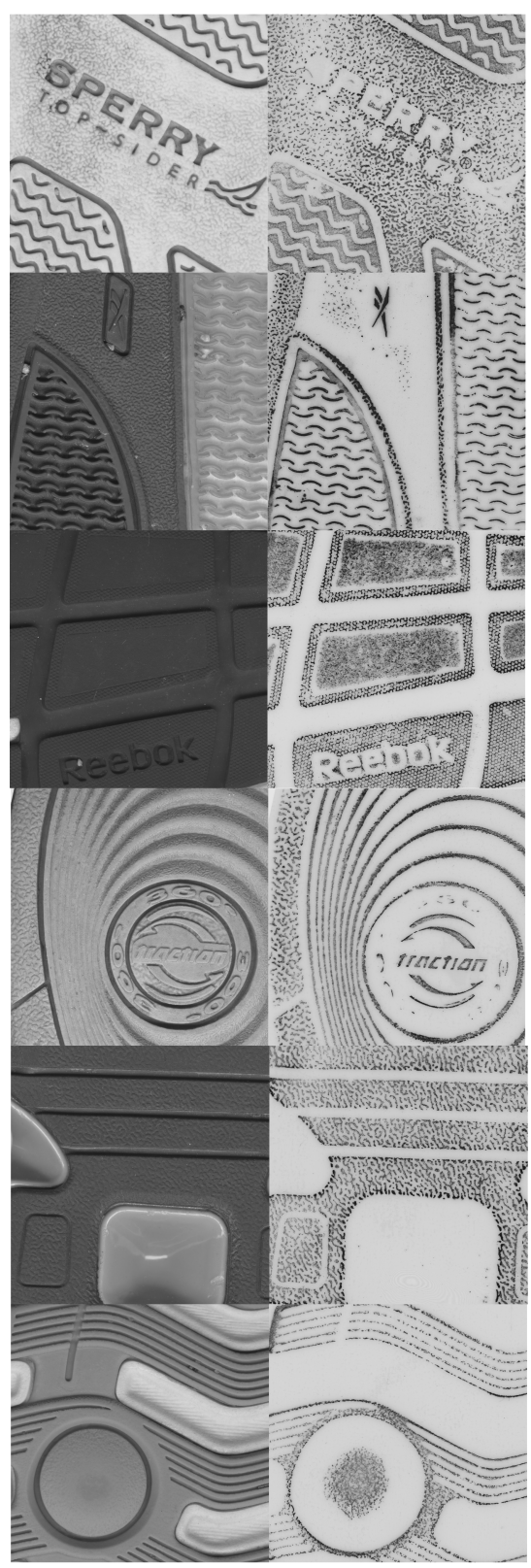

Moderate Wear
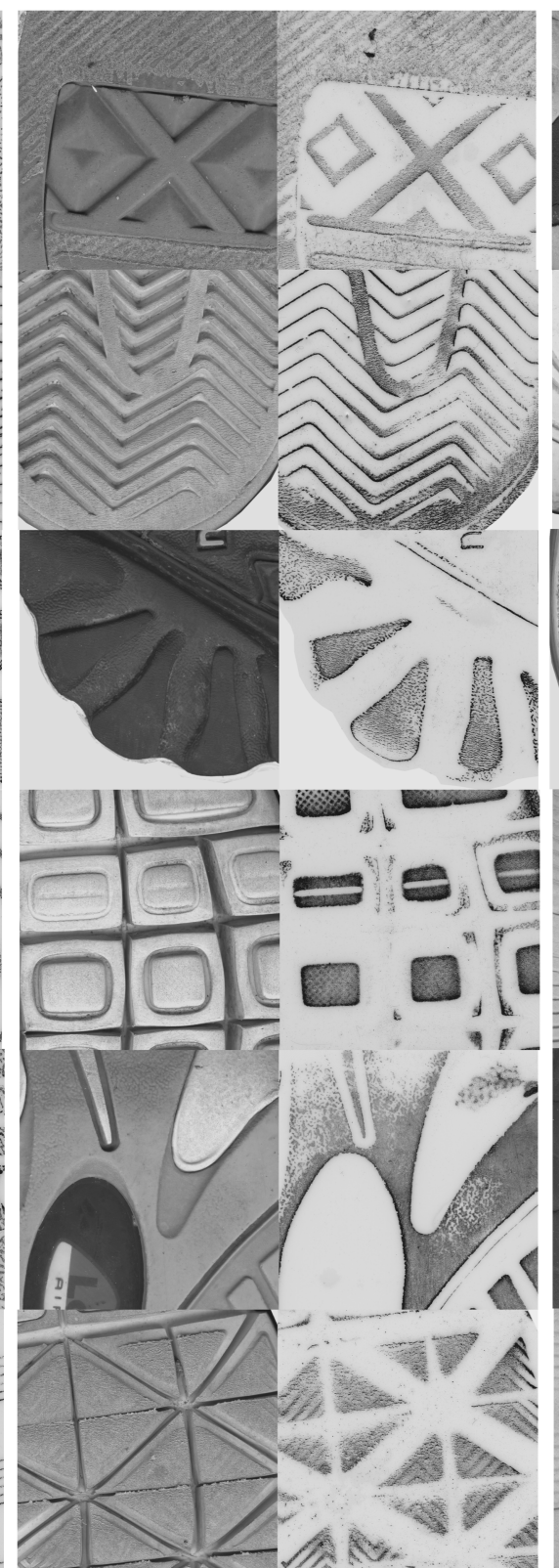

High Wear
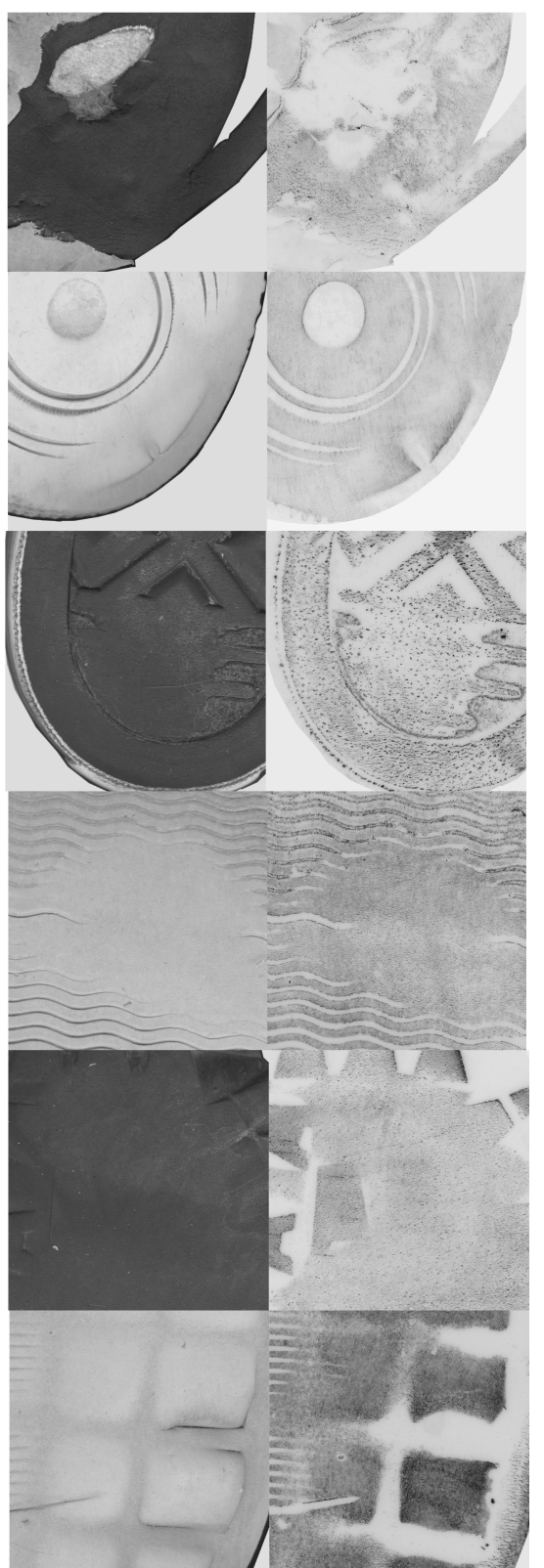

Figure A.1: Examples of light, moderate, and high degrees of wear on localized shoe regions. For each image pair, the left image is the outsole and the right image is the Handiprint exemplar impression. 

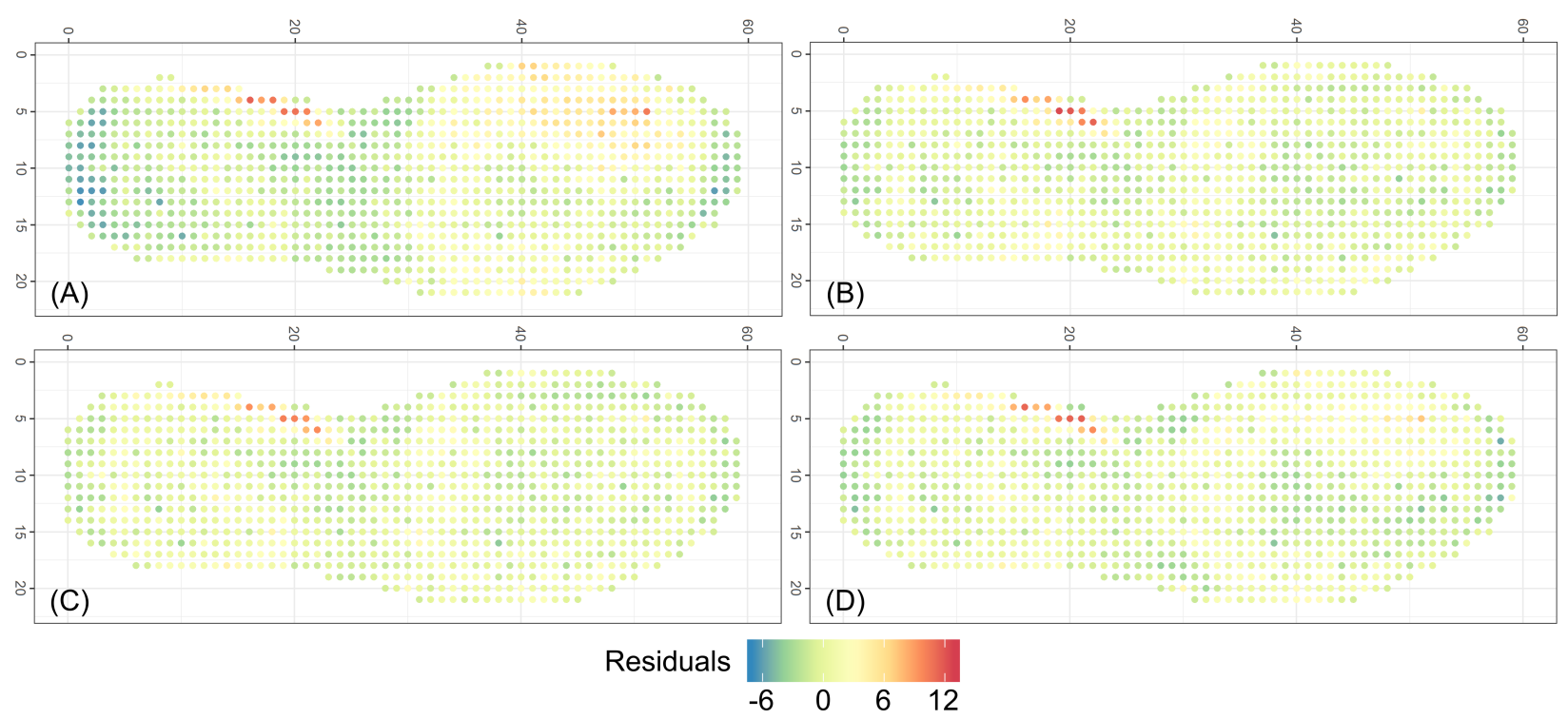

Figure A.2: Pearson residuals (color) of RAC counts as a function of contact area (with a bin percentage offset) obtained from four different models and the full database of 1,300 shoes: (A) non-spatial Poisson GLM, (B) spatial auto-Poisson, (C) spatial Durbin, (D) geographically weighted Poisson regression. For all spatial models, a neighborhood cutoff value of $800 \mathrm{px}$ (approximately $34 \mathrm{~mm}$ ) was used.
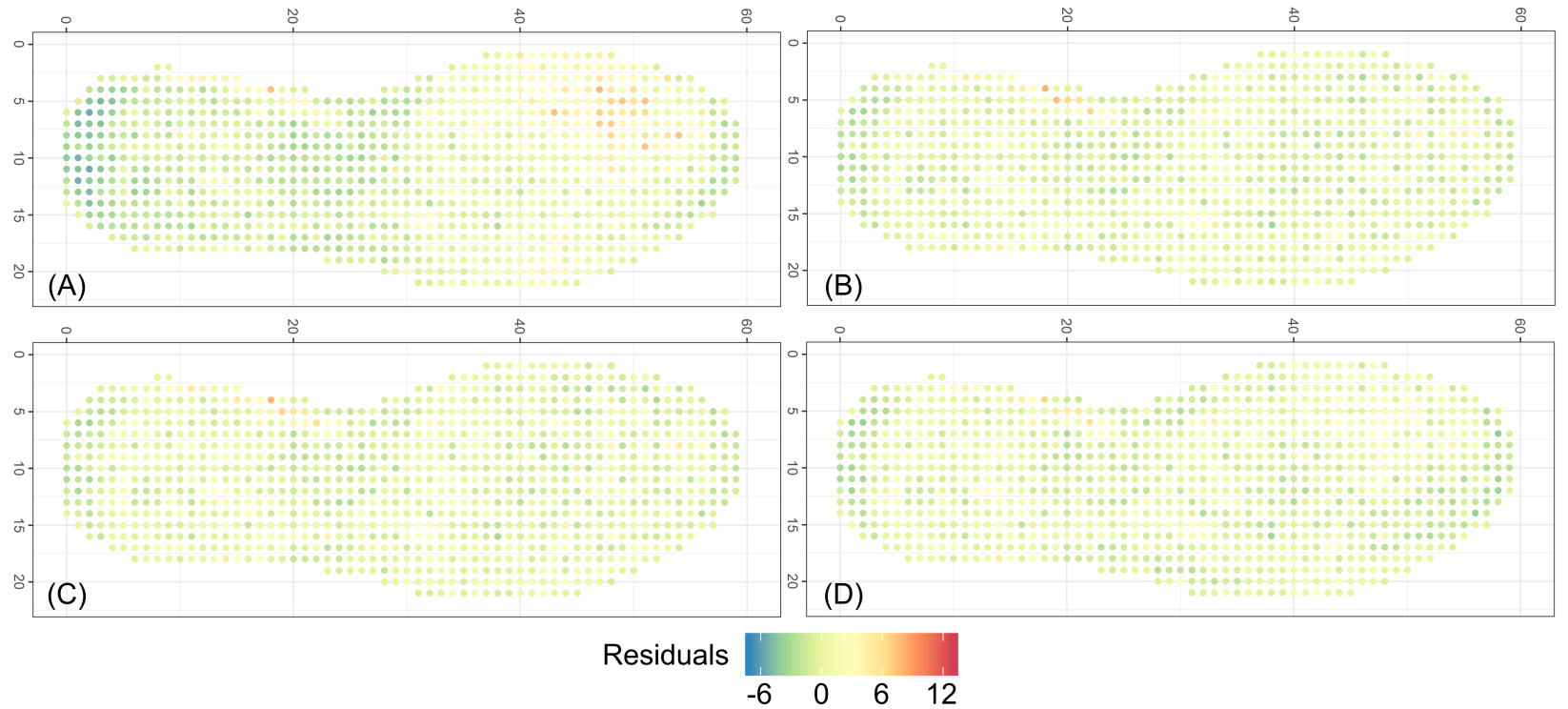

Figure A.3: Pearson residuals (color) of RAC counts as a function of contact area (with a bin percentage offset) obtained from four different models and the subset database of 130 shoes: (A) non-spatial Poisson GLM, (B) spatial auto-Poisson, (C) spatial Durbin, (D) geographically weighted Poisson regression. For all spatial models, a neighborhood cutoff value of 800px (approximately $34 \mathrm{~mm}$ ) was used. 
Table A.1: Summary statistics for model coefficients for RAC counts as a function of contact area (with a bin percentage offset) for the subset database of 130 shoes using geographically weighted Poisson regression (GWPR).

\begin{tabular}{|c|c|c|c|c|c|c|}
\hline \multicolumn{2}{|c|}{ Metric } & Mean & $\mathrm{SD}$ & Median & Minimum & Maximum \\
\hline \multirow{3}{*}{$\begin{array}{l}400 \mathrm{px} / \\
17 \mathrm{~mm}\end{array}$} & $\begin{array}{c}\text { Intercept } \\
\left(\beta_{0}\right)\end{array}$ & 2.598 & 0.8778 & 2.479 & 1.154 & 4.272 \\
\hline & $\begin{array}{c}\text { Contact } \\
\left(\beta_{1}\right)\end{array}$ & $1.4 \mathrm{E}-04$ & $1.2 \mathrm{E}-04$ & $1.4 \mathrm{E}-04$ & $-7.2 \mathrm{E}-05$ & $3.7 \mathrm{E}-04$ \\
\hline & $\begin{array}{c}\log (\operatorname{Bin}) \\
\left(\beta_{2}\right)\end{array}$ & 1.085 & 0.7351 & 0.8511 & -0.1250 & 3.338 \\
\hline \multirow{3}{*}{$\begin{array}{l}800 \mathrm{px} / \\
34 \mathrm{~mm}\end{array}$} & $\begin{array}{c}\text { Intercept } \\
\left(\beta_{0}\right)\end{array}$ & 2.480 & 0.7425 & 2.418 & 1.469 & 3.977 \\
\hline & $\begin{array}{c}\text { Contact } \\
\left(\beta_{1}\right)\end{array}$ & $1.5 \mathrm{E}-04$ & $1.0 \mathrm{E}-04$ & $1.5 \mathrm{E}-04$ & $-3.8 \mathrm{E}-05$ & $3.2 \mathrm{E}-04$ \\
\hline & $\begin{array}{c}\log (\operatorname{Bin}) \\
\left(\beta_{2}\right) \\
\end{array}$ & 0.9429 & 0.5805 & 0.6920 & 0.2148 & 2.566 \\
\hline \multirow{3}{*}{$\begin{array}{c}1200 \mathrm{px} / \\
51 \mathrm{~mm}\end{array}$} & $\begin{array}{c}\text { Intercept } \\
\left(\beta_{0}\right)\end{array}$ & 2.296 & 0.5180 & 2.200 & 1.672 & 3.649 \\
\hline & $\begin{array}{c}\text { Contact } \\
\left(\beta_{1}\right)\end{array}$ & $1.8 \mathrm{E}-04$ & $7.5 \mathrm{E}-05$ & $1.8 \mathrm{E}-04$ & $1.2 \mathrm{E}-05$ & $2.9 \mathrm{E}-04$ \\
\hline & $\begin{array}{c}\log (\operatorname{Bin}) \\
\left(\beta_{2}\right)\end{array}$ & 0.8265 & 0.3903 & 0.6844 & 0.3571 & 1.972 \\
\hline
\end{tabular}


Table A.2: Summary statistics for model coefficients for RAC counts as a function of contact-localized wear (with a bin percentage offset) for the subset database of 130 shoes using geographically weighted Poisson regression (GWPR).

\begin{tabular}{|c|c|c|c|c|c|c|}
\hline \multicolumn{2}{|c|}{ Metric } & Mean & $\mathrm{SD}$ & Median & Minimum & Maximum \\
\hline \multirow{5}{*}{$\begin{array}{l}400 \mathrm{px} / \\
17 \mathrm{~mm}\end{array}$} & $\begin{array}{c}\text { Intercept } \\
\left(\beta_{0}\right)\end{array}$ & 2.602 & 0.8865 & 2.521 & 0.1206 & 4.274 \\
\hline & $\begin{array}{c}\text { Light } \\
\left(\beta_{1}\right)\end{array}$ & $-8.1 \mathrm{E}-05$ & $2.6 \mathrm{E}-04$ & $-6.6 \mathrm{E}-05$ & $-7.0 \mathrm{E}-04$ & $4.8 \mathrm{E}-04$ \\
\hline & $\begin{array}{c}\text { Moderate } \\
\left(\beta_{2}\right)\end{array}$ & $1.9 \mathrm{E}-04$ & $1.0 \mathrm{E}-04$ & $1.9 \mathrm{E}-04$ & $-2.0 \mathrm{E}-05$ & 5.1E-04 \\
\hline & $\begin{array}{l}\text { High } \\
\left(\beta_{3}\right)\end{array}$ & $1.3 \mathrm{E}-05$ & $2.6 \mathrm{E}-04$ & $-3.0 \mathrm{E}-05$ & $-6.0 \mathrm{E}-04$ & $9.7 \mathrm{E}-04$ \\
\hline & $\begin{array}{c}\log (\operatorname{Bin}) \\
\left(\beta_{4}\right)\end{array}$ & 1.134 & 0.5984 & 0.9572 & 0.1169 & 3.070 \\
\hline \multirow{5}{*}{$\begin{array}{l}800 \mathrm{px} / \\
34 \mathrm{~mm}\end{array}$} & $\begin{array}{c}\text { Intercept } \\
\left(\beta_{0}\right)\end{array}$ & 2.510 & 0.7499 & 2.321 & 1.559 & 3.940 \\
\hline & $\begin{array}{l}\text { Light } \\
\left(\beta_{1}\right)\end{array}$ & $-7.3 \mathrm{E}-05$ & $2.3 \mathrm{E}-04$ & $-6.8 \mathrm{E}-05$ & $-4.3 \mathrm{E}-04$ & $3.0 \mathrm{E}-04$ \\
\hline & $\begin{array}{c}\text { Moderate } \\
\left(\beta_{2}\right)\end{array}$ & $2.0 \mathrm{E}-04$ & $7.7 \mathrm{E}-05$ & $2.1 \mathrm{E}-04$ & $4.6 \mathrm{E}-05$ & $3.2 \mathrm{E}-04$ \\
\hline & $\begin{array}{l}\text { High } \\
\left(\beta_{3}\right)\end{array}$ & $4.8 \mathrm{E}-06$ & $1.3 \mathrm{E}-04$ & $-1.9 \mathrm{E}-05$ & $-2.8 \mathrm{E}-04$ & $3.1 \mathrm{E}-04$ \\
\hline & $\begin{array}{c}\log (\operatorname{Bin}) \\
\left(\beta_{4}\right)\end{array}$ & 1.027 & 0.5701 & 0.8840 & 0.2713 & 2.503 \\
\hline \multirow{5}{*}{$\begin{array}{c}1200 \mathrm{px} / \\
51 \mathrm{~mm}\end{array}$} & $\begin{array}{c}\text { Intercept } \\
\left(\beta_{0}\right)\end{array}$ & 2.422 & 0.5591 & 1.967 & 1.824 & 3.702 \\
\hline & $\begin{array}{l}\text { Light } \\
\left(\beta_{1}\right)\end{array}$ & $-8.9 \mathrm{E}-05$ & $1.6 \mathrm{E}-04$ & $-4.5 \mathrm{E}-05$ & $-4.2 \mathrm{E}-04$ & $3.0 \mathrm{E}-04$ \\
\hline & $\begin{array}{c}\text { Moderate } \\
\left(\beta_{2}\right)\end{array}$ & $2.3 \mathrm{E}-04$ & $5.8 \mathrm{E}-05$ & $2.4 \mathrm{E}-04$ & $8.6 \mathrm{E}-05$ & $3.0 \mathrm{E}-04$ \\
\hline & $\begin{array}{l}\text { High } \\
\left(\beta_{3}\right)\end{array}$ & $-9.1 \mathrm{E}-06$ & $7.6 \mathrm{E}-05$ & $-2.1 \mathrm{E}-05$ & $-1.7 \mathrm{E}-04$ & $1.3 \mathrm{E}-04$ \\
\hline & $\begin{array}{c}\log (\operatorname{Bin}) \\
\left(\beta_{4}\right)\end{array}$ & 0.9821 & 0.4165 & 0.8351 & 0.5203 & 2.100 \\
\hline
\end{tabular}



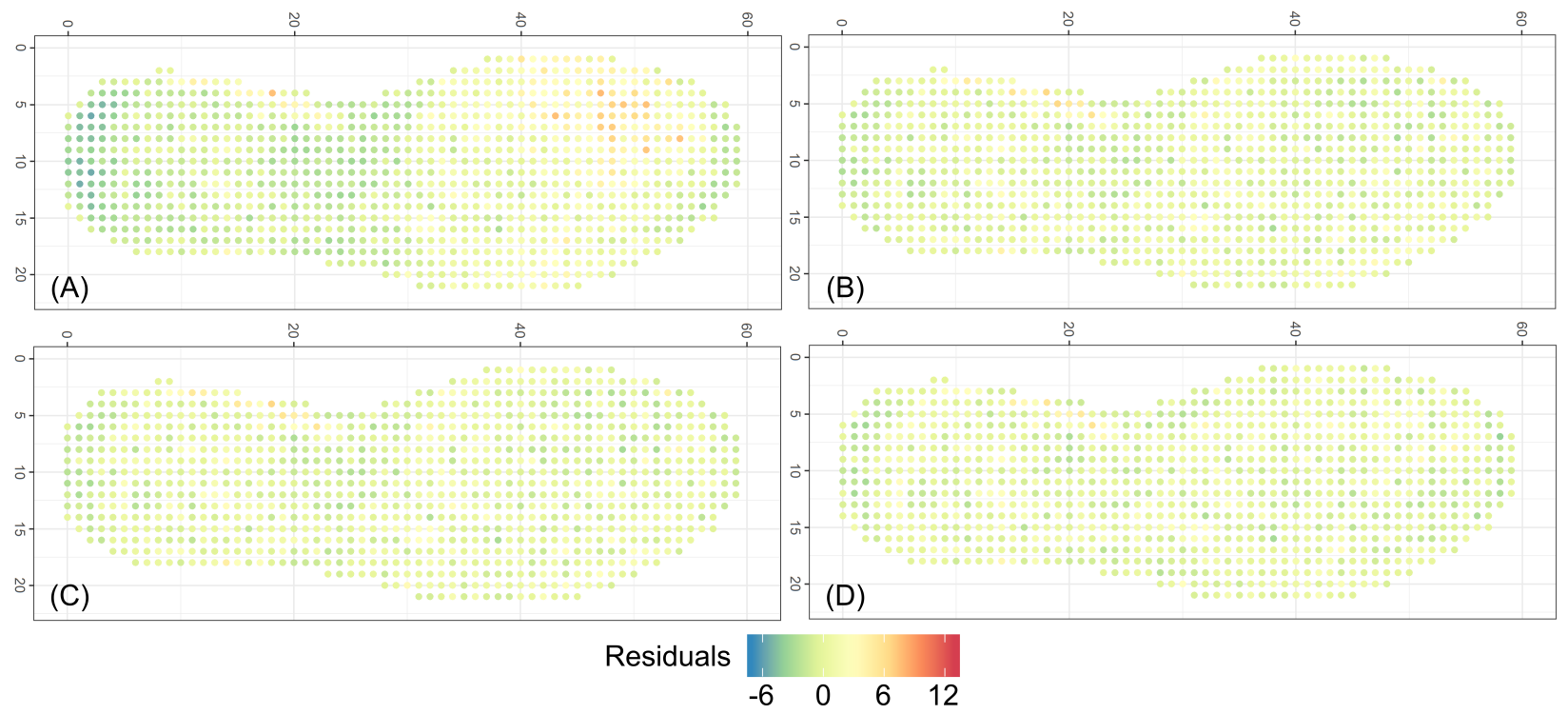

Figure A.4: Pearson residuals (color) of RAC counts as a function of contact area (with a bin percentage offset) obtained from four different models and the subset database of 130 shoes: (A) non-spatial Poisson GLM, (B) spatial auto-Poisson, (C) spatial Durbin, (D) geographically weighted Poisson regression. For all spatial models, a neighborhood cutoff value of 400px (approximately $17 \mathrm{~mm}$ ) was used.
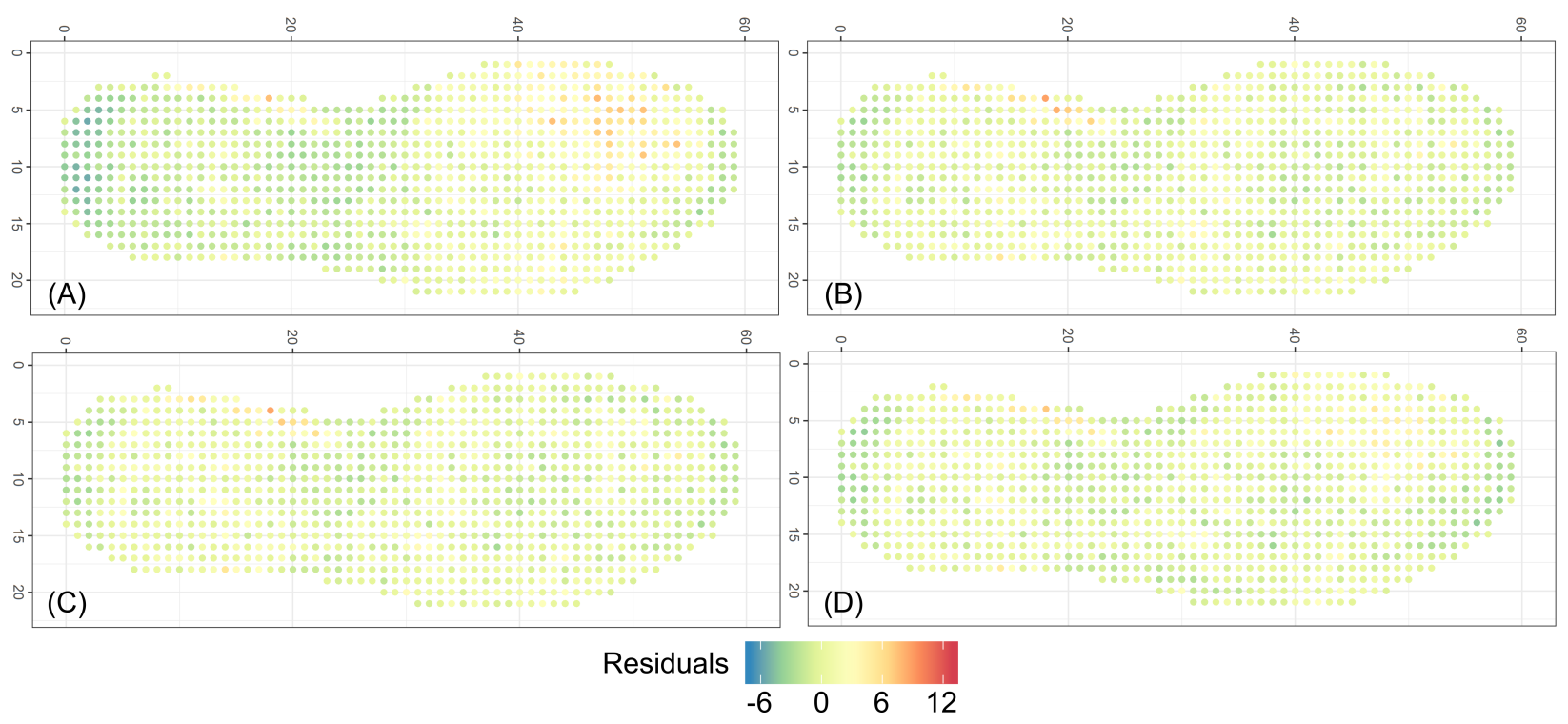

Figure A.5: Pearson residuals (color) of RAC counts as a function of contact area (with a bin percentage offset) obtained from four different models and the subset database of 130 shoes: (A) non-spatial Poisson GLM, (B) spatial auto-Poisson, (C) spatial Durbin, (D) geographically weighted Poisson regression. For all spatial models, a neighborhood cutoff value of 1200px (approximately $51 \mathrm{~mm}$ ) was used. 

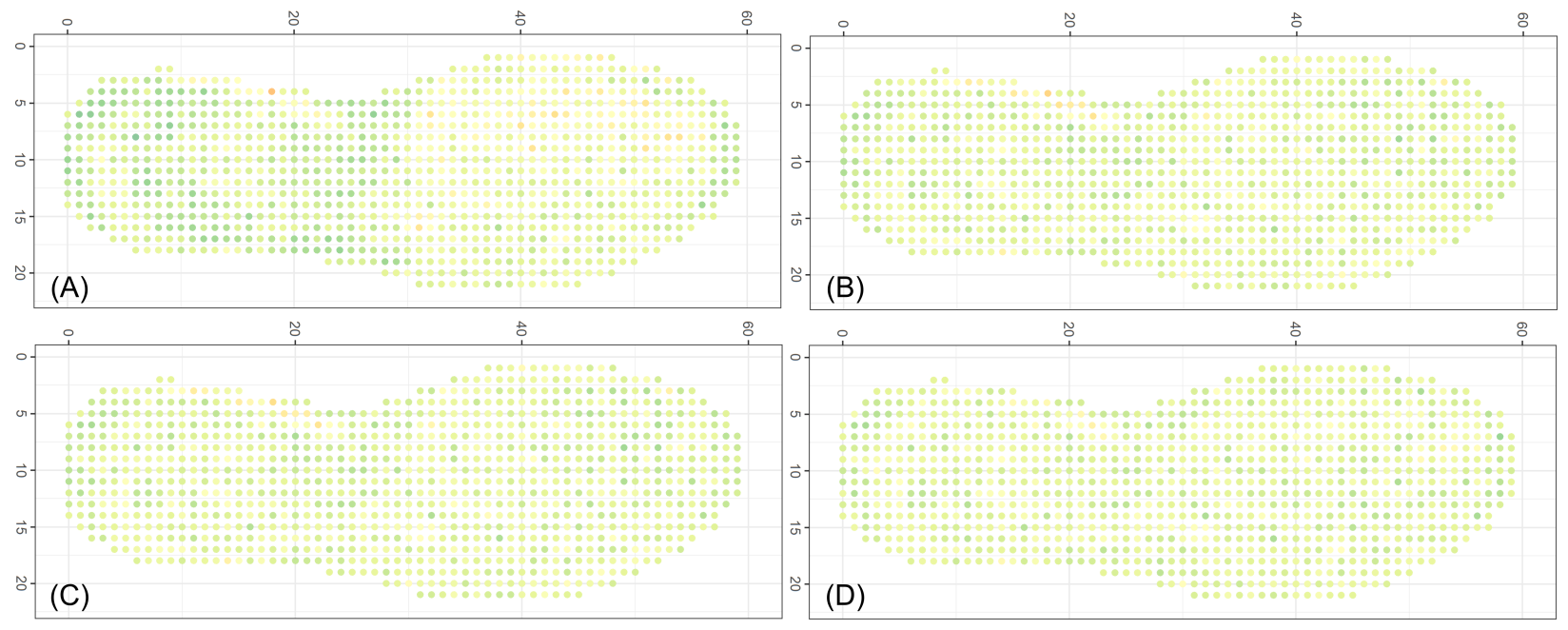

Residuals

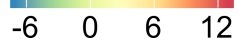

Figure A.6: Pearson residuals (color) of RAC counts as a function of contact-localized wear (with a bin percentage offset) obtained from four different models and the subset database of 130 shoes: (A) non-spatial Poisson GLM, (B) spatial auto-Poisson, (C) spatial Durbin, (D) geographically weighted Poisson regression. For all spatial models, a neighborhood cutoff value of 400px (approximately $17 \mathrm{~mm}$ ) was used.
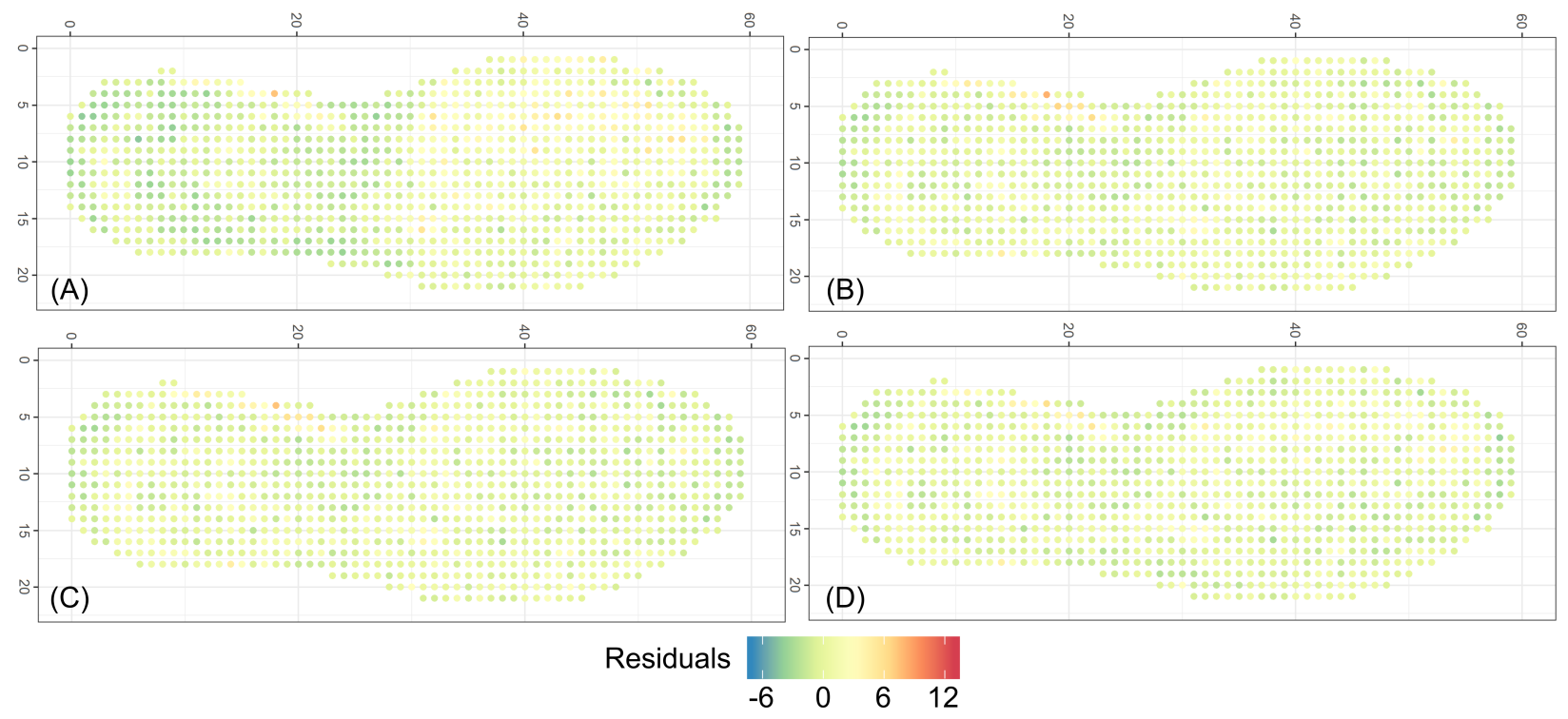

Figure A.7: Pearson residuals (color) of RAC counts as a function of contact-localized wear (with a bin percentage offset) obtained from four different models and the subset database of 130 shoes: (A) non-spatial Poisson GLM, (B) spatial auto-Poisson, (C) spatial Durbin, (D) geographically weighted Poisson regression. For all spatial models, a neighborhood cutoff value of 800px (approximately $34 \mathrm{~mm}$ ) was used. 

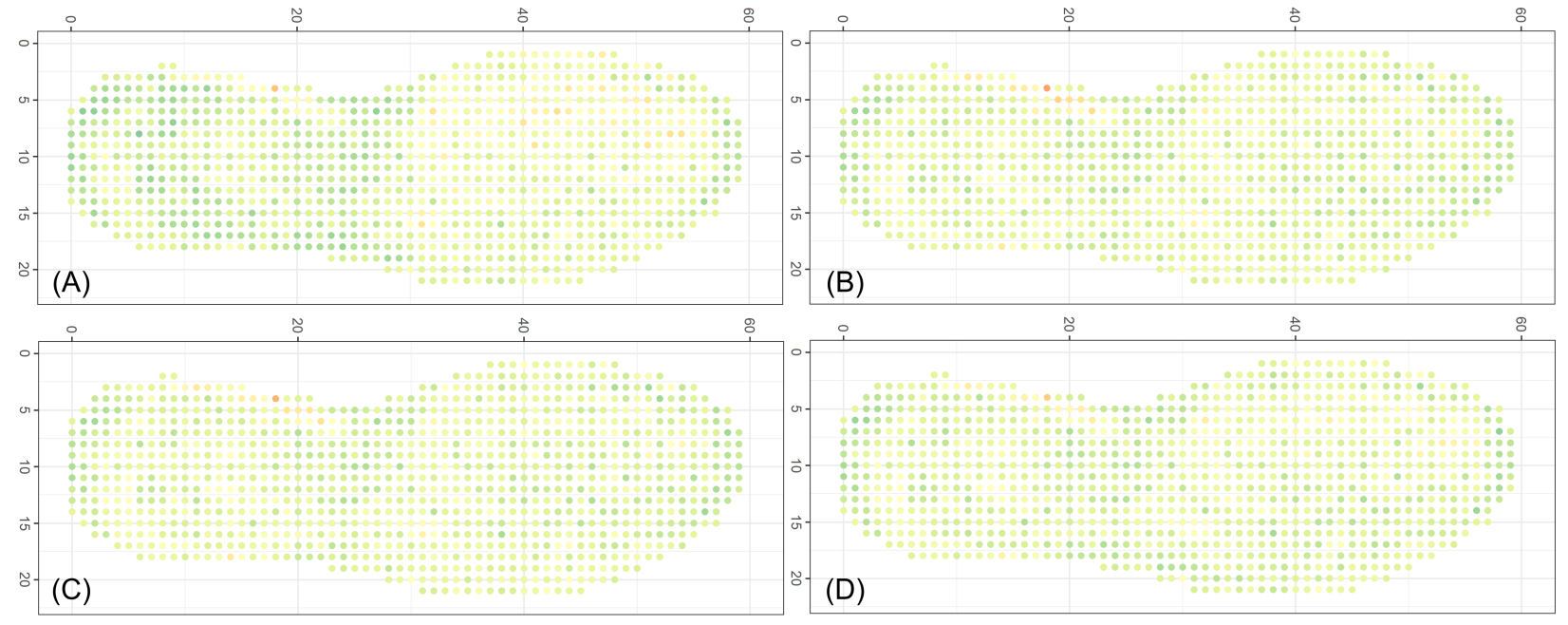

Residuals

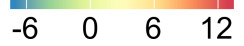

Figure A.8: Pearson residuals (color) of RAC counts as a function of contact-localized wear (with a bin percentage offset) obtained from four different models and the subset database of 130 shoes: (A) non-spatial Poisson GLM, (B) spatial auto-Poisson, (C) spatial Durbin, (D) geographically weighted Poisson regression. For all spatial models, a neighborhood cutoff value of 1200px (approximately 51mm) was used.
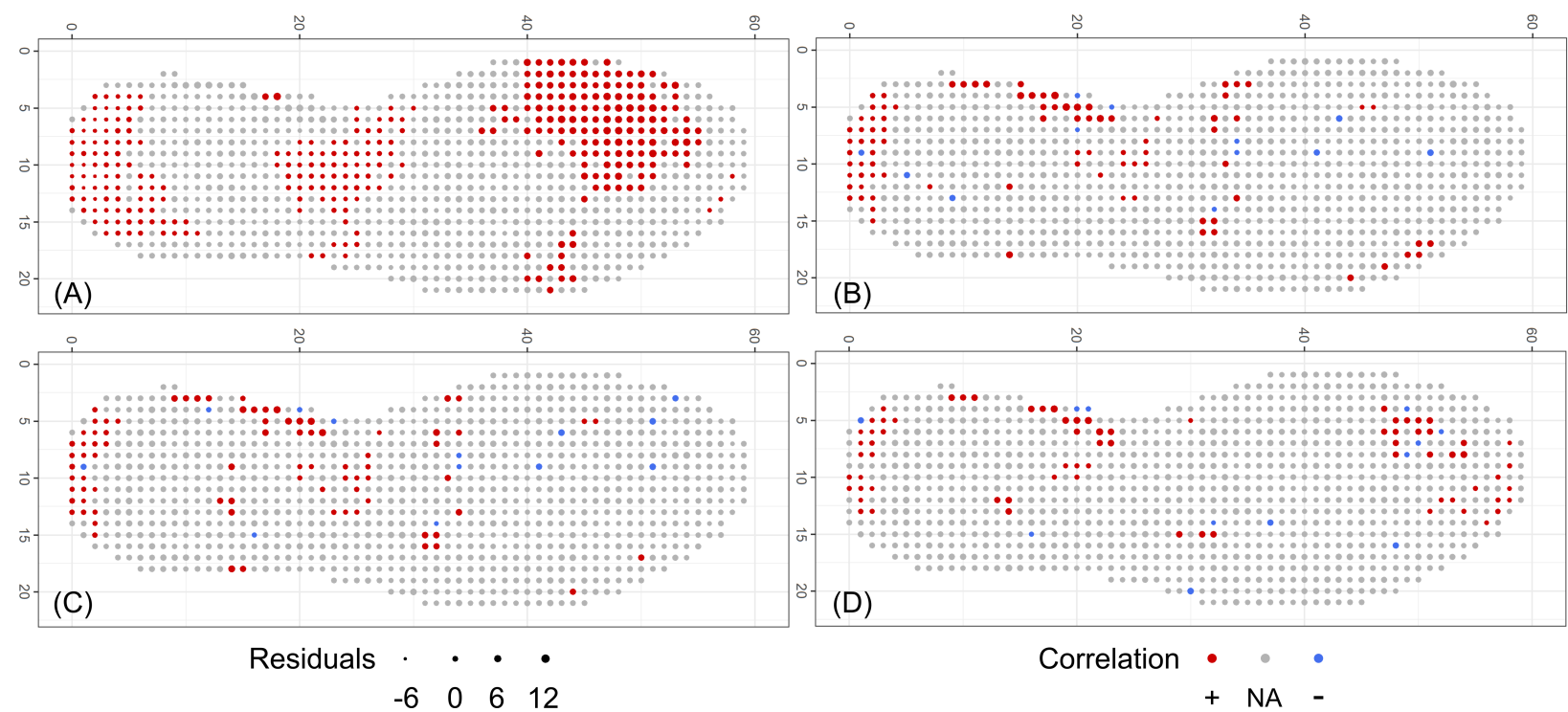

Figure A.9: Spatial correlation detected (red: positive, blue: negative, gray: none) and associated Pearson residuals (size) of RAC counts predicted as a function of contact area (with a bin percentage offset) for the subset database of 130 shoes. For all spatial models, a neighborhood cutoff value of 400px (approximately $17 \mathrm{~mm})$ was used. 

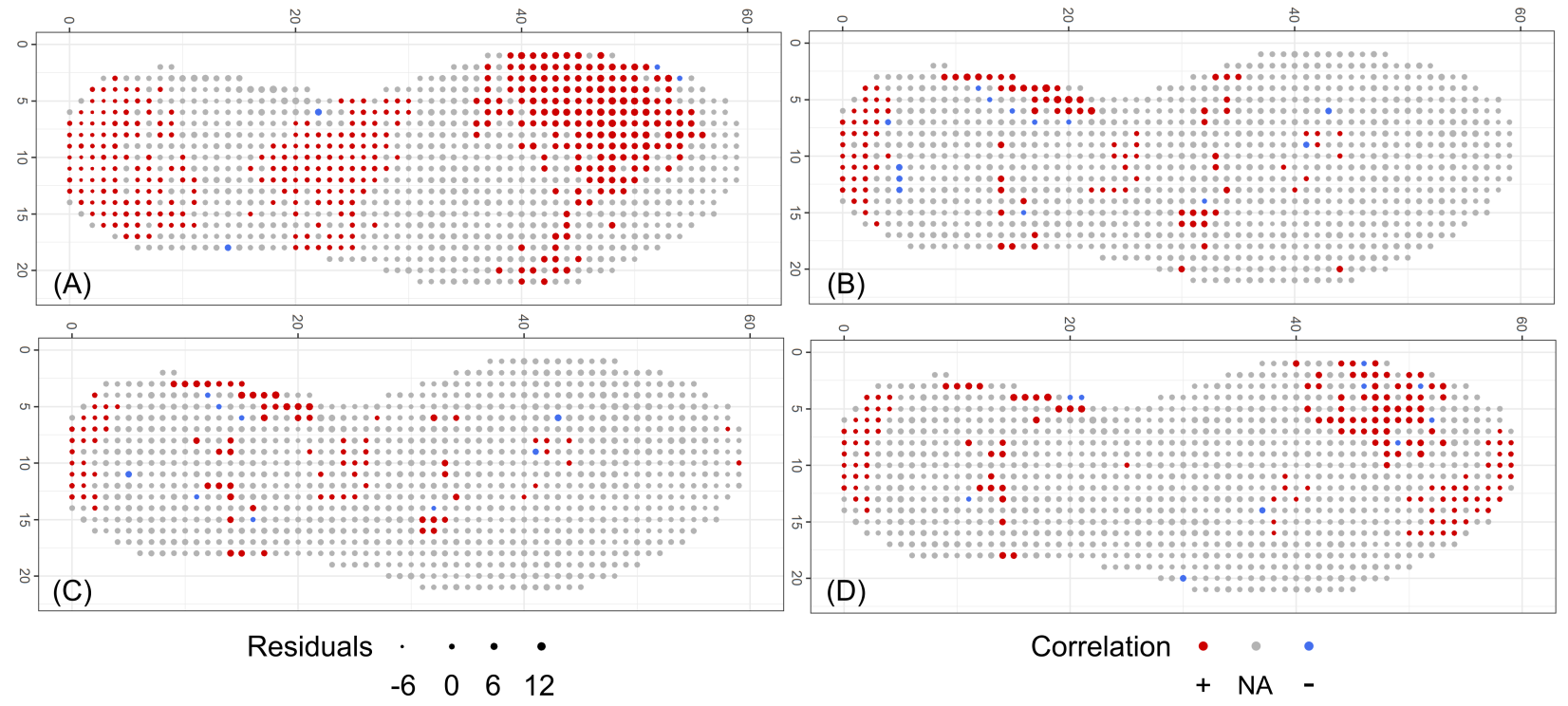

Figure A.10: Spatial correlation detected (red: positive, blue: negative, gray: none) and associated Pearson residuals (size) of RAC counts predicted as a function of contact area (with a bin percentage offset) for the subset database of 130 shoes. For all spatial models, a neighborhood cutoff value of 800px (approximately $34 \mathrm{~mm})$ was used.
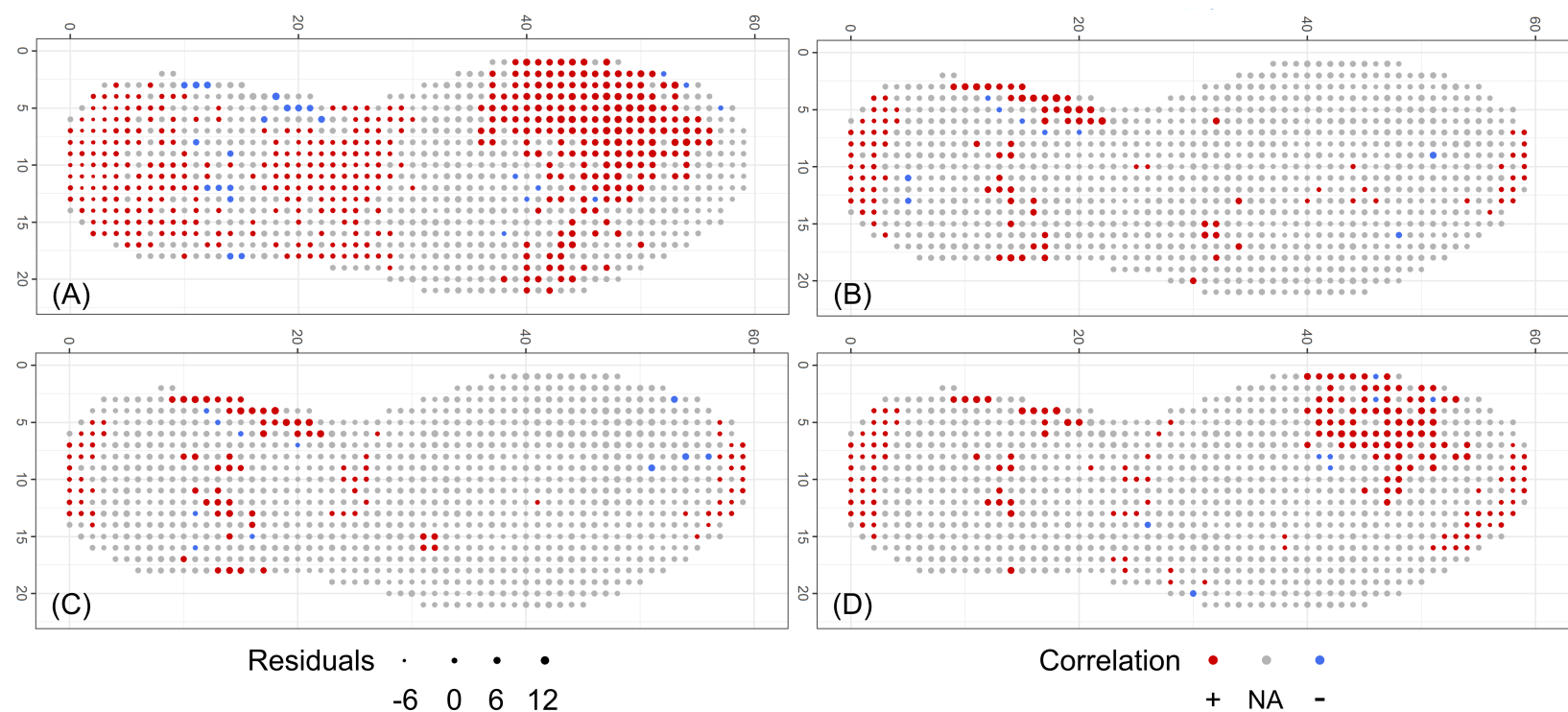

Figure A.11: Spatial correlation detected (red: positive, blue: negative, gray: none) and associated Pearson residuals (size) of RAC counts predicted as a function of contact area (with a bin percentage offset) for the subset database of 130 shoes. For all spatial models, a neighborhood cutoff value of 1,200px (approximately $51 \mathrm{~mm})$ was used. 

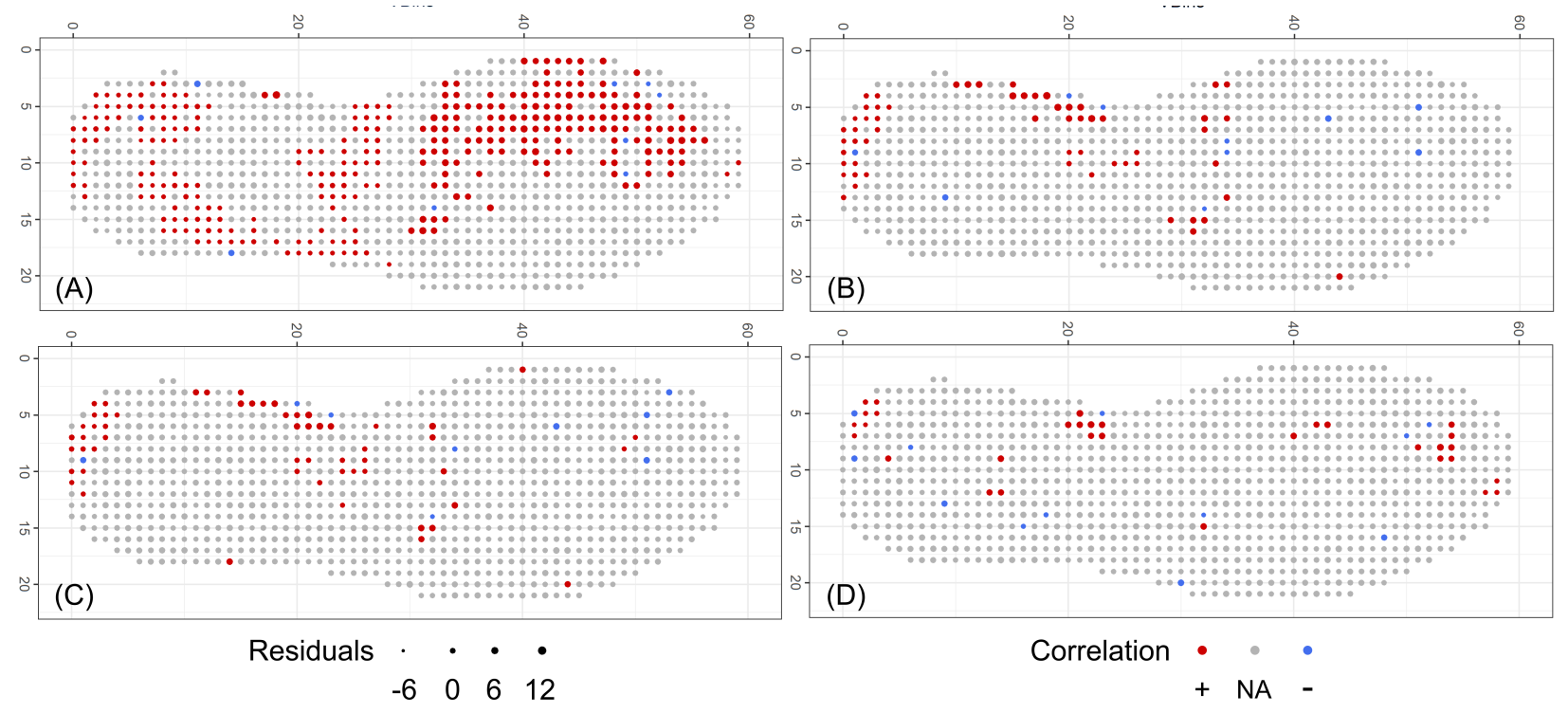

Figure A.12: Spatial correlation detected (red: positive, blue: negative, gray: none) and associated Pearson residuals (size) of RAC counts predicted as a function of contact-localized wear (with a bin percentage offset) for the subset database of 130 shoes. For all spatial models, a neighborhood cutoff value of 400px (approximately $17 \mathrm{~mm}$ ) was used.
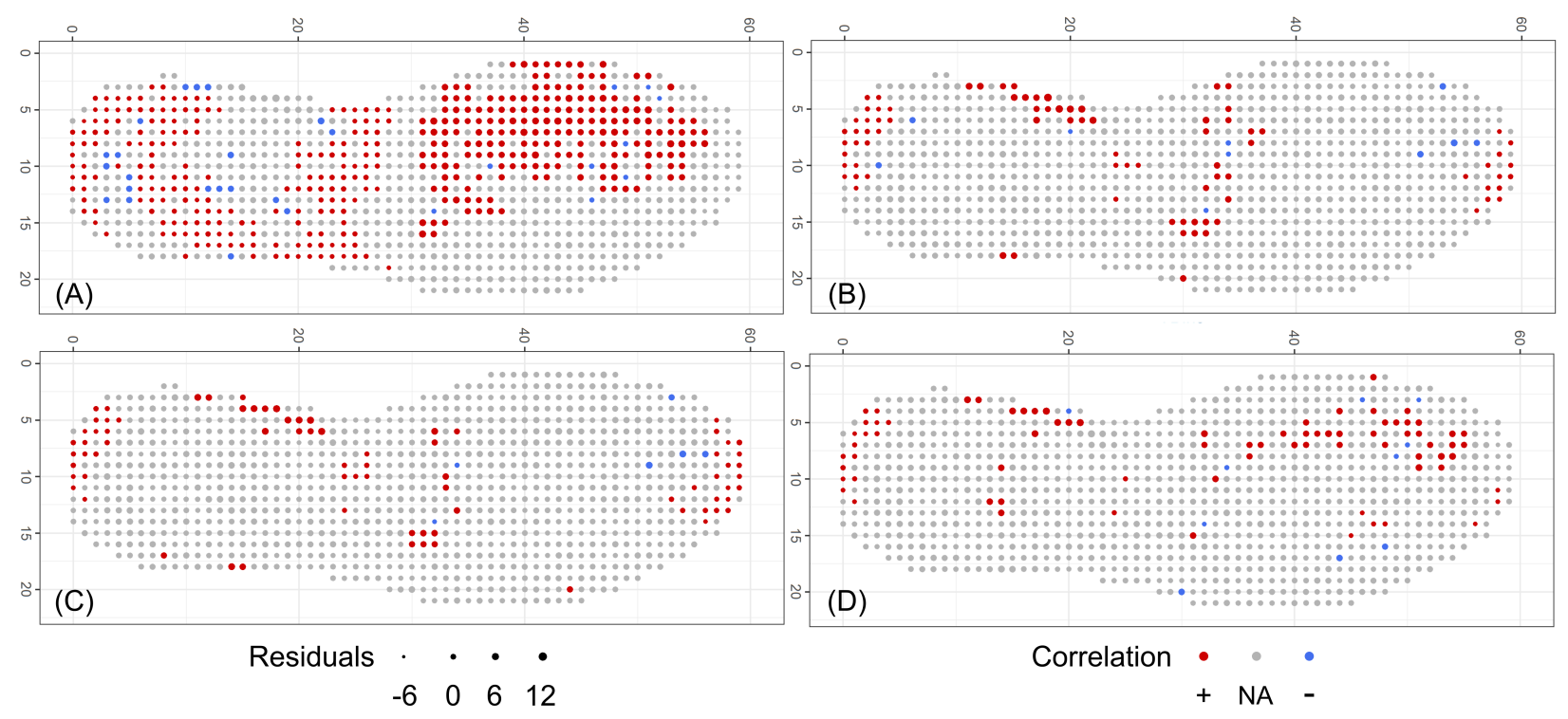

Figure A.13: Spatial correlation detected (red: positive, blue: negative, gray: none) and associated Pearson residuals (size) of RAC counts predicted as a function of contact-localized wear (with a bin percentage offset) for the subset database of 130 shoes. For all spatial models, a neighborhood cutoff value of 800px (approximately $34 \mathrm{~mm}$ ) was used. 

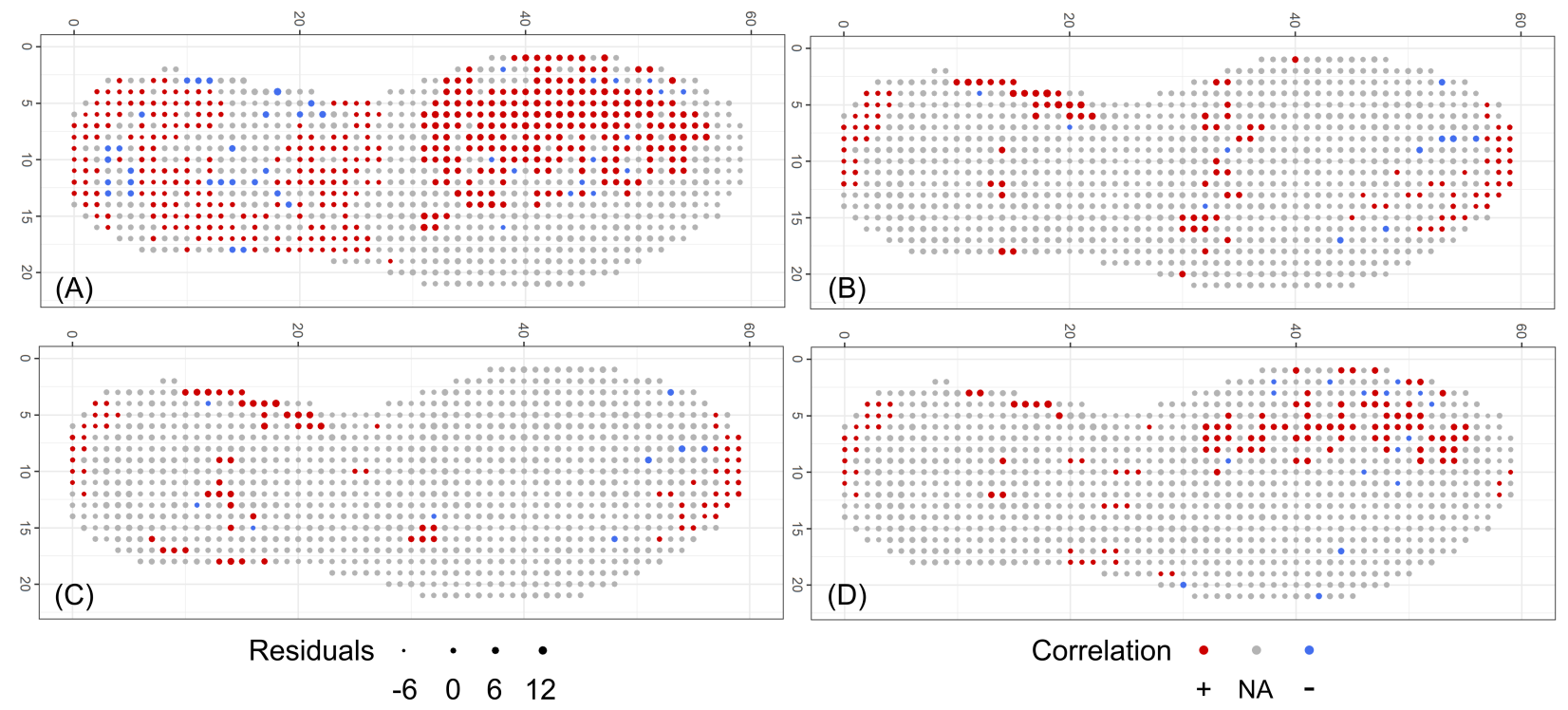

Figure A.14: Spatial correlation detected (red: positive, blue: negative, gray: none) and associated Pearson residuals (size) of RAC counts predicted as a function of contact-localized (with a bin percentage offset) for the subset database of 130 shoes. For all spatial models, a neighborhood cutoff value of 1,200px (approximately $51 \mathrm{~mm}$ ) was used. 


\section{Final Remarks}

The primary objective of this research was to characterize two factors (chance association and spatial distributions) that underpin weight of evidence assignments for randomly acquired characteristics.

\subsection{Observations and Future Directions}

\subsubsection{Chance Association of RACs}

Using a large-scale database of 72,306 accidentals, identified on 1,300 outsoles, chance association was ascertained through a combination of empirical computation, visual observation, and statistical modeling across 987 locations on a standardized heatmap outsole. Results indicate that conservative estimates for median chance associations (based upon the upper 95\% credible interval) ranged from 1 in 291,111 for compact features to 1 in 880,774 for variable features, with linear features exhibiting an estimated probability of 1 in 444,126. These probabilities were computed as the intersection of positional co-occurrence and visual indistiguishability within a $5 \mathrm{~mm} \times 5 \mathrm{~mm}$ cell. Specifically considering indistinguishability, this value consisted of a combination of empirical and theoretical estimates. Namely, 91,607 visual comparisons were performed in duplicate to determine the number of confused features. These observations were then used to build a logistic regression model that was utilized to predict the probability of confusion for all remaining RAC pairs, as a function of feature similarity. In doing so, the differentiability of RACs was limited to area of overlap, thus constituting a relatively low-level model. Additional research effort is currently being 
invested to incorporate additional explanatory factors, including feature complexity, which may expand or improve upon the existing model.

In addition, although the resulting probabilities of stochastic association indicate that individual RACs are moderately to highly discriminating, these values represent a scenario involving two randomly selected and unrelated shoes from a given collection. While this question addresses the baseline discriminating potential of accidentals and their utility for comparison, random match probability is generally the question of greater interest for source attribution determinations and reporting. In other words, the relevant probability for the trier of fact becomes the chance that an observed association would exist between a questioned impression and any other non-suspect shoe. In an effort to provide further support to the foundational validity of footwear evidence, additional research is being conducted to quantify this random match probability using the same large-scale database of RACs.

\subsubsection{Spatial Distribution of RACs}

As part of this research, the spatial distribution of accidentals was evaluated with respect to two outsole-related factors that were expected to impact the locations and frequencies of features. First, tread design was incorporated into synthetic RAC realizations, representing Poisson null and clustered and dispersed alternative distributions, used to facilitate evaluation of adherence to spatial randomness. Results indicated that traditional tests of complete spatial randomness were under-powered for the assessment of footwear data, likely owing to the inherent non-uniformity of the study space (i.e., RACs cannot develop on any area within the perimeter of the outsole). However, when feature position is simulated based on a homogeneous Poisson process modified by contact area, synthetic models agreed with empirical data across $64 \%$ of the standardized shoe, on average. Although these results were promising and indicated that the majority of all locations were well predicted by contact alone, specific regions in the ball of the toe, arch, and edge of the heel were not adequately described. Therefore, in an effort to better assess RAC distributions as a function of contactarea, regression modeling was implemented. These techniques allowed for an investigation of 
additional exogenous and endogenous spatial effects that might better inform RAC frequency in the remaining, poorly modeled $36 \%$ of the outsole. Overall, this analysis revealed that incorporation of spatial correlations between both neighboring responses (feature counts) and predictors (average contact) improved prediction performance by $67 \%$ under optimal circumstances, and described RAC counts across $87 \%$ of the outsole.

Given the success associated with informing frequency as a function of contact area, it was then hypothesized that degree of wear may be able to further improve predictions for the areas still exhibiting residual correlation. Thus, a proof of concept study was conducted to assess the impact of contact-localized wear, an intersection of contact and use, as a predictor for RAC counts in the regression models. Compared to contact area, which described approximately $91 \%$ of outsole locations in the proof of concept database, contact-localized wear described nearly $96 \%$ of the standardized shoe. Based upon these promising results, this work should be expanded to include the entire database of 1,300 outsoles to verify that observations and conclusions are maintained when including more variation in tread design, regions of wear, and number of identified features.

Likewise, additional research effort could be expended in an effort to further minimize residual spatial association, and several potential areas of inquiry could be investigated. First, the descriptions of wear used in the proof of concept study were reduced to three categories (light, moderate, and high). While this process undoubtedly improved consistency in classification, it likewise removed potentially important nuances (e.g., high wear region that resulted in a hole versus a high wear region that caused complete balding). It is recommended that future work consider inclusion of sub-categories to account for some of these inherent variations, but that could also be easily collapsed into this three-level system to allow for inter-comparison. In addition, alternative methods for describing local regions of wear can be explored. Given the interest in more objective/automated methods in pattern disciplines of forensic science, image processing techniques for quantitatively assigning degrees of wear, such as texture metrics or longitudinal wear-studies to characterize wear appearance, may be worth investigation. Lastly, future work may benefit from model expansion, to include additional predictors (such as outsole material or the presence of Schallamach patterns) as well 
as additional neighborhood sizes. It should be acknowledged, however, that any performance improvements as a result of the aforementioned work will likely be relatively incremental, considering that more than $90 \%$ (and as high as 95.5\%) of outsole locations are adequately predicted using the current models.

Additionally, although the empirical RAC distributions were reliably characterized by the spatial regression modeling techniques utilized in this work, these results need to be expanded in order to be practically incorporated into weight of evidence assessments downstream. In other words, the frequency of features as a function of position is only one part of RAC rarity, which ultimately underpins the value of an observed association. The results of this study indicated that RAC counts varied across space, and hence did not exhibit a uniform point intensity. Thus, the first extension of this work should update modeling of accidental counts to modeling of a 2-dimensional probability density function, wherein the value at each location becomes the probability of encountering a feature given the underlying contactlocalized degree of wear and any necessary spatial correlations (depending on neighborhood size of interest). Subsequently, additional factors should be incorporated into these models and probability estimates, including feature geometry (shape, size, orientation, etc.) and even propensity for visual indistinguishability, in order to examine if/how these vary across space as well. In other words, it is necessary to determine whether each of these factors likewise exhibits spatial variation, similar to what was observed for RAC counts, in order to properly inform evidentiary value.

Furthermore, it should be acknowledged that these types of studies/observations are useful for both qualitative (current practice) and quantitative (future consideration) assessments of weight of evidence. However, in accordance with the desire for forensic pattern disciplines to incorporate more quantitative and objective metrics as part of their examinations, the National Institute of Standards and Technology (NIST) has commenced a project to develop an "end-to-end" system for the analysis of footwear evidence [21]. The major long-term goal of this project is to develop a semi-automated approach that allows for simultaneous consideration of the quantitative weight of various observations made throughout an examination. Therefore, in order to properly inform each phase of the analysis procedure within the system 
(and as conducted by examiners), additional research is required.

\subsection{Addressing Remaining Research Needs}

While this study specifically evaluated two of the major factors that inform weight of evidence determinations of accidentals, thereby addressing fundamental research needs supporting foundational validity, there still remain additional areas of necessary inquiry that must be acknowledged. Moreover, additional features, other than just RACs, must also be incorporated in order to properly ascribe evidentiary value for forensic footwear comparisons. Accordingly, potential topics of research investigation that would allow for a more comprehensive evaluation of weight of evidence include:

- Estimates of population frequency statistics for class characteristics should be gathered. In order to ascertain the value of a crime scene impression, it is necessary to understand the rarity of the manufacturing features, including make, model, and size. However, determination of such frequencies is extremely complex owing to the lack of manufacturing, distribution, and sales information. Existence of counterfeit outsoles likewise complicates this problem. Furthermore, there is currently no way to track how long shoes remain in the population of relevant sources before being discarded or destroyed.

- Characterization of the discriminating potential of general wear should be conducted. Given that wear is a necessary feature used in comparisons and determination of conclusions according to SWGTREAD [5,22], it is imperative to understand the evidential value of observed similarities or discrepancies in wear. Research should specifically focus on a qualitative and quantitative determination of rarity considering the degree, location, size, and orientation of wear patterns.

- Assessment of the evidental value of Schallamach patterns, and other features that could be considered specific wear, should likewise be undertaken. Currently, the SWGTREAD conclusion scale 22 permits decisions of high degree of association with 
observed similarity in specific wear, and potentially lacking patent randomly acquired characteristics. As such, additional inquiry should be invested to provide empirical support for this decision criteria and assigning proper weight to these associations.

- Investigation of the persistence of features should subsequently be conducted. After determining the value of footwear characteristics used in comparisons, it becomes important to understand how they change with additional use. Moreover, since there is often a lapse in time between the commission of the crime and the recovery of suspect shoes, it is important to determine, and ideally predict, how increased wear impacts the number and appearance of expected class, wear, and randomly acquired features.

- Determination of weight of evidence when considering crime scene impressions should also be pursued. Preliminary research outcomes informing foundational validity of footwear evidence comparisons are generally conducted using exemplar imagery, with some studies including artificial degradations or relatively high-quality crime-scene like prints. While these studies characterize the underlying phenomenology, the results are less directly applicable to casework that would be presented in a trial. To allow for a more reliable estimate of value, similar studies must be conducted using evidence-type impressions to properly incorporate complicating factors such as incomplete transfer and degraded clarity. 


\section{Bibliography}

[1] NAS. Strengthening Forensic Science in the United States: A Path Forward; Committee on Identifying the Needs of the Forensic Sciences Community, National Research Council. (https://www.ncjrs.gov/pdffiles1/nij/grants/228091.pdf); 2009.

[2] President's Council of Advisors on Science and Technology. Report to the President: Forensic Science in Criminal Courts: Ensuring Scientific Validity of Feature-Comparison Methods. (https://obamawhitehouse.archives.gov/ sites/default/files/microsites/ostp/PCAST/pcast_forensic_science_ report_final.pdf); 2016.

[3] Bodziak WJ, Hammer L, Johnson GM, Schenck R. Determining the significance of outsole wear characteristics during the forensic examination of footwear impression Evidence. Journal of Forensic Identification. 2012;62(3):254-278.

[4] Bodziak WJ. Forensic Footwear Evidence. Taylor \& Francis Inc; 2016. Available from: http://www.ebook.de/de/product/26652544/william_j_bodziak_forensics_ palm_coast_florida_usa_bodziak_forensic_footwear_evidence.html.

[5] SWGTREAD. Guide for Minimum Qualifications and Training for a Forensic Footwear and/or Tire Tread Examiner; 2006. https://treadforensics. com/images/swgtread/standards/current/swgtread_07_qualifications_ training_200603.pdf.

[6] Bodziak WJ. Footwear Impression Evidence: Detection, Recovery, and Examination. CRC Press; 2000.

[7] Cassidy MJ. Footwear Identification. Canadian Government Printing Centre; 1980. 
[8] Fruchtenicht T, Herzig W, Blackledge R. The discrimination of two-dimensional military boot impression based wear patterns. Science and Justice. 2002;42:97-104.

[9] Hannigan T, Fleury L, Reilly R, O’Mullane B, de Chazal P. Survey of 1276 shoeprint impressions and development of an automatic shoeprint pattern matching facility. Science and Justice. 2006;46:79-89.

[10] Parent S. The significance of class associations in footwear Comparisons. Impression and Pattern Evidence Symposium. 2010;

[11] Gross S, Jeppesen D, Neumann C. The variability and significance of class characterisitcs in footwear impressions. Journal of Forensic Identification. 2013;63(3):332-351.

[12] Fawcett AS. The role of the footmark examiner. Journal of the Forensic Science Society. 1970;10:227-244.

[13] Cassidy MJ. Footwear Identification. Lightning Powder Company, Inc.; 1995.

[14] Stone R. Footwear examination: Mathematical probabilities of theoretical individual characteristics. Journal of Forensic Identification. 2006;56:577-599.

[15] Adair TW, Lemay J, McDonald A, Shaw R, Tewes R. The Mount Bierstadt Study: An Experiment in Unique Damage Formation in Footwear. Journal of Forensic Identification. 2007;57(2):199-205.

[16] Hamburg C, Banks R. Evaluation of the random nature of acquired marks on footwear outsoles; 2010. Impression and Pattern Evidence Symposium.

[17] Wilson H. Comparison of the individual characteristics in the outsoles of thirtynine pairs of Adidas Supernova Classic shoes. Journal of Forensic Identification. 2012;62(3):194-203.

[18] Yekutieli Y, Shor Y, Wiesner S, Tsach T. Expert Assisting Computerized System for Evaluating the Degree of Certainty in 2D Shoeprints. National Institute of Justice; 2012.

[19] Damary N, Mandel M, Wiesner S, Yekutieli Y, Shor Y, Spiegelman C. Dependence 
amount randomly acquired characteristics on shoeprints and their features. Forensic Science International. 2018;283:173-179.

[20] Davis RJ, DeHaan JD. A survey of men's footwear. Journal of the Forensic Science Society. 1977;17:271-285.

[21] Herman M. Oral Presentation: Footwear Impression Research at NIST; 2018. Forensics @ NIST.

[22] SWGTREAD. Range of Conclusions for Footwear and Tire Impression Examinations; 2013. (https://www.nist.gov/sites/default/files/documents/2016/10/ 26/swgtread_10_range_of_conclusions_standard_for_footwear_and_tire_ impression_examinations_201303.pdf). 